\title{
Development and Performance of Detectors for the Cryogenic Dark Matter Search Experiment with an Increased Sensitivity Based on a Maximum Likelihood Analysis of Beta Contamination
}

\author{
by \\ Donald D. Driscoll, Jr.
}

Submitted in partial fulfillment of the requirements for the degree Doctor of Philosophy

Department of Physics

CASE WESTERN RESERVE UNIVERSITY

May 2004 

The dissertation of Donald D. Driscoll, Jr. is approved.

\author{
Lawrence M. Krauss
}

John E. Ruhl

Heather Morrison

Daniel S. Akerib, Committee Chair

Case Western Reserve University

May 2004 
For Diana, Astra, and Marius, who have sacrificed so much in the many months that it took to complete this thesis. 


\section{Contents}

Dedication $\quad$ iv

List of Tables $\quad$ viii

List of Figures $\quad$ X

Abstract $\quad x x$

1 Introduction 1

Works Cited . . . . . . . . . . . . . . . . . . . . . . 5

\begin{tabular}{lll}
\hline $\mathbf{2}$ & WIMPs & $\mathbf{7}$
\end{tabular}

2.1 Dark Matter and Cosmology . . . . . . . . . . . . . . . . . . . . . . . 7

2.2 Particle Dark Matter . . . . . . . . . . . . . . . . . . . . . . . . 8

2.3 WIMP Dark Matter . . . . . . . . . . . . . . . . . . . . . 10

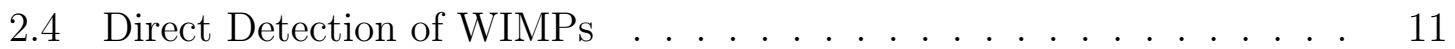

2.5 The CDMS Approach . . . . . . . . . . . . . . . . . . . . . . . . . . . 13

Works Cited . . . . . . . . . . . . . . . . . . . . 17

\begin{tabular}{lll}
\hline 3 & The CDMS Experiment & 19
\end{tabular}

3.1 Cosmic Ray Backgrounds . . . . . . . . . . . . . . . . . . . . . . . . . 19

3.2 Gamma Backgrounds . . . . . . . . . . . . . . . . . . . . . . 20

3.3 Neutron Backgrounds . . . . . . . . . . . . . . . . . . . . . . . . . . . 22

3.4 Beta Backgrounds . . . . . . . . . . . . . . . . . . . . . 23

3.5 Cosmogenic Activation . . . . . . . . . . . . . . . . . . . . . . 23

3.6 Materials and Construction . . . . . . . . . . . . . . . . . . 23

Works Cited . . . . . . . . . . . . . . . . . . . . . . . . 27

4 The ZIP Detector $\quad 29$

4.1 The Physics of an Interaction . . . . . . . . . . . . . . . . . . . . . . 29

4.2 Phonon Channels . . . . . . . . . . . . . . . . . . . . . . . . . . . 32 
4.2 .1 Quasiparticle Traps . . . . . . . . . . . . . . . . . . . 32

4.2 .2 Electrothermal Feedback . . . . . . . . . . . . . . . . . . 35

4.3 Charge Channels . . . . . . . . . . . . . . . . . . . . . . 37

Works Cited . . . . . . . . . . . . . . . . . . . . . . . . . . . . 39

\begin{tabular}{|lll}
5 & Development of CDMS II Detectors & 41
\end{tabular}

5.1 Detector Uniformity . . . . . . . . . . . . . . . . . . . . . 41

5.2 Normal and Parasitic Resistance. . . . . . . . . . . . . . . . . 43

5.3 Resistance vs. Temperature . . . . . . . . . . . . . . . . . . 43

$5.4 \quad$ Critical Current . . . . . . . . . . . . . . . . . . . . . . . . 47

5.5 IbIs - Sensor Current vs. Bias Current . . . . . . . . . . . . 47

Works Cited . . . . . . . . . . . . . . . . . . . . . . 53

6 Background Discrimination $\quad 55$

6.1 Event Reconstruction . . . . . . . . . . . . . . . . . . . 56

6.2 Search Thresholds . . . . . . . . . . . . . . . . . . . . . . . . . 59

6.3 Fiducial Volume Cut . . . . . . . . . . . . . . . . . . . . . . . . 60

6.4 Gamma Calibration . . . . . . . . . . . . . . . . . . . . . . . 64

6.5 Neutron Calibration $\ldots \ldots \ldots \ldots \ldots$

6.6 Population of the Bands in the Calibration Data . . . . . . . . . 66

Works Cited . . . . . . . . . . . . . . . . . . . . . . . . . . 71

\begin{tabular}{lll}
\hline & Analysis of Beta Contamination & 73
\end{tabular}

7.1 Selecting a Sample Population of Betas . . . . . . . . . . . . 74

7.2 Risetime as an Indicator of Depth . . . . . . . . . . . . . . . 76

7.3 Maximum Likelihood Fit for the Betas . . . . . . . . . . . . 78

7.4 Elimination of Betas in the NND Neutrons . . . . . . . . . . . . . 84

7.5 Elimination of Betas in the Single-Scatters . . . . . . . . . . . . 98

Works Cited . . . . . . . . . . . . . . . . . . . . . . . . . 103

8 Results and Conclusions $\quad 105$

8.1 Data Cuts and Their Efficiencies . . . . . . . . . . . . . 105

$8.2 \quad$ WIMP Candidates . . . . . . . . . . . . . . . . . . . . . . . . . . 109

$8.3 \quad$ Measuring the Neutron Background $\ldots \ldots \ldots \ldots \ldots$

8.4 Calculation of Cross-section/Mass Upper Limit . . . . . . . . . . . 114

8.5 Comparison with Other WIMP Searches . . . . . . . . . . . . 117

8.6 Outlook $\ldots \ldots \ldots \ldots \ldots \ldots \ldots$

Works Cited . . . . . . . . . . . . . . . . . . . . . . . . . 123 


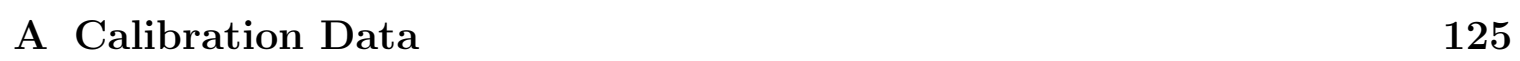

A.1 Search Thresholds. . . . . . . . . . . . . . . . . . . . . . . . . . . 125

A.2 Fiducial Volume Cut . . . . . . . . . . . . . . . . . . . . . 131

A.3 Gamma Calibration . . . . . . . . . . . . . . . . . . . . . 134

A.4 Neutron Calibration . . . . . . . . . . . . . . . . . . . . . . 141

๑. . . . . . . . . . . . . . . . . . . . . . . . . . . 149 


\section{List of Tables}

5.1 Detector names for CDMS Tower 1. . . . . . . . . . . . . . . . . . . . 42

$5.2 \quad$ Basic Phonon Sensor Properties for Detector Z5 (G9). . . . . . . . . . 44

6.1 Phonon and Ionization Search Thresholds . . . . . . . . . . . . . . . 60

$7.1 \quad$ Data fraction of neutrons and betas surviving a risetime cut . . . . . 78

7.2 The estimated data fraction surviving combinations of a position cut and a risetime cut after a Nuclear Recoil Band Cut. . . . . . . . . . . 88

7.3 The Number of Low-Yield, Nearest-Neighbor Double-Scatters in the Low-Background Data. . . . . . . . . . . . . . . . 95

7.4 Cut levels and Approximate Survival Percentages for Nearest-Neighbor Double-Scatter Neutrons. . . . . . . . . . . . . . . . . . . . 98

7.5 Estimated Neutron Survival Percentage in the Single-Scatter Events . 101

A.1 Estimated number of noise events misidentified as a signal given various search threshold levels (3V). . . . . . . . . . . . . . . 125

A.2 Estimated number of noise events misidentified as a signal given various search threshold levels $(6 \mathrm{~V}) . \ldots \ldots \ldots 130$ 


\section{List of Figures}

$2.1 \quad$ Evolution of comoving number density with respect to temperature . $\quad 9$

$3.1 \quad$ A Schematic of the Experimental Setup . . . . . . . . . . . . . . . . . 21

3.2 A Mechanical Drawing of the Dilution Refrigerator and the Icebox. . 25

3.3 A Mechanical Drawing of the Tower . . . . . . . . . . . . 26

$4.1 \quad$ A Photograph of a ZIP detector in its Housing. . . . . . . . . . . . . 30

$4.2 \quad$ A Sketch of the ZIP Detector Phonon Sensor . . . . . . . . . . . . . . 33

4.3 Superconducting Gap Structure of the Quasiparticle Traps . . . . . . 34

4.4 Typical resistance-vs.-temperature curve of a Phonon Sensor . . . . . 36

5.1 Simplified Circuit Diagram for the Phonon Sensor. . . . . . . . . . . . 45

5.2 Geometrical Sensitivity to $T_{c}$ of Resistance-vs.-Temperature and Critical Current Measurements. . . . . . . . . . . . . . . . . 46

5.3 Critical Current Curves for Detector Z5 (G9) . . . . . . . . . . . . . . 48

5.4 IbIs Curves for Detector Z5 (G9), Channel A (post-implant). . . . . . 50

5.5 Simulated $T_{c}$ Distribution for Z5 (G9) and Planned Implantation Scheme. 51

6.1 Sample Pulses for the Charge and Phonon Sensors for all Six Detectors. 57

6.2 Side View of the Fiducial Volume Cut . . . . . . . . . . . . . . . 61

6.3 A Sample Fit to the Fiducial Volume Cut . . . . . . . . . . . . . . . 62

6.4 Comparison of Over-corrected Crosstalk with Position . . . . . . . . . 63

6.5 Efficiency of the Fiducial Volume Cut versus Energy . . . . . . . . . . 63

6.6 A Sample ${ }^{60}$ Co Spectrum . . . . . . . . . . . . . . . . . 65

6.7 Uncertainties in the Band Widths. . . . . . . . . . . . . . . . 67

6.8 Electron Recoil and Nuclear Recoil Bands in the 3V Calibration Data 68

6.9 Electron Recoil and Nuclear Recoil Bands in the 6V Calibration Data 69

6.10 2-D Histograms of Yield vs. Energy in the Calibration Data . . . . . 70

$7.1 \quad$ Nearest-Neighbor Double-Scatter Betas at Low-Yield . . . . . . . . . 75

7.2 A Sample Phonon Pulse . . . . . . . . . . . . . . . . . . 76

7.3 Risetime-vs.-yield plot for calibration data . . . . . . . . . . . . . . . 77 
7.4 Data fraction of neutrons and betas surviving a risetime cut . . . . . 79

7.5 Energy Band Regions for the Beta Fits in Detector Z5 (Ge) . . . . . 82

7.6 A Sample Likelihood Fit for Betas in the Risetime-Yield Plane for Detector Z5 (Ge), from 40-60 keV] . . . . . . . . . . . . . . . . 83

7.7 Energy Band Regions for Detector Z5 (Ge) . . . . . . . . . . . . . . . 85

7.8 A Sample Likelihood Fit for Neutrons in the Risetime-Yield Plane for Detector Z5 (Ge), from 40-60 keV] . . . . . . . . . . . . 86

$7.9 \quad$ Distribution of the Discrimination Parameter $\mathcal{R}$ Integrated Over Rise-

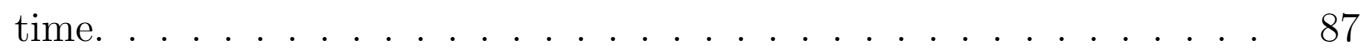

7.10 Distribution of the Discrimination Parameter $\mathcal{R}$ at High and Low Risetimes. . . . . . . . . . . . . . . . . . . . 88

7.11 Angular Separation of NND Betas and Neutrons . . . . . . . . . . . . 89

7.12 Parameter Space for Constructing a Conservative Beta Model . . . . 92

7.13 Risetime-vs.-Yield Distribution of the Conservative Beta Model . . . 93

7.14 A Model of Distribution of Angular Separations . . . . . . . . . . . . 94

7.15 Modeled Distribution of the Likelihood Function Ration, $\mathcal{R}$. . . . . . 96

7.16 Percentage of Events Passing a Cut in $\mathcal{R} \ldots \ldots$. . . . . . . . . . 97

7.17 Comparison of the Distribution of $\mathcal{R}$ Between Models and Data . . . 99

8.1 The distribution of nearest veto hits relative to random triggers. . . . 107

8.2 Combined Efficiency for Cuts in the Low-Background Germanium SingleScatters ....................... . . 110

8.3 Comparison of Efficiencies Between New (LFR) and Old (Flat) Cuts. 111

8.4 Distribution of Veto-Anticoincident, Low-Background, Single-Scatter Events in Z2, Z3, and Z5 in the Yield-vs.-Energy Plane . . . . . . . . 112

8.5 Comparison of WIMP Candidates using the Old and New Cuts . . . 113

8.6 A Yield-vs.-Yield Plot for Nearest-Neighbor Double Scatters in the Low-Background Data . . . . . . . . . . . . . . . . . . 115

8.7 Energy Spectrum of Single- and Multiple-Scatters in the Low-Background

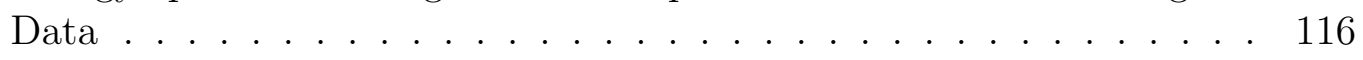

8.8 Spin-Independent WIMP-Nucleon Cross-section vs. WIMP Mass 90\% Confidence Level Upper Limits ․ . . . . . . . . . . . . . . . . . . . 118

8.9 Spin-Independent WIMP-Nucleon Cross-section vs. WIMP Mass 90\% Confidence Level Upper Limits . . . . . . . . . . . . . . . . . . . . . 119

A.1 Gaussian Fit of Phonon Noise Levels Based on Randomly-Triggered Data (3V). . . . . . . . . . . . . . . . 126

A.2 Gaussian Fit of Phonon Noise Levels Based on Randomly-Triggered Data (6V). . . . . . . . . . . . . . . . 127 
A.3 Gaussian Fit of Charge Noise Levels Based on Randomly-Triggered Data (3V). . . . . . . . . . . . . . . . . 128

A.4 Gaussian Fit of Charge Noise Levels Based on Randomly-Triggered Data (3V). . . . . . . . . . . . . . . . . 129

A.5 Definition of the $6 \sigma$ q-inner noise threshold and the $2 \sigma$ q-outer noise

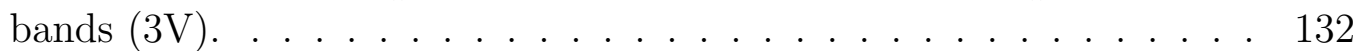

A.6 Definition of the $6 \sigma$ q-inner noise threshold and the $2 \sigma$ q-outer noise bands $(3 \mathrm{~V}) . \ldots \ldots \ldots \ldots \ldots \ldots \ldots$

A.7 Histograms of Yield in Slices of Energy in the Gamma Calibration Data, Detector Z1. . . . . . . . . . . . . . . . . . 135

A.8 Histograms of Yield in Slices of Energy in the Gamma Calibration Data, Detector Z2. . . . . . . . . . . . . 136

A.9 Histograms of Yield in Slices of Energy in the Gamma Calibration Data, Detector Z3. . . . . . . . . . . . . . . 137

A.10 Histograms of Yield in Slices of Energy in the Gamma Calibration Data, Detector Z4. . . . . . . . . . . . . . . . . 138

A.11 Histograms of Yield in Slices of Energy in the Gamma Calibration Data, Detector Z5. . . . . . . . . . . . . . 139

A.12 Histograms of Yield in Slices of Energy in the Gamma Calibration Data, Detector Z6. . . . . . . . . . . . . . . . . 140

A.13 Histograms of Yield in Slices of Energy in the Neutron Calibration Data, Detector Z1. . . . . . . . . . . . . . . . . . . . 142

A.14 Histograms of Yield in Slices of Energy in the Neutron Calibration Data, Detector Z2. . . . . . . . . . . . . . . . . . . . . 143

A.15 Histograms of Yield in Slices of Energy in the Neutron Calibration Data, Detector Z3. . . . . . . . . . . . . . . . . 144

A.16 Histograms of Yield in Slices of Energy in the Neutron Calibration Data, Detector Z4. . . . . . . . . . . . . . . . . . 145

A.17 Histograms of Yield in Slices of Energy in the Neutron Calibration Data, Detector Z5. . . . . . . . . . . . . . . . . 146

A.18 Histograms of Yield in Slices of Energy in the Neutron Calibration Data, Detector Z6. . . . . . . . . . . . . . . . 147 


\section{Acknowledgements}

First off, let me make the standard apology for anyone that I am forgetting. Everything in my life has led up to this moment, and everyone I've ever met had some part in getting me here. Sorry if I missed you...

First and foremost are, of course, my parents. Only now that I have children of my own do I understand some part of what my sister and I put them through. What can I say but, "Thank you." Thanks for letting me find my own way but kicking my butt when I needed it. Thanks for feeding me and clothing me (except for the sailor suits and corduroy pants) and making sure my shirt was tucked in and coaching my soccer team and dragging my bike everywhere and driving me across two states and typing in countless POKE statements from COMPUTE! magazine and making me tea and all of the other thousand things along the years. Any faults I have are my own, despite your better efforts.

Fast forward 24 years and here I am at Case. How I ended up sitting in the office of Dan Akerib talking about dark matter, I'll never know. Diana's Masters thesis advisor at Penn State knew someone at Case who was starting up a collider experiment and he suggested we check it out. I was doing computational relativity at the time, and had an interest in the theory program here. There wasn't room for 
me in theory and I wasn't too interested in solid-state physics, so Case was looking pretty low on my list until I went to pick up Diana at Dan's office and ended up talking with him about CDMS. What appealed to me then - and now! - was the scope of a particle-astrophysics experiment. How little did I know then how far from the computer terminal it would take me. Dan very consciously steered me away from programming tasks whenever he could, and so much of what I have learned as an experimentalist is thanks to his guidance. I've done plumbing, cryogenics, electronics, and even solid-state physics because he wouldn't let me turn into a terminal zombie. Much of my professional consciousness of the "human factor" is also due to his example. I hope all of his future students appreciate having him as an advisor. He let me get away with a lot, but not too much. I'm a far better all-around scientist because of him.

I remember very well entering our office in Room 101 for the first time and finding a cavernous space containing a single grad student sitting in front of a desk with a computer connected by a long, orange extension cord. Thushara and I became fast friends there, and I miss that time when he, Diana, and I would play Boggle on the computer over lunch. All too soon the office and the lab were transformed and online Boggle was lost to copyright law. We were soon joined by research associate Alex Bolozdynya, fellow grad students Gensheng Wang and Sharmila Kamat, and undergraduates Matt Truch and Moshe Katz-Hyman. What a diversity we had then! There's too much to say, but I can try to capture a snapshot each of our relationships in a short sentence: Alex - I always admired your work ethic - when you tightened a screw, it stayed put! Gensheng - thanks for introducing me to good tofu and mak- 
ing me think about more phonon physics than I probably would have on my own. Sharmila - thanks for putting up with Thushara and me, we just tried to treat you like one of the guys. Matt - thanks for introducing me to fantasy baseball (Diana doesn't thank you). Moshe - I really wanted to be the one installing Linux on a Mac $\mathrm{SE} / 30$ but I never had the time.

I've known Richard Schnee for the least amount of time, but in the last year our contact has been pretty intense. He has such an amazing grasp of so many different aspects of the experiment that I feel like an idiot every time I talk to him. As the "Analysis Guru," he is undoubtedly the person who has helped me the most in the creation of this document. More accurately, perhaps, would be to say that I've stuck to him like a leach and drained out of him as much I could. He has been a most gracious host, though, and he has always been patient and instructive with this stubborn and thick-headed student. If Dan has made me the scientist I am today, Richard is a reminder of how far I still have to go.

There are so many other people to thank. Thanks to Bruce Lambin, Rodney Choate, Bryan Johnson, Rich Schmitt, and all of the other folk who I bumped into in my time traveling to Soudan, MN. Special thanks go to Jim Beaty, who really takes care of all of us up in the Hinterland. Someday I hope he'll let me know which Lego character I was destined to be (I think!). Thanks to Aaron and Adam for taking on the thankless job of lab tech, thanks to Peter and Tim for helping out along the way, and thanks to Bryan and David and Brandon for taking the superuser password away from me (an knowing what to do with it!). Good luck to the newest additions to our team: Mike, Raul, and Cathy. I hope you learn as much from CDMS as much as I 
have.

I'd like to thank the CDMS collaboration as a whole for being an all-around great bunch of people. I continue to be amazed at the cohesiveness of a group that contains over 40 people at so many different institutions. It has been no small feat getting things as far along as they are, and I look forward - with some amount of jealousy - to seeing more of the great results which have already started coming out of the mine. A special note of thanks goes to Vuk Mandic for doing much of the initial development of the analysis method that eventually led to the results in this thesis.

Thanks to my thesis committee - Dan, Lawrence, John, and Heather - for making my defense a pleasant experience rather than the adversarial process that everyone fears. I always felt like you were trying to teach me rather than defeat me. I don't know how many other people can say that they actually enjoyed their defense. Thanks for putting up with my unorthodox schedule and many delays. It has been a long process for me and I appreciate your understanding.

Finally, let me thank my family for putting up with all of the grief I have put them through over the last two years or so. Thanks for my parents putting up with so many lost weekends and for Buni and Tata for giving me a basement hideaway and being with Diana and the kids when I couldn't. Diana, Astra, and now Marius have suffered much because of me. How can I pay you back for all of the travel and late nights, all of the missed holidays and birthday parties? Could I have done a better job balancing things? Could I have stayed up later? Could I have made better use of my time? The answer to all of these questions is almost certainly yes, but I hope you will forgive me for being the imperfect person that I am. Things will be better, 
I hope. At the same time, I'll never be able to pick up a copy of my thesis without thinking of The Forsyte Saga or of Uno and Sorry and The Magic School Bus or of watching Marius turn from a baby into a boy. Whatever hardships we suffered, there have been good times mixed in. To Diana, I can only say that I am sorry that you had the burden of carrying all of us across the finish line. I couldn't have done it without you, Love. 
Development and Performance of Detectors for the Cryogenic Dark Matter Search Experiment with an Increased Sensitivity Based on a Maximum Likelihood Analysis of Beta Contamination

\section{Abstract}

by

\section{Donald D. Driscoll, Jr.}

The Cryogenic Dark Matter Search (CDMS) uses cryogenically-cooled detectors made of germanium and silicon in an attempt to detect dark matter in the form of WeaklyInteracting Massive Particles (WIMPs). The expected interaction rate of these particles is on the order of $1 / \mathrm{kg} /$ day, far below the $200 / \mathrm{kg} /$ day expected rate of background interactions after passive shielding and an active cosmic ray muon veto. Our detectors are instrumented to make a simultaneous measurement of both the ionization energy and thermal energy deposited by the interaction of a particle with the crystal substrate. A comparison of these two quantities allows for the rejection of a background of electromagnetically-interacting particles at a level of better than $99.9 \%$. The dominant remaining background at a depth of $\sim 11 \mathrm{~m}$ below the surface comes from fast neutrons produced by cosmic ray muons interacting in the rock surrounding the experiment.

Contamination of our detectors by a beta emitter can add an unknown source of unrejected background. In the energy range of interest for a WIMP study, electrons will have a short penetration depth and preferentially interact near the surface. Some of the ionization signal can be lost to the charge contacts there and a decreased 
ionization signal relative to the thermal signal will cause a background event which interacts at the surface to be misidentified as a signal event. We can use information about the shape of the thermal signal pulse to discriminate against these surface events. Using a subset of our calibration set which contains a large fraction of electron events, we can characterize the expected behavior of surface events and construct a cut to remove them from our candidate signal events.

This thesis describes the development of the 6 detectors $(4 \times 250 \mathrm{~g}$ Ge and $2 \times 100 \mathrm{~g} \mathrm{Si}$ ) used in the 2001-2002 CDMS data run at the Stanford Underground Facility with a total of 119 livedays of data. The preliminary results presented are based on the first use of a beta-eliminating cut based on a maximum-likelihood characterization described above. 


\section{Chapter 1}

\section{Introduction}

This thesis reports on the culmination of my work as a graduate student with the Cryogenic Dark Matter Search (CDMS) from August 1997-December 2003. Much of what I have learned in that time is outside the storyline of this formal report, but is very important to my development as an experimental physicist and is reported here.

When I came to Case in August of 1997, the office space that CDMS now occupies contained one desk and one graduate student and our intended lab space was the former departmental "dumping ground," which had much earlier housed a Van der Graff accelerator. It was over a year of heavy lifting and hard work plus one trip to the emergency room before we had a habitable lab space and a working cryogenic system. A ${ }^{3} \mathrm{He} /{ }^{4} \mathrm{He}$ refrigerator sets its own timetable, though, and it was almost another year before we started producing useful data for the collaboration.

That early work consisted of measuring the transition temperatures of "witness samples," thin silicon substrates (1 mm vs. our $1 \mathrm{~cm}$ detectors, see Chapter 4) which are processed at the same time as our phonon sensors, in order to verify the $T_{c}$ gradient that we had suspected was present in our detectors. I would dice up these 3 -inch wafers into $3 \times 5 \mathrm{~mm}$ samples such that we could measure localized resistancevs.-temperature curves, thus mapping the distribution of $T_{c}$ across the surface. In addition to learning a lot about cryogenics in this time, I learned a lot about cryogenic wiring and low-temperature measurement. I developed a system for heatsinking and bringing down the wiring for these measurements from room temperature to base temperature $(\sim 15 \mathrm{mK})$. An important development that I devised was structuring this scheme such that four-wire resistance measurements could be made with the samples connected in series, allowing for 20 samples to be measured with a single DB25 cable. This allowed us the freedom to make a higher-resolution map of a wafer than had been previously feasible in other CDMS test facilities.

These measurements were made automatically via a GPIB-controlled resistance bridge using a LabView control program which I wrote from scratch. That program 
controlled the heating power provided to the fridge, measured the temperature of the fridge, and cycled through a resistance measurement of the samples. This same system also allowed us to calibrate thermometers which are now installed as monitoring in our fridge as well as in the refrigerator at our deep site in Soudan. Throughout our time making both of these types of measurements was an on-going battle with debugging the noise sources inherent in making low-temperature electrical measurements. More an art than a science, I have plugged and unplugged every single connector in our lab more times than I can count and have even resorted to wrapping things in aluminum foil!

Over the next year-and-a-half, the CWRU test facility slowly transitioned from making test sample measurements to testing CDMS physics detectors. After installing and debugging the CDMS electronics chain, we tested our first science-bound CDMS detector in January of 2001 (G6, which became referred to as Z1 when it was installed for detector running in the data run described in this thesis). This began a period of intense operation, with five of the six "Tower 1" detectors passing through the testing process described in Chapter 5 here at Case. A total of 21 detectors were tested in the last two years (2001-2002) that I was actively involved with the day-to-day operations of the test facility. During this time, the CDMS test facility also became involved with a joint U.S.-Russian project to develop CDMS-style cryogenic detectors in order to make a measurement of the anomalous magnetic moment of the neutrino suggested by the solar neutrino deficit (see http://nmm.case.edu/ for more details about this project).

Also during this time, the CDMS collaboration began to transition from the detector development and shallow-site running at Stanford University (see Chapter 3) to commissioning the deep site in Soudan, MN. In January 2002, I became one of the first physicists to take regular shift work at Soudan. The cryogenic systems had experienced some setbacks during installation, and as one of the collaboration's "cryoexperts," I became a part of the effort to debug and repair the ${ }^{3} \mathrm{He} /{ }^{4} \mathrm{He}$ refrigerator there. It took the better part of 2002 before we achieved nominal base temperature, and the struggles with the cryogenics systems continue to this day, although significant periods of stability allowed data-taking to begin in earnest.

Early in 2003, I stopped traveling to Soudan and I stopped being a regular part of the Test Facility team here at Case and began full-time analysis of the data described in this thesis. My original plan had been to wait for the first batch of Soudan data, but the continued cryogenic delays made that impractical. The final physics run at the shallow site in Stanford had been completed and the first half of that data had been analyzed as the Ph.D. thesis of Tarek Saab [1]. Half of that data remained unanalyzed, and while I had not taken part in the actual collection of this data, my part in the development of the detectors as well as analysis work that I began as early as mid-2001 gave me a claim to the data. 
After Tarek's graduation, I became the person primarily responsible for the analysis of this data, under the guidance of "Analysis Guru" Richard Schnee. I performed the detector characterization described in Chapter 6 as well as making incremental improvements in the method used to make that analysis. One of the important discoveries I made then was the presence of locations in our detector which were incorrectly connected (see Section 6.3), which we were then able to correct for subsequent detectors. The better part of the last year was comprised of the full development of the method used for eliminating a beta background described in Chapter 7, which was initiated earlier by Bernard Sadoulet and Vuk Mandic. This technique had not been performed before and is critical in improving the measurement of our neutron background in our shallow-site data. It will be even more important to our deep-site data when beta contamination will be our limiting background. 


\section{Works Cited}

[1] T. Saab. Search for Weakly Interacting Massive Particles with the Cryogenic Dark Matter Search Experiment. PhD thesis, Stanford University, August 2002. 


\section{Chapter 2}

\section{WIMPs}

\subsection{Dark Matter and Cosmology}

The dark matter problem has received much attention since it was posed 80 years ago when Fritz Zwicky observed the radial velocities of galaxies in the Coma Cluster [1. His measurements indicated that there was significantly more mass present than could be accounted for by the visible matter. Despite the fact Zwicky's calculations were based on an historically large Hubble constant [2], the discovery of a significant non-visible component of the universe has dramatically changed our understanding of cosmology.

As late as the 1970s, scientists were still struggling to reconcile the conflict between astronomical observations and Newtonian gravity. Rubin and Ford [3] made one of the most convincing observations in 1970 when their measurements of the nearby galaxy M31 showed that the mass inferred from the motions of ionized gas in the disk was about 10 times the mass inferred by the galaxy's luminosity. Since M31 is in the Local Group of galaxies and is less than $1 \mathrm{Mpc}$ away from the Earth [4], these measurements were not as dependent on cosmological parameters as measurements of more distant galaxies. This finding made it apparent that either our understanding of galactic physics was flawed, or there was some missing mass, or dark matter, present.

Today, most scientists are not as concerned with proving of the existence of dark matter, but rather understanding its nature. We are currently entering a golden age of astrophysics in which a wealth of observational data promises to answer a number of long-standing cosmological problems, not the least of which is the nature of dark matter. Precision measurements of anisotropies in the Cosmic Microwave Background (BOOMERANG [5], DASI [6], MAXIMA [7]) indicate that the energy density of the universe is close to the critical value for flatness $\left(\Omega_{t o t} \sim 1\right)$. Both big-bang nucleosynthesis (BBN) and observational measurements tell us that only about $5 \%$ of 
this critical value can be composed of baryons $\left(\Omega_{b} \sim 0.05 \Omega_{t o t}\right)$. When combined with information about the large-scale structure of the universe, such as the measurement of high-z supernovae [8], we see that the the remainder of the universe is composed of a $20 \%$ dark matter component and a $75 \%$ dark energy component. When combined with the recent results of the WMAP satellite [9], these observations overwhelmingly confirm that the universe is flat, and can be described by the $\Lambda \mathrm{CDM}$ (Cold Dark Matter with a Cosmological Constant, $\Lambda$ ) model of the universe. While the dark energy problem is even more puzzling, we are now convinced that the dark matter is there. There is even a hope that we can determine what it is.

Over the years, there have been many candidates for what this non-luminous matter could be. Early hypotheses included large quantities of hot gas or dust in the interstellar medium. One promising candidate was the MACHO, or MAssive Compact Halo Object [10]. These objects are roughly solar-mass objects which populate the halo of the galaxy but are not self-luminous, such as brown dwarfs ("stars" which are not massive enough to start fusion), burnt-out white dwarfs, and black holes. While all of these objects are non-luminous and probably exist in some amount, none of them have provided more than a few percent of the apparent deficit. Moreover, all of these objects were created from baryons, so we must look to particle physics to provide us with a non-baryonic dark matter candidate.

\subsection{Particle Dark Matter}

It is useful to understand some of the basic properties of particle dark matter in general before discussing a direct detection experiment. One of the first papers to suggest that a massive dark matter particle could have a significant relic density was published by Lee and Weinberg in 1977 [11]. Prior to this paper, the upper limit for a the mass of a "stable neutral heavy lepton" was considered to be

$$
m_{L}<\frac{\rho}{n}=\frac{2 \times 10^{-29} \mathrm{~g} / \mathrm{cm}^{3}}{300 / \mathrm{cm}^{3}} \approx 40 \mathrm{eV}
$$

where $\rho$ is the upper limit for the mass density of the universe and $n$ is the average number density at the present time [12]. This estimate was based on a density of

particles which was about half that of the cosmic microwave background photons, as would be expected for a neutrino with a small mass.

Any type of particle can maintain chemical equilibrium in the early universe since there is enough energy on average to pair-produce particles at the same rate at which they are annihilated. They also maintain thermal equilibrium through collisions with the rest of the plasma. Collisions between particles become rare due to the expansion of the universe and different types of particles "freeze out" at a number density for 


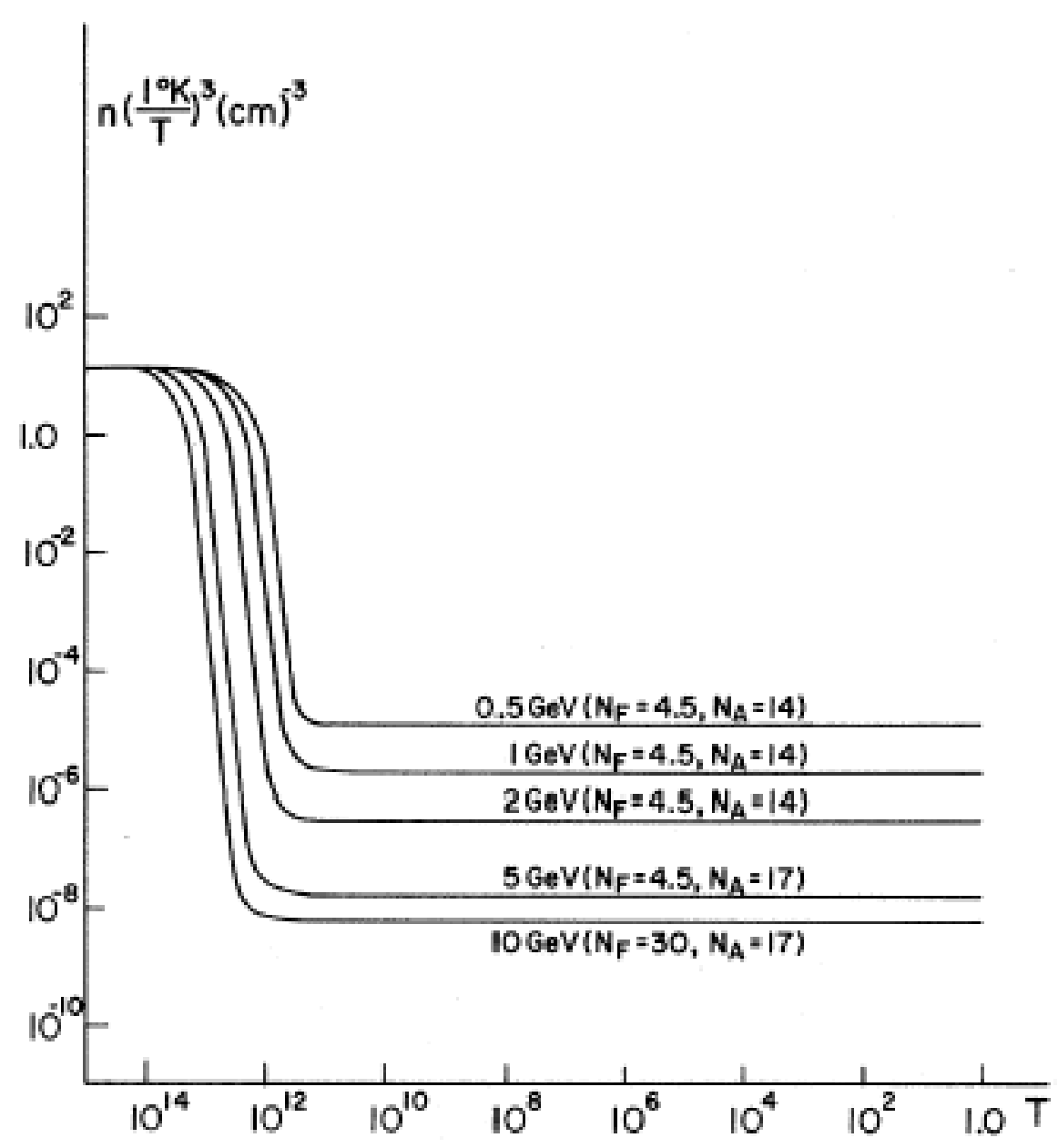

Figure 2.1: Evolution of comoving number density with respect to temperature. Taken from Lee \& Weinberg (1977) [11].

which their annihilation cross-section yields a vanishing interaction rate.

Lee and Weinberg showed that particles whose mass is greater than about $1 \mathrm{MeV}$ drop out of chemical equilibrium before freeze-out. As the universe cools, the average energy drops below the $2 M$ needed for pair-production, but the particles continue to self-annihilate until freeze-out. The present number density can then be significantly smaller than the CMB photons, allowing for a more massive dark matter particle in Equation 2.1. A graphical representation of this process is given in Figure 2.1.

Thus, the number density of particles which survive is governed by two quantities. First, the time at which the particle drops out of chemical equilibrium depends of the mass of the particle. The temperature of freeze-out depends on the annihilation cross-section of the particle since it defines the rate of annihilation given a 
particular number density. Given a particle mass and cross-section, it is possible to compute a relic density of that particle. As an example, we follow Lee and Weinberg's formulation for computing the relic density (Equation 2.2) of a heavy neutrino:

$$
\rho_{L}=\left(4.2 \times 10^{-28} \mathrm{~g} / \mathrm{cm}^{3}\right)\left(\frac{m_{L}}{G e V}\right)^{-1.85}\left(\frac{N_{A}}{\sqrt{N_{F}}}\right)^{-0.95} \text { [11] }
$$

where $m_{L}$ is the particle's mass, $N_{A}$ is a number which depends on the annihilation channels available, and $N_{F}$ is the number of degrees of freedom, with $\frac{N_{A}}{\sqrt{N_{F}}} \sim 7$. The dependence on cross-section is included in the prefactor, assuming a charged-current Fermi interaction. An interesting computation is to use the WMAP value for the density of dark matter, $\rho_{L} \sim 0.22 \Omega \sim 4.4 \times 10^{-30} \mathrm{~g} / \mathrm{cm}^{3}$, which yields $m_{L} \sim 5 \mathrm{GeV}$.

\subsection{WIMP Dark Matter}

The paper by Lee and Weinberg specifically discussed a heavy neutrino as their candidate particle, but a number of particles fall into a general class of particles with similar properties, called "WIMPs," or Weakly Interacting Massive Particles. These particles are weakly-interacting in that their cross-sections are very small, not that they are mediated by the weak force. As we see below, $\sigma \ll 10^{-40} \mathrm{~cm}^{2}$ for most masses (10-1000 GeV). The Standard Model of particle physics predicts no massive particle with such properties, but many well-motivated extensions to the Standard Model do.

In general, Supersymmetric (SUSY) extensions to the Standard Model are an attempt to reconcile the fact that the masses of particles are not stable to radiative corrections. Processes which emit and reabsorb a virtual particle, for example, add terms which contribute to the particle's mass. Infinite combinations of these processes lead to particles with infinite mass. These terms can be cancelled by giving the particle an infinite bare mass, but this mass must cancel the radiative corrections at the level of 32 significant figures [13]. A more natural solution to this problem is to propose "symmetric" particles such that for every boson in the Standard Model there would exist a "partner" fermion and for every fermion there would exist a "partner" boson. These partners would have similar masses and couplings as their Standard Model counterparts (prior to spontaneous symmetry breaking), but would differ by a spin of $1 / 2$. This introduces a relative negative sign in the radiative correction terms such that the "partner" terms cancel out the "standard" terms at the electroweak scale according to the similarity of their masses and couplings, thereby allowing for the finite masses that we observe.

The Minimal Supersymmetric Standard Model (MSSM) attempts to include these particles by adding as little as possible to the Standard Model. Even in this simplest 
model, there are allowed interactions which could violate both baryon number $(B)$ and lepton number $(L)$. This would lead to (currently) unobserved physics such as proton decay. A proposed parity of this system, called the R-parity

$$
R=(-1)^{(3 B+L+2 s)}
$$

could prevent such interactions. This quantity was constructed such that $R=1$

for all standard model particles ${ }^{1}$, while the superpartners would have the same $B$ and $L$ but would differ by $\Delta s=\frac{1}{2}$ such that $R^{\prime}=-R=-1$. If this parity is conserved, the the lightest supersymmetric partner (LSP) would be stable much the way that conservation of baryon number implies a stable proton. A stable, massive LSP provides us with an ideal dark matter candidate.

\subsection{Direct Detection of WIMPs}

A seminal paper on direct detection was written by Goodman and Witten [14] and considered the detection of "three classes of dark-matter candidates: particles with coherent weak couplings; particles with spin-dependent couplings of roughly weak strength; and particles with strong interactions." The first type, particles with coherent weak couplings, is particularly relevant to a WIMP search. It exploits the fact that when a WIMP scatters off of a nucleus, the scattering process takes place via a WIMP-quark interaction. An interaction between a WIMP and a nucleus with radius $r_{n}$ can scatter coherently if the wavelength corresponding to the momentum transfer is much less than the nuclear radius $\left(q \ll \frac{h}{r_{n}}\right)$ [12. The resulting WIMP-nucleus crosssection is then found from the coherent sum of the scattering amplitudes of all the quarks in the nucleus, which produces an enhanced cross-section. Since we compare experiments using different target nuclei, the summation over quarks in a nucleon (proton or neutron) is done implicitly, while the summation over nucleons in a nucleus is left in terms of atomic weight, $A$. For coherent scattering, the WIMP-nucleus cross-section is enhanced by a factor $A^{2}$ relative to the WIMP-nucleon cross-section [12] since the number of scatterers (quarks) is proportional to A. This enhancement is only applicable to spin-independent interactions since terms from oppositely-directed spins cancel out in a spin-dependent interaction.

Goodman and Witten derived a formula for the WIMP-nucleon cross-section on the order of $\sigma_{n} \sim 10^{-35} \mathrm{~cm}^{2}$ for WIMPs of mass $m_{W}<10^{6} \mathrm{GeV}$. This suggested that interaction rates for coherent weak couplings greater than a detection per day

\footnotetext{
${ }^{1} B=1, L=0, s=\frac{1}{2} \Rightarrow R=+1$ for a proton; $B=0, L=0, s=1 \Rightarrow R=+1$ for a photon; $B=0, L=-1, s=\frac{1}{2} \Rightarrow R=+1$ for an electron, etc.
} 
could be possible using a kilogram of detector mass. We can make a similar estimate of the detectability of WIMPs based an assumed set of astrophysical and interaction parameters following the formulation of Lewin and Smith [12]. These values are neither the current, most-accepted values for these parameters nor the only values consistent with observation, but rather they are chosen as "standards" because they are based on a simple model and allow for a uniform comparison between experiments.

First, we assume that the dark matter is distributed in an isothermal halo about the galaxy. As the sun revolves about the center of the galaxy and the earth revolves around the sun, we pass through a cloud of WIMPs which has a characteristic velocity of $v_{0} \approx 230 \mathrm{~km} / \mathrm{s}$ relative to an earthborn target and a local density of $\rho=0.3 \mathrm{GeV} \mathrm{cm}^{-3}$. These two quantities give us the flux of particles, for example:

$$
\Phi=\frac{\rho v_{0}}{m_{W}} \sim 7 \times 10^{5} s^{-1} \mathrm{~cm}^{-2}
$$

for a $100-G e V$-mass WIMP. We can then estimate an interaction rate per kilogram of detector of:

$$
R=\Phi N \sigma_{N}=\Phi \frac{N_{A}}{A} \sigma_{N}
$$

where $N$ is the number of target nuclei per unit mass and $\sigma_{N}$ is the cross-section for WIMP-nucleus interactions. The current upper limit for the WIMP-nucleon cross-section of a $100 \mathrm{GeV}$ WIMP test particle (see Figure 8.9) is of the order $\sigma_{n}=10^{-42} \mathrm{~cm}^{2}$. This WIMP-nucleon cross-section for a coherent, spin-independent interaction can be calculated from the WIMP-nucleus cross-section via [15]:

$$
\sigma_{N}=\frac{\mu_{N}^{2}}{\mu_{n}^{2}} A^{2} \sigma_{n}
$$

where $\mu_{N}$ and $\mu_{n}$ are the reduced masses of the WIMP-nucleus and WIMP-nucleon systems and $A$ is the atomic weight of the target nucleus.

Combining Equations 2.4, 2.5, and 2.6, an estimate of the interaction rate for such a particles is:

$$
R=\left(\frac{\rho v_{0}}{m_{W}}\right)\left(\frac{N_{A}}{A}\right)\left(\frac{\mu_{N}^{2}}{\mu_{n}^{2}}\right) A^{2} \sigma_{n}
$$

We can find the maximal event rate for a given target nucleus by choosing the case where the WIMP and the target nucleus have approximately the same mass, $\mu_{N} \sim$ $m_{W} / 2$ and $\mu_{n} \sim m_{N} / A \sim m_{W} / A$ :

$$
R_{\text {max }} \sim \rho v_{0} N_{A} A^{2} \sigma_{n} \sim 1 / k g \cdot d y
$$

for $m_{W} \sim 100 \mathrm{GeV}$, which gives us the desired rate of about one detection per day. 
There are a few points of interest in this last calculation, which is only valid at the maximally sensitive point $m_{N}=m_{W}$. First, it reproduces the general result that the interaction rate goes as $A^{2}$ for a coherent scatter, which strongly suggests using a high-A material as our detector. Looking more carefully at Equation 2.6, we see that:

$$
\frac{\mu_{N}^{2}}{\mu_{n}^{2}}=\left(\frac{m_{N} m_{W}}{m_{N}+m_{W}}\right)\left(\frac{m_{n}+m_{W}}{m_{n} m_{W}}\right)=\frac{A\left(m_{n}+m_{W}\right)}{\left(A m_{n}+m_{W}\right)}
$$

where we have substituted $m_{N}=A m_{n}$. For $m_{n} \ll m_{W} \ll m_{N}$, we have that

$R \propto \frac{A^{3}}{m_{N}}=\frac{A^{2}}{m_{n}}$ but for $m_{N} \ll m_{W}$, we have $R \propto \frac{A^{3}}{m_{W}}$, which is another reason to use a high-A material.

We would then expect that the interaction rate in an experiment, expressed in terms of the minimum detectable cross-section, would be flat for masses below $m_{N}$ and would decrease as $\sigma \propto m_{W}$ for higher masses. We have not yet discussed how much energy is deposited in the detector. We expect our WIMP spectrum to be an exponential with a cutoff energy [12, so the energy threshold of our experiment is critical in determining the detectable rate. For WIMP masses below $m_{N}$, the threshold is the limiting factor in the sensitivity. There is an additional loss of sensitivity for high WIMP masses due to the loss of coherence when the deBroglie wavelength of the WIMP becomes smaller than the size of the nucleus. These effects can be compactly expressed as:

$$
\left.\frac{d R}{d E}\right|_{\text {obs. }}=R_{0} S(E) F^{2}(E) I
$$

where $R_{0}$ is the theoretical limit given a perfect detector and a stationary Earth [12]. $S(E)$ takes into account the averaged motion of the Earth through the halo as well as any detector-dependent physics such as the detection efficiencies or energy thresholds. $F^{2}(E)$ is a form factor which depends on the target nucleus and the physics of the interaction as well as integrating the momentum transfer over all possible scattering angles.

\subsection{The CDMS Approach}

Any dark matter search must address several difficulties. First and foremost is the background rate. Any attempt to detect a signal on the order of one-per-day must have a clear understanding of any sources of background and remedies must be put in place to reduce or reject them. One of the obvious first steps is to locate the experiment underground since the rate of cosmic rays at the surface of the earth will easily overwhelm a WIMP signal. It was decided that the first phase of the CDMS experiment would be performed at a relatively shallow site since there are additional 
complications associated with building a complex experiment deep underground. This would allow us to more easily develop the technology for our detectors with a somewhat reduced background before moving to a deeper site. Even underground, there are many sources of background which remain, including natural radioactivity from the rock surrounding the experiment, cascading particles caused by residual muon collisions in the overburden, and contamination with radioactive isotopes via cosmogenic activation or during detector handling. Chapter 3 is primarily concerned with the construction of an apparatus designed to reduce these backgrounds. It includes an active muon veto, passive lead and polyethylene shields, and a careful choice of materials.

The measured background rate after this shielding is still two orders of magnitude above the target WIMP rate[16, but most of this background can be rejected since the physics of the energy deposition in the detector is different for particles which interact with the electron cloud than those which interact with the nuclei. Both types of events deposit energy by liberating electron-hole pairs (ionization) as well as increasing the temperature of the detector through crystal lattice vibrations in our semiconductor targets. Nuclear recoil events produce a larger fraction of thermal energy when compared with an electron recoil of the same energy. Electromagnetically-interacting particles such as electrons and photons are most likely to interact via an electron recoil, while WIMPs and neutrons are more likely to interact as nuclear recoils. The CDMS detectors, described in Chapter 4, are either germanium or silicon disks which are instrumented to make a simultaneous measurement of both the ionization energy and thermal energy of the collision. The ratio of these quantities, which we call the charge yield, allows us to reject over $99.9 \%$ of the photon background. Note that both neutrons and WIMPs cause nuclear recoils. We will see that neutrons - particularly those produced by cosmic ray muons - will be the limiting background at our shallow site.

The thermal energy of an interaction is measured by a superconducting thin film on one surface of our detectors. Fabrication of this phonon sensor is a very sensitive process, and we have had an average yield of less than $50 \%$ leading up to the detectors used in this data running[17]. Chapter 5 describes the testing program we have developed to assure that our detectors are suitable for a dark matter search. Once a detector is ready for use, it is subjected to a gamma calibration source $\left({ }^{60} \mathrm{Co}\right)$ and a neutron calibration source $\left({ }^{252} \mathrm{Cf}\right)$ to define the electron recoil and nuclear recoil bands in the yield-vs.-recoil energy plane. Chapter 6 discusses the separation of these two bands and the estimated rejection of our photon background.

We will see in Chapter 3 that one potentially troubling source of contamination is a beta emitter. Since electrons have a very short penetration depth, they preferentially interact at the surface of the detector. Surface events can lose some of their ionization energy to the charge contacts, leading to a decreased separation between electron 
recoils and nuclear recoils. Chapter 7 describes a method of eliminating surface events from our signal region by including information about the pulse shape of the phonon signal. Having eliminated most of the gamma and beta background events, we can use knowledge of our neutron background to make a statistical estimate of how many of the remaining candidate events could be WIMPs. In Chapter 8, we calculate an upper limit on the cross-section of a WIMP of a given mass based on these events. 


\section{Works Cited}

[1] F. Zwicky. Helv. Phys. Acta, 6:110, 1933.

[2] S. van den Bergh. The early history of dark matter. Publications of the Astronomical Society of the Pacific, June 1999. astro-ph/9904251.

[3] V.C. Rubin and W.K. Ford, Jr. Rotation of the andromeda nebula from a spectroscopic survey of emission regions. The Astrophysical Journal, 159:379403, February 1970.

[4] M.V. Zombeck. Handbook of Astronomy and Astrophysics, Second Edition. Cambridge University Press, 1990.

[5] C.B. Netterfield et al. A measurement by BOOMERANG of multiple peaks in the angular power spectrum of the cosmic microwave background. Feb 2002. astro-ph/0104460.

[6] N.W. Halverson et al. DASI first results: A measurement of the cosmic microwave background angular power spectrum. ASTROPHYSICAL JOURNAL, 568(38), May 2002. astro-ph/0104489.

[7] A.T. Lee et al. A high spatial resolution analysis of the MAXIMA-1 cosmic microwave background anisotropy data. April 2001. astro-ph/0104459.

[8] S. Perlmutter et al. Measurements of $\omega$ and $\lambda$ from 42 high-redshift supernovae. The Astrophysical Journal, 517:565-586, June 1999.

[9] C.L. Bennett et al. First year Wilkinson Microwave Anisotropy Probe (WMAP) observations: Preliminary maps and basic results. 2003.

[10] David Bennett. Magellanic cloud gravitational microlensing results: What do they mean? August 1998. astro-ph/9808121.

[11] B.W. Lee and S. Weinberg. Cosmological lower bound on heavy-neutrino masses. Physical Review Letters, 39(4):165-168, July 1977.

[12] J.D. Lewin and P.F. Smith. Review of mathematics, numerical factors, and corrections for dark matter experiments based on elastic nuclear recoil. Astroparticle Physics, 6:87-112, 1996.

[13] K.A. Olive. Introduction to supersymmetry: Astrophysical and phenomenological constraints. November 1999. hep-ph/9911307, based on lectures delivered at the Les Houches Summer School, July 1999. 
[14] M.W. Goodman and E. Witten. Detectability of certain dark-matter candidates. Physical Review D, 31(12):3059-3063, June 1985.

[15] Sunil Ramanlal Golwala. Exclusion Limits on the WIMP-Nucleon ElasticScattering Cross Section from the Cryogenic Dark Matter Search. PhD thesis, The University of California at Berkeley, Fall 2000.

[16] D. Abrams et al. Exclusion limits on the wimp-nucleon cross section from the cryogenic dark matter search. Physical Review D, 66, 2002.

[17] Paul Brink et al. Detector technical review. Internal note, August 2001. 


\section{Chapter 3}

\section{The CDMS Experiment}

As we saw in the previous chapter, we are trying to detect an interaction which occurs on the order of once per day per kilogram of detector mass. The main issue in designing a dark matter experiment is to reduce the rate of background events to the point that such an infrequent signal can be unambiguously detected. At the surface of the earth, cosmic rays provide a significant background of particles. Locating the experiment underground reduces this background via the shielding of the earth itself. The remaining backgrounds can be roughly categorized by particle type: gamma backgrounds, neutron backgrounds, and beta backgrounds. The gamma background can be rejected by our detectors at a level of better than $99.9 \%$, but we still must reduce it to the point that misidentification is negligible when compared to other backgrounds. The neutron background is of primary concern since it mimics a WIMP signal and we must take all possible steps to eliminate it. A beta background would not seem to be a problem since an electron interacts electromagnetically just as a photon does, but electrons with energies comparable to WIMP-induced recoils do not penetrate deeply into the detectors. In Chapter 4 , we see that these so-called surface events can be misidentified as a nuclear recoil since they will lose some of their ionization signal into the charge contacts. The analysis developed in later chapters is focused on identifying and rejecting betas based on their pulse shape. Understanding this background will be especially important in the future since it is believed that this background could be a limiting factor in data taken at our deep site.

\subsection{Cosmic Ray Backgrounds}

The first phase of the CDMS experiment was performed at the Stanford Underground Facility (SUF) located 11 meters below the surface of the Stanford University campus. This provides a shielding from cosmic rays equivalent to 16 meters of water, 
which is sufficient to stop the hadronic component of the cosmic rays and reduce the muon component by a factor of 5 [1]. The remaining flux of vertical muons at SUF $\left(\sim 29 \mathrm{~m}^{2} \mathrm{~s}^{-1} \mathrm{sr}^{-1}[1]\right)$ can be effectively rejected by constructing an active shield composed of plastic scintillator and photomultiplier tubes, shown schematically in Figure 3.1. We have seen that our veto shield has a time-averaged efficiency of $99.9 \%$, rejecting an event rate of approximately $6 \mathrm{kHz}$ [1]. The threshold for the veto is set such that minimum-ionizing muons $(\sim 8 \mathrm{MeV})$ are rejected, but the high rate of lower-energy gammas $(<3 \mathrm{MeV})$ does not dominate the trigger rate. The resulting deadtime is about $15 \%$.

In addition to the primary cosmic rays, collisions in the material both inside and outside of the experimental volume can create secondary showers of particles of all the types listed below. Muon-induced particles within the veto shield are rejected by coincidence with the muon's passage through the shield. Particles created outside the veto must be shielded according to the strategies listed below.

\subsection{Gamma Backgrounds}

The background of photons is dominated by natural radioactivity in the surrounding rock. The Earth's crust contains trace amounts of ${ }^{40} \mathrm{~K}$ as well as ${ }^{238} \mathrm{U},{ }^{232} \mathrm{Th}$, and their daughter isotopes. The ${ }^{40} \mathrm{~K}$ produces a $1.46 \mathrm{MeV}$ photon via electron capture, while the ${ }^{238} \mathrm{U}$ and ${ }^{232} \mathrm{Th}$ chains provide a number of different decay products whose abundance can theoretically be measured by identifying by their characteristic lines. We shield our detectors against a photon background by surrounding them with lead. Monte Carlo simulations of this shielding show that a $15 \mathrm{~cm}$ thickness of lead is the maximally useful amount since the background is then dominated by radioactivity from the ${ }^{210} \mathrm{~Pb}$ isotope present in modern lead[2].

Recently-mined lead contains a natural abundance of radioactive isotopes, most notably ${ }^{210} \mathrm{~Pb}$ which has a 22 -year half-life. ${ }^{210} \mathrm{~Pb}$ in the shield beta-decays to ${ }^{210} \mathrm{Bi}$ (and subsequently to ${ }^{210} \mathrm{Po}$ ) and the ejected electron produces a Bremsstrahlung spectrum with an endpoint of $1.16 \mathrm{MeV}$ [3]. In order to reduce this background, the inner $5 \mathrm{~cm}$ of the shield was composed of older lead ("Glover lead"), which has a significantly reduced amount of ${ }^{210} \mathrm{~Pb}$. Surrounding the detectors is a separate, 1-cm-thick inner shield made from of ancient lead ("Nantes lead") which has almost no ${ }^{210} \mathrm{~Pb}$ contamination.

With this shielding scheme in place, a previous analysis ("Run 19" - [1]) measured a background of photons of $60 \mathrm{keV}^{-1} \mathrm{~kg}^{-1} \mathrm{dy}^{-1}$ with an approximately exponential spectrum with a decay constant of the order $\tau \sim 70 \mathrm{keV}$ [4]. Of this background, about $2 \mathrm{keV}^{-1} \mathrm{~kg}^{-1} \mathrm{dy}^{-1}$ were anticoincident with a muon passing through the veto shield. This remaining event rate must be due to some amount of radioactive material close 


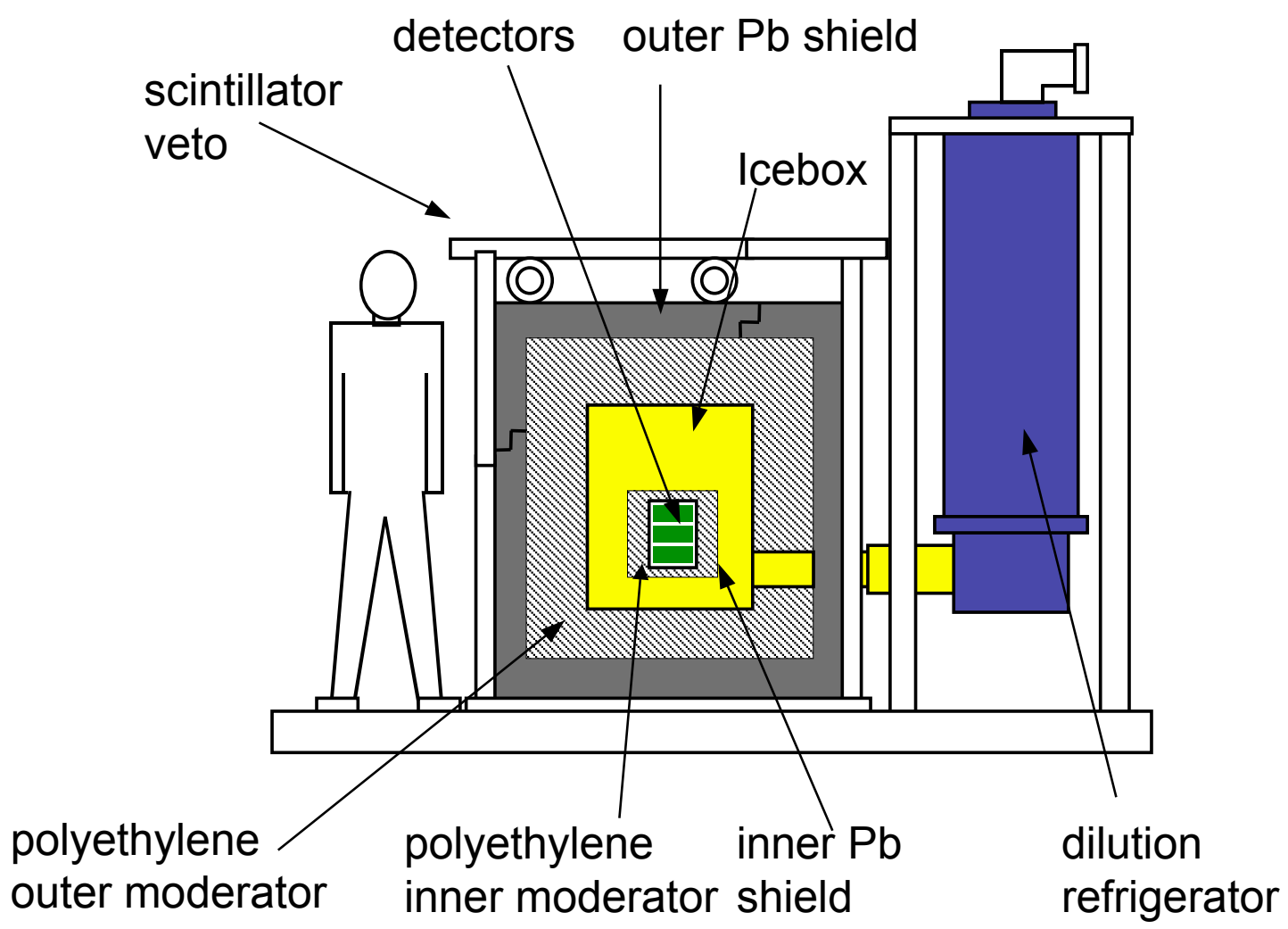

Figure 3.1: A Schematic of the Experimental Setup. On the right is the dilution refrigerator, a KelvinOx 400-S with a nominal cooling power of $400 \mu \mathrm{W}$ at $100 \mathrm{mK}$. This is connected to our experimental chamber ("The Icebox") via a copper "cold finger." The Icebox is made of high-purity copper and has thermally isolated vacuum spaces at each of the heatsinking layers of the refrigerator. The Icebox contains our detector package ("The Tower" - see Figure 3.3) and two layers of internal shielding. The outer shielding comprises a 2-meter cube and is surrounded by plastic scintillator which is instrumented as a muon veto shield. 
to the detectors, either in the inner (ancient) lead or the detector instrumentation itself.

\subsection{Neutron Backgrounds}

One source of our neutron background is natural radioactivity in the rock surrounding the experiment. Cosmic rays can also produce neutrons both directly via collisions as well as indirectly via the capture of negative muons on a nucleus through the reaction:

$$
\mu^{-}+\mathrm{p} \rightarrow \nu_{\mu}+\mathrm{n}
$$

Our lead shielding is not effective at stopping neutrons since the maximum momentum transfer for elastic scattering occurs when the two objects have the same mass. A lead atom is over 200 times as massive as a neutron, so after a scatter a neutron changes direction but does not lose energy. In order to be an effective moderator of neutrons, a material needs to have a high density of a low-Z material. Polyethylene is commonly used as a neutron moderator since it is composed of chains of ethylene $\left(\mathrm{C}_{2} \mathrm{H}_{4}\right)$ and contains many hydrogen atoms (with single-proton nuclei). Monte Carlo simulations indicate that a $25 \mathrm{~cm}$-thick layer of polyethylene placed between the inner and outer lead shields attenuates this neutron flux by a factor of almost 1000 [4].

The ambient neutron flux at SUF was measured with a $\mathrm{BF}_{3}$ detector to be $(8.1 \pm 0.6) \times 10^{5}$ neutrons $\mathrm{cm}^{-2} \mathrm{sec}^{-1}$ [4]. Conservatively assuming that all 23 events from the $15.8 \mathrm{~kg}$ dy of exposure reported in [1] were neutrons gives us a neutron background of less than $2 \mathrm{~kg}^{-1} \mathrm{dy}^{-1}$. Further Monte Carlo simulations showed that the addition of a layer of polyethylene shielding inside the inner lead shield could provide a reduction of this residual neutron flux by about a factor of 2 [4]. The previous analysis of the CDMS data taken at SUF (20 nuclear recoil events in $28.3 \mathrm{~kg}$ dy) is in good agreement with the simulations [5].

This residual neutron background is dominated by secondaries from high-energy

neutrons produced by muon interactions outside the veto 44. These neutrons are produced in evaporative processes, that is, from collisions which deposit energy directly into the nucleus. This can increase the intra-nuclear temperature to the point that a neutron is ejected. These "fast" neutrons have energies high enough to travel through the polyethylene shield, where they can then collide with a nucleus inside the shielding and eject a lower-energy neutron. It is estimated that about $40 \%$ of these neutrons are rejected by interactions of the fast neutrons in the muon veto [4], but some fraction of the unvetoed neutrons deposit energy in the detectors. This source of background will be greatly reduced when we start taking data deep underground, where the additional rock will attenuate the muon flux and fewer fast neutrons will 
be produced.

\subsection{Beta Backgrounds}

A subtle and hard-to-track form of radioactive contamination comes from the handling of the detectors. Three sources of contamination are particularly important, ${ }^{210} \mathrm{~Pb}$, ${ }^{14} \mathrm{C}$, and ${ }^{40} \mathrm{~K}$, since they are beta emitters. The latter two can be deposited by contact with human skin and other organic materials such as room dust. Care has been taken to limit contact with the detectors through protective clothing and strict handling protocols.

We do not expect lead atoms to migrate onto the detectors from the shield, but exposure to the atmosphere can cause radon to plate out onto the surface of our detectors. Radon is part of the uranium decay chain and can produce a multitude of long-lived radioactive daughters, including ${ }^{210} \mathrm{~Pb}$. We reduce presence of these contaminants by limiting the exposure of our detectors to the atmosphere. During experimental running, they are kept under vacuum. They are only exposed to the atmosphere for a short time while being removed or inserted from the experiment and are immediately returned to an over-pressured storage cabinet. This cabinet protects the detectors from dust, and boil-off from a liquid nitrogen dewar is flowed through the cabinet to provide a radon-free environment.

\subsection{Cosmogenic Activation}

One final source of contamination bears note. Our detector storage cabinet is kept underground in an adjacent tunnel at SUF to limit activation of materials from cosmic rays. Interactions of cosmic rays with ${ }^{73} \mathrm{Ge}$ atoms can produce ${ }^{68} \mathrm{Ge}$ and ${ }^{65} \mathrm{Zn}$, both of which emit low-energy x-rays $(\sim 10 \mathrm{keV})$. Neutrons from cosmic ray interactions as well as from our own neutron calibrations can activate ${ }^{71} \mathrm{Ge}$ and ${ }^{73 \mathrm{~m}} \mathrm{Ge}$ isotopes. ${ }^{71} \mathrm{Ge}$ decays to ${ }^{71} \mathrm{Ga}$, emitting a characteristic $10.4 \mathrm{keV}$ photon. Much of our detector packaging is constructed from high-purity copper (see below), which is also subject to cosmogenic activation. We have seen evidence of three $\sim 10 \mathrm{keV}$ x-rays caused by neutron and cosmogenic activation in our detectors following exposure to our neutron calibration source [6].

\subsection{Materials and Construction}

Throughout the construction of a low-background experiment, we must be extremely careful in our choice of materials. We have gone to such great lengths to reduce 
our backgrounds and we can easily introduce an artificial background. Every piece of equipment from the shielding inward must be made of low-radioactivity material and/or screened for contamination.

Most of our materials are low-radioactivity by choice. One serious limitation is that we cannot use any iron near our detector package because of the presence of ${ }^{60} \mathrm{Co}$ which is used as a thickness monitor in iron smelting furnaces. We use a ${ }^{3} \mathrm{He} /{ }^{4} \mathrm{He}$ dilution refrigerator to cool our detectors to $\sim 25 \mathrm{mK}$ (see Chapter 4 ), and we cannot avoid some use of iron (particularly stainless steel). As can be seen in Figure 3.2, we isolate our commerically-purchased dilution refrigerator ${ }^{1}$ from a custom made experimental chamber, called "The Icebox." The Icebox is made primarily of high-purity copper, which can be made electrochemically to contain few radioactive isotopes.

The Icebox is connected to the dilution refrigerator via a $\sim 1 \mathrm{~m}$ copper "cold finger" at the coldest stage with thermally-isolated heatsinking layers at $50 \mathrm{mK}$, $600 \mathrm{mK}, 4 \mathrm{~K}$ (liquid helium), $77 \mathrm{~K}$ (liquid nitrogen), and room temperature as shown in Figure 3.2. The detectors are thermally isolated at the lowest temperature layer $(<50 \mathrm{mK})$ by a detector package called the "tower," shown in Figure 3.3. Each layer of the icebox is thermally connected to the corresponding layer in the tower, but these layers are isolated from each other by graphite tubes. The electrical connections are made via low-conductivity $\mathrm{NbTi}$ wires which were soldered with a custom-made ancient-lead (low-radioactivity) solder. These wires are packaged in a series of vacuum coaxes which run down one of the six hexagonal faces to each of the six detectors in the stack and are heatsunk at each thermal layer.

The SUF site has been running for several years and the cryogenic systems - often the most unreliable part of a low-temperature experiment - have been quite stable. This have proven to be an excellent site for the development and running of the low-temperature detectors described in the next chapter.

\footnotetext{
${ }^{1}$ An Oxford Instruments KelvinOx 400-S with a nominal cooling power of $400 \mu \mathrm{W}$ at $100 \mathrm{mK}$.
} 


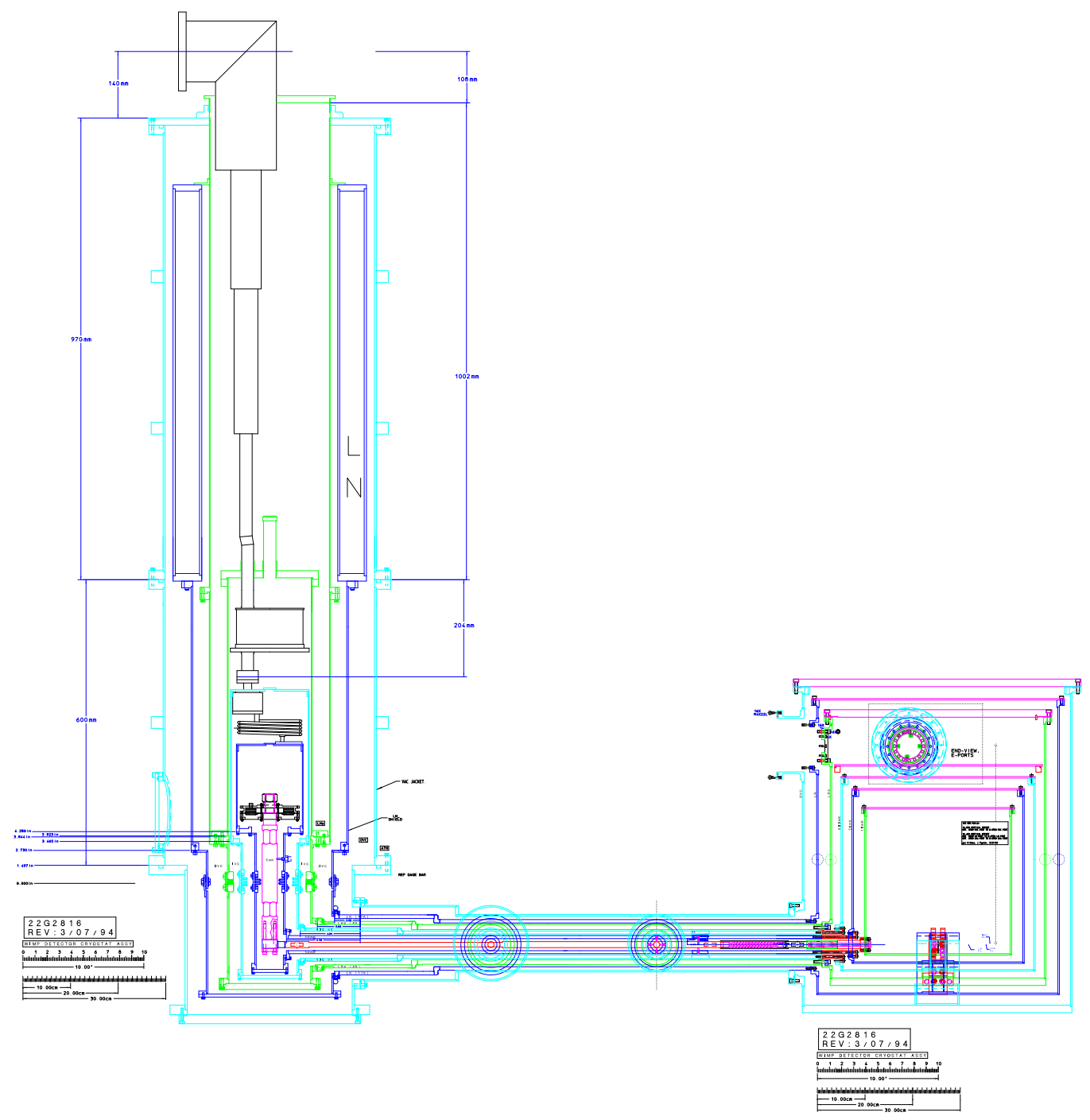

Figure 3.2: A Mechanical Drawing of the Dilution Refrigerator and the Icebox. The Icebox (right) is connected to the dilution refrigerator (left) via a $\sim 1 \mathrm{~m}$ copper "cold finger" at the coldest stage with thermally-isolated heatsinking layers at $50 \mathrm{mK}$, $600 \mathrm{mK}, 4 \mathrm{~K}$ (liquid helium), $77 \mathrm{~K}$ (liquid nitrogen), and room temperature. The detectors are thermally isolated at the lowest temperature layer $(<50 \mathrm{mK})$ by a detector package called the "Tower," shown in Figure 3.3. The signal connections are brought out to room temperature via another vacuum tube (the "e-stem") shown schematically as a series of concentric circles at the top of the Icebox. 


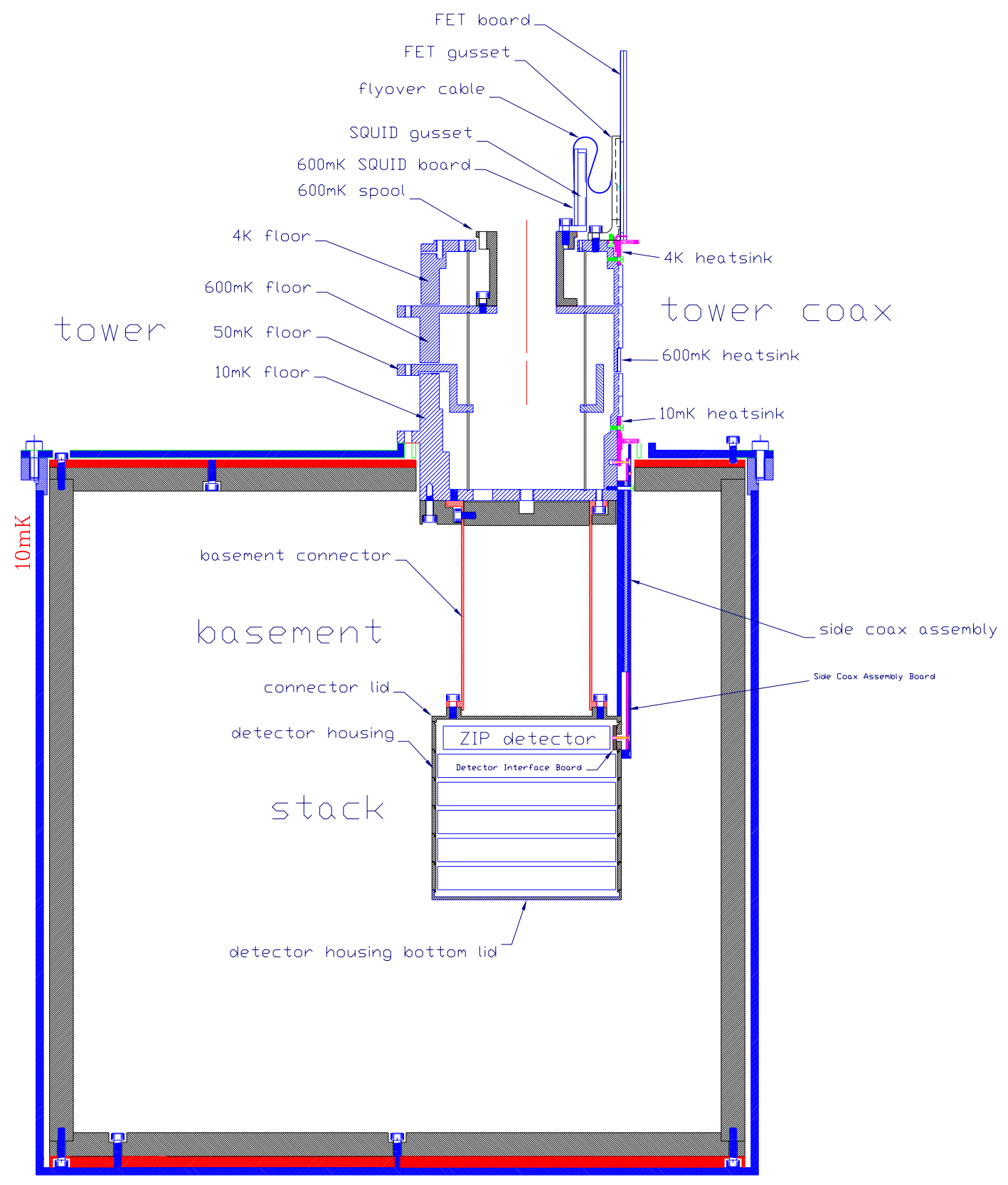

Figure 3.3: A Mechanical Drawing of the Tower. The large outer box represents the inner $(<50 \mathrm{mK})$ layer of the icebox. The tower is inserted into the icebox and makes a thermal connection to each layer of the Icebox (not shown). Each layer of the tower (labeled as "floor") is thermally isolated by graphite rods. The detector package is connected to the tower via a copper spool ("basement connector") and the electrical connections are brought down via a vacuum coax package ("side coax assembly"), shown on the right. These connections are heatsunk along with the tower to prevent thermal power from propagating from room temperature along the electronics chain. 


\section{Works Cited}

[1] D. Abrams et al. Exclusion limits on the wimp-nucleon cross section from the cryogenic dark matter search. Physical Review D, 66, 2002.

[2] A.J. da Silva. Development of a Low Background Environment for the Cryogenic Dark Matter Search. PhD thesis, The University of British Columbia, April 1997.

[3] Table of Nuclides. http://www2.bnl.gov/ton/.

[4] T.A. Perera. The Limiting Background in a Dark Matter Search at Shallow Depth. PhD thesis, Case Western Reserve University, January 2002.

[5] T. Saab. Search for Weakly Interacting Massive Particles with the Cryogenic Dark Matter Search Experiment. PhD thesis, Stanford University, August 2002.

[6] T. Saab. Measuring the abundance \& decay rate of the $10 \mathrm{kev}$ triplet. CDMS Run 21 ebook \#170, May 2002. 


\section{Chapter 4}

\section{The ZIP Detector}

CDMS ZIP detectors, shown in Figure 4.1, are 3-inch diameter, 1-cm thick disks of either silicon and germanium which are instrumented to make two complementary measurements. One face of the disk contains a thin-film superconductor which can measure an energy deposition in the crystal via the athermal phonons (crystal vibrations) produced by an interaction. Our detectors are cryogenically cooled to a temperature of $\sim 25 \mathrm{mK}$ in order to keep the noise from thermal vibrations below the threshold needed to detect the phonons from the interaction of a single particle with the crystal. As we saw in Chapter 2, having a low energy threshold is critical to determining our sensitivity to WIMPs.

The opposite side of the detector contains a simple metallic grid which is used to capacitively measure the ionization energy produced when an interaction knocks electrons loose from the lattice. The ratio of the energies from these two measurements, called the charge yield, provides a powerful background rejection parameter. Electromagnetically-interacting particles which make up our gamma and beta backgrounds produce a larger fraction of ionization energy than a neutral particle such as a neutron or a WIMP. In later chapters, we see that we can reject more than $99.9 \%$

of our gamma background and more than $95 \%$ of our beta background using this parameter.

\subsection{The Physics of an Interaction}

Before we look at how an event is actually measured, it is important to understand what happens when a particle is incident on our detectors. If the particle interacts with an electron, either through a photoelectric absorption at low energies $(\mathrm{E}<100 \mathrm{keV})$ or via a Compton scatter at higher energies, the electron is freed from the lattice if the energy deposited is greater than the binding energy $(3.8 \mathrm{eV}$ for 


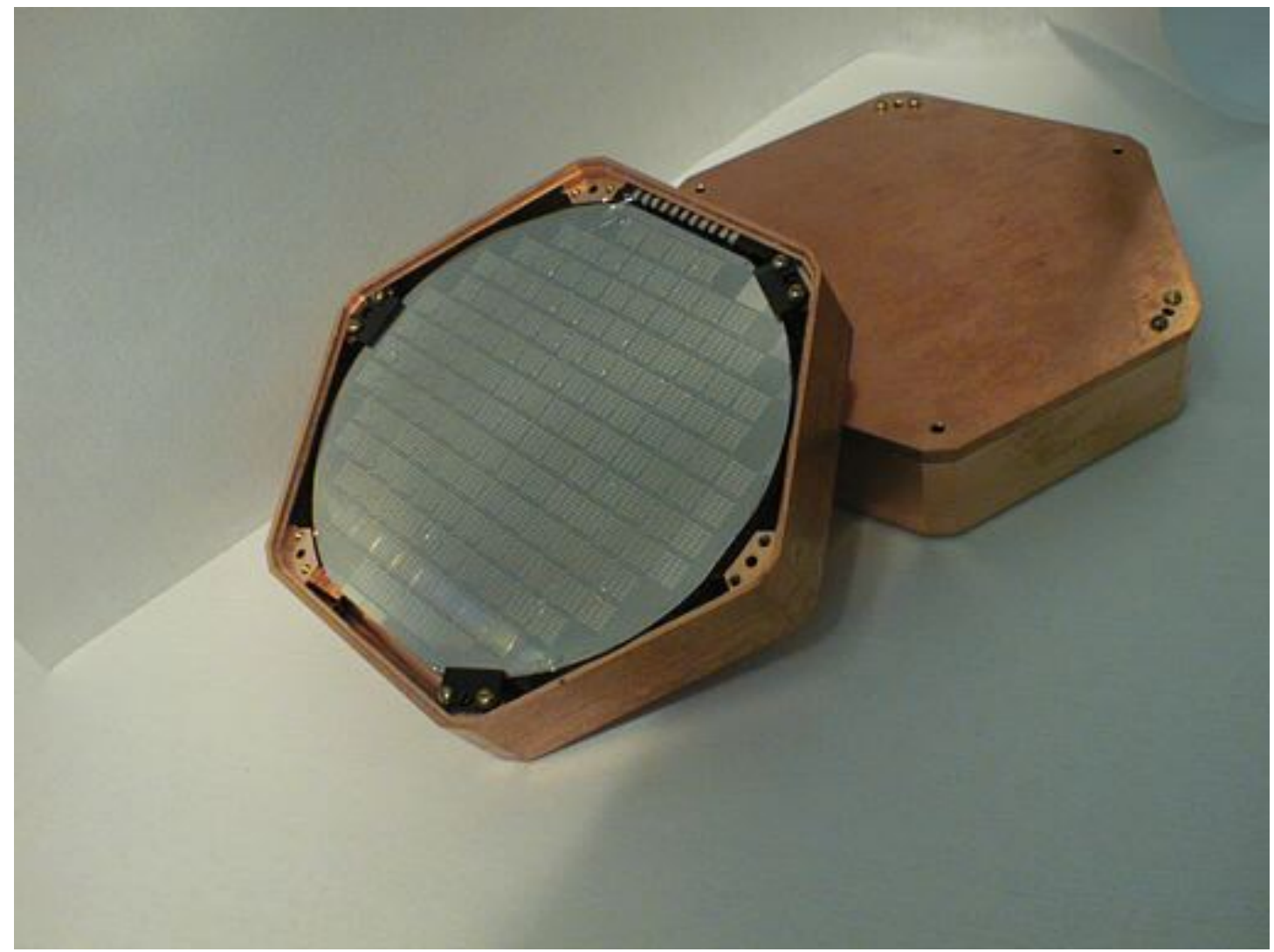

Figure 4.1: A Photograph of a ZIP detector in its Housing. The exposed face shows the phonon sensor pattern. 
Si and $3.0 \mathrm{eV}$ for Ge []$\left.^{1}\right)$. The remainder of the deposited energy is realized as kinetic energy and the electron takes a random walk (with a total path-length on the order of $35 \mu \mathrm{m}$ for a $60 \mathrm{keV}$ event in Si [2]), shedding energy by creating both electron-hole pairs as well as exciting phonons along the way. The phonons which are created by the collision have energies near the Debye frequency, typically in the $\mathrm{THz}$ range. The scattering cross-section is a steep function of frequency $\left(\sim \nu^{4}\right.$ for isotope scattering, $\sim \nu^{5}$ for anharmonic decay [2]), such that high-energy phonons quickly down-convert to lower-energy phonons until they become ballistic, that is, when their mean free path becomes of the same order as the size of the crystal $(\sim 1 T h z)$. About $30 \%$ of the deposited energy initially goes into forming electron-hole pairs this way [3] and the rest becomes phonons.

If the particle interacts with a nucleus in the crystal lattice, it causes the crystal to vibrate. In the 10-100 keV energy range that we are interested in for a WIMP scatter, the collision breaks the nucleus free from the lattice and creates a defect site $^{2}$. As much as $60 \%$ of the deposited energy initially goes into forming electronhole pairs, which is contrary to the expected result that nuclear recoils produce a smaller fraction of ionization energy than the 30\% cited above for electron recoils. The difference is in the microphysics of the nuclear recoil following the collision [3]. Rather than being deposited along an extended track, the energy is concentrated in a droplet on the order of $10 \mathrm{~nm}$ in diameter. The dominant scattering process is between the charge carriers, so the droplet remains coherent for a time on the order of 0.1 ps. Scattering processes within the droplet create additional phonons while it remains coherent, such that only $10 \%$ of the initially deposited energy remains in electron-hole pairs which can be collected by the charge channels. It is this difference in the fraction of "charge energy" $\left(E_{q}\right)$ to the fraction of "phonon energy" $\left(E_{p}\right)$ which allows us to discriminate electron recoil background events from our nuclear recoil candidate signal events. We call the ratio of these two energies the "charge yield", $y \equiv E_{q} / E_{p}$ such that the two classes of events have an approximate separation of $\frac{y_{e r}}{y_{n r}} \sim \frac{0.3 / 0.7}{0.1 / 0.9} \sim 3-4$ in this quantity.

A voltage bias across the crystal causes the electron-hole pairs to drift to opposite surfaces of the detector, where they are recombined and neutralized. As the electron-hole pairs drift, they disturb the lattice and create a second population of phonons called Neganov-Trofimov-Luke (NTL) phonons [5] [6]. This process is similar to Cherenkov radiation in that once the electron or hole has been accelerated past

\footnotetext{
${ }^{1}$ The values given in [1] are for $77 \mathrm{~K}$. Other quantities in the text suggest that these values should be acceptable at our base temperature of $\sim 25 \mathrm{mK}$. The only place that these numbers are used are in Equation 4.1

${ }^{2}$ The displacement energy is on the order of 10-20 eV for silicon[4]
} 
the speed of sound of the medium, it begins to spontaneously emit phonons. Like Cherenkov light, these phonons are focused in a forward-pointing cone. Since the NTL phonons carry away energy, they represent a kind of drag force on the traveling carriers. They quickly reach a terminal velocity where the force on the carrier due to the electric field has been balanced by the energy lost to phonon emission [2].

The work done to create NTL phonons does not come from the energy deposition, but rather from the voltage bias used to drift the charges. Since the energy which is read out by the phonon sensors is a combination of phonons created by the interaction itself as well as these NTL phonons, we must correct the measured phonon signal to account for this energy:

$$
E_{\text {phonon }}=E_{\text {recoil }}+E_{N T L}=E_{\text {recoil }}+\frac{E_{q}|V|}{E_{e-h}}
$$

where $E_{\text {phonon }}$ is the signal measured in the sensor and $E_{\text {recoil }}$ is the phonon energy deposited in the original recoil. $E_{N T L}$ is the energy of the NTL phonons created by the applied voltage, $V . E_{e-h}$ is $3.8 \mathrm{eV}$ for silicon and $3.0 \mathrm{eV}$ for germanium such that $\frac{E_{q}}{E_{e-h}}=e N_{e-h}$

\subsection{Phonon Channels}

The phonon sensors are a patterned, superconducting thin-film deposited on one of the faces of the detector. The pattern is connected such that the phonon side is divided into quadrants (labeled A-D) which form four separate phonon readout channels. Each quadrant consists of approximately 1000 tungsten meanders which are all connected in parallel. A sketch of the phonon sensor is shown in Figure 4.2 Each $250 \mu \mathrm{m} \times 1 \mu \mathrm{m} \times 35 \mathrm{~nm}$ meander has a series of aluminum fins which absorb the phonon at the crystal surface and then funnel the phonon energy into the tungsten [7.

\subsubsection{Quasiparticle Traps}

Having a thin-film sensor provides a significant improvement in detector performance relative to a purely calorimetric measurement by reducing the ratio of active sensor mass to active detector mass. We can further reduce that ratio by decreasing the amount of active sensor on the surface. This can be achieved by attaching aluminum

\footnotetext{
${ }^{3}$ There have been several iterations of the phonon sensor mask, each with some minor changes. Chapter 5 contains more details of the specific detectors used in low-background data-taking
} 

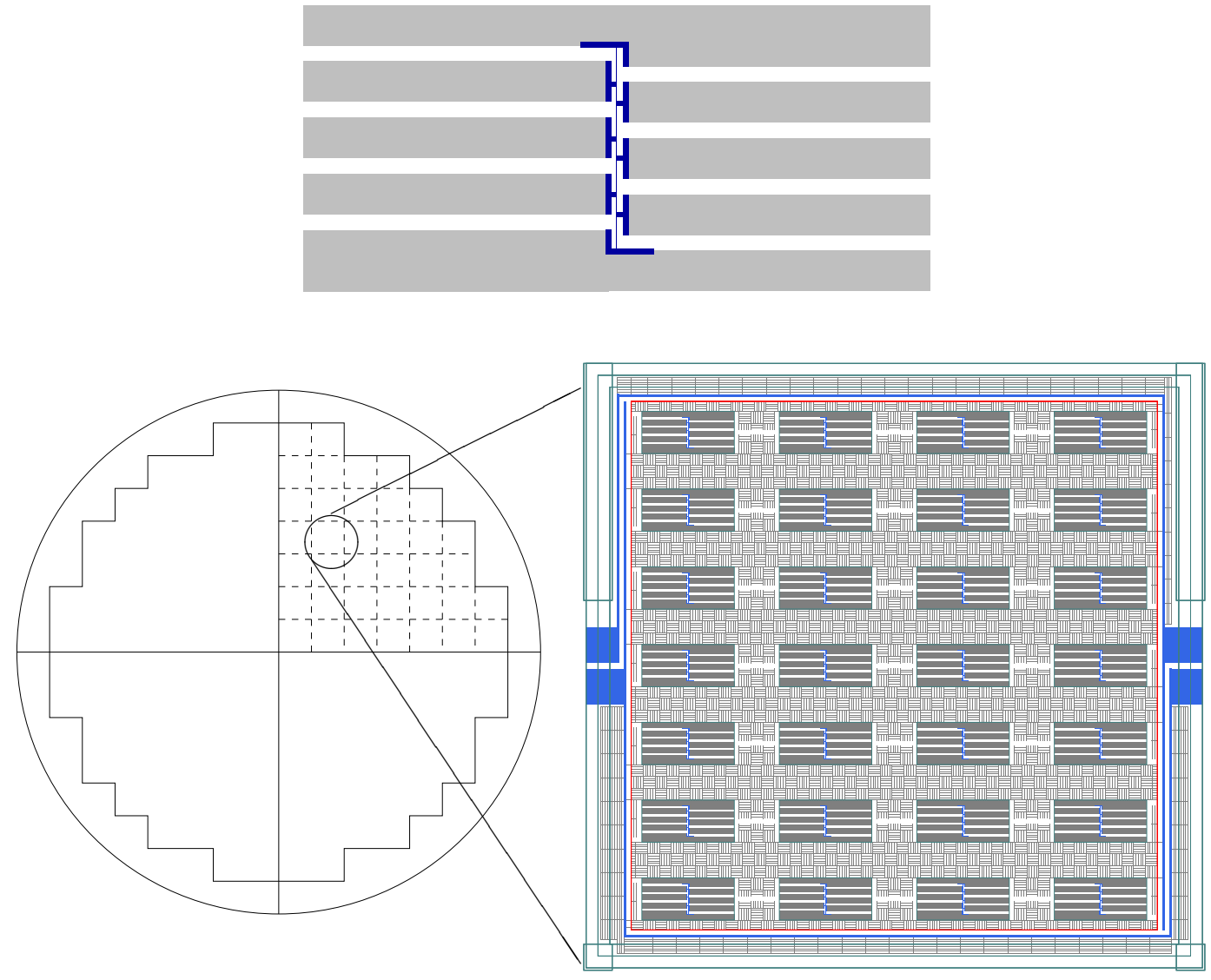

Figure 4.2: A Sketch of the ZIP Detector Phonon Sensor. The bottom left shows a schematic of one quadrant, which contains $37-5 \mathrm{~mm} \times 5 \mathrm{~mm}$ square dies. The bottom right shows a magnified view of one of those dies, each of which contains $4 \times 7=28$ sensors. At the top is a drawing of an isolated sensor, with the tungsten meander shown in dark blue and the aluminum quasiparticle traps shown in light grey. The pattern shown is the one used for the Run 21 germanium detectors. 

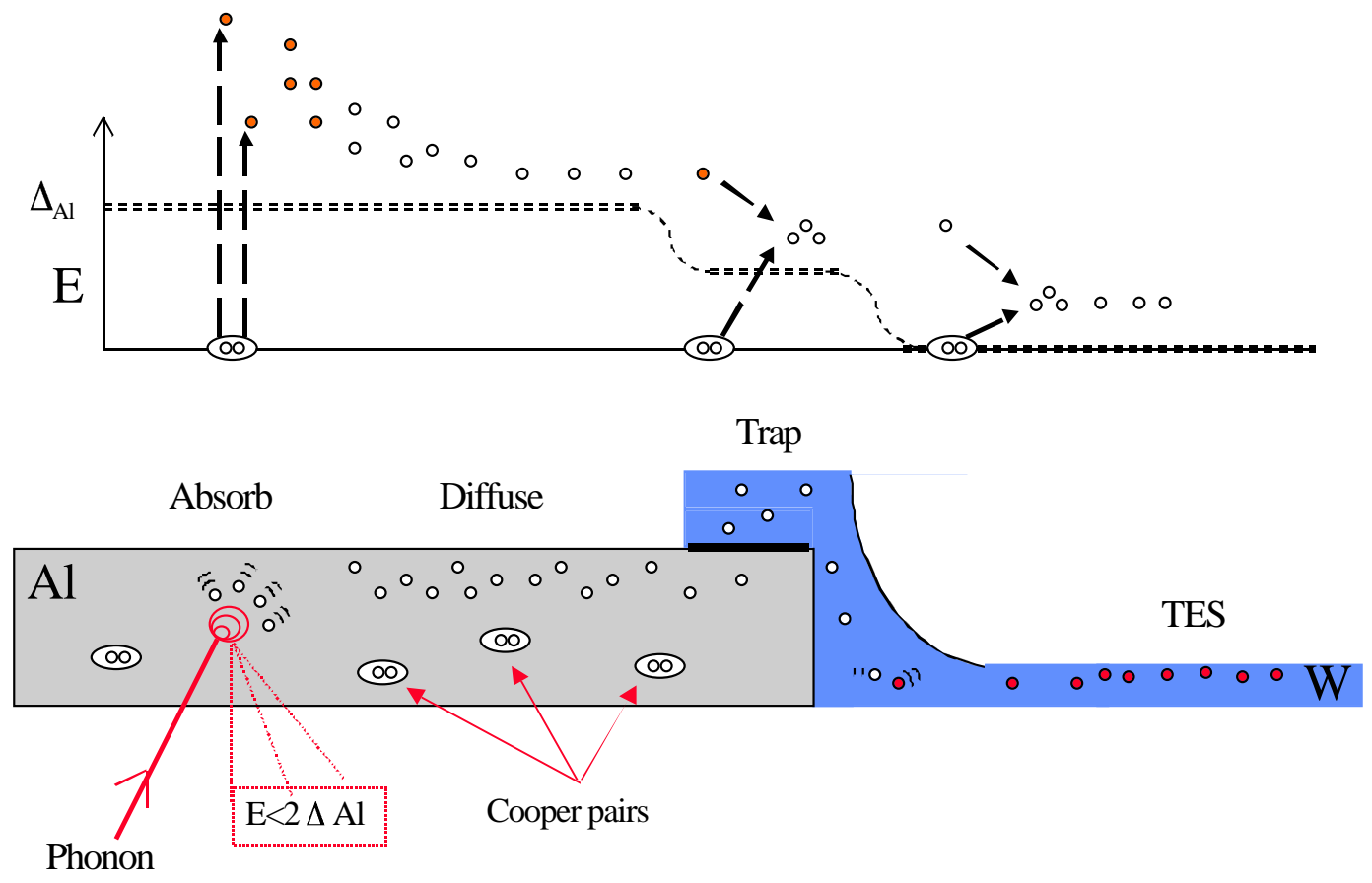

Figure 4.3: Superconducting Gap Structure of the Quasiparticle Traps. At the bottom is a sketch of the sensor, with a representation of a scattering event. A phonon incident on the aluminum fins break cooper pairs and create quasiparticles. As shown in the sketch of the band structure at the top, the tungsten has a lower superconducting gap than the aluminum and the quasiparticles diffuse towards the meander. Once inside the tungsten, they scatter into lower-energy quasiparticles and are trapped in the tungsten. Finally, the quasiparticles thermalize and increase the temperature of the meander.

fins to our tungsten sensors. Aluminum has a higher $T_{c}$ and a higher superconducting gap energy than tungsten (see Figure 4.3). A phonon which is incident on the aluminum can break Cooper pairs if the energy is greater than the pair-binding energy, $2 \Delta_{A l}=0.358 \mathrm{meV}[8]$. This creates quasiparticles - perturbations in the overlapping wavefunction of the atoms in the aluminum. These quasiparticles diffuse towards the tungsten, where they deposit their energy due to the lower superconducting gap (see Figure 4.3).

The efficiency of this diffusion is critically dependent on the geometry of the traps. Ballistic phonons in the substrate have an energy of $E \sim h \nu \sim\left(4 \times 10^{-15} \mathrm{eV} \mathrm{s}\right)(1 \mathrm{THz}) \sim$ $4 \mathrm{meV}>20 \Delta_{A l}$, so a phonon incident on the aluminum creates an initial pair of quasiparticles which then cascades into a number of quasiparticles with characteris- 
tic energies near the pair-binding energy. Scattering processes during diffusion can also create lower-energy phonons, many of which are below the gap energy and are permanently lost back to the substrate. The cross-section of the film determines the mean free path of the quasiparticles, while the length of the fins relative to the mean free path determines how much of the energy is lost back to the substrate.

The design of the sensor geometry must balance two effects. First, the fins should have a large total surface area so that phonons can be efficiently absorbed into the aluminum. At the same time, the fins must not be much longer than a few mean free path lengths or too much of the absorbed energy will be lost back into the substrate before being absorbed into the tungsten. We also have two constraints from processing. The aluminum fins cannot be too thick or they cannot be processed to overlap with the tungsten [9]. We are also limited to a maximum of about 1000 meanders per quadrant (given our current meander geometry) due to noise considerations and to prevent $\mathrm{L} / \mathrm{R}$ oscillations with the readout electronics [10].

Our current design, shown in Figure 4.2, uses 10 - $300 \mathrm{~nm}$ thick fins per meander which are $380 \mu \mathrm{m}$ long and $60 \mu \mathrm{m}$ wide. The expected quasiparticle collection efficiency for this design is $24 \%[9]$. This configuration has only a $\sim 5 \%$ surface area coverage, but previous experiments have shown that the phonons lose little energy upon reflections with the bare crystal surface and bounce around with little loss until they are absorbed at a metal surface. There is a comparable amount of metalized surface in the ionization sensor and the readout lines, so we would expect a maximum phonon collection efficiency to be on the order of $10 \%$ (for phonons with $E>2 \Delta_{A l}$ ).

\subsubsection{Electrothermal Feedback}

Each of the quadrants can be treated as a Transition-Edge Sensor with an equivalent

resistance of $R \approx \frac{R_{\text {meander }}}{N} \sim 1 \Omega$. A sample resistance-vs.-temperature curve for a quadrant is shown in Figure 4.4. We attain the maximum sensitivity within the superconducting transition, that is, we measure the largest change in resistance $(\Delta R)$ for the smallest change in temperature $(\Delta T)$. We can express this sensitivity as:

$$
\alpha \equiv \frac{d R}{R} / \frac{d T}{T}
$$

The sensor can be biased into the transition through Joule heating with a constant voltage $\left(P=V^{2} / R\right)$. We saw above that in the transition, small changes in temperature produce large changes in resistance. If we then examine the corresponding change in Joule heating (keeping the voltage fixed) we have:

$$
\frac{d P}{d T}=\frac{d P}{d R} \frac{d R}{d T}=-\frac{V^{2}}{R^{2}} \frac{d R}{d T}=-\frac{P_{0}}{T} \frac{d R}{R} / \frac{d T}{T}=-\frac{P_{0} \alpha}{T}
$$




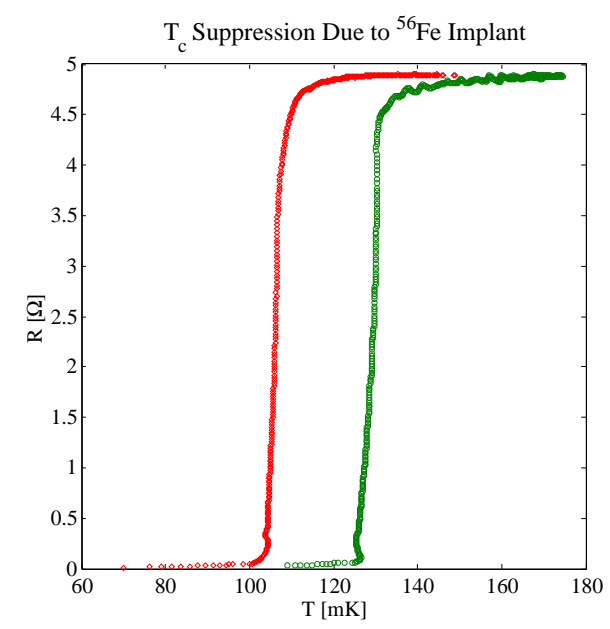

Figure 4.4: Typical resistance-vs.-temperature curve of a Phonon Sensor. Two curves are shown for the same quadrant, one before (green, left) and one after (red, right) iron implantation, described in Chapter 5. The iron implantation process lowers the $T_{c}$ of the sensor into the desired range without altering the shape of the transition curve.

For small perturbations in temperature, negative feedback forces the sensor to stay at our chosen bias point. The strength of that feedback depends on both $\alpha$ and $T$.

A more careful examination of the thermal properties of the circuit yields an additional benefit of this feedback [11]. In equilibrium, the Joule heating keeps the circuit above the substrate temperature $\left(T_{s}\right)$ since:

$$
C \frac{d T}{d t}=\frac{V^{2}}{R(T)}-K\left(T^{n}-T_{s}^{n}\right)=0
$$

where $\mathrm{K}$ is a geometry- and material-dependent quantity. The index $n$ depends on the dominant thermal impedance between the sensor and the substrate. For thin films at low temperatures, the electronic and phonon systems are decoupled and $n=5$. Small perturbations in the temperature, $\Delta T$, cause small deviations in resistance. Expanding our power flow equation to first order, we have

$$
C \frac{d \Delta T}{d t}=-\frac{V^{2}}{R_{0}^{2}} \frac{d R}{d T} \Delta T-g \Delta T=-\frac{P_{0} \alpha}{T_{0}} \Delta T-g \Delta T
$$

where $g=d P / d T=n K T^{n-1}$ and we have substituted the equilibrium value $P_{0}=$ $V^{2} / R_{0}$. The solution to this differential equation is a simple exponential with time 
constant

$$
\tau_{\text {eff }}=\frac{\tau_{0}}{1+\frac{\alpha}{n}\left(1-\frac{T_{s}^{n}}{T_{0}^{n}}\right)}
$$

where $\tau_{0}=C / g$ is the time constant without feedback. If our transition temperature is sufficiently higher than the substrate temperature $\left(T_{0}^{n} \gg T_{s}^{n}\right)$ and we have a sharp enough transition $(\alpha \gg n)$, this simplifies to

$$
\tau_{e f f} \approx \frac{\tau_{0} n}{\alpha}
$$

In this extreme electrothermal feedback regime, the electronic circuit can now respond to changes in temperature faster than the energy can be lost to the substrate. When a pulse deposits energy into the tungsten meanders, the power supply provides less Joule heating and we can then read out the energy deposited in the sensor directly by monitoring the current into the voltage-biased circuit:

$$
E=\int P d t=V_{0} \int \Delta I d t
$$

\subsection{Charge Channels}

The charge channels are a straightforward capacitive circuit which is instrumented in two segments, an inner disk and an outer ring. The inner disk covers approximately $85 \%$ of the surface of the detector. There is a small $1 \mathrm{~mm}$ gap and the outer ring is about $3 \mathrm{~mm}$ thick. The outer ring acts like a guard ring since irregularities in the electric field due to edge effects may effect our charge collection. This creates a protected inner fiducial volume which is shielded from background events coming in through the side of the detector. The disk and ring surfaces are covered by a grid of $2 \mu \mathrm{m}$ aluminum lines spaced at $20 \mu \mathrm{m}$ intervals. The phonon circuit also contains a similar grid between the fin and meander structures. Both this grid and the phonon sensors themselves act as a virtual ground for the charge circuit.

A bias of several volts across the $1 \mathrm{~cm}$-thick crystal causes charge-hole pairs created from a collision to drift to opposite surfaces. Events which occur near the surface can lose some of their charge carriers via back-diffusion into the similarly-charged contact. This causes the measured ionization energy to be lowered and the separation of background and signal events in our discrimination parameter, the "yield" (the ratio of the charge energy to the phonon energy), to be decreased.

This "dead layer" can be reduced by the addition of a thin layer of amorphous 
silicon ${ }^{4}$ sputtered on the bare substrate (either silicon or germanium) before the deposition of the tungsten and aluminum on either side of the detector. Amorphous silicon has a larger bandgap $(1.2 \mathrm{eV})$ than either germanium $(0.74 \mathrm{eV})$ or silicon $(1.17 \mathrm{eV})$, so it creates a potential barrier between the substrate and the charge electrodes. Back-diffusion is more difficult on the similarly-charged side, while the applied bias voltage is more than sufficient to overcome the barrier at the oppositelycharged side.

${ }^{4}$ The Run 21 silicon detectors have a 21-nm layer of amorphous silicon. The germanium detectors were made later and have a 40-nm thick layer. 


\section{Works Cited}

[1] G.F. Knoll. Radiation Detection and Measurement. John Wiley and Sons, Inc., 1999.

[2] B. Cabrera et al. Prompt phonon signals from particle interactions in si crystals. In LTD-5 [12], pages 365-375.

[3] S.E. Esipov et al. Ballistic phonon emission from electron-hole droplets: Application to the nuclear recoil problem. In LTD-5 [12], pages 377-385.

[4] H.J. Maris and S. Tamura. Anharmonic decay and the propagation of phonons in an isotopically pure crystal at low temperatures: Application to dark-matter detection. Physical Review B, 47(2):727-739, January 1993.

[5] B. Neganov and V. Trofimov. USSR patent no. 1037771. 1985.

[6] P.N. Luke. Journal of Applied Physics, 64:6858, 1988.

[7] R.M. Clarke. An Athermal Phonon Mediated Dark Matter Detector with Surface Event Discrimination. PhD thesis, Stanford University, May 1999.

[8] C.P. Poole, Jr., editor. Handbook of Superconductivity. Academic Press, 2000.

[9] T. Saab. Search for Weakly Interacting Massive Particles with the Cryogenic Dark Matter Search Experiment. PhD thesis, Stanford University, August 2002.

[10] Sae Woo Nam. Development of Phonon-Mediated Cryogenic Particle Detectors with Electron and Nuclear Recoil Discrimination. PhD thesis, Stanford University, December 1998.

[11] K.D. Irwin. Phonon-Mediated Particle Detection Using Superconducting Tungsten Transition-Edge Sensors. PhD thesis, Stanford University, February 1995.

[12] Fifth International Workshop on Low Temperature Detectors, volume 93. Plenum Press, November 1993. 


\section{Chapter 5}

\section{Development of CDMS II Detectors}

The previous chapter dealt with the properties of an idealized CDMS detector, but in the real world it is impossible to achieve ideal conditions. Detector processing is a very critical step and we have implemented a rigorous testing program to assure quality control. The ultimate goal is to provide our experiment with a total of 30 detectors which meet our specifications for a dark matter search. The six Run 21 detectors whose results are described in later chapters are the first installment of those 30 detectors which will eventually be installed in the CDMS II (deep underground) phase of the experiment.

The six detectors in "Tower 1" are labeled by their position in the stack, from Z1 to Z6 (the "Z" denotes that this is a "ZIP" model detector). Prior to their installation they were referred to by different names, such as "G11" or "S3," where the initial letter represents the material (either Germanium or Silicon) and the number is a production series number. Table 5.1 lists both names for a detector, since they are identified differently depending on whether we are discussing the production stage or the data-taking stage.

\subsection{Detector Uniformity}

The phonon sensor is comprised of many individual meanders which have slightly different properties. Our goal is to produce detectors which have the $T_{c}$ of all their meanders within the approximate range $60 \mathrm{mK}-90 \mathrm{mK}$. The lower limit comes from the electrothermal feedback requirement that $T_{c} \gg T_{\text {substrate }}$ (see Section 4.2.2). The upper limit comes from the length of our tungsten meanders $(250 \mu \mathrm{m})$. Above $90 \mathrm{mK}$, the $\sim 4 \mathrm{meV}$ ballistic phonons can drive part of the meander normal while 


\begin{tabular}{|c|c|}
\hline Stack Name & Production Name \\
\hline Z1 & G6 \\
\hline Z2 & G11 \\
\hline Z3 & G8 \\
\hline Z4 & S3 \\
\hline Z5 & G9 \\
\hline Z6 & S1 \\
\hline
\end{tabular}

Table 5.1: Detector names for CDMS Tower 1. The "stack name" indicates its position in the detector package while the "production name" refers to the name it was give prior to installation, where " $G$ " detectors are germanium and " $\mathrm{S}$ " detectors are silicon.

the rest of the meander remains superconducting [1] 2]. The net result is to widen the transition and decrease the sensitivity, $\alpha$ (Equation 4.2), since the resistance of the meander is then proportional to the fraction of the meander which is normal.

Our films have individual meanders with widths on the order of $\Delta T \sim 1 \mathrm{mK}$, but a $T_{c}$ gradient across the surface of the detector produces a width of $\Delta T \sim 20 \mathrm{mK}$ across a quadrant. We can tolerate some variation in $T_{c}$ across the surface since electrothermal feedback will still bias an individual meander into the transition region if it is within a few $\mathrm{mK}$ of the transition[1. Practically, though, having a $T_{c}$ gradient in our phonon sensor results in a position dependence in our sensitivity. This can be corrected for offline, but at the expense of the extra work of calibrating the energy response as a function of position and producing a correction matrix.

When tungsten is deposited as a thin film, its superconducting transition temperature is significantly higher than the $15 \mathrm{mK}$ quoted for tungsten in a bulk sample due to the presence of different phases in the tungsten lattice. Our processing typically produces detectors with $T_{c} s$ in the range $100-200 \mathrm{mK}$, well above our target range. We do not have sufficient control over the $T_{c}$ of the tungsten as it is deposited, but implanting the sensors with a small amount of magnetic material such as iron can lower the $T_{c}$ into the desired range in a controlled fashion[3]. Detector characterization is critical in determining the needed amount of implantation. We use the data taken from three separate measurements prior to implantation to model the variation of $T_{c}$ across the detector 4]. This information is then used to devise a masking pattern for a multi-stage implantation which not only brings the phonon sensor into the desired range, but also makes the $T_{c}$ distribution more uniform.

After implantation, we perform the same tests as before in order to verify that the iron implantation went well and that the detector meets the specifications necessary for running in the experiment. Our implantation scheme is necessarily conservative and in some cases a detector may require a second implantation. Our ability to 
fine-tune the phonon sensor through repeated implantation is limited since we can over-implant the sensors below the range of useful $T_{c}$ and there is no proven way to remove the implantation once it has been done.

\subsection{Normal and Parasitic Resistance}

Each quadrant of the phonon sensor is composed of $37-5 \mathrm{~cm} \times 5 \mathrm{~cm}$ blocks of 28 tungsten meanders each for a total of 1036 meanders per quadrant which are all connected in paralle 1 . Each meander is a piece of tungsten $35 \mathrm{~nm} \times 2 \mu \mathrm{m} \times 200 \mu \mathrm{m}$ in dimension. A $35 \mathrm{~nm}$ thick tungsten film has an approximate normal-state resistivity of $5 \Omega$ per square, which gives a resistance of $R=\frac{R_{m}}{N} \sim 1 \Omega$.

A first quick check of sensor quality is to measure the normal and parasitic resistances. When the meander is superconducting, a parasitic resistance in the circuit remains which is typically on the order of $10 \mathrm{~m} \Omega$. A high value for parasitic resistance indicates an obvious problem with the circuit, whether in the sensor itself or somewhere in the electronics. The normal resistance is measured by heating the refrigerator base layer to a temperature of $\sim 250 \mathrm{mK}$, well above our tungsten transition temperature but below the transition of the aluminum leads. Since the value of $5 \Omega$ per square is dependent on the thickness of the film, the actual value of the normal resistance can give us a crude diagnostic of the deposition.

A short circuit anywhere in the quadrant is disastrous since a short in parallel shorts out the whole circuit. A high value for the normal resistance of a quadrant can indicate a break in the aluminum rails connecting the sensors since it causes large sections of the circuit to be disconnected. The sensor can still work with broken meanders, but these lead to dead spots on the detector. Both of these failure modes can theoretically be repaired by the fabrication team, but this requires that the detector be removed from the fridge, returned for repair, and then retested, a process which can take several weeks. Table 5.2 shows a sample of these values for one of the Run 21 detectors (Z5/G9).

\subsection{Resistance vs. Temperature}

Simply measuring the resistance of the sensor while sweeping the temperature of the detector, while useful, is not sufficient to fully characterize our sensor. The current used to make the resistance measurement has a free path through a quadrant even

\footnotetext{
${ }^{1}$ The two silicon detectors, S1 and S3, are based on an older deposition mask and have $37 \times 24=$ 888 meanders per quadrant
} 


\begin{tabular}{|c|c|c|c|c|c|c|}
\hline \multirow{2}{*}{$\begin{array}{l}\text { Z5 } \\
\text { Channel }\end{array}$} & \multicolumn{3}{|l|}{ Pre-implantation } & \multicolumn{3}{|c|}{ Post-implantation } \\
\cline { 2 - 7 } & $R_{\text {para }}$ & $R_{\text {norm }}$ & $T_{\text {trans }}$ & $R_{\text {para }}$ & $R_{\text {norm }}$ & $T_{\text {trans }}$ \\
\hline $\mathrm{A}$ & $9.1 \mathrm{~m} \Omega$ & $1.55 \Omega$ & $123-127 \mathrm{mK}$ & $12.1 \mathrm{~m} \Omega$ & $1.48 \Omega$ & $79-85 \mathrm{mK}$ \\
\hline $\mathrm{B}$ & $9.5 \mathrm{~m} \Omega$ & $1.66 \Omega$ & $129-139 \mathrm{mK}$ & $10.9 \mathrm{~m} \Omega$ & $2.17 \Omega$ & $83-87 \mathrm{mK}$ \\
\hline $\mathrm{C}$ & $11.2 \mathrm{~m} \Omega$ & $1.85 \Omega$ & $112-116 \mathrm{mK}$ & $16.5 \mathrm{~m} \Omega$ & $2.17 \Omega$ & $81-85 \mathrm{mK}$ \\
\hline $\mathrm{D}$ & $13.0 \mathrm{~m} \Omega$ & $2.00 \Omega$ & $121-126 \mathrm{mK}$ & $21.1 \mathrm{~m} \Omega$ & $1.53 \Omega$ & $82-99 \mathrm{mK}$ \\
\hline
\end{tabular}

Table 5.2: Basic Phonon Sensor Properties for Detector Z5 (G9). Data are given for each of the four phonon channels (quadrants A-D). Shown are the normal and parasitic resistances, along with the $T_{c}$ range derived by a resistance-vs.-temperature sweep (Section 5.3). Values are shown both prior to and after the iron implantation (see text).

if only one of the meanders is superconducting since the meanders are connected in parallel. This means that a resistance-versus-temperature curve is only sensitive to the upper end of the transition curve, that is, we are only measuring the transition of the highest- $T_{c}$ meanders.

Figure 5.1 shows a simplified circuit diagram of a ZIP phonon sensor. We monitor the resistance of a quadrant $\left(R_{T E S}\right)$, by applying a small triangle wave $\left(I_{\text {bias }} \sim 3 \mu \mathrm{A}\right.$ peak-to-peak at $100 \mathrm{~Hz}$ ) and measuring the current through the sensor $I_{T E S}$, by observing the voltage on $R_{\text {feedback }}$ as inductively coupled through the SQUID array [5]. We start at a temperature at which the quadrant is superconducting and increase the temperature of the TES by applying heat to the fridge. Operationally, it is important to use as small a current as possible to measure the resistance. The phase space for a superconducting transition is a function of three quantities: temperature, current, and magnetic field strength. We are interested in the temperature-dependent behavior, so we want to minimize the current and shield our apparatus against magnetic fields.

Table 5.2 lists two numbers for the transition temperature. The first number represents the temperature at which the triangle wave output starts to differ from the superconducting value. This does not happen at the temperature of the lowest $T_{c}$ meanders, but rather when the input triangle wave starts to drive normal the few remaining sensors at the top of the curve. The second number is the point at which the triangle wave output reaches its normal value.

Figure 5.2 shows the approximate area of the sensor this measurement maps out given a $T_{c}$ gradient across the sensor. The $T_{c} s$ of the resistance-vs.-temperature sweep give the general direction of the gradient. These four initial data points are the starting point for a more detailed study of the $T_{c}$ distribution detailed below. 


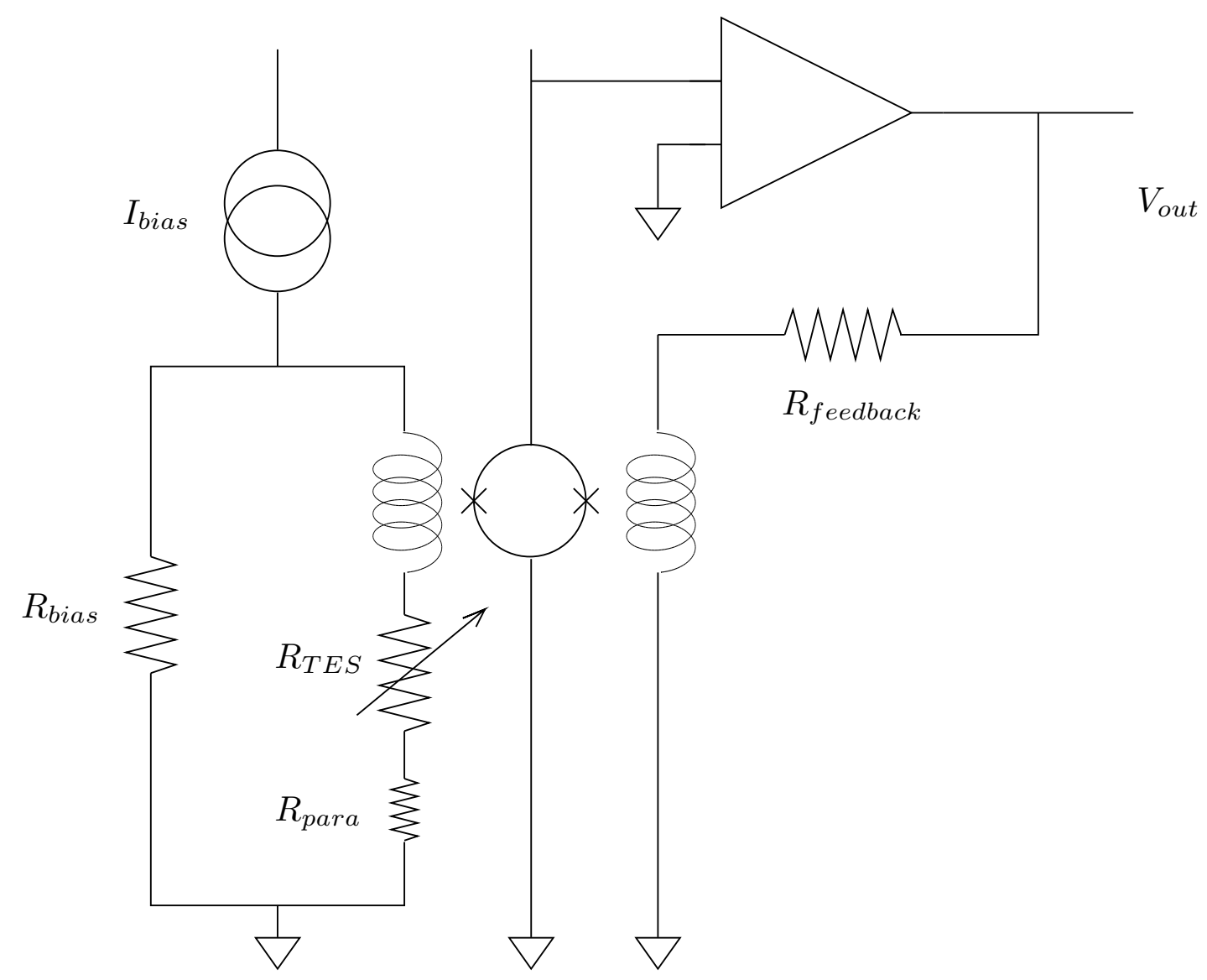

Figure 5.1: Simplified Circuit Diagram for the Phonon Sensor. The sensor $\left(R_{T E S}\right)$ is voltage biased by applying a DC current in parallel with a shunt resistor $\left(R_{\text {bias }}=\right.$ $20 \mathrm{~m} \Omega$ ). We measure the current in the sensor via inductive coupling to a DCbiased SQUID array, which is amplified via a feedback loop (which is shown here schematically as a single amplifier) and read out at $V_{\text {out }}$. 


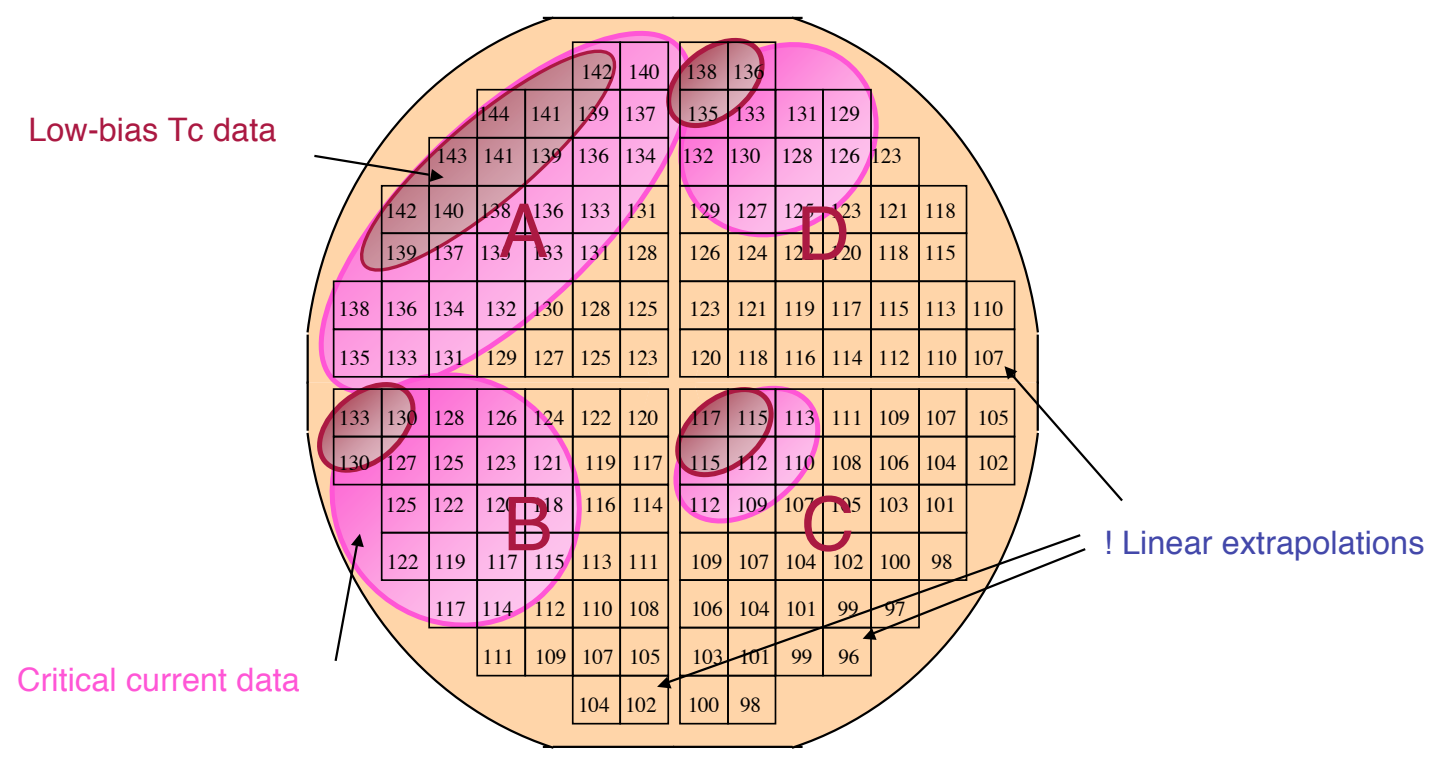

Figure 5.2: Geometrical Sensitivity to $T_{c}$ of Resistance-vs.-Temperature and Critical Current Measurements. Separate characterizations are done for each of the four quadrants, labeled A-D. The numbers show the modeled values for the $T_{c}$ of each $5 \mathrm{~mm}$ square die (in $\mathrm{mK}$ ). The shaded areas indicate the approximate regions that the measurements in each quadrant would be sensitive to based on the proposed $T_{c}$ gradient. The resistance-vs.-temperature ("Low-bias $T_{c}$ " Section 5.3) measurement is sensitive to the meanders with the very highest $T_{c} s$ and gives us four anchor points with which to determine the overall direction of the gradient. The critical current (Section 5.4) measurement covers roughly half of the surface area, corresponding to $T_{c} s$ in the upper half of the transition curve for each quadrant. The lower half of the transition curve is a linear extrapolation. The modeled gradient is verified via the IbIs measurement (Section 5.5) which is sensitive to the whole transition curve, including the lowest $T_{c} s$. 


\subsection{Critical Current}

The graph of resistance as a function of temperature as measured above is essentially a measurement of the critical current of the highest $T_{c}$ meanders since the $\sim 1 \mathrm{mK}$ width of a single meander is much smaller than the $\sim 10 \mathrm{mK}$ width across a quadrant. A more complete measurement can be made by measuring the critical current of the entire quadrant while sweeping the temperature. Assuming that the meanders have similar critical currents, the critical current of the whole quadrant essentially measures the number of meanders which are superconducting at a given temperature.

A simplified description of the situation can be made by assuming that all of the meanders are identical except that there are two different values of $T_{c}, T_{c 1}$ and $T_{c 2}$. When the temperature is between the two $T_{c} s\left(T_{c 1}<T<T_{c 2}\right)$, the $T_{c 1}$ meanders are normal and the $T_{c 2}$ meanders are superconducting. As we increase the current into the quadrant, the current is equally divided among the superconducting $T_{c 2}$ meanders. At some point, the circuit becomes completely normal and the total current is just the critical current of a single meander multiplied by the number which were superconducting at zero current.

The real situation is more complicated since every meander has a slightly different $T_{c}$ and critical current, but we can estimate how many meanders are superconducting at a given temperature by making a measurement of the critical current. The resistance vs. temperature curves for the four quadrants indicate the direction of the gradient across the whole wafer. We can then use the critical current data to model $T_{c}$ as a function of position and use that to make a template for iron implantation. Figure 5.2 shows the extended area of the sensor which is mapped by this measure-

ment. The critical current measurements are not very sensitive to the bottom half of a gradient since more meanders are sharing the current at low temperatures and the variations in $T_{c}$ and critical current dominate the uncertainty of the measurement. The values of the critical current are also higher and the precision of the measurement relative to the critical current of a single meander decreases.

Sample critical current curves both before and after implantation are shown in Figure 5.3, where the iron implantation has successfully brought the four quadrants into better agreement.

\subsection{IbIs - Sensor Current vs. Bias Current}

The "IbIs" measurement was developed as another means of measuring the $T_{c}$ gradient of a detector [6]. It is a slow scan of bias current $\left(I_{b}\right)$ which outputs the current passing through the sensor $\left(I_{s}\right)$. Joule heating takes it through three regions of behavior: when the quadrant is completely superconducting, when it is completely normal, and 

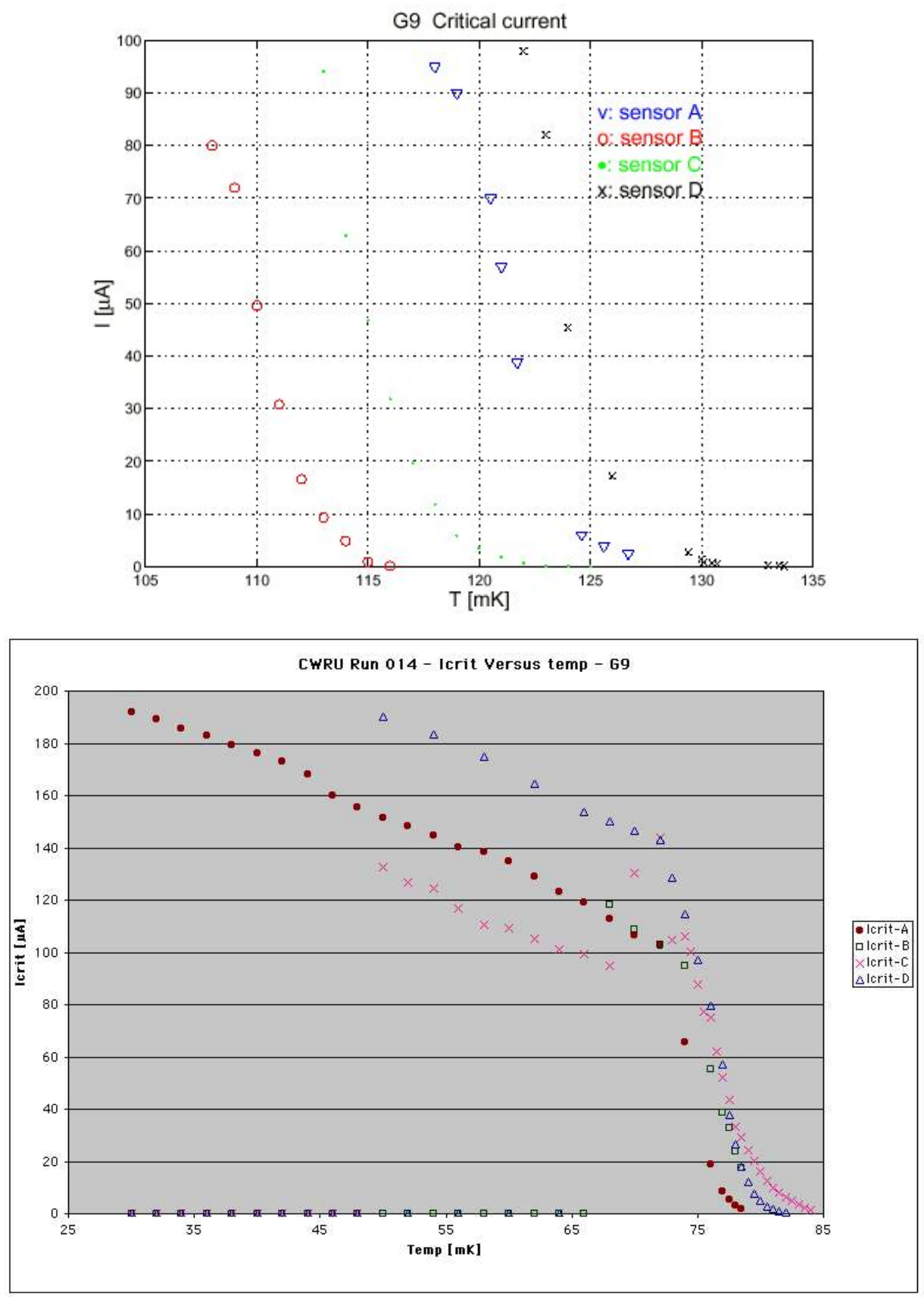

Figure 5.3: Critical Current Curves for Detector Z5 (G9). The top graph shows the critical current curves for G9 before implantation. A $T_{c}$ gradient is indicated as the four quadrants (Sensors A-D) have curves which are separated by $\sim 15 \mathrm{mK}$. After implantation (bottom), the curves are much more uniform and have a gradient of less than $10 \mathrm{mK}$. 
a transition region.

The slope of the IbIs curve $\left(I_{s} v s . I_{b}\right)$ is is a function of the resistance of the sensor (see Figure 5.1):

$$
\begin{gathered}
I_{s}=\frac{V}{R_{s}}=\frac{I_{\text {shunt }} R_{\text {shunt }}}{R_{s}}=\frac{\left(I_{b}-I_{s}\right) R_{\text {shunt }}}{R_{s}} \\
\rightarrow R_{s}=\left(\frac{I_{b}}{I_{s}}-1\right) R_{\text {shunt }} \rightarrow I_{s}=\frac{I_{b}}{\left(\frac{R_{s}}{R_{\text {shunt }}}+1\right)}
\end{gathered}
$$

For most of the sweep, the sensor is either totally normal $\left(R_{s} \gg R_{\text {shunt }} \rightarrow I_{s} \approx\right.$ $\frac{I_{b} R_{\text {shunt }}}{R_{n}}$ ) or totally superconducting (parasitic resistance gives us $\frac{R_{s}}{R_{\text {shunt }}} \sim 1 / 2 \rightarrow I_{s} \approx$ $\left.\frac{2}{3} I_{b}\right)$.

A sample output from the IbIs routine is shown in Figure 5.4. From the above relations, we can make a curve of $R$ vs. $I_{b}$ as well as $P$ vs. $I_{b}$. The lighter lines on each curve represent a linear fit to the normal part of the curve, which is flat on the plot of resistance and parabolic on the plot of power $\left(P=I^{2} R\right)$ as expected. Since we have a simple way of measuring the normal and parasitic resistances, we are primarily interested in the region where $R_{s}$ is changing. The power flow into the substrate is proportional to $T^{5}$ (see Equation 4.4 ), such that the power in the transition region is approximately constant for sufficiently narrow transition widths and hence $R(I)=\frac{P}{I^{2}}$. The slope of the power curve in the transition region is then sensitive to the width of the transition. Slightly rewriting the balanced power flow equation:

$$
I_{b}^{2} R\left(I_{s}\right)=K\left(T^{n}-T_{s}^{n}\right)
$$

we can reconstruct an $R(T)$ curve. We make a very slow sweep of $I_{b}$ so that the power flow can remain balanced. We are now also sensitive to the lower end of the transition because we are allowing the electrothermal feedback (Section 4.2.2) to bias the sensor into the transition region.

Based on these three measurements, resistance-vs.-temperature, critical current, and IbIs, we can produce a implantation scheme which will both bring our sensor into the range of acceptable $T_{c}$ and make it more uniform. The planned $T_{c}$ mask for detector Z5 (G9) is shown in Figure 5.5. The data in Table 5.2 indicate that we have reduced the $T_{c}$ into our approximate range and the critical current data shown in Figure 5.3 shows that the $T_{c}$ for the four quadrants is now in better agreement.

We have seen that detector processing, combined with a rigorous testing plan and an iron implantation, can produce detectors within the specifications for a dark matter search. The six detectors used in the experimental running described in the following chapters were the result of this process. 

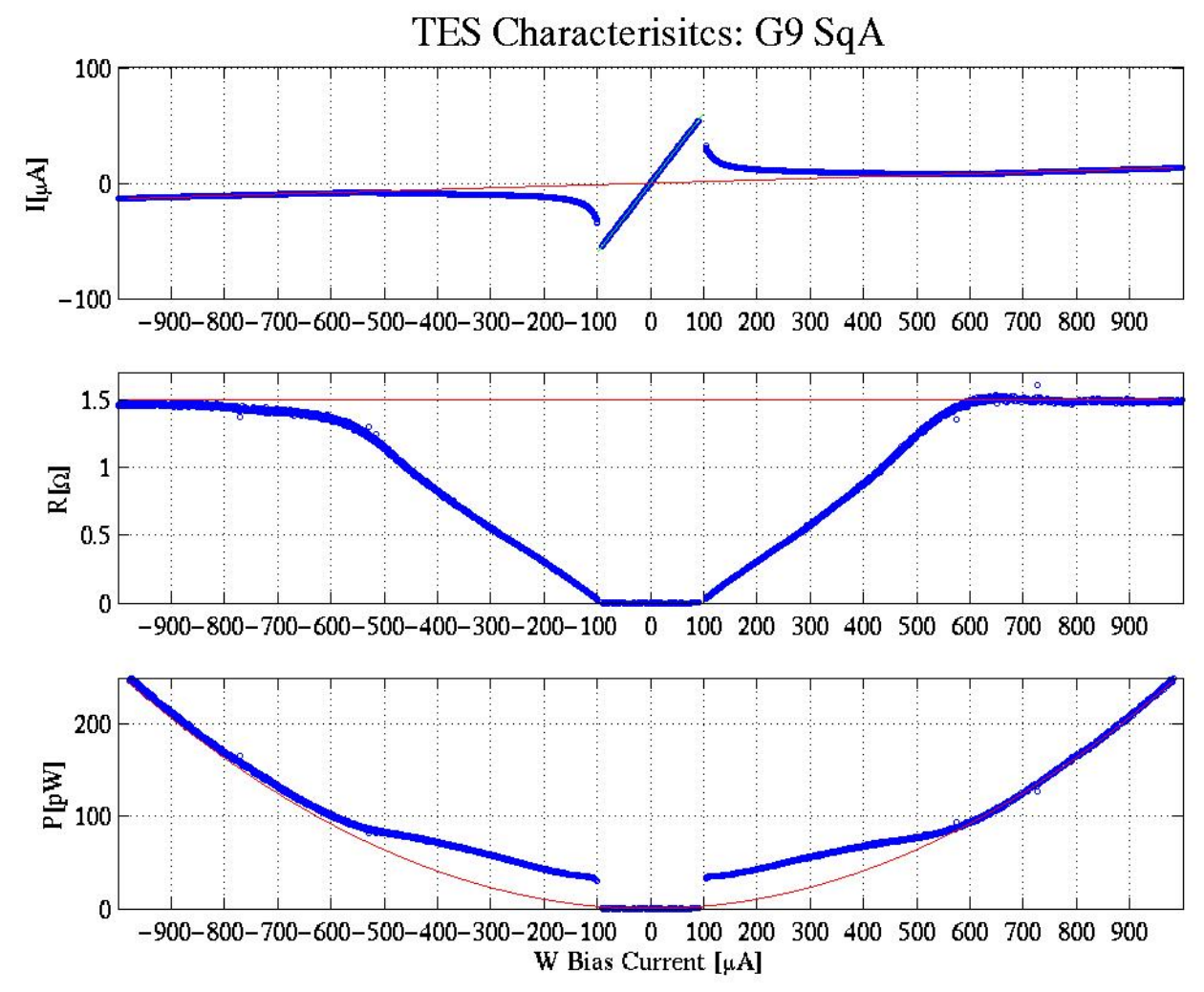

Figure 5.4: IbIs Curves for Detector Z5 (G9), Channel A (post-implant). The dark blue circles represent the data. The $\mathrm{x}$-axis in all three curves is the bias current (see Figure 5.1) and the top graph is of the current measured in the sensor. The red line is a linear fit to the part of the curve when the large bias current has driven the sensor normal, as seen in the calculated graph of resistance-vs.-bias current (middle). Near zero bias current, the slope of the sensor current represents a small parasitic resistance $(\sim 10 \mathrm{~m} \Omega)$. The bottom graph is of the calculated power dissipation in the sensor, which is parabolic in the normal region $\left(P=I^{2} R\right)$. For sufficiently narrow transitions, the power is approximately constant in the transition region (see text). The fact that the power is curved within the transition region indicates the existence of a $T_{c}$ gradient in that quadrant. 


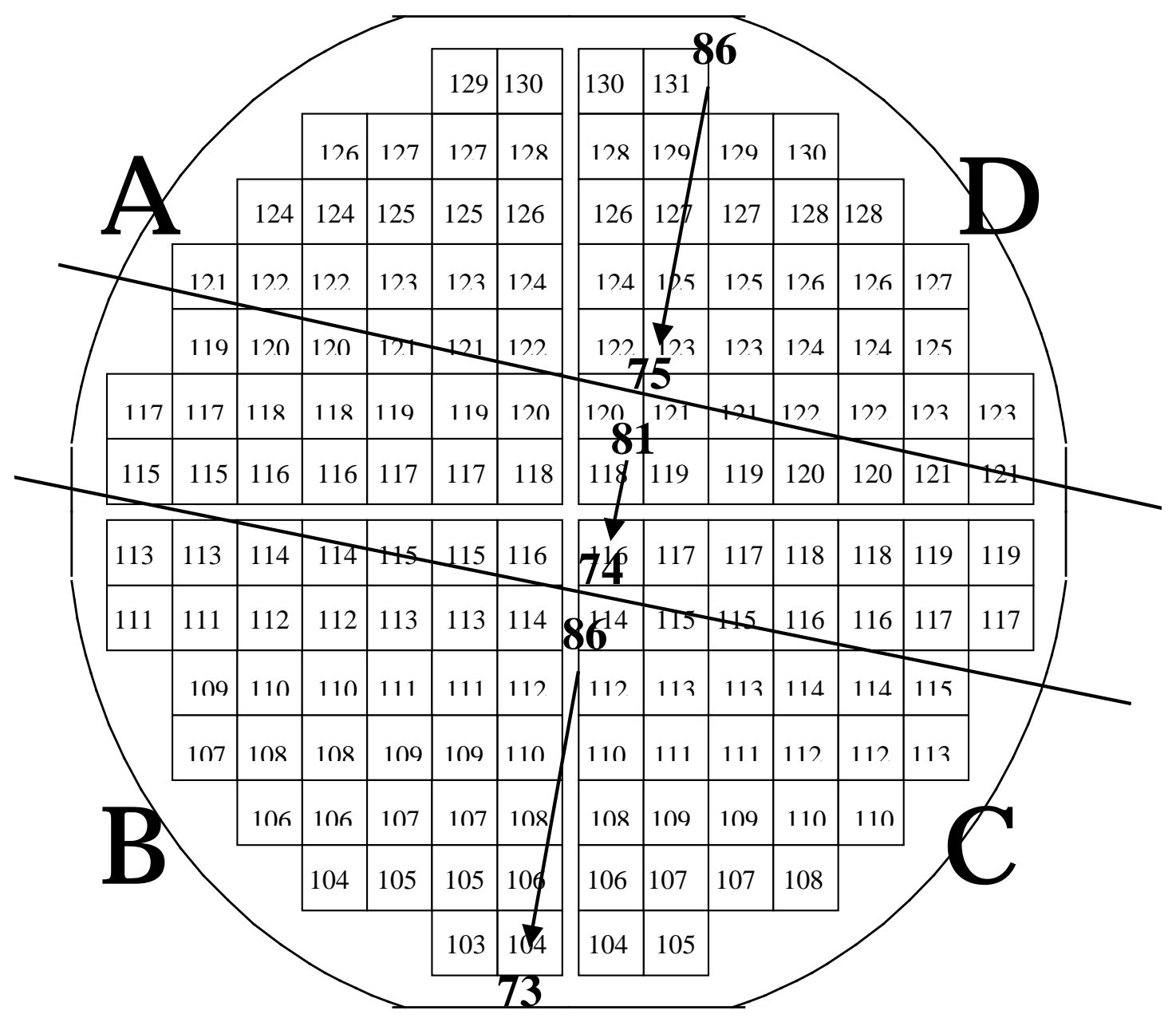

Scribed major flat

Figure 5.5: Simulated $T_{c}$ Distribution for Z5 (G9) and Planned Implantation Scheme. The modeled $T_{c}$ distribution is shown with a proposed three-mask implantation scheme. The data in Table 5.2 show that the implantation successfully reduced both the overall $T_{c}$ range as well as the severity of the $T_{c}$ gradient [7]. 


\section{Works Cited}

[1] K.D. Irwin. Phonon-Mediated Particle Detection Using Superconducting Tungsten Transition-Edge Sensors. PhD thesis, Stanford University, February 1995.

[2] T. Saab. Search for Weakly Interacting Massive Particles with the Cryogenic Dark Matter Search Experiment. PhD thesis, Stanford University, August 2002.

[3] B.A. Young et al. Effect of implanted metal impurities on superconducting tungsten films. Journal of Applied Physics, 91(10), May 2002.

[4] P.L. Brink et al. Determination of the tc distribution for 1000 transition edge sensors. In Ninth International Workshop on Low Temperature Detectors, volume 605, pages 529-532. American Institute of Physics, July 2001.

[5] R.P. Welty and J.M. Martinis. Two-stage integrated squid amplifier with series array output. IEEE Transactions on Applied Superconductivity, 3(1):2605-2608, March 1993.

[6] T. Saab. Ibis curves as a useful sensor diagnostic. CDMSnote \#9911001, November 1999 .

[7] Paul Brink et al. G9 w tc distribution, before ion-implantation. Internal note, May 2001. 


\section{Chapter 6}

\section{Background Discrimination}

The initial cooldown of the six detectors chosen for Run 21 began in July of 2001. Before beginning data-taking for a dark-matter search, we performed a series of calibrations in order to establish the separation of the electron recoil and nuclear recoil bands in the yield parameter. Our detectors are exposed to a ${ }^{60} \mathrm{Co}$ source to produce a population of gamma events and $\mathrm{a}^{252} \mathrm{Cf}$ source to produce a population of neutron events. We use these events from a known source to establish bands in the yield-vs.-energy plane which contain $99 \%(3 \sigma)$ of the electron recoils and $95 \%(2 \sigma)$ of the nuclear recoils. The separation of these bands allows us to reject better than 99.99\% of the gamma-like events in our nuclear recoil band, which is also our WIMP signal region.

Roughly half of the low-background data taken in Run 21, previously analyzed and reported in the Ph.D. Thesis of Tarek Saab [1] as well as in [2], was taken with 3V charge bias 1 . The other half, which is being reported for the first time in this thesis, was taken at $6 \mathrm{~V}$ bias. The main difference which results from running at different voltage biases is the effect of the Neganov-Trofimov-Luke (NTL) phonons [3] 4]. Theoretically, we would expect no difference in sensitivity between the two voltages, but there could be differences in the noise, saturation of the phonon sensors, etc. Part of the purpose of running at two voltages was to accumulate experimental data as to which voltage would be best for running at our deep site.

Since the previous reporting, there have been changes to the analysis method, particularly in the analysis of beta contamination detailed in the following chapter. In order to preserve blindness in making cut levels, etc., a new analysis method was developed on the $6 \mathrm{~V}$ data first and then later applied in an automated fashion to the $3 \mathrm{~V}$ data.

\footnotetext{
${ }^{1}$ This bias refers to the germanium detectors. The silicon detectors were run at $4 \mathrm{~V}$ for the whole of the Run.
} 


\subsection{Event Reconstruction}

The CDMS data acquisition outputs six data channels of raw data for each detector - digitizer traces for each of the two charge channels and the four phonon channels. The pulses themselves are $1.638 \mathrm{~ms}$ long digitized at a rate of $1.25 \mathrm{MHz}$ (2048 data points). Figure 6.1 shows a sample event with traces in all six detectors where there has been a summation over the two charge channels and the four phonon channels. In addition, a large quantity of associated information, such as biasing levels and veto and trigger history buffers are stored. Once the raw traces have been written to disk, a Perl routine automatically starts large collection of analysis programs in Matlab (C) which take the output of the DAQ and convert it into Reduced Quantities (RQs) which are relevant for physics analysis. For this analysis, we are concerned with three main quantities, the energy in the charge channels, the energy in the phonon channels, and the phonon pulse risetimes. The phonon pulse arrival times (delay) are also used in correcting for any position variation across the surface of the detector, based on a speed-of-sound reconstruction.

An optimal filter algorithm was used to fit a template pulse to our data. The template pulse in the phonon channels has been constructed to the approximate form seen in the data:

$$
T_{p}(t)=\frac{1}{A} \Theta(t)\left(-e^{\left(t-t_{0}\right) / \tau_{r t}}+e^{\left(t-t_{0}\right) / \tau_{f t}}\right)
$$

where $\tau_{r t}$ is the risetime of the pulse $(\sim 32 \mu s$ for Ge and $\sim 25 \mu s$ for $\mathrm{Si}$, depending slightly on the observed pulse shapes in a particular detector) and $\tau_{f t}$ is the falltime of the pulse ( $\sim 170 \mu s$ for Ge and $\sim 130 \mu s$ for $\mathrm{Si}$ ). The exact shape of the pulse depends on the physics of the interaction, so the phonon templates were normalized to have $\int T d t=1$ since our phonon pulses are expected to have some variation in shape but the integral of the pulse is expected to correspond to the energy (see Equation 4.8). The charge templates are normalized to $\max (T)=1$ and are created by summing a large sample of pulses which are chosen to have the appropriate properties (inner electrode or outer electrode only). There is a known cross-talk between events in the two electrodes of the same detector, so template pulses for the two crosstalk channels are made as well and the optimal filter routine takes this crosstalk into account when calculating the energy [5].

The templates are then scaled such that the output of the optimal filter routine converts the raw pulse from "digitizer units" to "physics units" (keV). The basic energy calibration of the detectors was done using the $662 \mathrm{keV}$ gamma line from a

${ }^{137} \mathrm{Cs}$ source [1]. While this energy is outside the energy range of interest for WIMP studies $(E<100 \mathrm{keV})$, it is strong enough to be used as an external source since it can penetrate the copper cans of the refrigerator. This line is visible in germanium detectors, but such a high-energy gamma has a very low interaction probability in 


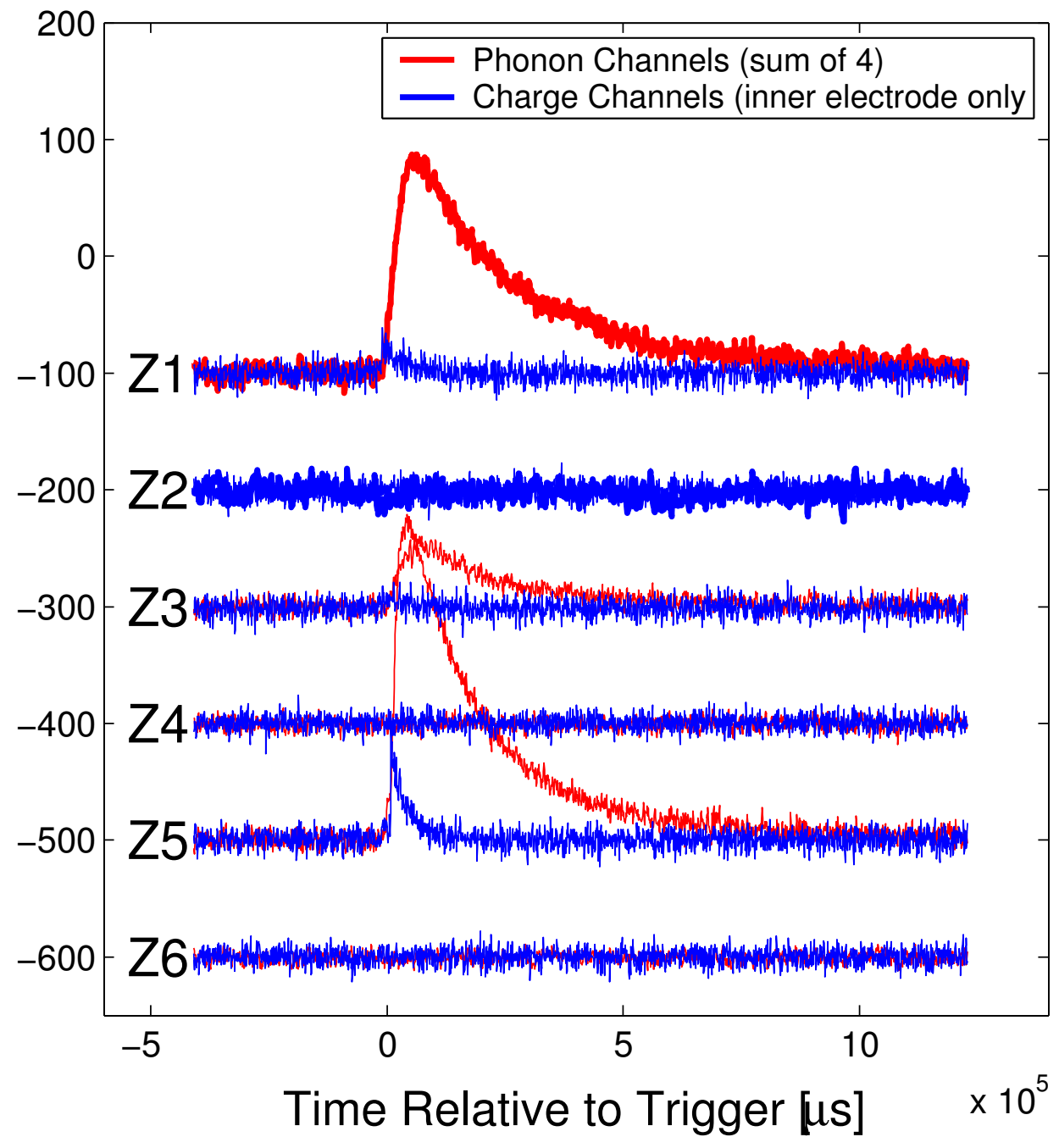

Figure 6.1: Sample Pulses for the Charge and Phonon Sensors for all Six Detectors. The red curves are the sum of the four phonon channels and the blue curves are the q-inner channel. Each pulse consists of 2048 data points digitized at a rate of $1.25 \mathrm{MHz}$. 
the silicon detectors. We used a Monte Carlo simulation to make a spectrum so that we could compare other features like the Compton edge to set the energy scale. The phonon channels are tuned to be sensitive at lower energies, so they experience nonlinearities at $662 \mathrm{keV}$. The calibration constants were tuned slightly during the run when other features, such as lines in the calibration data, became available. Our final energy calibration had an uncertainty of only a few percent [1].

Optimal filtering fits the pulse in the frequency domain where each component is weighted by the noise at that frequency [6]. A correlation in frequency space $(\hat{C})$ between the pulse $(P)$ and a noise-weighted template $\left(T^{\prime}\right)$ yields:

$$
\hat{C}\left(\omega_{j}\right)=\hat{P}\left(\omega_{j}\right) \hat{T}^{\prime}\left(\omega_{j}\right)=\frac{\hat{P} \hat{T}}{\hat{N}^{2}} .
$$

where $\hat{T}=\mathcal{F}(T)$ is the Fourier transform and $\hat{N}$ is the noise spectrum. In order to keep the units correct we must define a normalization factor $a$ :

$$
a=\frac{\hat{A}=\hat{T}^{2} / \hat{N}}{\max (\operatorname{Re}(A))}
$$

where $\operatorname{Re}(A)$ is the real part of $A$ such that when we convert back to the time-domain via the inverse Fourier transform $T=\widehat{\mathcal{F}}(\hat{T})$ we have:

$$
C^{\prime}\left(t_{i}\right)=a \operatorname{Re}(C)=\frac{\operatorname{Re}\left(\widehat{\mathcal{F}}\left(\frac{\hat{P} \hat{T}}{\hat{N}^{2}}\right)\right)}{\max \left(\operatorname{Re}\left(\hat{\mathcal{F}}\left(\frac{\hat{T}^{2}}{\hat{N}}\right)\right)\right)}
$$

Because we have been careful about the units, we can simply look for the peak of this correlation function and read off the energy $\left(E_{p h}[\mathrm{eV}]=\max \left(C^{\prime}\left(t_{i}\right)\right)\right)$ and the delay parameter $\left(t_{i}\right)$. The peak for the charge channels are found for the sum of the inner and outer electrodes and then the energies and crosstalk correction are found for both channels at that single delay time.

While we measure the phonon energy, this is not the same as the recoil energy deposited by the collision. In Chapter 4, we discussed the NTL phonons which are created by the passage of the electron-hole pairs as they drift towards the electrodes. As described in Equation 4.1, we can find the recoil energy deposited by the collision from:

$$
E_{\text {recoil }}=E_{\text {phonon }}-\frac{E_{q}|V|}{E_{e-h}}
$$

The risetime is found via a simple walk-search program which then interpolates 
the location of the $10 \%$ and $40 \%$ levels. Similarly, the phonon pulse arrival times are defined as the time relative to the trigger at which the pulse goes above the $20 \%$ level. Our phonon sensors are segmented into quadrants, so we can use the relative timings of the four pulses to extract position information about the event location. There exists a degeneracy in the position as inferred from the delay parameter, but we can use the relative energies of the pulses in each quadrant to break that degeneracy.

The testing process has already shown that the phonon energy response is not uniform over the detector due to variations in the individual TESs which comprise the phonon sensors. With the assumption that the charge energy does not have a position dependence, we constructed a correction matrix for the recoil energy and the risetime based on position [7]. Using the data from the gamma calibration source, we compared the phonon energy expected from the charge energy $\left(E_{p}=\frac{y}{E_{q}}\right.$ with $y \approx 1$ for electron recoils) to the measured phonon energy. We then created a lookup table with an averaging over the 16 nearest neighbors. A similar correction matrix was applied to the risetime.

\subsection{Search Thresholds}

We define search thresholds at the minimum level which the optimal filter routine reliably finds a true pulse above the noise. Randomly-triggered data provide us with a sample of noise traces with which to produce a distribution of noise levels. This distribution is not be centered on zero because the optimal filter routine finds the highest "peak" in a trace even if there is no pulse present. We want to choose our threshold level such that we have a vanishingly small chance of misidentifying a noise event as a true pulse. We fit a Gaussian to the data near zero energy from the random triggers. We then scale the livetime to the number of events in the region $\pm 2 \sigma$ in the low-background data and calculate the expected number of noise events above threshold per 100 livedays (approximately the livetime of our experiment).

Figures A.1 and A.2 show the phonon noise levels and Figure A.3 iand A.4 show the charge noise levels for each of the six detectors. Tables A.1 and A.2 show the expected number of leakage events per 100 livedays of data in each quantity and at each voltage (approximately the livetime of the experiment). The $6 \sigma$ thresholds, chosen to conservatively limit our leakage, are shown in Table 6.1. The levels between the two bias voltages were sufficiently similar that the higher of the two thresholds was chosen. 


\begin{tabular}{|c|c|c|}
\hline & $\begin{array}{c}\text { Phonon } \\
\text { Threshold }\end{array}$ & $\begin{array}{c}\text { Charge } \\
\text { Threshold }\end{array}$ \\
\hline Z1 $(\mathrm{Ge})$ & $2.5790 \mathrm{keV}$ & $1.5008 \mathrm{keV}$ \\
\hline Z2 $(\mathrm{Ge})$ & $0.7983 \mathrm{keV}$ & $1.3624 \mathrm{keV}$ \\
\hline Z3 $(\mathrm{Ge})$ & $0.9589 \mathrm{keV}$ & $1.6634 \mathrm{keV}$ \\
\hline Z4 $(\mathrm{Si})$ & $1.8182 \mathrm{keV}$ & $1.9285 \mathrm{keV}$ \\
\hline Z5 $(\mathrm{Ge})$ & $0.8445 \mathrm{keV}$ & $1.5431 \mathrm{keV}$ \\
\hline Z6 $(\mathrm{Si})$ & $1.6243 \mathrm{keV}$ & $2.7094 \mathrm{keV}$ \\
\hline
\end{tabular}

Table 6.1: Phonon and Ionization Search Thresholds. $6-\sigma$ thresholds were chosen to limit the leakage of noise triggers into the data to less than one event per 100 livedays. The levels between the two bias voltages were sufficiently similar that the higher of the two thresholds was chosen.

\subsection{Fiducial Volume Cut}

In Chapter 3 we noted that we could use portions of the detectors as shielding of lowenergy backgrounds. We exclude events which occur in the outer charge electrode, as depicted in Figure 6.2. We use the energy in the inner charge electrode $\left(q_{i}\right)$ as the measure of the ionization energy rather than $q_{\text {sum }}=q_{i}+q_{o}$ since we are excluding events with a signal in the outer electrode above the noise threshold. This gives us an effective $\sqrt{2}$ improvement in our noise.

Events which deposit energy in the outer electrode are excluded by establishing noise levels in each electrode and keeping events which are above the noise in $q_{i}$ and consistent with noise in $q_{o}$. Above our search threshold in $q_{i}$, there is a band of events with no energy deposited in $q_{o}$, but with some noise value for the energy. This band is centered on zero since the optimal filtering routine has already found a pulse in $q_{i}$ and the energy in $q_{o}$ is found at that same time. The band is not flat because there is some energy dependence in the crosstalk which is not corrected for and depends strongly on detector and weakly on bias voltage. The effect is particularly noticeable in detector $\mathrm{Z} 2$, which is the reason a more complicated shape for the bands was chosen.

The bands are fit by first taking $10 \mathrm{keV}$ energy slices in $q_{i}$ and fitting a Gaussian to the peak near zero. The centers of these Gaussians are fit by a power law $\left(A q_{i}^{B}\right)$ and the widths by a straight line $\left(C q_{i}+D\right)$ to make our "q-outer noise band." Figures A.5 and A.2 show the results of the fitting routine.

One thing to note is the significant number of events with energies below the band. Events above the band are easy to understand since there are events in a "shared" region for which energy is deposited in both electrodes. These events below the band occur at a particular region on the detectors where the electrical ground (provided by the phonon sensors, see Chapter 4) was floating. The detectors were 


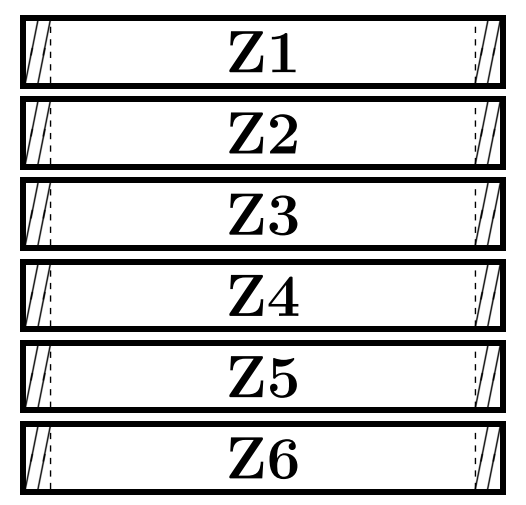

Figure 6.2: Side View of the Fiducial Volume Cut. The detectors and spacing are drawn to scale, with the inner electrode containing approximately $85 \%$ of the volume of the detector. The hatched regions show the excluded outer charge electrode of each detector.

designed such that the surface conductance of the detectors would provide a sufficient connection, but the evidence suggests otherwise, as shown in Figure 6.4. Events in these areas have energy in the inner electrode but do not have the associated q-outer crosstalk [8] 9]. The optimal filter routine then produces a negative value for $q_{o}$ since it "corrects" the noise in $q_{o}$ found at the $q_{i}$ delay time. Since the crosstalk correction is effectively

$$
\begin{aligned}
& q_{i}^{\prime}=q_{i}+X_{o i} q_{o} \\
& q_{o}^{\prime}=q_{o}+X_{i o} q_{i}
\end{aligned}
$$

we keep these "low- $q_{o}$ " events since the value of $q_{i}$ is unchanged.

Finally, we would like to know the efficiency of this cut, that is, what is the percentage of events that we are cutting that we think are really "q-inner-only" events. The events which are above the band are most likely events which have a true signal in the outer electrode. The plot of efficiency-vs.-energy in Figure 6.5 is constructed by comparing our cut against events which are less than the $3 \sigma$ upper limit of the noise band. This should be a good estimate of the number of "true q-inner" events and allow us to take into account any variations of the shape of the bands versus our fit functions as well as including all of the events below the band. Given that we have chosen a $2 \sigma$ width for our noise band, we would expect an efficiency near $97.5 \%$ taking into account that we have included the "low- $q_{o}$ " events. 


\section{CDMS Run 21 - 3V Low-Bkgnd. Data - Z4}

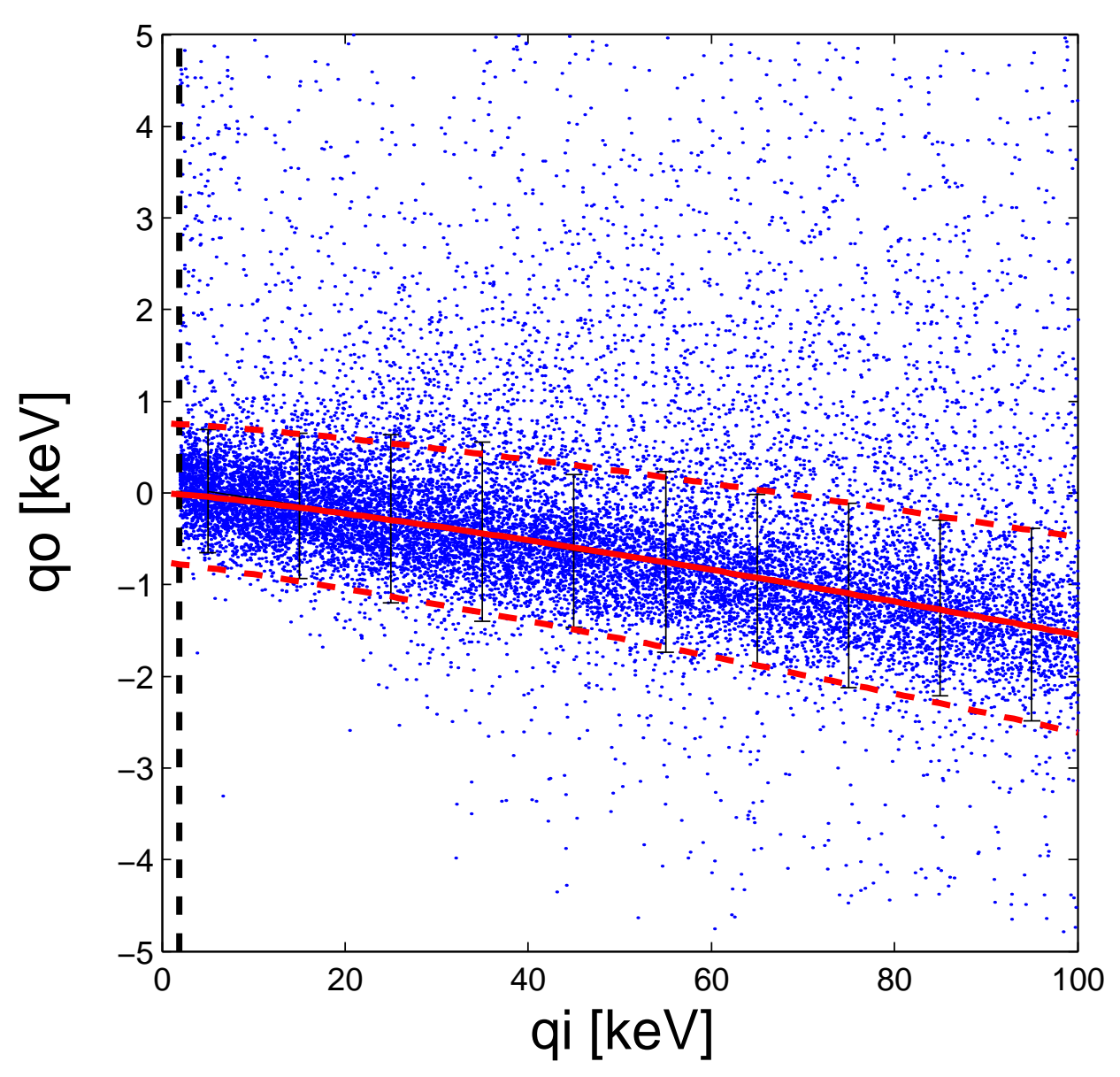

Figure 6.3: A Sample Fit to the Fiducial Volume Cut. The visible band in the data points (blue) represent events contained within the inner charge electrode. They have qi (energy deposited in the inner electrode) greater than a $6 \sigma$ noise threshold, but have qo (energy deposited in the outer electrode) consistent with a $2 \sigma$ noise band. The qi threshold is shown as a vertical dash line and the $\pm 2 \sigma$ qo noise band are shown as the dashed curves. Note that there is a significant population of events below the qo noise band. These events are actually contained within the inner electrode, but an error in the detector construction leads them to have a low value of qo (see Figure 6.4). Based on this data, the next batch of detectors has had this problem corrected. 

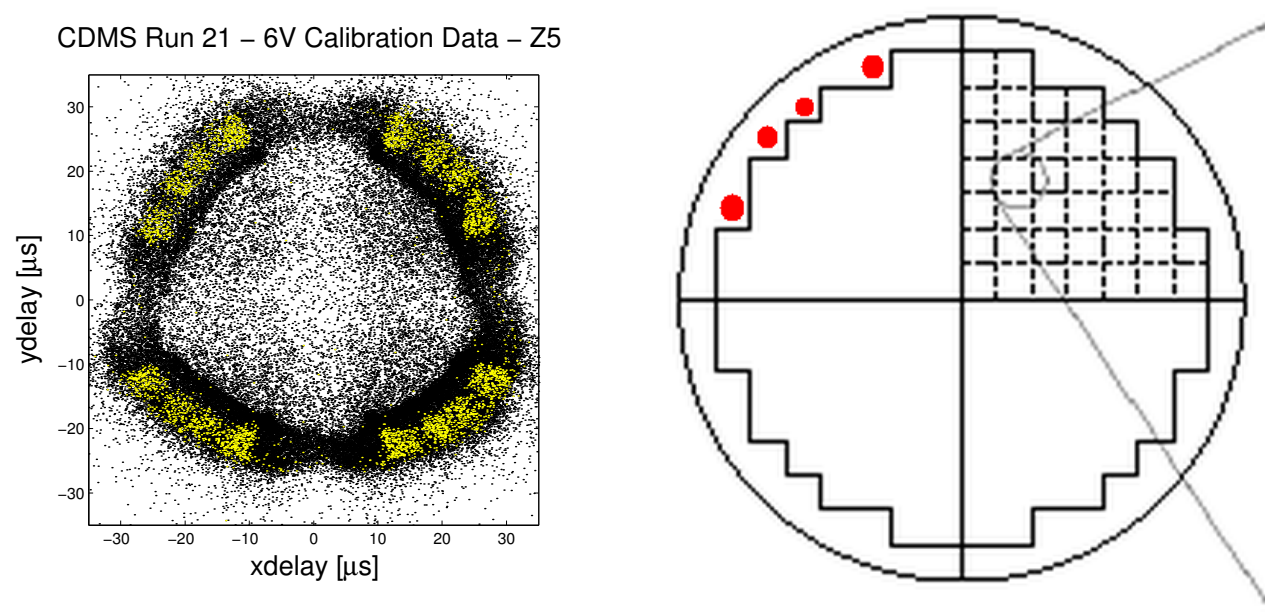

Figure 6.4: Comparison of Over-corrected Crosstalk with Position. The left plot shows the location of events which are below the q-outer noise band and the right plot shows the location of the floating ground areas of the detector. Subsequent detectors will have the metalized surfaces at these locations connected to ground, thus correcting the problem.
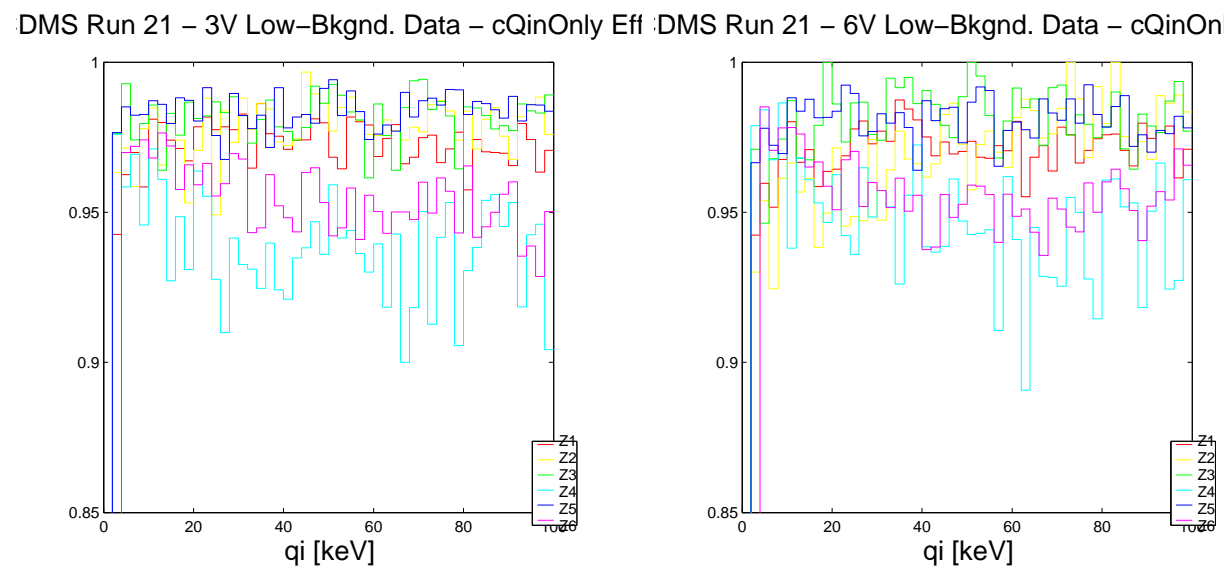

Figure 6.5: Efficiency of the Fiducial Volume Cut versus Energy. An estimate of the number of "true q-inner" events is made by taking all events below the $3 \sigma$ upper limit of the noise band. This number is compared to the $2 \sigma$ noise band shown in Figures A.5 and A.2 to make an efficiency which is predicted to be near $97.5 \%$. 


\subsection{Gamma Calibration}

As discussed in Chapter 4, our main background discrimination parameter is the ratio of the charge signal to the phonon signal. We call this quantity the "yield," $y=q / p$. The CDMS experiment has shown that this quantity can be used to clearly discriminate background events (electron recoils) from candidate signal events (nuclear recoils) (e.g. [2]). Detector response varies slightly from detector to detector, so we use calibration sources which are similar to the events we wish to characterize to define our discrimination levels.

We use gammas from a ${ }^{60} \mathrm{Co}$ source, which has two strong lines at $1.1 \mathrm{MeV}$ and $1.3 \mathrm{MeV}$, to define our electron recoils. As with the ${ }^{137} \mathrm{Cs}$ source used in the energy calibration, this is not in our range of interest $(E<100 \mathrm{keV})$, but it is strong enough to penetrate the copper cans of the cryostat. Multiple Compton scattering with the intervening material produce a spectrum of events below $100 \mathrm{keV}$ (Figure 6.6).

In addition to some basic data quality cuts (cGoodEv(det)), we make three cuts on the data to select a clean sample. First, we make our fiducial volume cut (cQinOnly(det)), not only to use the outer guard ring as a shield, but also to get rid of events which may have poor charge collection near the edge of the detector. We make a veto cut (cAntiCo) to exclude cosmic ray-induced electromagnetic showers or other high-energy particles (Chapter 3) which might pollute our sample of gammas. Finally, a singles cut (cPSingle), accepting events for which only one detector has an energy above threshold, is made.

With these cuts, a plot of yield versus recoil energy was sliced into energy bins and a Gaussian was fit to the yield histogram in each energy bin. The data from the energy bins are then fit using the same function used in the previous analysis [1]:

$$
\begin{array}{r}
y_{c}=a E_{r}^{b-1} \\
\mu_{y}=\sqrt{c^{2}+d^{2} E_{r}^{2}} / E_{r}
\end{array}
$$

where $\mathrm{a}, \mathrm{b}, \mathrm{c}$, and $\mathrm{d}$ are constants for each individual detector. $y_{c}$ is the centroid of the band and $\mu_{y}$ is the (symmetric) width of the band. The bands are almost flat, so we expect $b \sim 1$. It is interesting to note that the uncertainty in the yield diverges at low energies rather than high because the uncertainties in charge and phonon energies are uncorrelated and hence:

$$
\delta y=\delta\left(\frac{q}{p}\right)=\sqrt{\left(\frac{1}{p} \delta q\right)^{2}+\left(\frac{q}{p^{2}} \delta p\right)^{2}}=\sqrt{\left(y \frac{\delta q}{q}\right)^{2}+\left(y \frac{\delta p}{p}\right)^{2}}
$$

where we now have our functional form vs. $p$. Mapping these back to our constants,

we have $\delta q=c$ and $\delta p=\frac{d p}{y}=\frac{d p^{2}}{q}$. Figure 6.7 shows these components (for a 


\section{CDMS Run $21-{ }^{60}$ Co Calibration data - Z5}

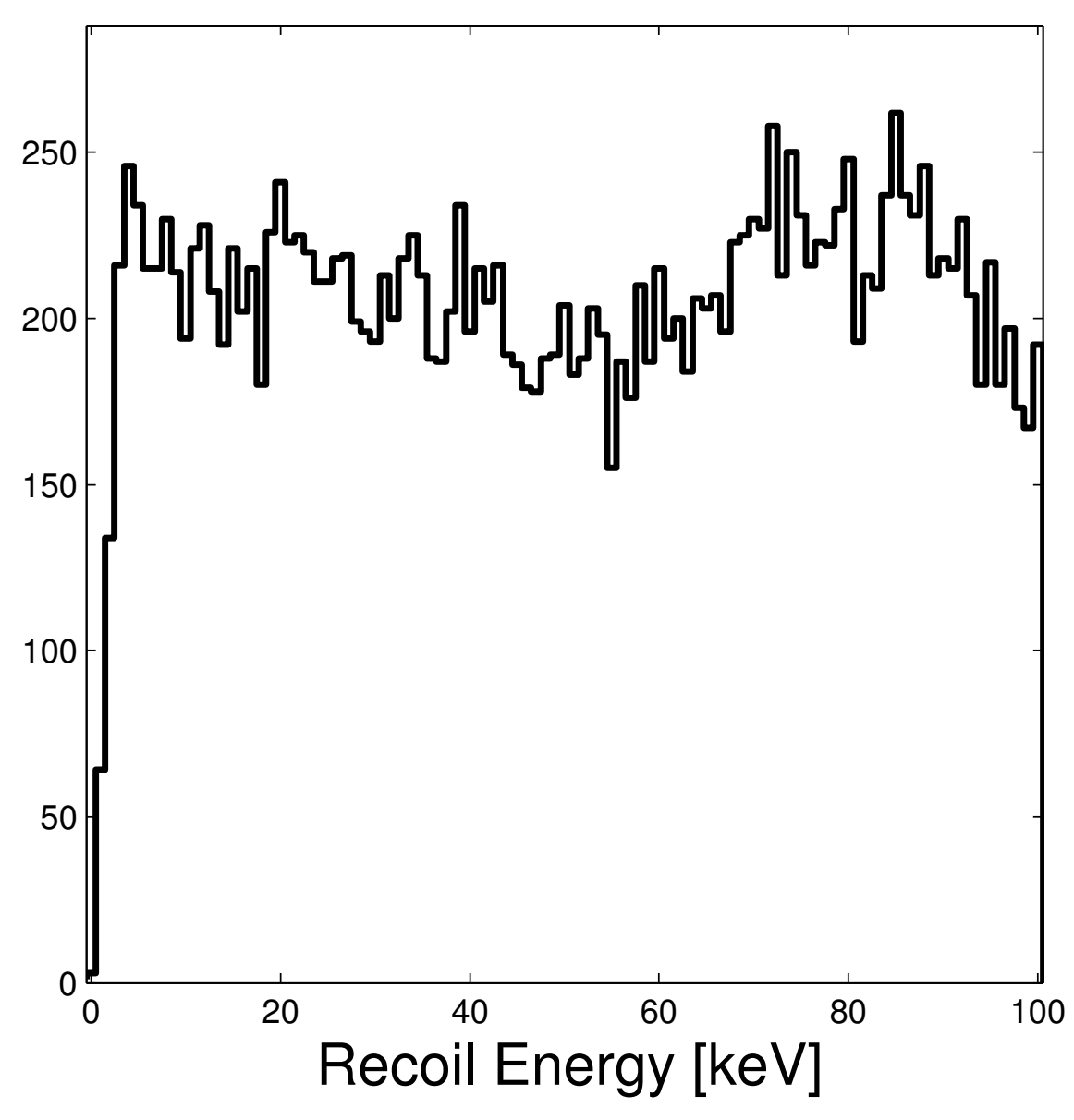

Figure 6.6: A Sample ${ }^{60}$ Co Spectrum. The spectrum is shown for the energies of interest for WIMP detection, less than $100 \mathrm{keV}$. The ${ }^{60} \mathrm{Co}$ energy spectrum is well-defined external to the experimental volume, but scattering processes in the intervening material produce a continuum of energies in the range of interest. 
nuclear recoil band, which has the same form) on a yield-vs.-recoil energy plot with the expected behavior with respect to energy. The bands are not perfect since the fits to Equation 6.9 were done in slices of recoil energy, essentially treating the noise as being all in the charge. This should not be a problem since the excess is on the high side of the band and we are primarily concerned with preventing leakage below the band.

The final electron recoil bands are shown in Figure 6.8 (3V) and Figure 6.9, with the fits superimposed on the calibration data. Detailed plots of the fitting procedure including histograms of the energy bins can be found in Appendix A.

\subsection{Neutron Calibration}

The procedure for the calibration with the ${ }^{252} \mathrm{Cf}$ source was almost the same as the ${ }^{60} \mathrm{Co}$ calibration with two main differences. First, we must limit the exposure of the detectors to a neutron source since the germanium detectors can capture neutrons and transmute the stable ${ }^{72} \mathrm{Ge}$ to ${ }^{73} \mathrm{Ge}$, which emits a $10 \mathrm{keV}$ gamma. This feature is visible in our low-background data. With fewer events than in our gamma calibration, we widened the energy bins, especially at higher energies. Second, the neutron data contains a population of background gammas, so we restricted the events to be below the previously fit electron recoil band. We use the same shape for the bands as used for the electron bands above (see Equation 6.9).

The final nuclear recoil bands are shown in Figures 6.8 and 6.9, with the fits superimposed on the calibration data. Detailed plots of the fitting procedure including histograms of the energy bins can be found in Appendix A.

\subsection{Population of the Bands in the Calibration Data}

The 2-D histograms of the combined neutron and gamma calibrations, shown in Figure 6.10, represent an excellent separation between the bands. 2-D histograms for the gamma calibration alone are also shown. With the fits for the electron recoil and nuclear recoil bands overlaid, we can see that there is very little leakage of gamma events down into the nuclear recoil band. Appendix $\mathrm{A}$ has the detailed plots for each separate detector and tables with the population of different regions of the yield-vs.-energy plane. Most importantly, the two (five) leaked events in germanium (silicon) out of a total $72,410(17,813)$ events represents a gamma rejection efficiency of $99.997 \%(99.97 \%)$. 


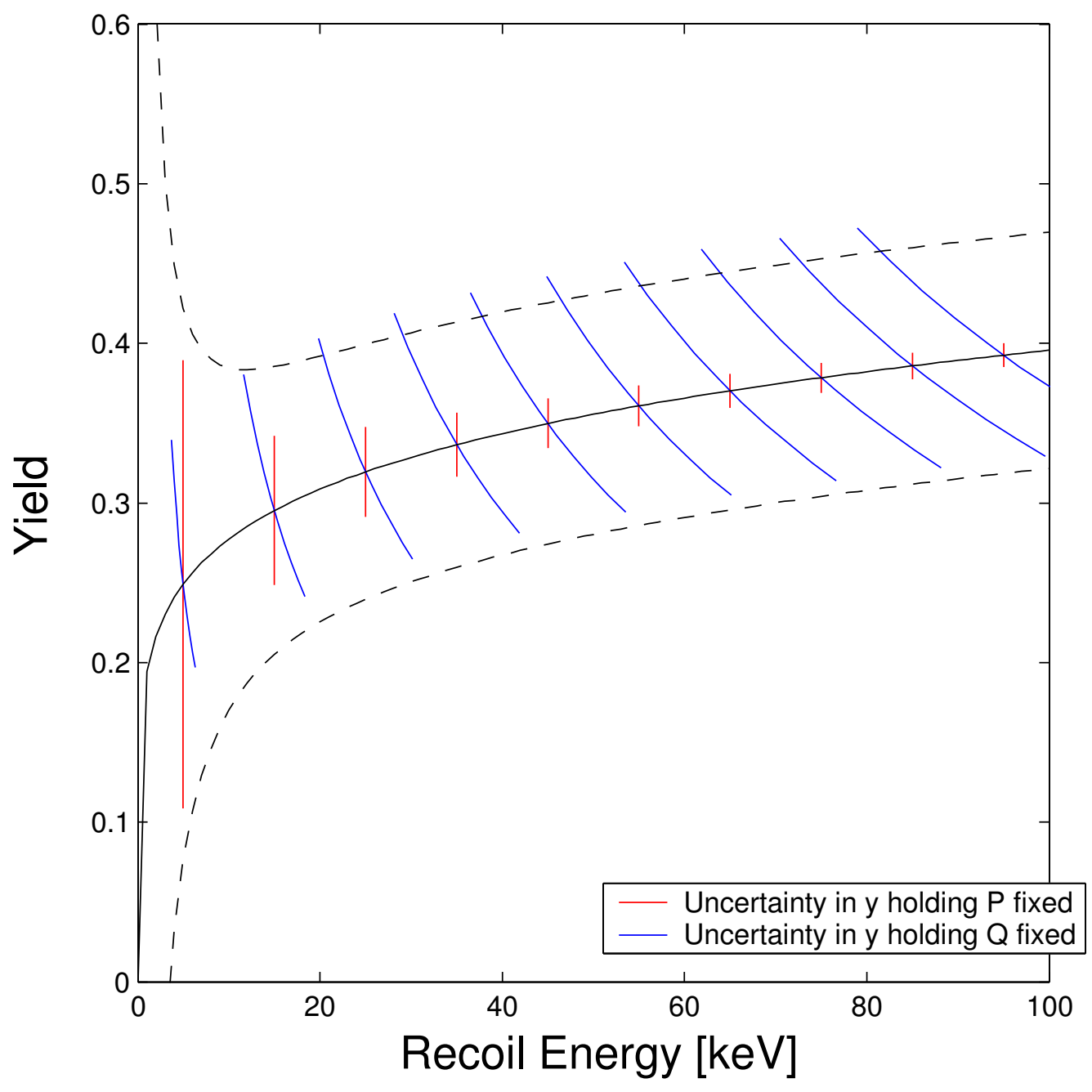

Figure 6.7: Uncertainties in the Band Widths. The noise is contained within the charge measurement $(Q)$ and the phonon measurement $(P)$, while our standard graph is of yield $(Y=Q / P)$ vs. recoil energy $(\propto P)$. Shown are the individual components of the uncertainty in $Q$ (red vertical lines) and $P$ (blue hyperbolic lines $-\frac{d Y}{d Q} \propto \frac{1}{P}$ ) for fixed values of the recoil energy. The nuclear recoil band shown does not exactly match these curves because the bands were based on a fit which puts all of the noise in the charge channels. This was done for the sake of simplicity, and appears to be a good approximation. 

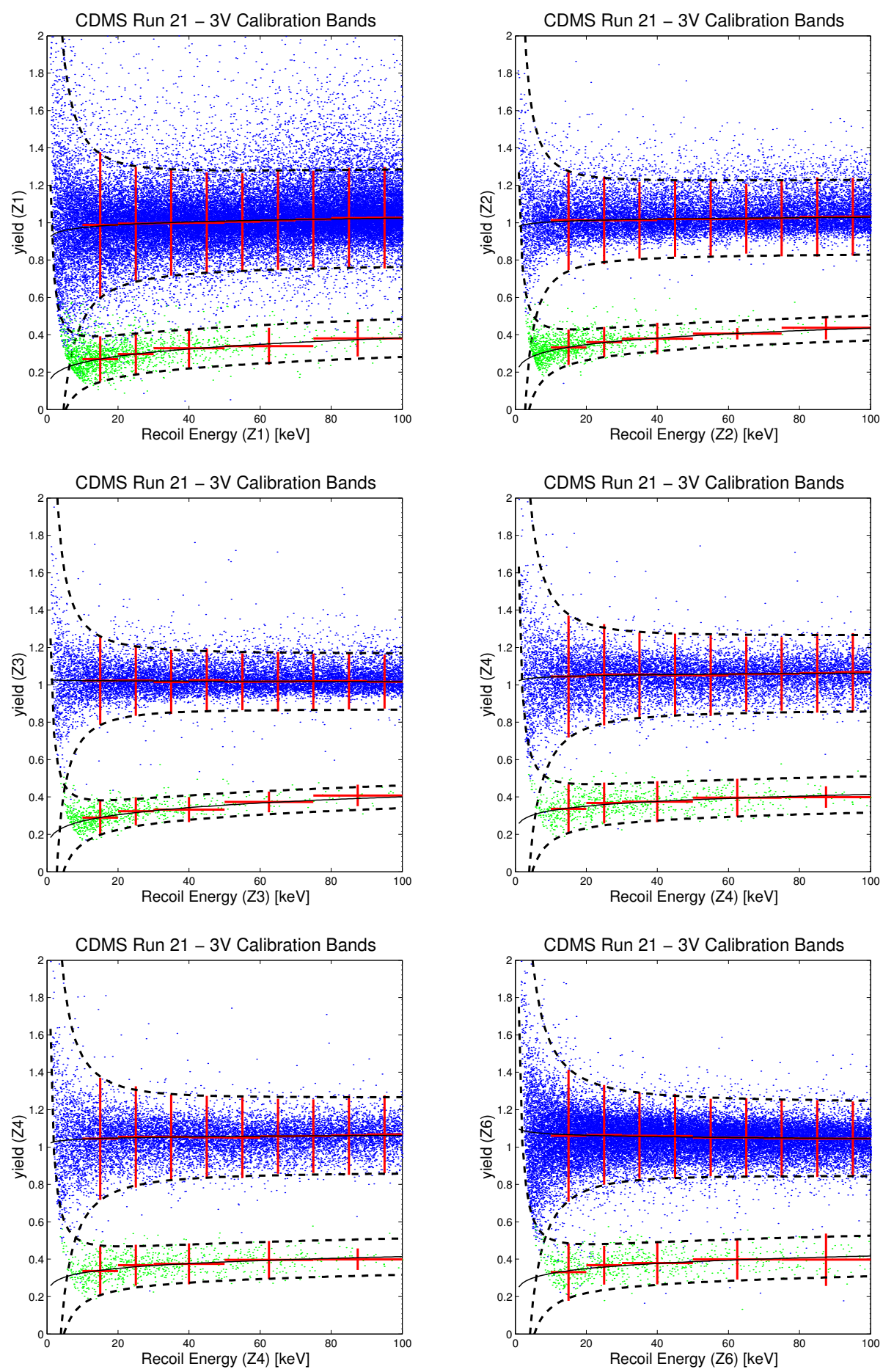

Figure 6.8: Electron Recoil and Nuclear Recoil Bands in the 3V Calibration Data. The blue dots are events from the gamma calibration and the green dots are events from the neutron calibration (with a cleaning cut to exclude most of the events in the electron recoil band). The red crosses represent the energy range and width ( $3 \sigma$ for the electron recoil band and $2 \sigma$ for the nuclear recoil band) of a Gaussian fit to the band, and the black dashed curves represent the resulting fit. 

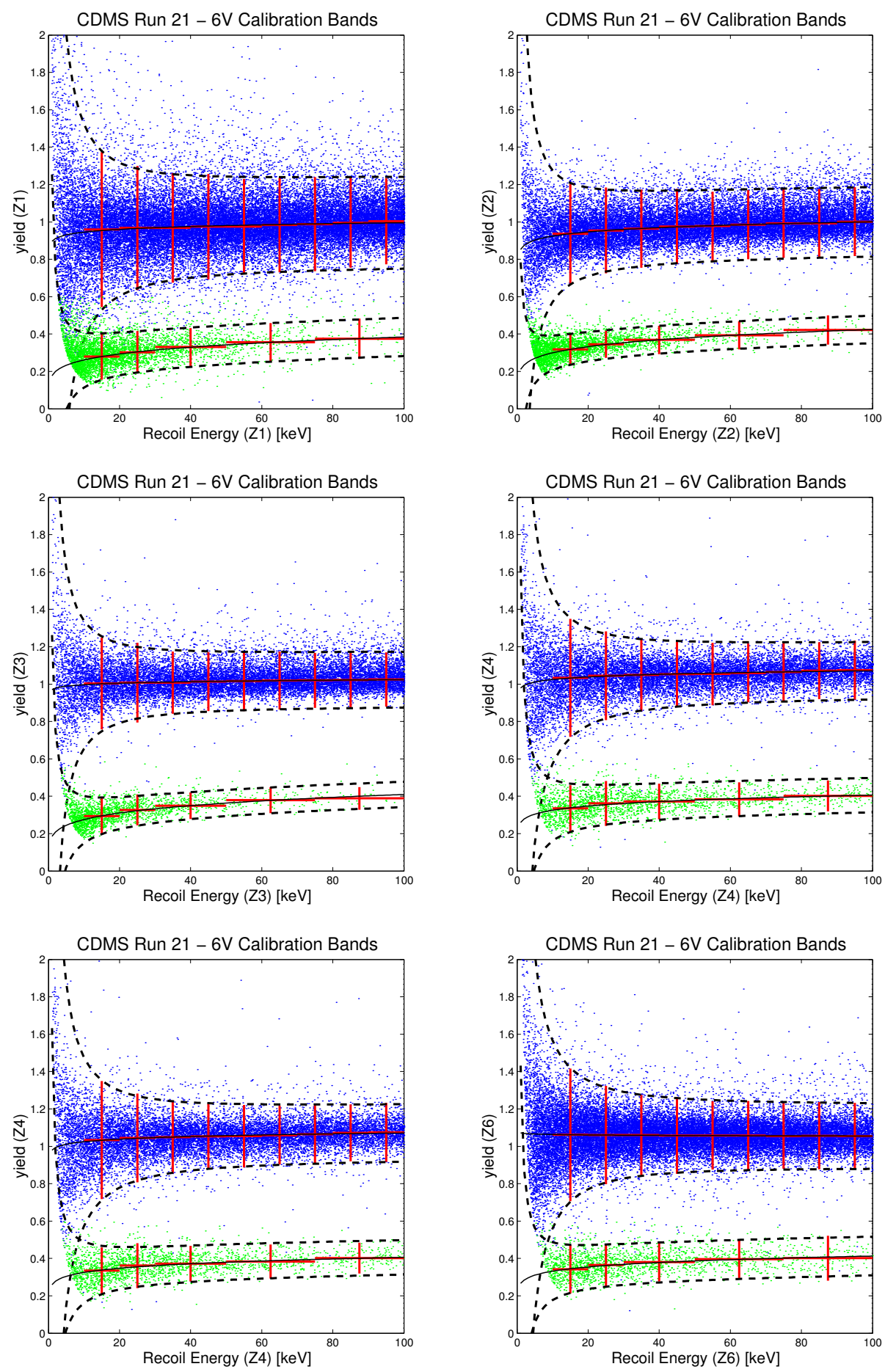

Figure 6.9: Electron Recoil and Nuclear Recoil Bands in the 6V Calibration Data. The blue dots are events from the gamma calibration and the green dots are events from the neutron calibration (with a cleaning cut to exclude most of the events in the electron recoil band). The red crosses represent the energy range and width ( $3 \sigma$ for the electron recoil band and $2 \sigma$ for the nuclear recoil band) of a Gaussian fit to the band, and the black dashed curves represent the resulting fit. 

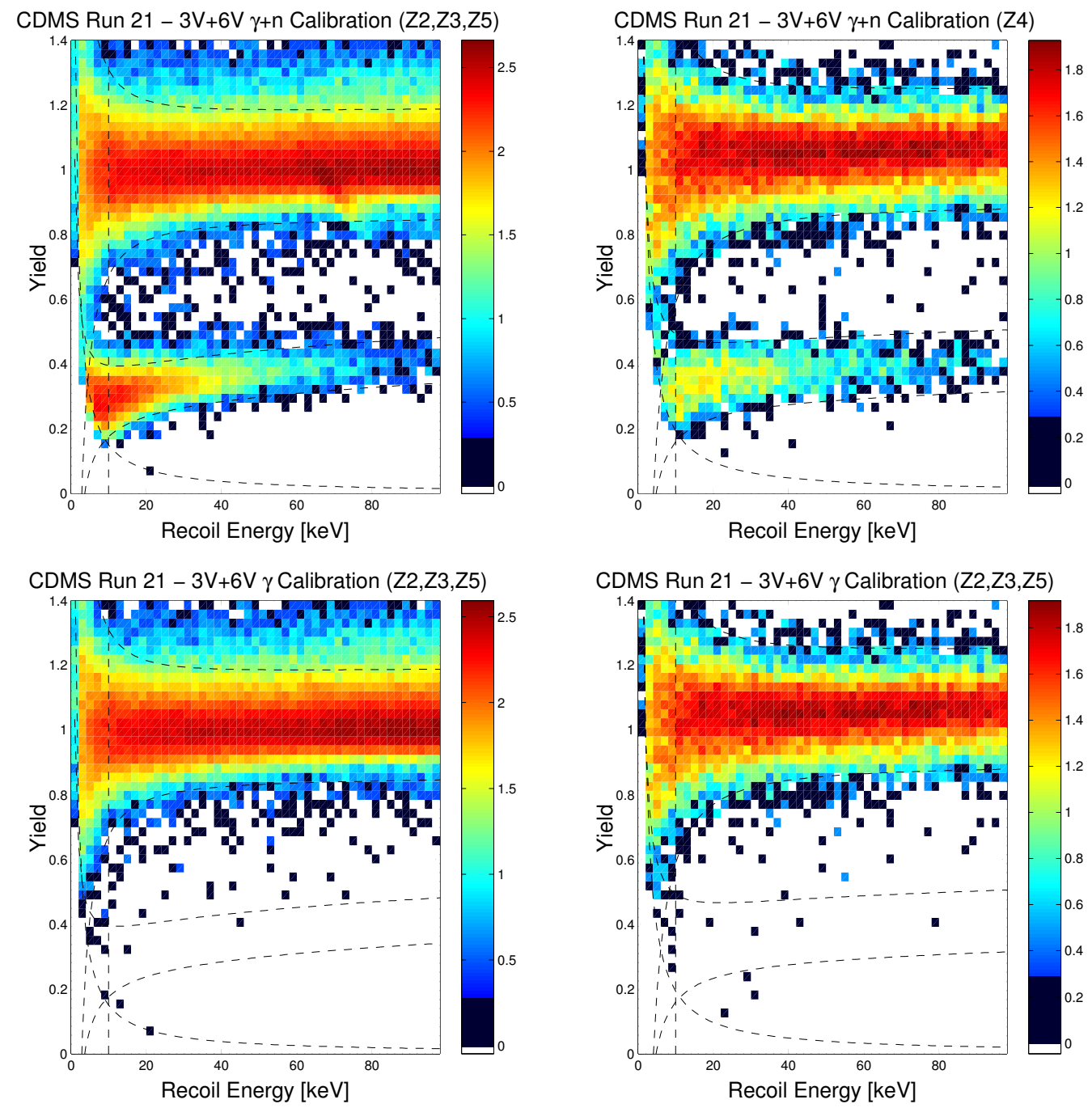

Figure 6.10: 2-D Histograms of Yield vs. Energy in the Calibration Data. The top two graphs are the combined gamma and neutron calibrations to show the separation of the bands and the bottom two graphs are the gamma calibration alone to show the gamma background rejection in the signal region. The left two graphs are for the three inner germanium detectors (Z2, Z3, and Z5) while the right two graphs are for the inner silicon detector (Z4). The color scale is logarithmic with white boxes containing no events and dark blue boxes containing one event only. 


\section{Works Cited}

[1] T. Saab. Search for Weakly Interacting Massive Particles with the Cryogenic Dark Matter Search Experiment. PhD thesis, Stanford University, August 2002.

[2] D.S. Akerib et al. New results from the cryogenic dark matter search experiment. Physical Review D, 68, October 2002.

[3] B. Neganov and V. Trofimov. USSR patent no. 1037771. 1985.

[4] P.N. Luke. Journal of Applied Physics, 64:6858, 1988.

[5] Sunil Ramanlal Golwala. Exclusion Limits on the WIMP-Nucleon ElasticScattering Cross Section from the Cryogenic Dark Matter Search. PhD thesis, The University of California at Berkeley, Fall 2000.

[6] W.H. Press, S.A. Teukolsky, W.T. Vetterling, and B.P. Flannery. Numerical Recipes in C: The Art of Scientific Computing. Cambridge University Press, 1992.

[7] B. Cabrera. Calculation of the phonon energy \& risetime corrections based on a 3d non-matrix approach. CDMS Run 21 ebook \#109, January 2002.

[8] D.D. Driscoll, Jr. Strange behaviour in q-outer. CDMS Run 21 ebook \#283, February 2003.

[9] R.W. Ogburn. Parameterizing the box plot. CDMS Run 21 ebook \#291, April 2003. 


\section{Chapter 7}

\section{Analysis of Beta Contamination}

We have better than $99.99 \%$ rejection of gamma backgrounds using the yield param-

eter $y=\frac{q}{p}$ (the ratio of the ionization signal to the phonon signal). A more troubling source of contamination is a beta emitter. In the energy range of interest, betas have a short penetration length such that they preferentially show up as surface events. Our detectors have a thin dead layer such that events near the surface of the detector can lose some of their ionization signal into the charge contacts. This results in a lower yield, causing these events to leak down from the electron recoil band into the nuclear recoil band, mimicking a candidate event. We have developed a method using the phonon pulse risetime as a third parameter, along with energy and yield, for assessing the probability that an event is a beta. This method allows us to reject surface events from our WIMP candidate list in our science-running data (we refer to this data as our "low-background" dataset to differentiate it from data taken with calibration sources present).

One of the primary motivations in performing this analysis is discriminating against beta-induced events in the subset of multiple-scatter events. In particular, multiple-scatter nuclear recoils are used to directly measure our neutron background. WIMPs have such a low probability of interacting at all that a WIMP multiple scatter is virtually impossible. Any multiple scatters in the nuclear recoil band are caused by neutrons and not WIMPs. We use this measurement of multiple-scatters to make a statistical subtraction of our neutron background from our final list of WIMP candidates.

In a study of the multiple-scatters in the previously-analyzed portion of this data [1], we found that only the triple-scatters and non-nearest-neighbor double-scatters showed a clear separation between electron recoil and nuclear recoil events. The nearest-neighbor double-scatters did not have a well-defined population of nuclear recoil events due to the presence of a population of beta-beta double-scatters. Any of these betas which contaminate our measurement of the neutron background will cause 
us to overestimate that background, and hence underestimate the number of our final nuclear recoil candidates which could be WIMPs. Therefore, we must severely limit the possibility of contamination by beta events in order to be able to use the NND events in our statistical subtraction.

Eliminating the nearest-neighbor doubles (NNDs) in the previous analysis left only 3 multiple-scatter events, such that our measurement of the neutron background was dominated by our statistical uncertainty. Monte Carlo simulations [2] indicate that including the NND-scatters, when combined with a two-fold increase in the amount of data from the previous analysis, should increase number of multiple-scatter events by a factor of four, significantly above the statistics-limited threshold.

\subsection{Selecting a Sample Population of Betas}

One problem we have in modeling the betas is selecting a sample of events with which to define and test a discrimination parameter. Unlike our other calibration sources, we cannot externally introduce a beta source for the very reason they are a troubling contamination - the electrons are not penetrating and cannot enter the cryostat. We also cannot risk a separate calibration run using an internal beta source like ${ }^{109} \mathrm{Cd}$ because we may actually contaminate the detectors in the process. A previous run contained a detector contaminated with ${ }^{14} \mathrm{C}$. Z6, which was also in that run, shows evidence of a beta-emitting surface contamination. Z6 was placed as one of the outer detectors for this reason.

One way of selecting a beta sample is to use nearest-neighbor, double-scatter events (NND, events which occur in two adjacent detectors) in the gamma calibration set. These events have a high proportion of betas from events in which the gamma interacts near the surface in one detector and ejects a electron which travels across to the neighboring detector. We can select for these events using the yield as in Figure 7.1 where we have defined a "beta" as any event with a yield less than the lower cutoff for electron recoil events. Monte Carlo simulations estimate the rate of background neutrons in the ${ }^{60} \mathrm{Co}$ dataset on the order of 1 in 100,000 [3], so we include events with yields all the way down to the charge threshold.

Nuclear recoils also populate this area of the yield-energy plane in the low-background data, so we must have some other means of identifying betas in addition to the yield. We are not really interested in rejecting betas per se, rather we are interested in rejecting any surface events with incomplete charge collection (low yield). The current model of CDMS detectors are called ZIPs because they are Z-sensitive, that is, we can use information from the shape of the phonon pulse to determine its rough position along the z-axis (in cylindrical coordinates). This allows us to discriminate events in the bulk from events on the top and bottom surfaces of the detectors. 


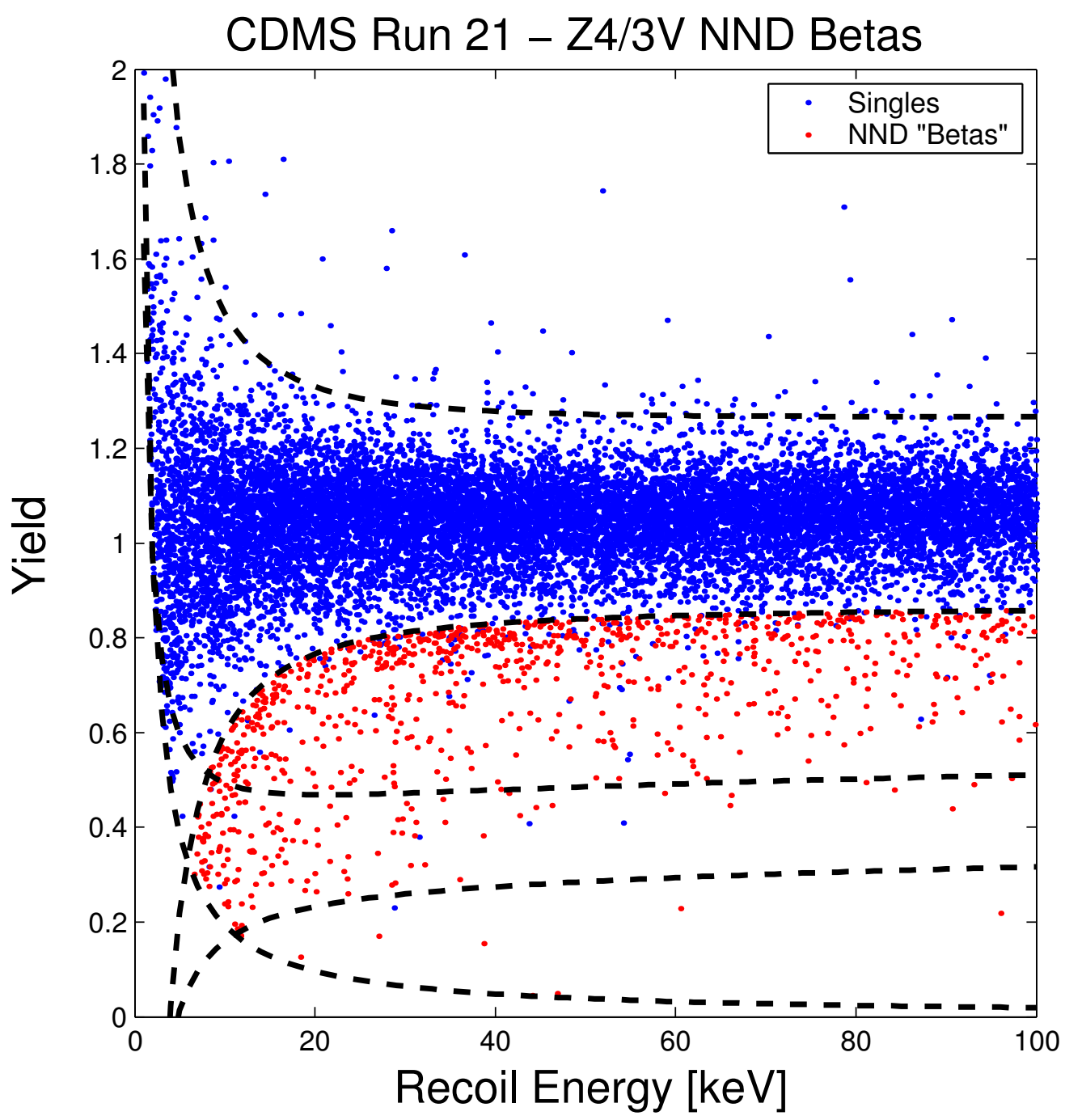

Figure 7.1: Nearest-Neighbor Double-Scatter Betas at Low-Yield. A sample plot of yield-vs.-energy is shown for the silicon detector Z4 (3V bias) where the blue points are single-scatters from the gamma calibration and the red points are low-yield, nearestneighbor double-scatters from the gamma calibration. This plot demonstrates the leakage of these NND "betas" into the signal region, the nuclear recoil band. 


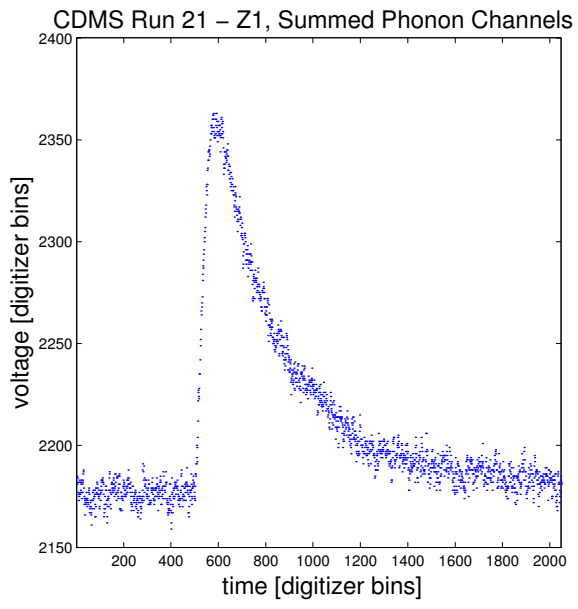

Figure 7.2: A Sample Phonon Pulse. Individual datapoints are shown, indicating that we have information about the risetime of the pulse.

\subsection{Risetime as an Indicator of Depth}

The main surface discrimination parameter we have is the risetime of the phonon pulse. It is a well-documented effect (see e.g. [4]) that an event which occurs close to a surface deposits its phonon energy faster than an event in the bulk. Since we record the rising edge of our pulses, then we are able to use the risetime to rule out surface events. The example pulse shown in Figure 7.2 shows that we have information about the risetime, where the individual data points digitized on the leading edge are shown.

Figure 7.3 shows a sample plot of risetime vs. yield for three populations of events: single-scatters from the gamma calibration, single-scatters from the neutron calibration, and nearest neighbor double betas from the gamma calibration. The gamma singles make a clear feature at their characteristic yield $\left(y_{0} \sim 1\right)$ and risetime $\left(R T_{0} \sim 12 \mu \mathrm{s}\right.$ for $\mathrm{Ge}$ and $R T_{0} \sim 6 \mu \mathrm{s}$ for $\mathrm{Si}$ ) as do the neutrons with similar risetimes and $y_{0} \sim 0.3$. The betas leak out of the gamma band as a rough sketch of the physics might predict. For yields near the gamma band, which represent events in the bulk, the risetime profile has the same shape as the gammas. As the yield decreases, so does the risetime, as you would expect from surface events (low risetime) with poor charge collection (low yield). The population of events also decreases with decreasing yield as fewer events deposit large portions of their ionization signal in the electrode.

Previous analyses used a flat cut in the risetime-yield plane (as shown by the black horizontal line in the Figure 7.3). Figure 7.4 shows the data fraction of both the NND 


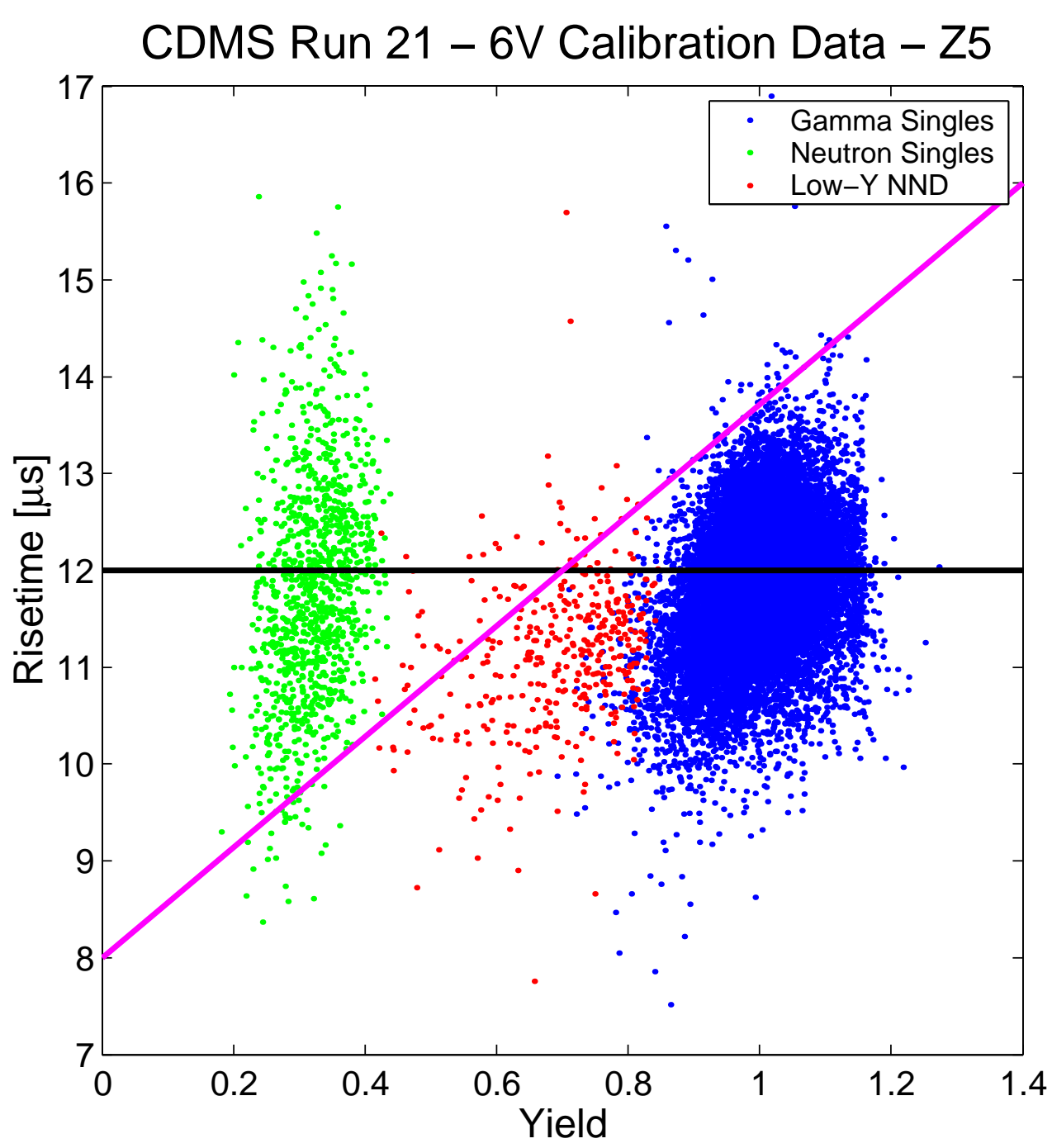

Figure 7.3: Risetime-vs.-yield plot for calibration data. The data shown are for the germanium detector $\mathrm{Z} 5$ at $6 \mathrm{~V}$ bias. Risetime vs. yield is plotted for three populations of events: single-scatters from the gamma calibration in blue, single-scatters from the neutron calibration in green, and low-yield, nearest-neighbor double-scatters (NND) from the gamma calibration in red. The NND events are used as a sample population of "betas." 


\begin{tabular}{|l|r|r|r|r|}
\hline \multirow{2}{*}{$\begin{array}{l}\text { Detector } \\
\text { (material) }\end{array}$} & \multicolumn{2}{|c|}{ Cal. Neutron Singles } & \multicolumn{2}{c|}{ Cal. NND Betas } \\
\cline { 2 - 5 } & Data Frac. & Total No. & Data Frac. & Total No. \\
\hline Z1 $(\mathrm{Ge})$ & $47.60 \%$ & 2061 & $5.61 \%$ & 107 \\
\hline Z2 $(\mathrm{Ge})$ & $49.00 \%$ & 1645 & $7.96 \%$ & 226 \\
\hline Z3 $(\mathrm{Ge})$ & $43.54 \%$ & 1346 & $9.88 \%$ & 253 \\
\hline Z4 $(\mathrm{Si})$ & $64.25 \%$ & 1530 & $15.41 \%$ & 318 \\
\hline Z5 $(\mathrm{Ge})$ & $42.70 \%$ & 1068 & $10.71 \%$ & 392 \\
\hline Z6 $(\mathrm{Si})$ & $64.89 \%$ & 1538 & $20.53 \%$ & 341 \\
\hline
\end{tabular}

Table 7.1: Data fraction of neutrons and betas surviving a risetime cut. The population of neutrons comes from anti-coincident single-scatters in the neutron calibration and the population of betas comes from anti-coincident NND events in the gamma calibration. The risetime levels were taken from the previous analysis [1], 12 $\mu s$ for the germanium detectors and $6 \mu s$ for the silicon detectors. Total number is the number of events in the dataset before a risetime cut.

betas and the calibration neutrons which are above a certain level of risetime in the $6 \mathrm{~V}$ data. Table 7.2 shows the data fraction surviving the cut for the levels chosen for the previous analysis of the $3 \mathrm{~V}$ data only, where we require $R T>12 \mu \mathrm{s}$ for germanium and $R T>6 \mu \mathrm{s}$ for silicon. Using the same levels as in the previous analysis, we cut between $80 \%$ and $95 \%$ of the betas while keeping more than $40 \%$ of the neutrons. We could perhaps do better by making a yield-dependent cut as suggested by the magenta diagonal line, but a statistical description of the data should provide a more complete characterization.

\subsection{Maximum Likelihood Fit for the Betas}

In the previous chapter, we parameterized the distribution of gammas and the distribution of neutrons as a Gaussian in yield with a mean and width which varied with energy. Our acceptance band was defined by hard limits in that parameter space which chose a certain percentage of events enclosed by those limits. We now extend that method by including the risetime and characterizing the distribution of betas. First, we exclude the electron recoil band in the yield-vs.-energy plane to reject the photon background. In the remaining parameter space, we fit the NND events from the gamma calibration to a distribution function for the betas and the single-scatters from the neutron calibration to a distribution function for the neutrons. We then compute the relative probability that an event is a beta or a neutron given that 

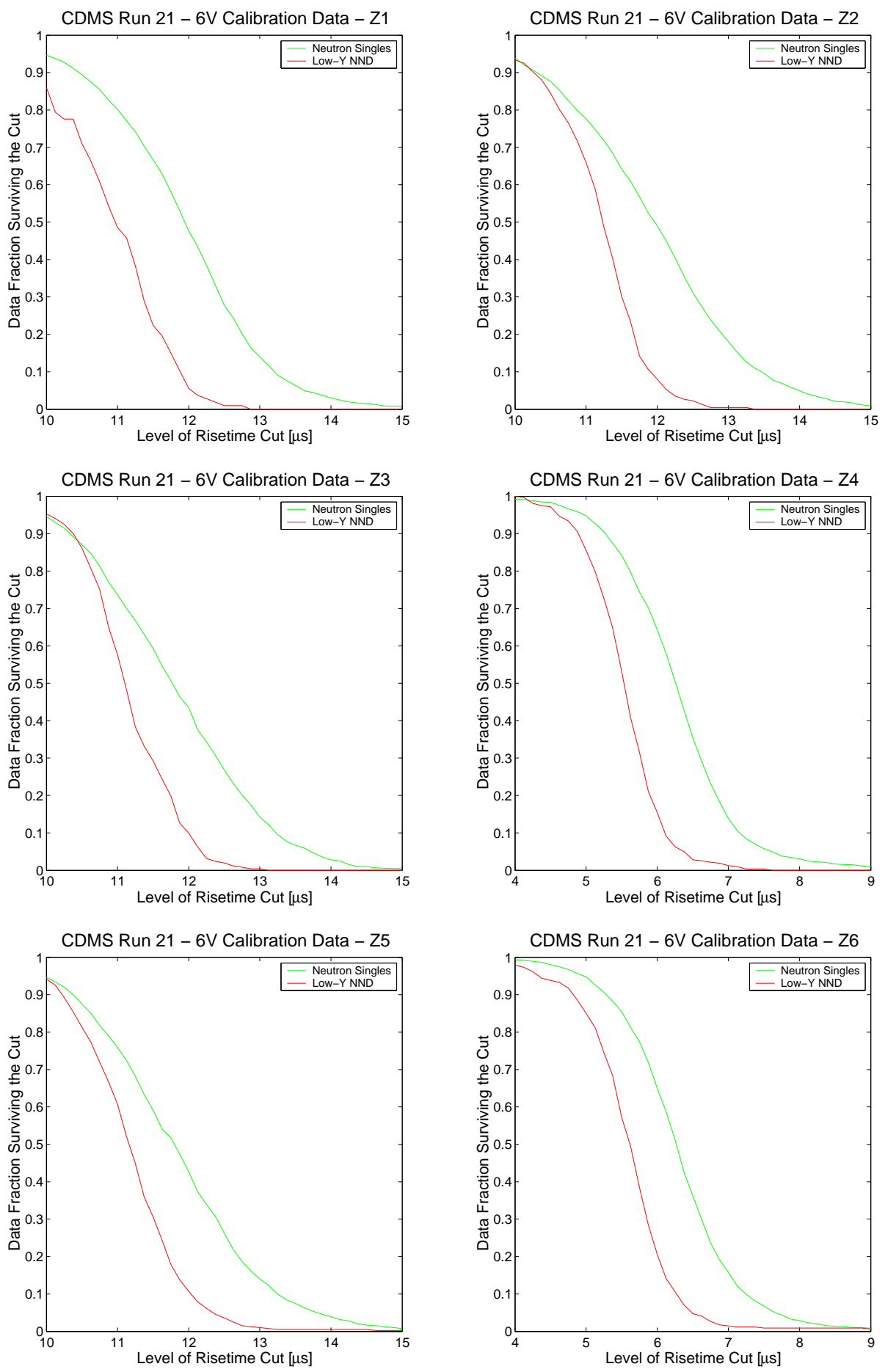

Figure 7.4: Data fraction of neutrons (green) and betas (red) surviving a risetime cut. The population of neutrons comes from veto anti-coincident single-scatters in the neutron calibration and the population of betas comes from veto anti-coincident NND events in the gamma calibration. Note the different scales for the risetime in the germanium detectors (Z1,Z2,Z3,Z5) compared with the silicon detectors $(\mathrm{Z} 4, \mathrm{Z} 6)$. 
event's risetime, yield, and energy and choose a cut level based on the ratio of these probabilities which preferentially rejects betas.

The original suggestion for this method came from Bernard Sadoulet and much of its initial development is the work of Vuk Mandic [5]. After some trial and error with the calibration data, we see that in slices of energy the distribution in the risetime-yield plane has the same, roughly triangular shape seen in Figure 7.3, although the exact size and location of the feature may be different. Through mostly empirical methods, we model the distribution of betas as a Gaussian in risetime and an exponential in yield:

$$
\begin{array}{r}
\mathcal{L}_{\beta}(y, r ; \theta)=\frac{g(y, r ; \theta)}{N(\theta)}=\left(\frac{1}{N(\theta)}\right) e^{\frac{y}{\tau}} e^{-\frac{\left(r-r^{\prime}(y)\right)^{2}}{2 \sigma}} \\
r^{\prime}(y) \equiv A y+B=A\left(y-y_{0}\right)+r_{0} \\
N(\theta) \equiv \int_{\mathrm{y}_{\min }}^{\mathrm{y}_{\max }} d y \int_{-\infty}^{+\infty} d r g(y, r ; \theta)=\sqrt{2 \pi} \sigma \tau\left(e^{\frac{\mathrm{y}_{\max }}{\tau}}-e^{\frac{\mathrm{y}_{\min }}{\tau}}\right)
\end{array}
$$

where the likelihood function $\left(\mathcal{L}_{\beta}\right)$ is a function of the yield $(y)$, the risetime $(r)$, and three free parameters $(\theta), \tau, A$, and $\sigma$. The function $r^{\prime}(y)$ describes the mean of the Gaussian in risetime, which is a function of the yield. We have fixed the parameter $B$ by requiring the line of $r^{\prime}(y)$ to pass through the center of the gamma population $\left(y_{0}, r_{0}\right)$. We have also fixed the parameter $\sigma_{r}$ to match the width of the gamma population in risetime. $N(\theta)$ is a constant required to normalize the total probability $\left(\int \mathcal{L}_{\beta}(y, r ; \theta)=1\right)$ over the range of interest. The range of risetimes does not really extend over the range $(-\infty,+\infty)$, but the width of the Gaussian is much smaller than the mean $\sigma_{r} \ll r^{\prime}(y)$, so this is a good approximation which significantly simplifies $N(\theta)$ and integrals of $\mathcal{L}_{\beta}$.

This choice of likelihood function, while empirical, was informed by a conceptual understanding of the physics discussed above. First, we have assumed a correlation between risetime and yield, represented by $r^{\prime}(y)$, which comes from their dependence on the depth of the interaction. For any given yield, there exists a corresponding risetime which then has a Gaussian shape from the uncertainty of the measurement. The mean of the Gaussian $r^{\prime}(y)$ was chosen to be linear for simplicity and should be a good approximation to the true (unknown) distribution over the narrow range of $y$ between the bands. The distribution of yields which corresponds to the distribution of event depths was then assumed to vary along that line acting as the height of the Gaussian in risetime. The gammas, which are fully penetrating for most energies, are dominated by events in the bulk and have a characteristic risetime and yield $\left(r_{0}\right.$ and $\left.y_{0}\right)$. The line of $r^{\prime}(y)$ is fixed so that it passes through this point, which should be well-defined. The betas have lower penetration depths at lower yield, so it is not unreasonable to assume that the amplitude should falls off away from this point. 
Again, the overall assumption that we are making is that the shape of the distribution is the same for different energies, although the free parameters are be functions of energy. This should be a reasonable assumption since the risetime and the yield are both functions of the penetration depth. We make separate fits for each detector at each bias voltage since their distributions may be different due to variations in detector response.

The first step is to divide our NND data up into energy bins and perform a maximum likelihood fit to the parameters which describe the shape in the risetimeyield plane. The bottom of the electron recoil band and the charge threshold both vary with energy. We make a flat boundary in yield at the top and bottom of the energy slice so that the distribution in the risetime-yield plane is not influenced by the cut that we use (See Figure 7.5 for an example.), and we have taken all risetimes. No restrictions were placed on the energy, yield, or risetime in the second detector. The energy/yield regions are shown in Figure 7.5. We have limited the top of the box by the bottom of the $3 \sigma$ gamma band and we have let the bottom of the box go all the way to the charge-energy threshold.

We could estimate the errors on the parameters by the covariance matrix:

$$
V_{k l}^{-1}=\sum_{i}-\frac{\partial^{2} \ln \mathcal{L}\left(y_{i}, r_{i} ; \tau, A, \sigma\right)}{\partial \theta_{k} \partial \theta_{l}}
$$

as suggested in [6]. Unfortunately, "For finite samples, however, [this equation] can result in an underestimate of the variances" [6]. For some energy bins in some datasets, there were only a few events and the uncertainties were far smaller than the small sample size would indicate. A better method is to use

$$
\ln \mathcal{L}\left(\theta^{\prime}\right)=\ln \mathcal{L}_{\text {max }}-\Delta \ln \mathcal{L}
$$

to make contours of confidence. The extreme limits of those contours define the uncertainties $\Delta \ln \mathcal{L} \sim \frac{\sigma^{2}}{2}$ for a one-parameter fit and values of $\Delta \ln \mathcal{L}$ prescribed in [6] for multiple parameters and different confidence levels. These are still not exact confidence levels, but should do a better job than merely taking the curvature at the maximal point for approximating the uncertainties when we are doing the energy fits.

We then perform a fit to each parameter to make the parameters smoothly varying functions of energy,

$$
\begin{array}{r}
\tau(E ; \theta)=a_{\tau} E^{b_{\tau}} \\
A(E ; \theta)=a_{A} E^{b_{A}} \\
\sigma(E ; \theta)=\sqrt{c^{2}+\frac{d^{2}}{E^{2}}}
\end{array}
$$




\section{CDMS Run $21-6 V^{60}$ Co Cal. data - Z5}

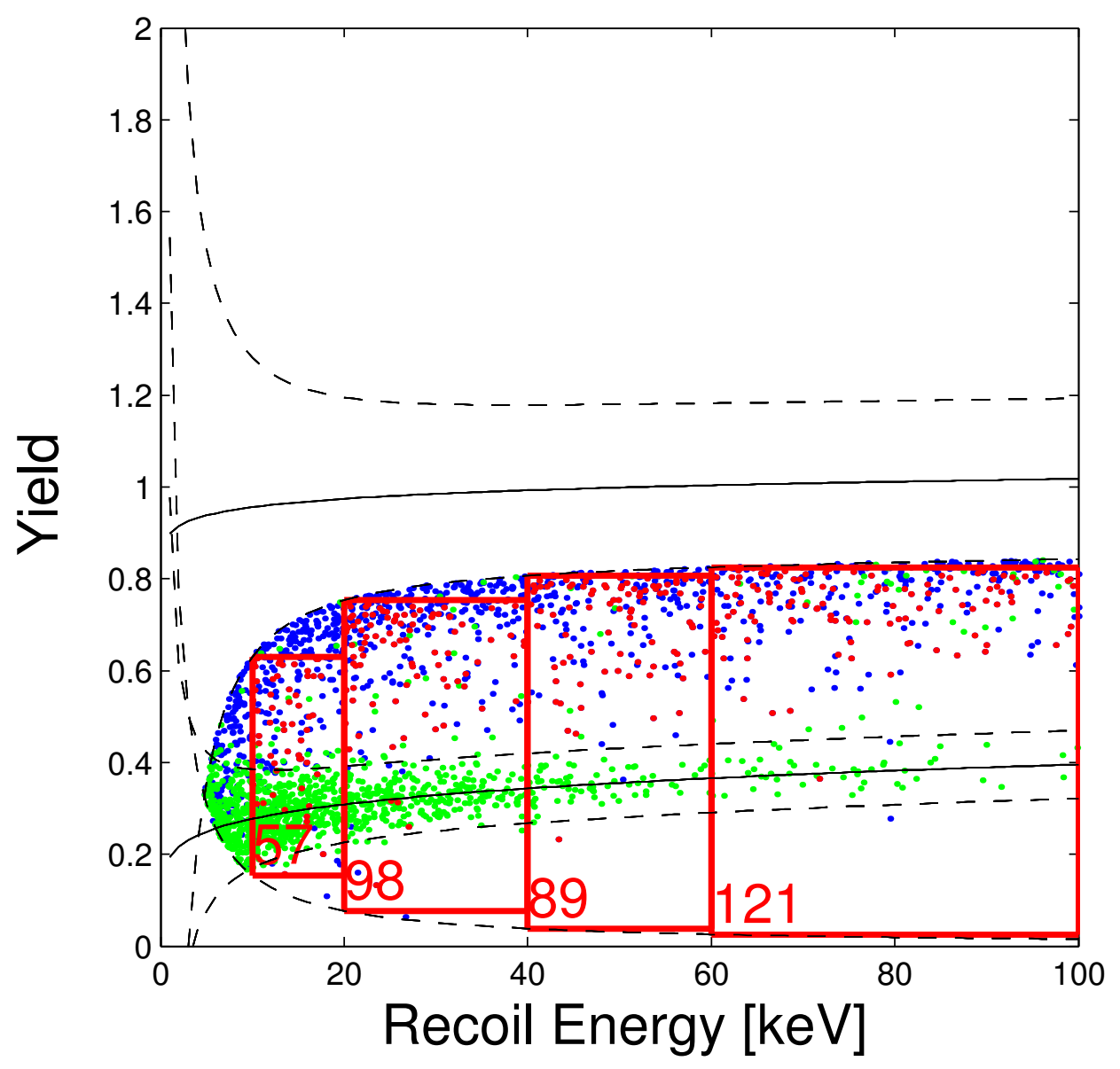

Figure 7.5: Energy Band Regions for the Beta Fits in Detector Z5 (Ge). The blue events are single-scatters in the gamma calibration, the green events are single-scatters in the neutron calibration, and the red events are nearest-neighbor double-scatters in the gamma calibration. The regions are chosen to be rectangular so as not to bias the yield distribution (see text). The upper limit in yield is defined by the bottom of the electron recoil band and the lower limit in yield is defined by the charge threshold. 


\section{CDMS Run $21-6 \mathrm{~V}^{60}$ Co Cal. data - Z5}

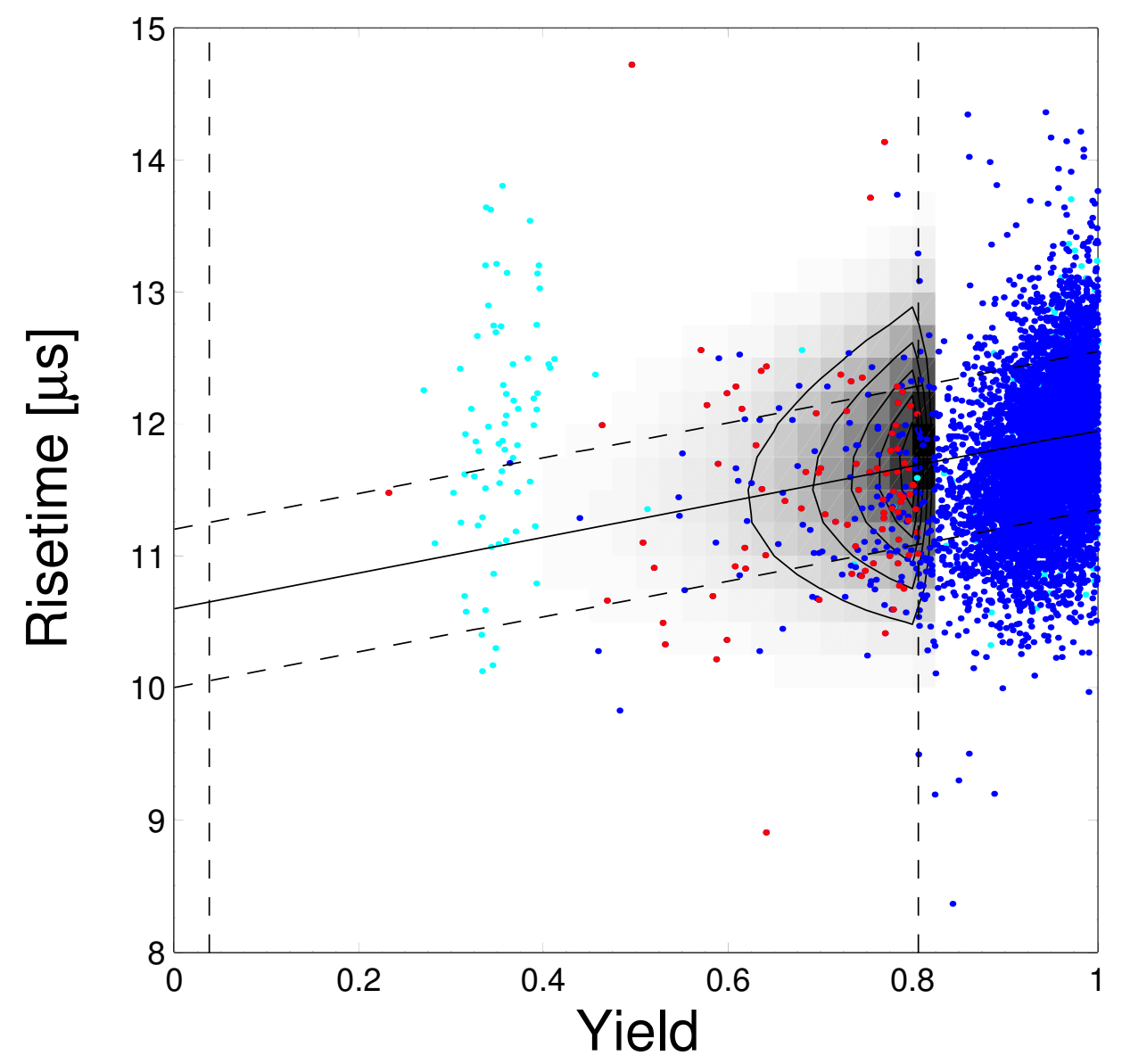

Figure 7.6: A Sample Likelihood Fit for Betas in the Risetime-Yield Plane for Detector Z5 (Ge), from 40-60 keV. The blue events are single-scatters in the gamma calibration, the green events are single-scatters in the neutron calibration, and the red events are nearest-neighbor double-scatters in the gamma calibration. Both the grey scale as well as the contours are intended as a indication of the shape of the beta model for this energy band. The vertical dashed lines represent the bounds of the yield from Figure 7.5. The solid diagonal line represents the center of the risetime Gaussian in Equation 7.1, constrained to go through the center of the gamma singles, and the accompanying dashed lines represent the $1 \sigma$ width of that Gaussian. 
where we have chosen the $\tau$ and $\sigma$ functions to have the same form as those used for the yield-vs.-energy bands.

We construct a similar distribution function for the neutrons, which is a twodimensional Gaussian in the risetime-yield plane for slices of energy:

$$
\begin{array}{r}
\mathcal{L}_{n}(y, r ; \theta)=\frac{g(y, r ; \theta)}{N(\theta)}=\left(\frac{1}{N(\theta)}\right) e^{-\frac{\left(y-y_{g}\right)^{2}}{2 \sigma_{y}}} e^{-\frac{\left(r-r_{g}\right)^{2}}{2 \sigma_{r}}} \\
N(\theta) \equiv \int_{\mathrm{y}_{\min }}^{\mathrm{y}_{\max }} d y \int_{-\infty}^{+\infty} d r g(y, r ; \theta)=\pi \sigma_{y} \sigma_{r}\left[\left.\operatorname{Erf}\left(\frac{y-y_{g}}{\sqrt{2} \sigma_{y}}\right)\right|_{\mathrm{y}=\mathrm{y}_{\min }} ^{\mathrm{y}=\mathrm{y}_{\max }}\right.
\end{array}
$$

where we have made the approximation to integrate over all risetimes as before. The range of the rectangular slice in the yield-vs.-energy plane $\left(y_{\min }\right.$ to $\left.y_{\max }\right)$ was chosen to approximately cover the $\pm 3 \sigma$ nuclear recoil band to include most of the neutrons while limiting contamination from electron recoils (see Figure 7.7).

Our discrimination parameter is be the ratio $\mathcal{R}(y, r \mid E)=\log _{10}\left(\frac{\mathcal{L}_{\beta}(y, r \mid E)}{\mathcal{L}_{n}(y, r \mid E)}\right)$. Figure 7.9 shows a sample distribution of $\mathcal{R}$ in the yield-vs.-energy plane with an integration over risetime, with separate plots of the distribution of betas and the distribution of neutrons. We see that $\mathcal{R}$ is dominated by the neutron function except at low energies, essentially reproducing the band structure developed in the previous chapter. More instructive is Figure 7.10, which shows the distribution for a high risetime and a low risetime.

\subsection{Elimination of Betas in the NND Neutrons}

As mentioned above, nearest-neighbor double-scatters in the previous analysis [1] did not show a clear separation between gamma-gamma scatters and neutron-neutron scatters. Two methods were attempted to allow the nearest-neighbor doubles to be used in the neutron background measurement, which would reduce the statistical errors in the subtraction of the neutron background by increasing the acceptance for multiple-scatter nuclear recoils. A risetime cut in either one or both of the detectors was shown to reduce the background of betas in the double scatters, but at the cost of some of our neutron double scatters. As shown in Table 7.1, this cut removes approximately $\sim 90 \%$ of the betas and keeps $\sim 50 \%$ of the neutrons in each detector. Also, a cut on the separation between the position of the two hits preferentially eliminated betas, which should be surface-to-surface events with small angular separation [7]. Figure 7.11 shows the angular separation for the two types of events with a cut on the nuclear recoil band. A cut at $\sim 30^{\circ}$ (0.5 radians) removes $95 \%$ of the betas while keeping $50 \%$ of neutrons. Table 7.2 shows the approximate data fraction of betas and neutrons which survive the four combinations of these cuts. 


\section{CDMS Run $21-6 \mathrm{~V}{ }^{252} \mathrm{Cf}$ Cal. data $-\mathrm{Z5}$}

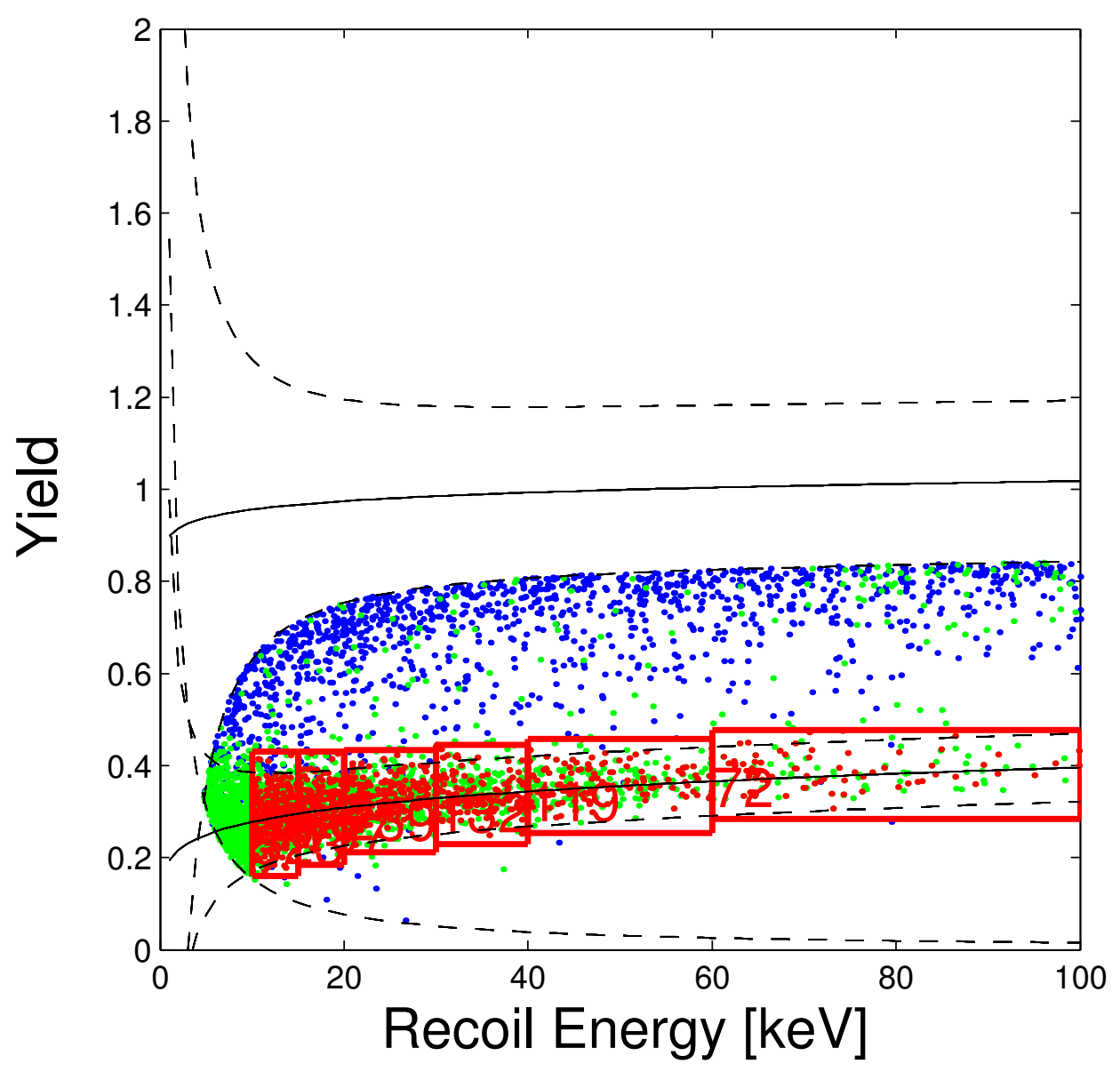

Figure 7.7: Energy Band Regions for Detector Z5 (Ge). The blue events are singlescatters in the gamma calibration, the green events are single-scatters in the neutron calibration, and the red events are nearest-neighbor double-scatters in the gamma calibration. The regions are chosen to be rectangular so as not to bias the yield distribution (see text). The limits in yield are chosen roughly to cover the $3 \sigma$ nuclear recoil band. 


\section{CDMS Run $21-6 V^{252} \mathrm{Cf}$ Cal. data $-\mathrm{Z5}$}

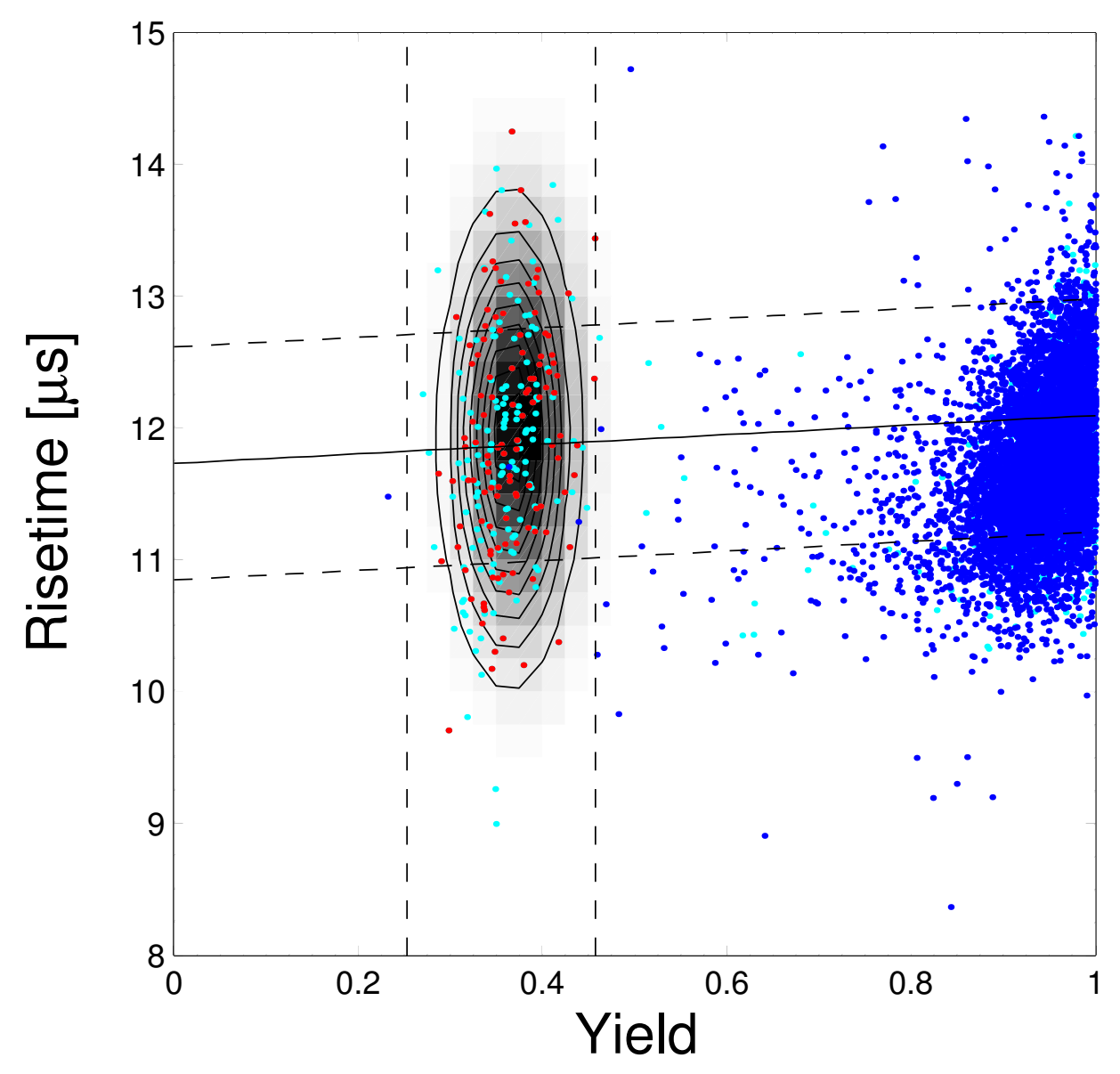

Figure 7.8: A Sample Likelihood Fit for Neutrons in the Risetime-Yield Plane for Detector Z5 (Ge), from 40-60 keV. The blue events are single-scatters in the gamma calibration, the green events are single-scatters in the neutron calibration, and the red events are chosen neutron singles for the fit. Both the grey scale as well as the contours are intended as a indication of the shape of the neutron model for this energy band, which is a two-dimensional Gaussian. The vertical dashed lines represent the bounds of the yield from Figure 7.7 . 
CDMS Run 21 - 3V Likelihood Ratio $\log _{10}(\mathrm{Lb} / \mathrm{Ln})-\mathrm{Z4}$
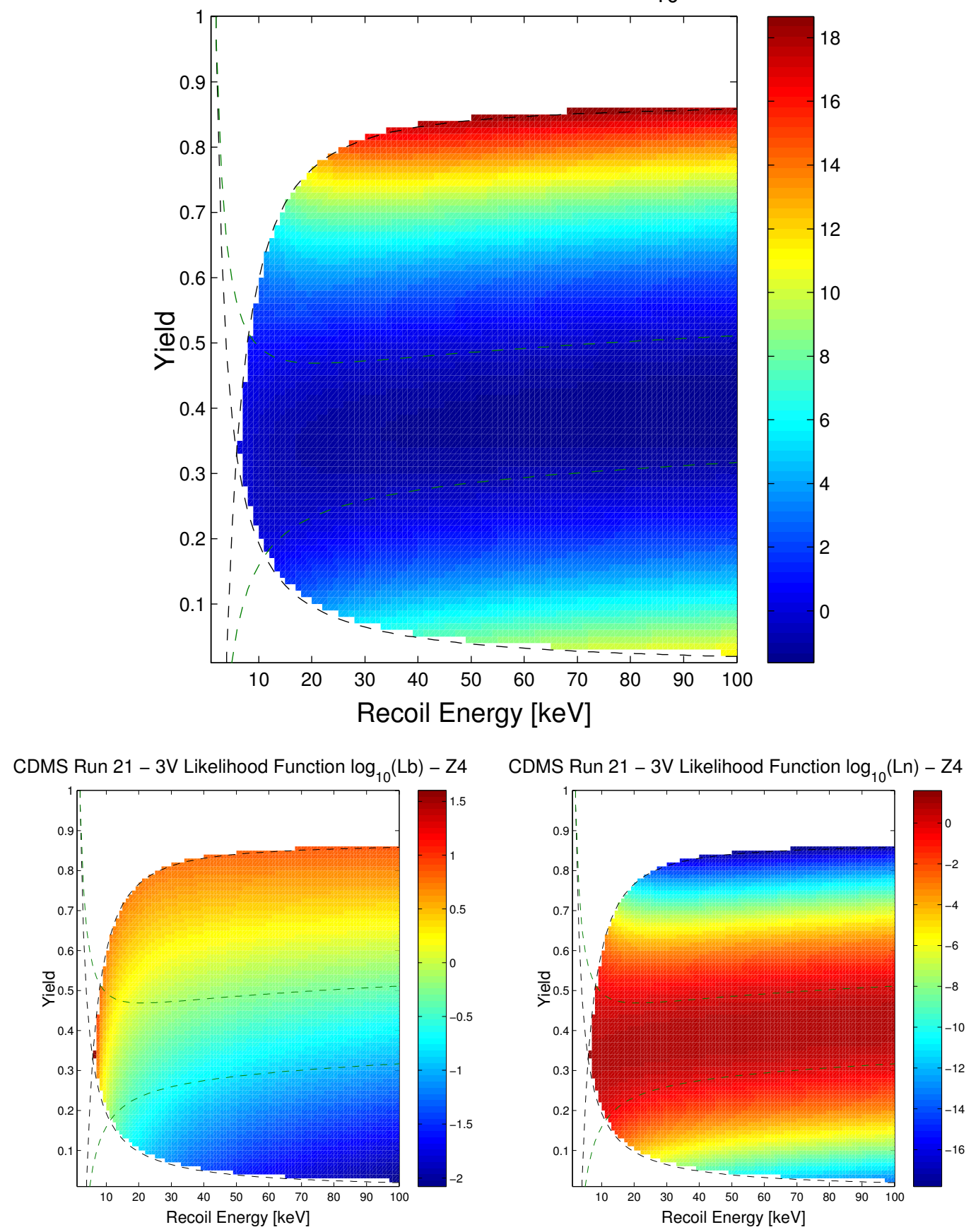

Figure 7.9: Distribution of the Discrimination Parameter $\mathcal{R}$ Integrated Over Risetime. The distributions are for the silicon detector Z4. The upper graph shows the (log) ratio of the beta likelihood to the neutron likelihood functions. The bottom graphs show the beta part alone (left) and the neutron part alone (right). 

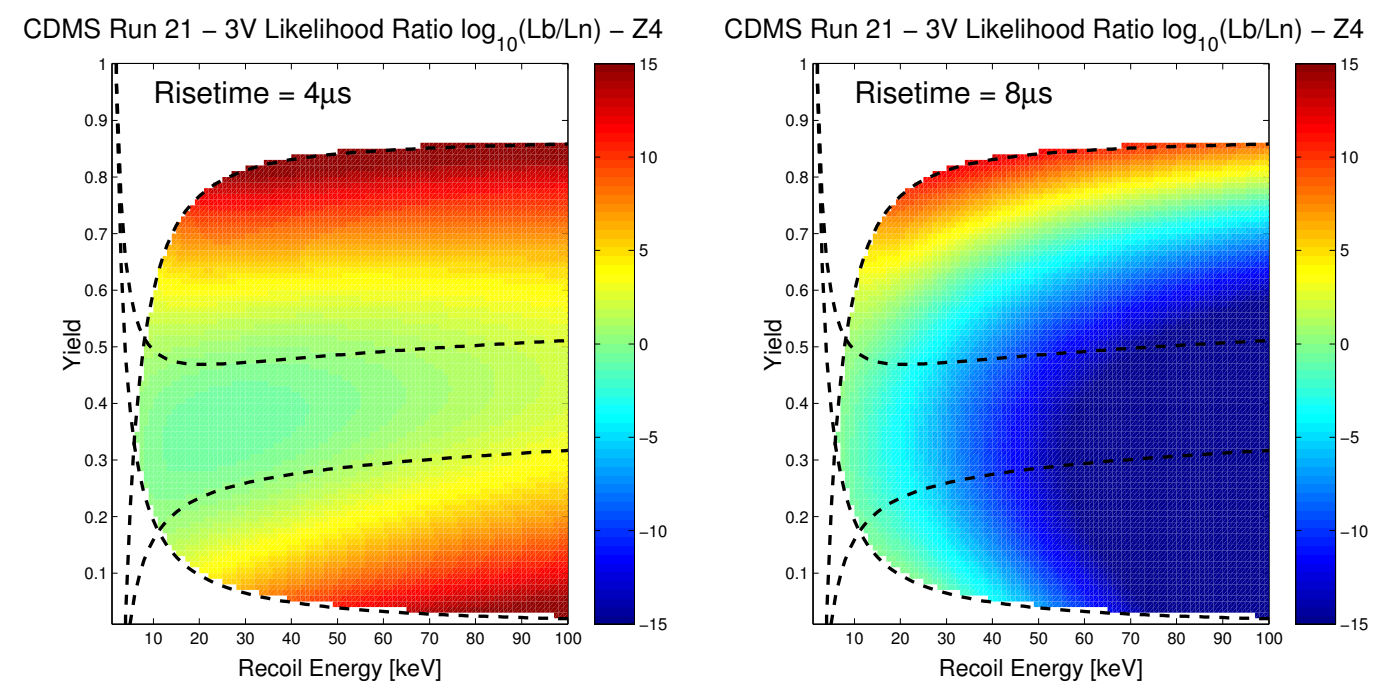

Figure 7.10: Distribution of the Discrimination Parameter $\mathcal{R}$ at High and Low Risetimes. The distributions are for the silicon detector Z4, with a characteristic risetime of $6 \mu \mathrm{s}$. The false color plot represents the likelihood function and both graphs are shown with the same colormap. At high risetimes (right), the likelihood ratio is dominated by the nuclear recoil band, while at low risetimes (left), the beta function dominates.

\begin{tabular}{|l|l|r|r|}
\hline Position cut & Risetime cut & $\begin{array}{r}\text { Data Fraction } \\
\text { for Neutrons }\end{array}$ & $\begin{array}{r}\text { Data Fraction } \\
\text { for Betas }\end{array}$ \\
\hline None & 1 event passes & $75 \%$ & $36 \%$ \\
\hline None & Both events pass & $25 \%$ & $4 \%$ \\
\hline Azimuth & 1 event passes & $38 \%$ & $2.5 \%$ \\
\hline Azimuth & Both events pass & $13 \%$ & $0.25 \%$ \\
\hline
\end{tabular}

Table 7.2: The estimated data fraction surviving combinations of a position cut and a risetime cut after a Nuclear Recoil Band Cut [8]. The survival fractions are approximations based on the rough survival percentages of each cut separately. 


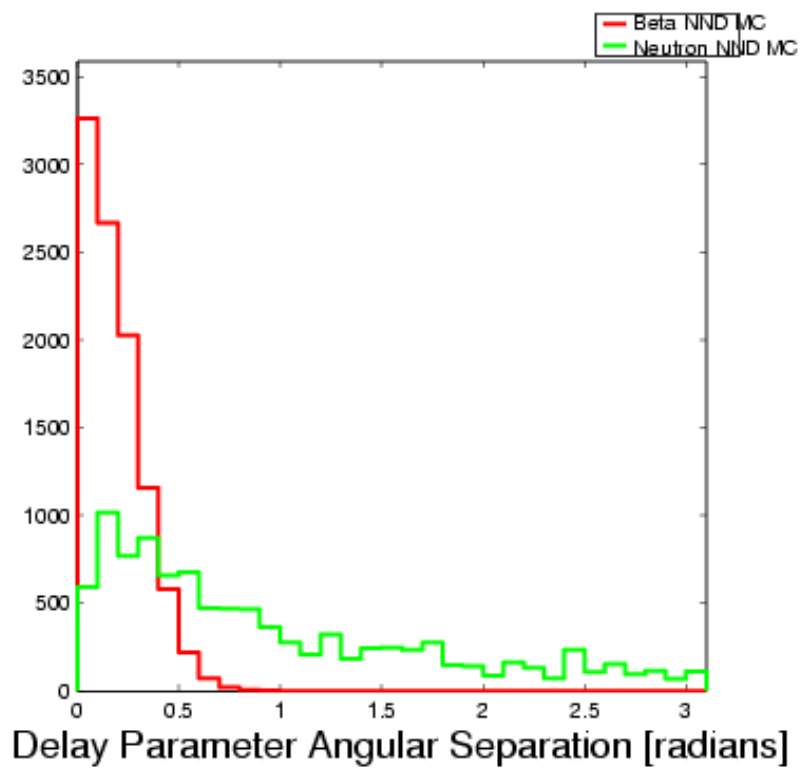

Figure 7.11: Angular Separation of NND Betas and Neutrons. Nearest-neighbor doubles from the gamma calibration (our "beta" sample) are plotted in red and NNDs from the neutron calibration are plotted in green. 
The eventual decision for the previous analysis was to exclude the NND neutrons altogether because a simple combination of these cuts could not unambiguously eliminate the betas while still keeping a significant number of neutrons. The likelihood distributions developed above allow us to develop a single cut which simultaneously includes the same information in these three separate cuts, allowing us to make a much finer cut in the phase-space of risetime, yield, energy, and separation than we could by looking at each quantity by itself.

In order to use $\mathcal{R}$ as a discrimination parameter, we need to establish the distributions of betas and neutrons in this parameter. Our goal is to establish a cut level such that we can entirely eliminate beta events from the NND events in the low-background population. We cannot analytically determine the distribution of $\mathcal{R}$ since it is a complicated function of three variables $(y, r, E)$, so we use the models of betas and neutrons to make sample populations on which to base our cuts. One concern is that the population of betas that we are using to make our models (NNDs in the gamma calibration) is different than the population of betas that we are trying to exclude in the low-background data. In addition to best-fit model of the betas, we make a conservative model with which to set our cut level such that we do not underestimate the beta leakage. Finally, we can compare these models with our calibration data to test the consistency of our fits and to estimate the efficiency of our cuts before applying them to the low-background data.

We simulated sample populations of 100,000 of events of each type listed below based on the distribution functions in each detector and bias voltage. We created a total of five populations that we are interested in modeling, two sets of neutrons and three sets of betas. Since the distribution functions we have constructed are actually $\mathcal{L}(y, r \mid E)$ where the yield and risetime distributions are functions of energy, we need to provide each set with an energy spectrum so that we can produce triplets of $(y, r, E)$ for each event. The following list describes the energy spectrum used in each of the models:

1. Calibration neutrons - We took the energy spectrum for this population by randomly selecting from the energies of the nearest-neighbor doubles in the neutron calibration data. This set is a subset of the one used to make the fits in that this set includes only the events which had energies from 10-100 keV in both detectors and both events were below the gamma band. This set was created with the purpose of testing the efficiency of our cut levels, and we are interested in more accurately modeling the distribution of events in the $(y, r, E)$ triplet rather than having higher statistics to model the risetime-vs.-yield shape as in the fits.

2. Calibration betas - Again, we used the energy spectrum of the nearest-neighbor 
doubles in the gamma calibration with the same restrictions as in the calibration neutron model.

3. Low-background neutrons - The energy spectrum used here was taken from a Monte Carlo simulation of the neutron background [2].

4. Low-background betas - We used the energy spectrum of low-yield NND events in the low-background dataset since this is the population we are looking to exclude. There is some contamination of this set by neutrons, but it is dominated by the betas when taken over the whole low-yield region from the bottom of the gamma band down to the energy threshold.

5. Conservative low-background betas - This set was constructed based on the lowbackground betas, but with some of the fit parameters altered to increase the number of events with high risetime and low yield. This conservative population was constructed in order to set the cut level such that we would be overestimating the beta leakage into our signal region. The details of this alteration follow.

The two parameters adjusted to make a conservative model based on the best fit model constructed in the previous section are the half-width, half-maximum of the Gaussian in the risetime profile, $\sigma_{r}^{+}$(for $r>r^{\prime}(y)$ ), and the exponential constant in the yield profile, $\tau_{y}$ (see Equation 7.9p. Increasing $\sigma_{r}^{+}$increased the number of betas which have high risetime, thus mimicking a neutron. Increasing $\tau_{y}$ similarly increases the number of betas which leak into the nuclear recoil region. Figure 7.12 shows the number of betas which pass the risetime cut $(r>12 \mu s$ for Ge and $r>6 \mu s$ for $\mathrm{Si})$ and fall in the nuclear recoil region in the parameter space $\left(f_{y}, f_{r}\right)$ :

$$
\begin{array}{r}
\tau_{y}^{\prime}=\left(1+f_{y}\right) \tau_{y} \\
{\sigma_{r}^{+}}^{\prime}=\left(1+f_{r}\right) \sigma_{r}^{+}
\end{array}
$$

The false-color plot represents the percent leakage into the signal region where the "X" at $(0,0)$ represents the original best fit model. The contours shown are the $1 \sigma$ and $2 \sigma$ levels based on the adjusted likelihood function $\mathcal{L}_{\beta}^{\prime}\left(y(E), r(E) \mid \Theta(E), \tau_{y}^{\prime}, \sigma_{r}^{+^{\prime}}\right)$ summed over the actual NND betas in the gamma calibration. We chose our conservative model as the one which maximizes the leakage into the signal region while remaining a $1 \sigma$ fit to the calibration data, as represented by the "O" on the graph. A sample comparison of the data, the best fit model, and the adjusted model, are shown in Figure 7.13 .

We used these models to determine the distribution of $\mathcal{R}$ of betas and of neutrons for each detector and voltage bias. Figure 7.15 shows the distribution of the calibra- 


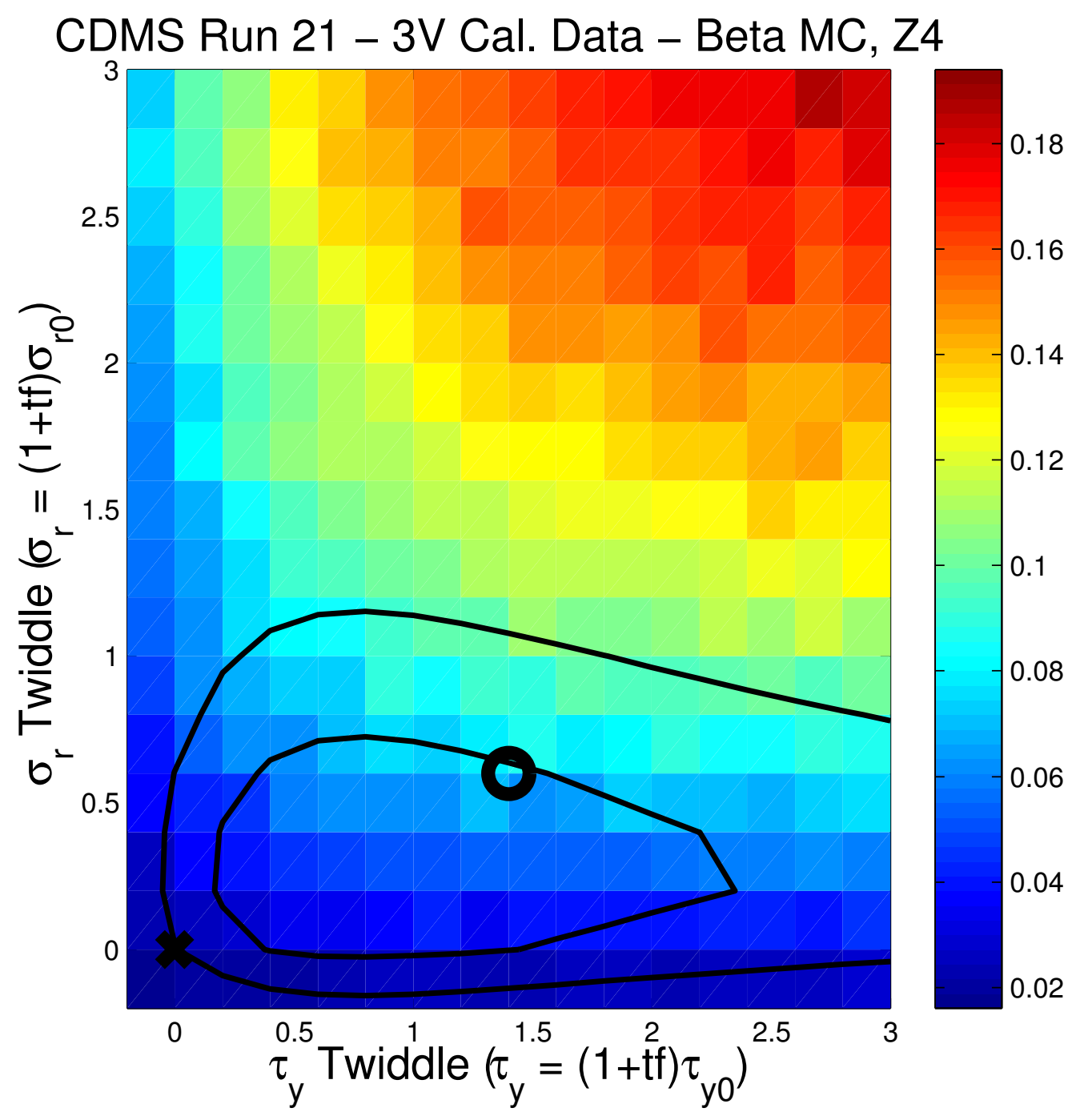

Figure 7.12: Parameter Space for Constructing a Conservative Beta Model, shown for the silicon detector Z4. The $\mathrm{x}$-axis represents the adjustment of the exponential constant in the yield part of the modeled distribution of betas and the y-axis represents the adjustment of the width of the Gaussian in the risetime part of the distribution. The false color plot shows the leakage of betas into the signal region, which increases as the low-yield tail and the high-risetime tail increase (as expected). The contour shows the $1 \sigma$ and $2 \sigma$ regions of a maximum likelihood fit of the model given by $\left(\tau_{y}^{\prime}, \sigma_{r}^{+\prime}\right)$. The ' $\mathrm{X}$ ' at $(0,0)$ is the location of the best-fit model and the ' 0 ' is the point within the $1 \sigma$ contour which maximizes the leakage. 


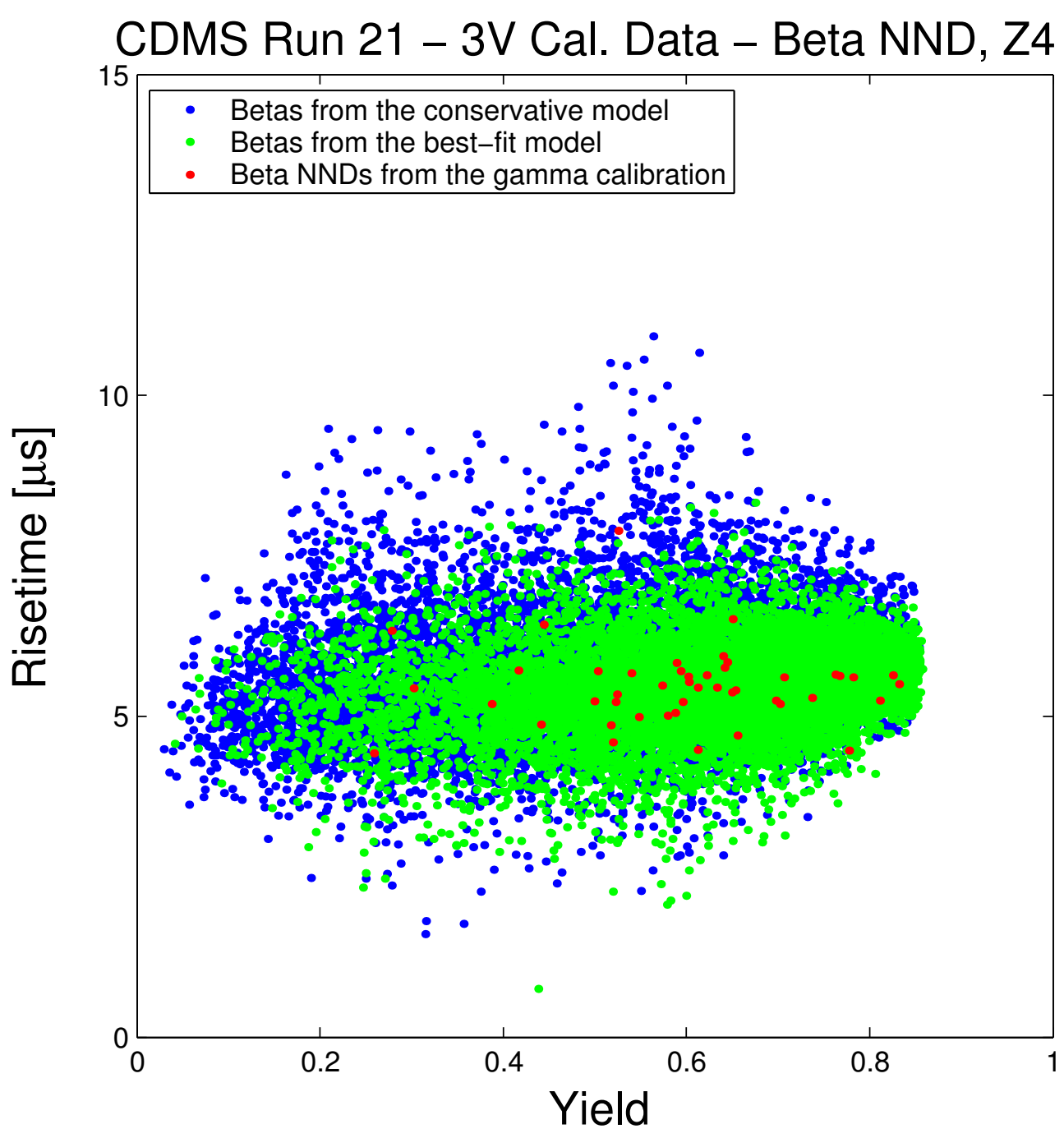

Figure 7.13: Risetime-vs.-Yield Distribution of the Conservative Beta Model, shown for the silicon detector Z4. The NND data are in red, the best-fit model in green, and the conservative model in blue. The horizontal dashed line is the location of the flat risetime cut and the vertical dashed lines are approximate bounds on the nuclear recoil band. The conservative model has more events in the nuclear recoil region and above the risetime cut, indicating a larger leakage into the signal region. 


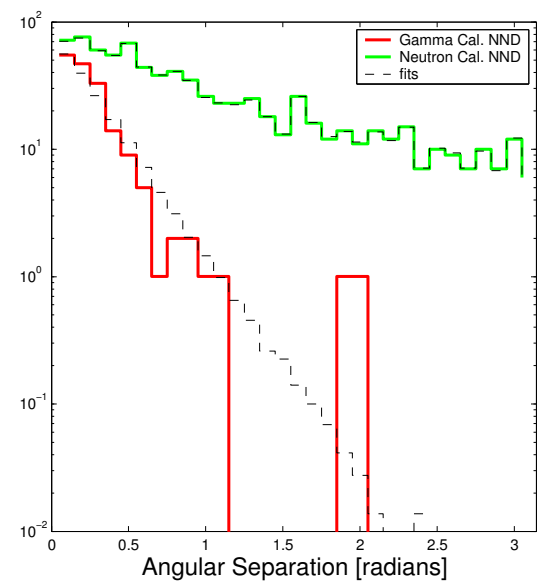

Figure 7.14: A Model of Distribution of Angular Separations. The histograms are shown here in log-scale to emphasize the tails of the distributions. The nearestneighbor double-scatters (NNDs) from the gamma calibration data are shown in red and the NNDs from the neutron calibration are in green. The dashed line represent a randomly-chosen sample based on a model of the distribution. The neutrons are chosen by randomly selecting angular separations from the actual data set. The betas are modeled by a Gaussian centered on zero with an exponential tail at high separations. This model was chosen to allow for high-separation betas for which the statistics in the actual data are low.

tion data for detectors Z4 and Z5 when biased at three volts with the conservative, low-background model included since this is the model on which the cuts are based.

We combine pairs of these distributions in order to create a distribution for the NND events. This assumes that the $(y, r, E)$ triplet for the hit in one detector is uncorrelated with the triplet for the hit in the neighboring detector. We also include the angular separation of the two hits, as shown in Figure 7.11. We must also model this angular separation parameter based on this figure. For the neutrons, we chose a random separation from the actual distribution of NND events in the neutron calibration. The betas have such a sharp cutoff at high angular separation that we don't have enough statistics there to use this same technique. We model the distribution of angular separations in the betas as a Gaussian centered on zero with a exponential tail:

$$
\frac{d N}{d \theta}=A e^{-\theta^{2} / 2 \sigma^{2}}+B e^{-\theta / \tau}
$$

The distributions of the data and of the models are shown in Figure 7.14.

We make the NND distribution of $\mathcal{R}_{i j}$ (for $i-j=1$ ) by combining a pair of 


\begin{tabular}{|r|c|c|c|}
\hline Detector Pair & $3 \mathrm{~V}$ & $6 \mathrm{~V}$ & $3 \mathrm{~V}+6 \mathrm{~V}$ \\
\hline Z1/Z2 & 9 & 7 & 16 \\
\hline Z2/Z3 & 9 & 3 & 12 \\
\hline Z3/Z4 & 15 & 7 & 22 \\
\hline Z4/Z5 & 23 & 11 & 34 \\
\hline Z5/Z6 & 21 & 21 & 42 \\
\hline Total & 77 & 49 & 126 \\
\hline
\end{tabular}

Table 7.3: The Number of Low-Yield, Nearest-Neighbor Double-Scatters in the LowBackground Data.

single-detector models $\left(\mathcal{R}_{i}\right.$ and $\left.\mathcal{R}_{j}\right)$ with an angular separation model $\mathcal{R}_{\theta}$ as:

$$
N_{\kappa}\left(\mathcal{R}_{i j}\right)=\left[N_{\alpha}\left(\mathcal{R}_{i}\right)+N_{\beta}\left(\mathcal{R}_{j}\right)+N_{\delta}\left(\mathcal{R}_{\theta}\right)\right] \delta[\kappa-(\alpha+\beta+\gamma)]
$$

where $N_{x}(\mathcal{R})$ represents the $x^{\text {th }}$ bin of a histogram of $\mathcal{R}$ and the delta function selects the bin where $\mathcal{R}_{i j}=\mathcal{R}_{i}+\mathcal{R}_{j}+\mathcal{R}_{\theta}$. Figure 7.15 shows the histograms for the two individual detectors as well as the combined histogram (including position information).

We use these histograms to construct a cumulative distribution function (CDF) for both neutrons and betas at a particular value of $\mathcal{R}_{N N D}$ such that if we set a cut level at that point, we can estimate the neutron and beta survival rates. Our target is to eliminate all of the betas from our NND population, so we count the total number of low-y NND events in the background data for each detector pair (Table 7.3) and set the cut level for each detector pair such that there is an approximate $1 \%$ chance of a leaked beta-beta NND in our whole low-background dataset. Figure 7.16 shows a sample CDF, now including the low-background data, which is very similar to the calibration data. Table 7.4 shows the cut levels with the expected and actual neutron survival percentages for the calibration data.

Averaging over detector pair and voltage (weighted by the number of low-yield NNDs in Table 7.3), we find a total expected neutron survival percentage of $45.3 \%$ with an actual survival percentage of $38.6 \%$ with a $95 \%$ confidence level range of $(34.8 \%-42.5 \%)$. We are slightly overestimating our neutron survival, but not by too much. None of the 172 NND "betas" (low-yield events) in our gamma calibration survive the cut.

Based on the estimates from Table 7.4 and the reasonable agreement of our estimates with the calibration data, we decided to include the nearest-neighbor double scatters in our measurement of the neutron background. We exclude the detector pair $\mathrm{Z} 1 / \mathrm{Z} 2$ since the predicted neutron survival percentage is too low due to uncertainties in our fitting of the risetime-yield shape of the betas. The survival rate in the best-fit 

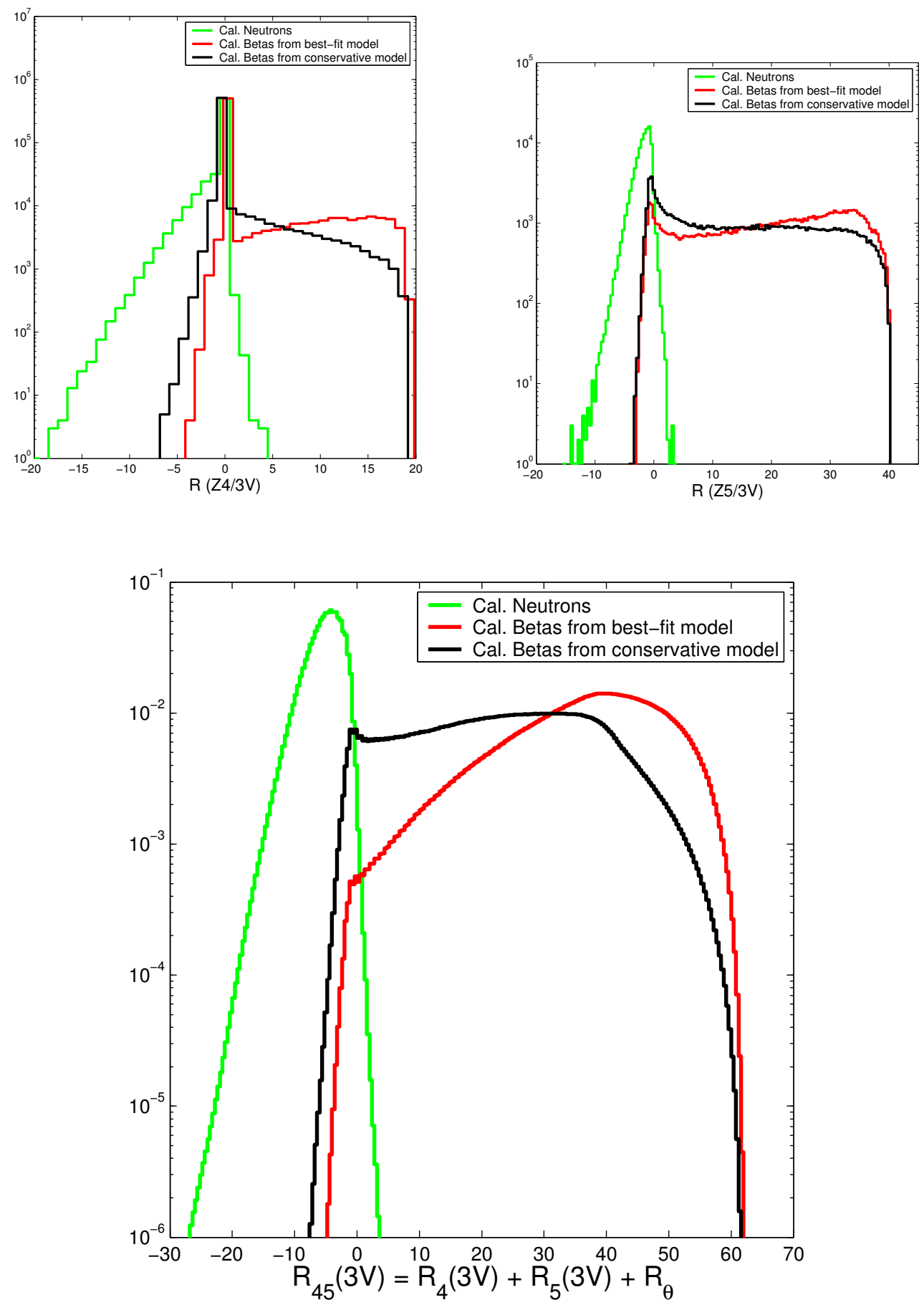

Figure 7.15: Modeled Distribution of the Likelihood Function Ration, $\mathcal{R}$. The top two graphs are the individual distributions for each detector (Z4(Si)-left, Z5(Ge)-right). The bottom graph is the combined distribution including the angular separation information. The neutron model is plotted in green, the best-fit beta model is plotted in red, and the conservative beta model is plotted in black. This model shows good separation between neutrons and betas, while the conservative model is worse. 


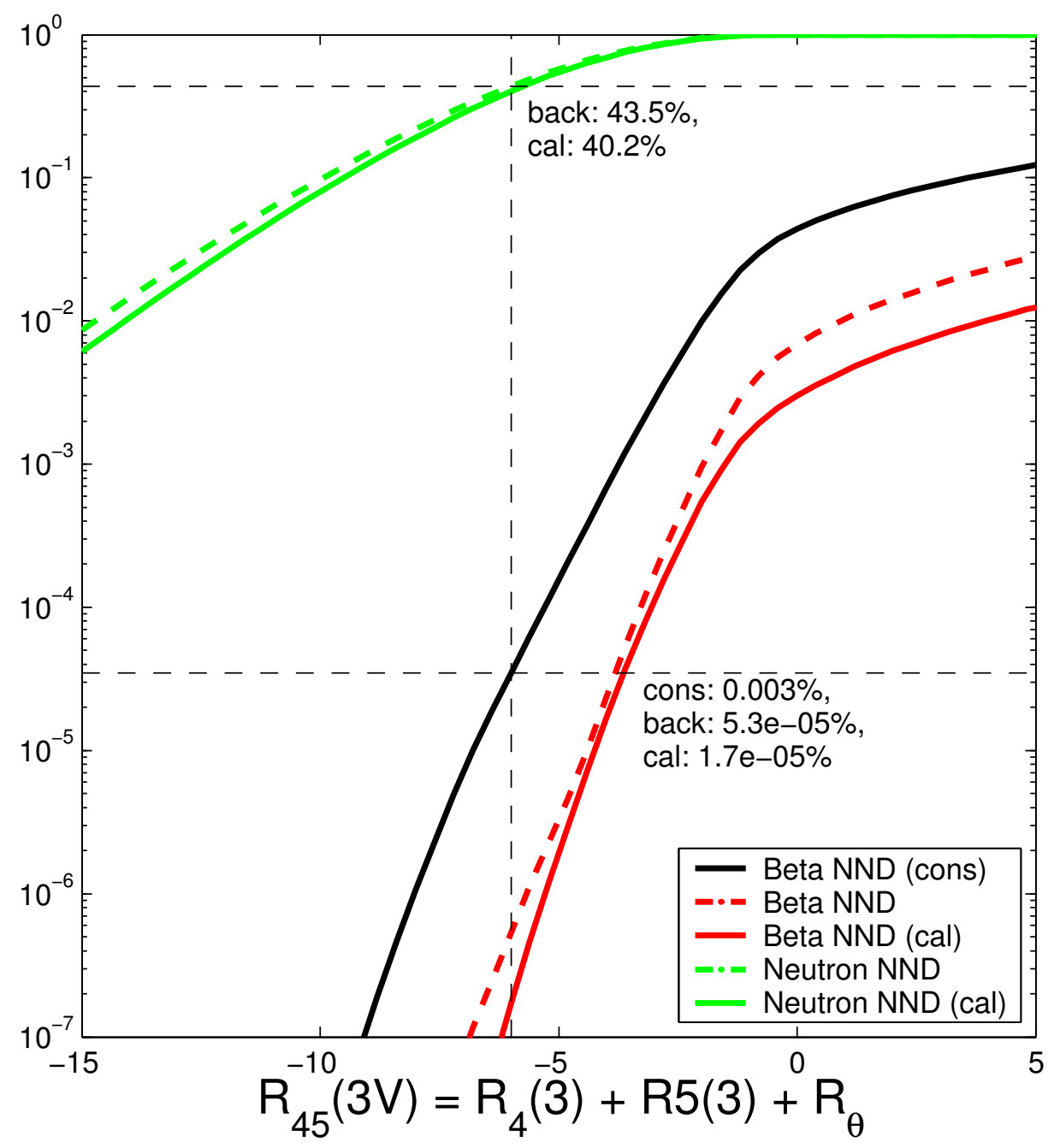

Figure 7.16: Percentage of Events Passing a Cut in $\mathcal{R}$ for the detector pair Z4(Si)/Z5(Ge). The green curves (upper) represent the estimated survival percentage for the neutrons and the red curves (lower) represent the estimated survival percentage for beta. The black curve is the conservative model upon which the cuts are based. The vertical dashed line indicates the location of the cut level for which there is less than a $1 \%$ chance of a leaked event out of the 126 events in the total lowbackground dataset. The horizontal dashed lines are placed at the estimated survival percentage, with the values indicated along that line. 


\begin{tabular}{|r|r|r|r|r|r|r|}
\hline & \multicolumn{7}{l|}{$6 \mathrm{~V}$} \\
\hline $\begin{array}{r}\text { Det. } \\
\text { Pair }\end{array}$ & Cut Level & $\begin{array}{r}\mathrm{n} \text { Survival } \\
(\text { est.) }\end{array}$ & $\begin{array}{r}\text { n Survival } \\
\text { (actual) }\end{array}$ & Cut Level & $\begin{array}{r}\text { n Survival } \\
\text { (est.) }\end{array}$ & $\begin{array}{r}\text { n Survival } \\
\text { (actual) }\end{array}$ \\
\hline Z1/Z2 & -15.6 & $4.0 \%$ & $0 \% \pm 2 \%$ & -32.8 & $0.0 \%$ & $0 \% \pm 4 \%$ \\
\hline Z2/Z3 & -4.4 & $69.6 \%$ & $54 \% \pm 12 \%$ & -7.2 & $42.7 \%$ & $24 \% \pm 8 \%$ \\
\hline Z3/Z4 & -5.2 & $53.0 \%$ & $44 \% \pm 15 \%$ & -7.2 & $28.4 \%$ & $38 \% \pm 16 \%$ \\
\hline Z4/Z5 & -6.0 & $40.2 \%$ & $24 \% \pm 10 \%$ & -6.4 & $45.0 \%$ & $29 \% \pm 12 \%$ \\
\hline Z5/Z6 & -5.2 & $44.8 \%$ & $55 \% \pm 13 \%$ & -5.6 & $41.1 \%$ & $49 \% \pm 9 \%$ \\
\hline
\end{tabular}

Table 7.4: Cut levels and Approximate Survival Percentages for Nearest-Neighbor Double-Scatter Neutrons. Cut levels are shown for each voltage bias and detector pair, with estimated and actual cut levels from the calibration data shown. The $95 \%$ statistical uncertainties on the actual survival percentage shown above are in good agreement for most detector pairs. The systematic errors in our models were not computed. Based on these values, the detector pair Z1/Z2 is excluded from analysis.

model is quite good, but the data do not constrain the conservative model well enough to disallow such a broad distribution. Figure 7.17 shows the predicted distribution of the parameter $\mathcal{R}$ for NNDs compared with the calibration data, demonstrating good agreement between data and prediction. The models were based on this data, so this level of agreement is expected, and the plots are more a confirmation that the method is consistent. The conservative model for the beta, which is more neutron-like than either the best-fit model or the data, was created to ensure that any variations between NND population in the calibration data and in the low-background data do not lead us to under-estimating the beta leakage.

\subsection{Elimination of Betas in the Single-Scatters}

We also use the above characterization to reduce any beta contamination in the lowbackground germanium single-scatters. In the presence of a WIMP signal, we would either have to make a restrictive cut similar to that used on the NND events above to eliminate all of the betas or we would have to use our models to estimate the beta leakage into the signal region. Since we expect to be limited by a background of neutrons in this data taken at our shallow site, we can tolerate some number of betas in our WIMP candidates. Any beta single-scatters which contaminate our final WIMP candidate list will be an overestimate to the number of WIMPS, and hence give us a weaker sensitivity. We still want to limit the beta contamination to optimize the result, though, so we choose a cut level which minimizes the beta leakage

while maximizing our sensitivity to nuclear recoils. We treat the germanium and 

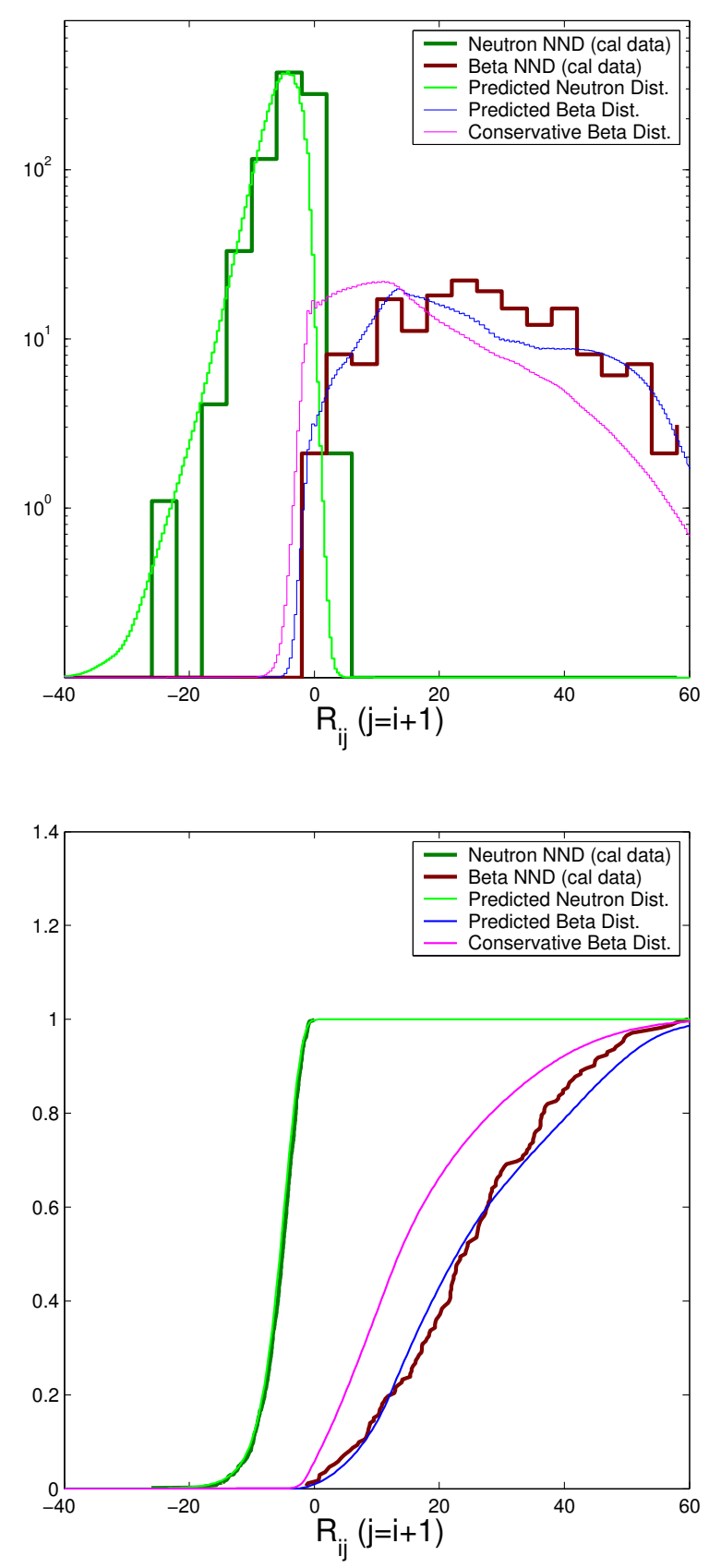

Figure 7.17: Comparison of the Distribution of $\mathcal{R}$ Between Models and Data. The top plot shows good agreement between the calibration data and our models, while the bottom plot indicates that the conservative beta model is more neutron-like than either the best-fit model or the low-yield NNDs in the gamma calibration data. 
silicon detectors differently here since the germanium detectors are used for detecting WIMPs while the silicon detectors are used for measuring the neutron background (see Chapter 2).

For each germanium detector and bias voltage $(d)$, we minimize the quantity:

$$
f=\frac{\mathcal{P}_{90}\left[\sum_{d} \epsilon_{n}(d) N_{n}(d)+\epsilon_{\beta}(d) N_{\beta}(d)\right]}{\sum_{d} \epsilon_{n}(d)}
$$

where $\epsilon_{x}$ represents the data fraction surviving the cut (from the single-detector distributions of $\mathcal{R}$ above) and $\mathcal{P}_{90}$ is the $90 \%$ upper limit of a Poisson process such that $\lim _{x \rightarrow 0} \mathcal{P}_{90}(x)=2.30$, which keeps $\epsilon_{n}>0$. $N_{x}$ represents the number of events of each type of particle. In the previous analysis [9], we estimated a background of approximately 4 neutron single-scatters per detector. In Chapter 8 , we see that we have 9 multiple-scatter events, with a ratio of single-to-multiple-scatters consistent with all of the WIMP candidate events being due to a neutron background. Since we are ultimately interested in subtracting off some of the measured neutron background, the Poisson uncertainty in 9 detected multiple-scatters would imply that $\frac{\sqrt{\left(N_{n}\right)}}{N_{n}}=\frac{3}{9}=$ $33 \%$ of those events are neutrons. We therefore use $N_{n}=0.33 \times 4 \sim 2$ as an estimate of the number of neutrons per detector pair and bias voltage. For high-mass WIMPs, the exact placement of the cut is not critical since the uncertainty in the measurement is dominated by the statistical uncertainty in the measured number of multiple-scatters. We could do a better job for low-mass WIMPs by using a less-restrictive cut since the neutron subtraction doesn't greatly effect the limit in that case. A future analysis will analyze the case of low-mass and high-mass WIMPs separately based on different cut levels in $\mathcal{R}$. Table 7.5 shows the cut levels and the expected survival fraction.

Table 7.5 also shows the cut levels for the two silicon detectors, where the cut was made similarly to the cut for the NND events in the previous section. The net result of this analysis is to exclude the two silicon detectors, Z4 and Z6, as well as the germanium detector Z1. Based on the amount of calibration data we have, we cannot convincingly eliminate a beta background while still keeping an appreciable amount of sensitivity to nuclear recoils. This would indicate that more calibration data should be taken in future runs at our deep underground site, perhaps including a dedicated run with triggering configured specifically for acquiring NND data. 


\begin{tabular}{|r|r|r|r|r|}
\hline & \multicolumn{2}{|l|}{ 3V } & \multicolumn{2}{l|}{} \\
\hline Det. & Cut Level & n Survival & Cut Level & n Survival \\
\hline Z1 $(\mathrm{Ge})$ & -20.0 & $0.2 \%$ & -40.0 & $0.0 \%$ \\
\hline Z2 $(\mathrm{Ge})$ & -2.4 & $60.4 \%$ & -4.0 & $44.4 \%$ \\
\hline Z3 $(\mathrm{Ge})$ & -2.0 & $60.0 \%$ & -3.6 & $36.1 \%$ \\
\hline Z5 $(\mathrm{Ge})$ & -2.4 & $34.7 \%$ & -2.0 & $55.9 \%$ \\
\hline \hline Z4 $(\mathrm{Si})$ & -6.0 & $8.0 \%$ & -7.6 & $7.0 \%$ \\
\hline Z6 $(\mathrm{Si})$ & -10.0 & $0.6 \%$ & -10.0 & $1.1 \%$ \\
\hline
\end{tabular}

Table 7.5: Estimated Neutron Survival Percentage in the Single-Scatter Events. These estimates are based on the modeled distributions of neutrons given a cut on the $(\log )$ ratio of the likelihood that it is a beta vs. a neutron given the simulated event's risetime, yield, and energy. The low survival percentage for the silicon detectors (Z4 \& Z6) as well as the germanium detector Z1 lead us to exclude these detectors from the analysis. 


\section{Works Cited}

[1] D.S. Akerib et al. New results from the cryogenic dark matter search experiment. Physical Review D, 68, October 2002.

[2] Sharmila Kamat. Comparison of the external neutron Monte Carlo simulations to the 6V data. CDMS Internal Note, June 2003.

[3] Sharmila Kamat. Run 21 expected internal/external neutrons background for the calibrations. CDMS Internal Note, January 2003.

[4] R.M. Clarke et al. Enhanced ballistic phonon production for surface events in cryogenic silicon detector. Applied Physics Letters, 76(20):2958-2960, May 2000.

[5] Vuk Mandic. Fitting betas and neutrons. CDMS Run 21 ebook \#228, August 2002.

[6] Particle Data Group. Review of Particle Physics, volume 3. Springer, 1998.

[7] Vuk Mandic. Position dependence of double scatters. CDMS Run 21 ebook \#228, August 2002.

[8] Richard Schnee. Choice of cuts for nearest-neighbor multiples. CDMS Run 21 ebook \#275, January 2003.

[9] T. Saab. Search for Weakly Interacting Massive Particles with the Cryogenic Dark Matter Search Experiment. PhD thesis, Stanford University, August 2002. 


\section{Chapter 8}

\section{Results and Conclusions}

Finally, we assemble a collection of WIMP-candidate events which will either provide a signal above our remaining neutron background or constrain the allowed crosssection/mass parameter space. First, we can make a "no-subtraction" limit based on the spectrum of candidate events which remain after our cuts, including the betaeliminating cut described in the previous chapter. This limit makes no assumptions about the content or presence of any backgrounds. We do expect to have a significant neutron background in our candidate list, but we make no a priori assumptions about the lack or presence of a WIMP signal. We therefore conservatively produce an upper limit on the allowed cross-section for a given mass. However, we have knowledge about the background of neutrons, both from Monte Carlo simulations of known sources as well as direct measurement using multiple scatter events. Therefore, we can use this knowledge to make an improved limit based on a statistical subtraction from our candidate events interpreting some of them as neutrons. In making an upper limit, we must be particularly conservative in making this subtraction since an overestimate of the predicted neutron flux will lead to fewer WIMP candidates in the final limit and hence a stricter bound on the upper limit of the allowed cross-sections.

\subsection{Data Cuts and Their Efficiencies}

The population of single-scatter events in the germanium detectors provide us with our WIMP candidates. We start with a total of $8,733,457$ events taken at two bias voltages $(4,761,644$ at $3 \mathrm{~V}, 3,971,813$ at $6 \mathrm{~V}$ ) with a livetime of 119.1 days (66.6 ld, 52.5 ld). The vast majority of these events are background events, so we make a series of cuts in order to reject as much of this background as possible.

Our experiment is surrounded with an active muon veto (see Chapter 3), whose threshold is set to reject minimum-ionizing muons. We wish to construct a cut which 
excludes events which are coincident within some time window of a muon passing through our experimental volume. The trigger rate for the veto is $5.0 \mathrm{kHz}$ based on the distribution of trigger times in randomly-triggered data as seen in Figure 8.1, so we must also keep this window as small as possible to prevent an unnecessary deadtime associated with muon hits.

An improved veto cut was used for this analysis which takes into account the difficulty in finding the exact time of a pulse near the noise threshold. We make two different cuts depending on the charge energy [1]. Above $3.5 \mathrm{keV}$, the pulsefinding algorithm does a good job of finding the charge pulse above the noise. An event is considered to be muon-coincident if there is a muon-trigger in the window of $[-15 \mu \mathrm{s}, 5 \mu \mathrm{s}]$ around the pulse trigger. $90 \%$ of randomly-triggered data have nearest veto hits outside this window. Similarly, any event which is not caused by a muon has a $10 \%$ chance of being rejected due to an accidental coincidence within this window. Given the above trigger rate, this is close to the expected value of $[\exp (-5.0 \mathrm{kHz} \cdot 15 \mu \mathrm{s})+\exp (-5.0 \mathrm{kHz} \cdot 5 \mu \mathrm{s})] / 2=91 \%$.

Below $3.5 \mathrm{keV}$, the pulse-finding algorithm occasionally finds large noise spikes rather than the pulse itself. At these energies, we use the time of the phonon pulse instead. The pulse-finding algorithm does a better job of finding the phonon pulses at these energies, particularly for nuclear recoils, which have a larger phonon signal than an electron recoil. These phonon-located events have a broader distribution of times, so a slightly wider window of $[-23 \mu s, 15 \mu s]$ is used for these pulses. This broadening results in a slightly lower rejection efficiency for accidental coincidences (82\%). The expected value is $[\exp (-5.0 \mathrm{kHz} \cdot 23 \mu \mathrm{s})+\exp (-5.0 \mathrm{kHz} \cdot 15 \mu \mathrm{s})] / 2=83 \%$.

The efficiency of this cut for rejecting muons can be found by selecting a population of muon-like events, that is, events with more than about $8 \mathrm{MeV}$. Based on these events, our average muon-rejection efficiency is $99.87 \%$ [1]. This efficiency is much lower than the previously-quoted $99.95 \%$ and is caused by a unknown defect in the hardware/analysis chain which mistakenly puts some small class of events at a fixed time relative to the trigger. These events are within the wider $40 \mu s$ window used previously, but are now outside our smaller window. These events do appear to be veto-coincident and no explanation has been found as to why they are being misidentified.

The data quality cuts we use reject unusable or otherwise unreliable events. Two cuts remove data which are not viable as signal events: one removes events which have been tagged as random triggers used for noise characterization and the other removes specific time series which are associated with known operational problems such as power glitches, fridge crashes, earthquakes, etc. Several other cuts are related to the noise. One looks at the standard deviation of the pre-pulse baseline to see if there is pile-up, that is, if the tail of a previous event is leaking into the beginning of this one. A chi-squared cut similarly looks for pile-up or other irregularities by 


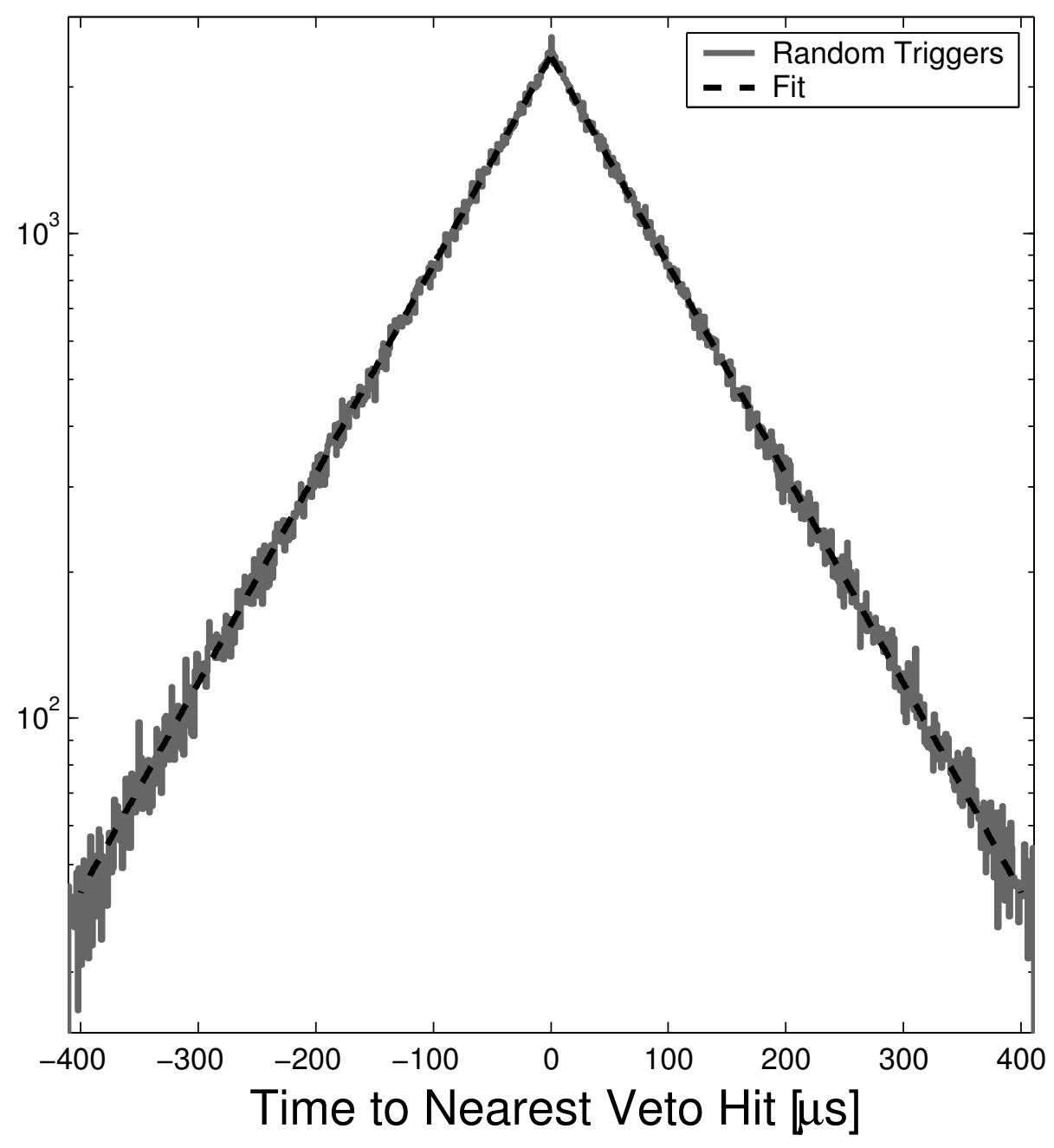

Figure 8.1: The distribution of nearest veto hits relative to random triggers. The slope of the plots on either side of zero gives us the trigger rate. 
comparing the pulse with the template fit. Post-pulse pile-up results in a large value of chi-squared since a two-pulse trace is poorly fit by a one-pulse template. Currently, only the charge channels have chi-squared information.

The remainder of the cuts applied to the low-background data have been described in detail in Chapter 6. A "single," "double," or other "multiple" hit is selected based on the number of hits above the phonon search threshold (Section 6.2). The fiducial volume cut described in Section 6.3 was used to select events in the inner $85 \%$ of the detector ( $85 \%$ of the volume, radially outward from the center). This both shields the detector from external particles and also excludes the outer portion of the charge electrode where the field lines may break down. Finally, events in the electron recoil band are excluded to reject more than $99.9 \%$ of the gamma-like background. It is worthy to note that the beta-rejection cut described in Chapter 7 also rejects a substantial portion of the remaining gammas. There are no low-yield events in the gamma calibration which survive this cut, yielding a 90\% confidence level of $99.999 \%$ rejection of our gamma background.

We compute the efficiencies of our cuts for neutrons based on a Monte Carlo simulation of our external neutron background (the Run 21 Monte Carlo is the work of Sharmila Kamat [2] 3] et al.). The reason for performing this simulation is to account for internal scatters within a single detector. We must keep track of where a scatter occurs since we have two separate charge electrodes - a disk-shaped inner electrode and an annular outer electrode. A neutron can multiply-scatter such that one hit deposits energy in one electrode and then scatters again and deposits energy in the other. There is also a small region between the two electrodes where energy can be shared between the two. Earlier comparisons of the Monte Carlo simulation with our neutron calibration data have produced effective dimensions for the inner and outer regions. These values differ slightly from the physical values $\left(\mathrm{R}_{\text {in }} \sim 3.5 \mathrm{~cm}\right.$ and $R_{\text {out }}=\sim 3.6 \mathrm{~cm}$ since energy can be shared between the two electrodes and also the patterning is not perfectly circular. There is also a difference between the germanium and silicon detectors because they were made at different times with slightly different mask designs. The inner region had an effective radius of $\mathrm{R}_{\mathrm{in}}=3.58 \mathrm{~cm}$ for germanium and $\mathrm{R}_{\mathrm{in}}=3.54 \mathrm{~cm}$ for silicon and the outer region annulus spanned from $\mathrm{R}=3.72 \mathrm{~cm}(\mathrm{Ge})$ and $\mathrm{R}=3.64 \mathrm{~cm}(\mathrm{Si})$ out to $\mathrm{R}_{\max }=3.8 \mathrm{~cm}$. In the "shared" region between electrodes, the charge energy is shared with a simple linear model in this region such that $E_{q o}=\frac{R-R_{i}}{R_{o}-R_{i}} E_{s}$ and $E_{q i}=E_{s}-E_{q o}$.

This Monte Carlo simulation starts with an isotropic spectrum of neutrons from a $220 \mathrm{~cm}$ cube surrounding the outer shield of the experiment with a flux of $(2.14 \pm$ $0.02) \times 10^{-6}$ neutrons $/ \mathrm{cm}^{2} / \mathrm{sec}$ [4. This flux was based on an earlier simulation of fast neutrons at SUF using neutron production rates taken from the literature, which are uncertain by a factor of four [4. The absolute flux is relatively unimportant as all of the subsequent calculations are based on ratios of quantities in the Monte Carlo. For 
each event, which is a collection of hits following the results of an initial neutron and its subsequent daughter particles, we can use our electron recoil and nuclear recoil bands to infer the equivalent charge energy for each hit based on the particle type in the simulation. We add noise to the recoil, q-inner, and q-outer energies separately according to the characterizations which we made previously and then compute the efficiency as a function of energy based on these simulated events.

Since WIMPs do not multiple scatter - internally or otherwise - we can simply compute the efficiency of the cuts given a particular recoil energy. The efficiency for WIMPs shown in Figure 8.2 is a slight overestimate to the efficiency since a bug in the code did not properly add noise to the simulated energy. This efficiency should not be too much of an overestimate since the WIMP efficiency should be larger than the neutron efficiency due to the lack of internal multiple-scatters.

Figure 8.2 shows the efficiency of our cuts as a function of energy normalized to the maximum. This requires an adjustment to the livetime, such that the exposure of our $3 \times 250 \mathrm{~g}$ germanium detectors for neutrons after cuts is $50.9 \mathrm{~kg}$ days and the exposure for WIMPs is $54.3 \mathrm{~kg}$ days.

We can also compare the cut efficiency of the new cut with the efficiency of the flat cuts as in Figure 8.3. The two curves differ only in the "beta-eliminating" cut. Both cuts have approximately the same average efficiency when convolved with the expected energy spectrum of the neutron background $(\sim 29 \%)$, but have significantly different shapes.

\subsection{WIMP Candidates}

The single-scatter hits in germanium detectors provide us with our WIMP candidates. After all of the cuts (data quality cuts, fiducial volume cut, veto-anticoincident cut) except for the beta-eliminating cut, 263 low-yield, single-scatter events remain in detectors Z2, Z3, and Z5. Using the $2 \sigma$ nuclear recoil band cut with a flat risetime cut as in the previous analysis yields 29 WIMP candidates. Using the new betaeliminating cut yields 19 WIMP candidates. The low-yield events are plotted in Figure 8.4 with the 19 WIMP candidates circled.

\subsection{Measuring the Neutron Background}

Multiple-scatter events are a good measure of the neutron background since the probability of a WIMP multiple scatter is negligible. As discussed in the previous chapter, we have seen a clearly identifiable population of neutrons in the triple scatters and non-nearest-neighbor multiple scatters, but not in double scatters between adjacent 


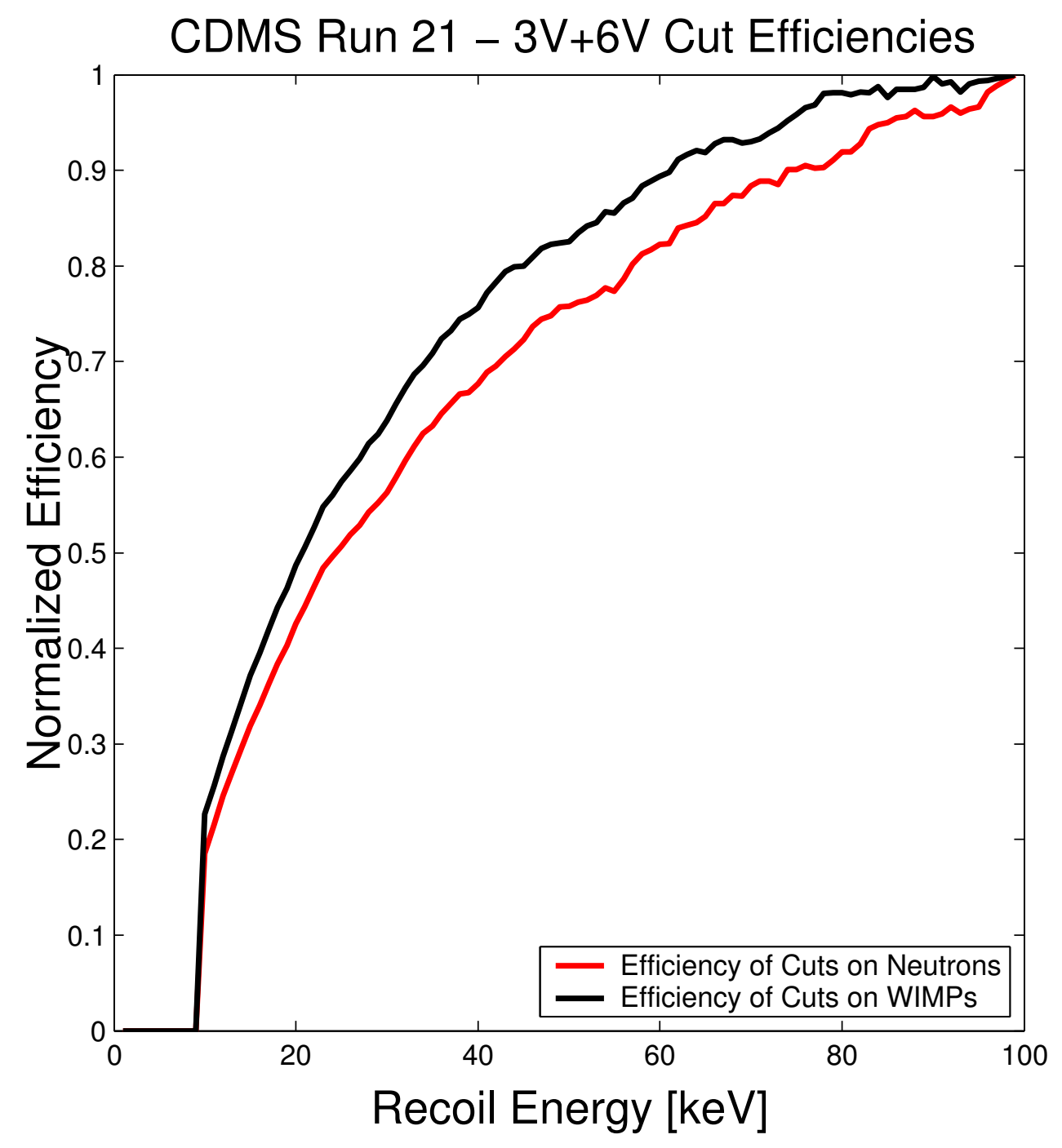

Figure 8.2: Combined Efficiency for Cuts in the Low-Background Germanium SingleScatters. The red curve is based on a neutron Monte Carlo and the black curve is based on the same Monte Carlo with both external and internal multiple scatters removed, which models a WIMP distribution. The efficiencies have been normalized such that the maximum value is at $100 \%$. 

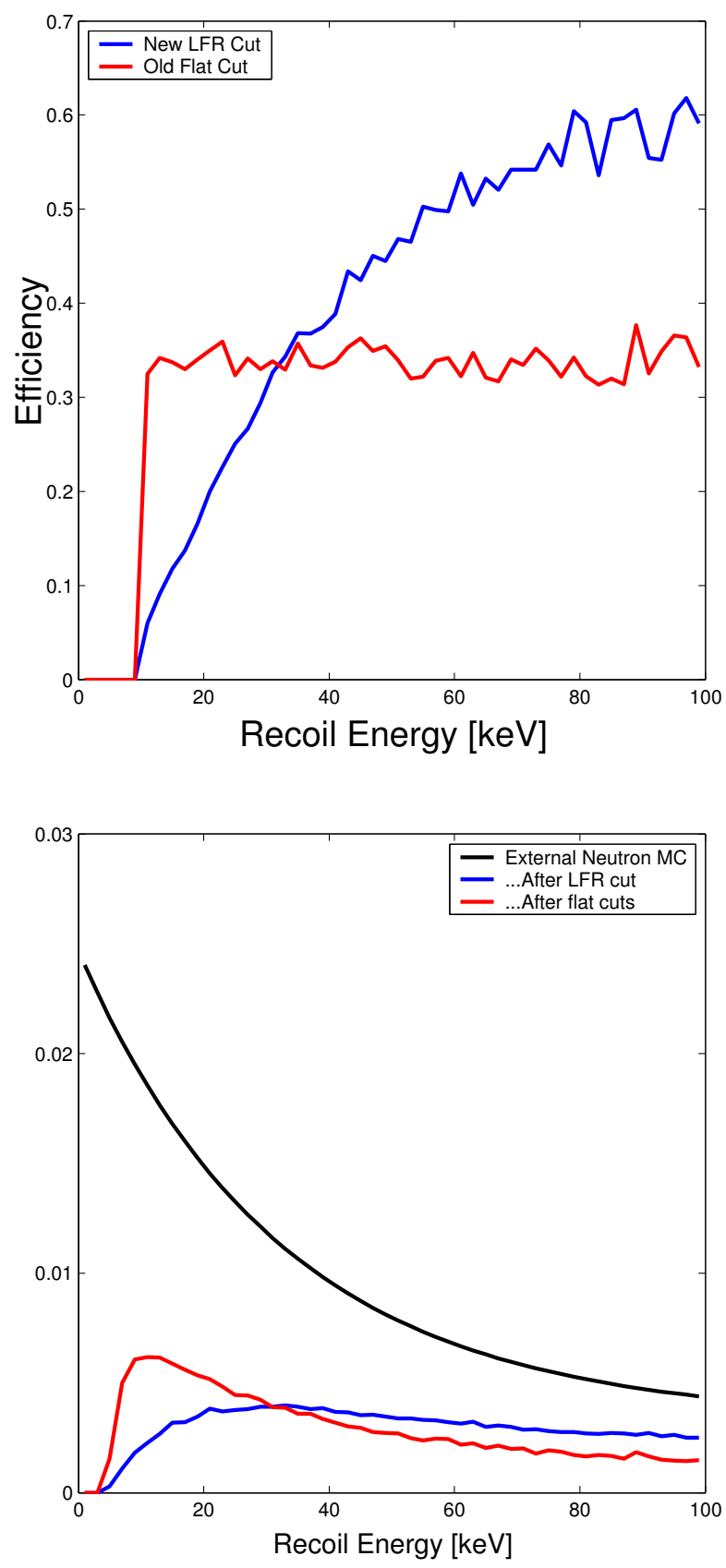

Figure 8.3: Comparison of Efficiencies Between New (LFR) and Old (Flat) Cuts. The two curves use the same combination of cuts except for the "beta-eliminating" cut. The blue curve uses the new maximum likelihood-based (LFR) method, while the red curve uses a flat risetime cut combined with a cut on the nuclear recoil band. The top graph shows the raw efficiencies as a function of recoil energy, while the bottom graph shows the cuts convolved with the expected neutron background spectrum. The two cuts have approximately the same total efficiency averaged over energy, but a much different shape as a function of energy. 


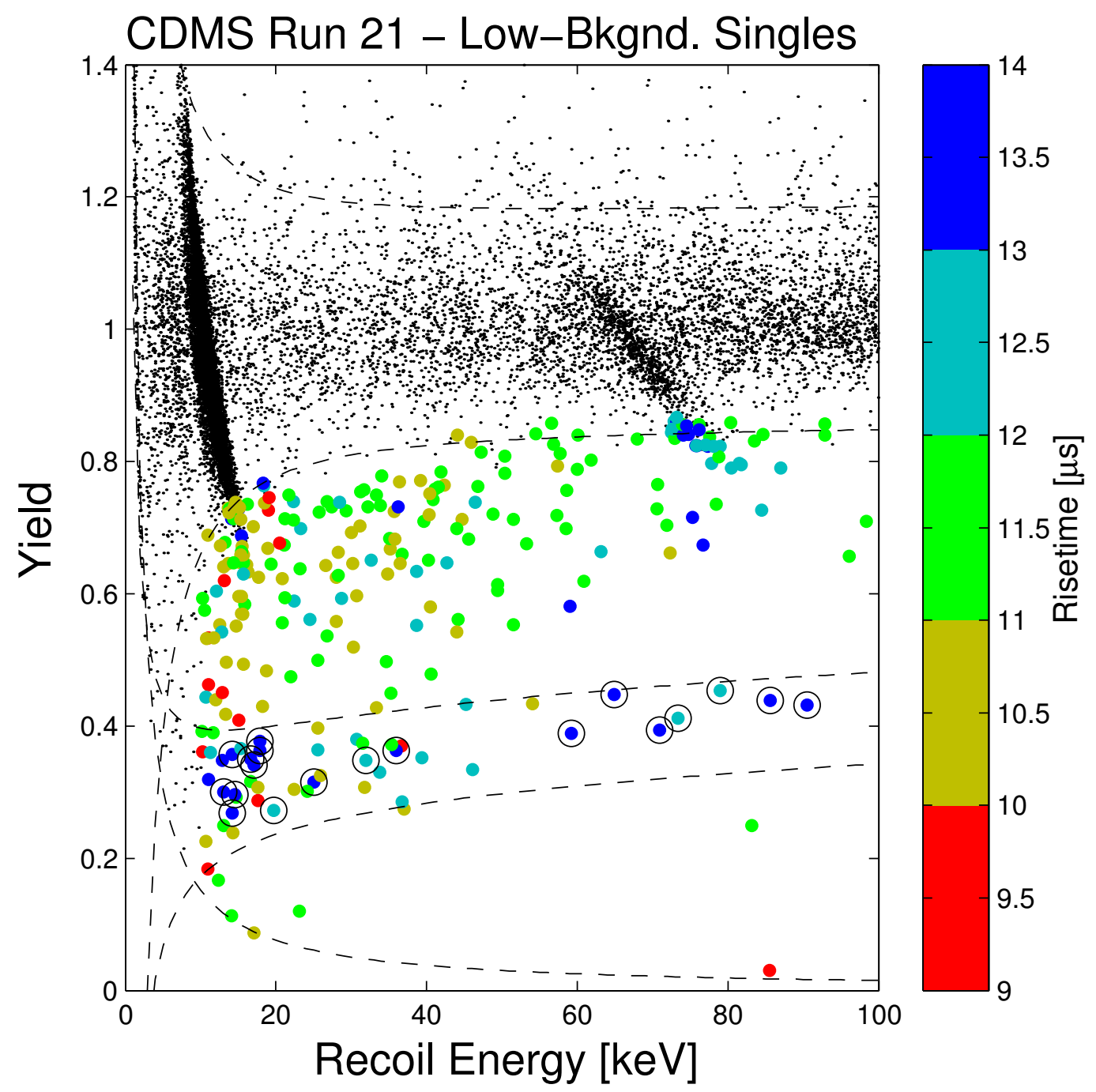

Figure 8.4: Distribution of Veto-Anticoincident, Low-Background, Single-Scatter Events in Z2, Z3, and Z5 in the Yield-vs.-Energy Plane. The two dashed lines around a yield of one represent the electron-recoil band and the two dashed line around a yield of 0.3 represent the nuclear-recoil band. The hyperbolic dashed line represents the charge threshold. Events in the electron-recoil band (small, black points) are excluded. Events in the low-yield region (shown as large circles) are color-coded by their risetime. Events which pass the LFR beta-eliminating cut, and are therefore identified as nuclear recoils (and hence WIMP candidates), are circled. Note that the cut keeps all of the events in the nuclear recoil band at higher energies, as would be expected from the distributions in Figure 7.9. Also note that the events that pass the cut at lower energies are those at high risetimes, as would be expected from the distributions in Figure 7.10. 


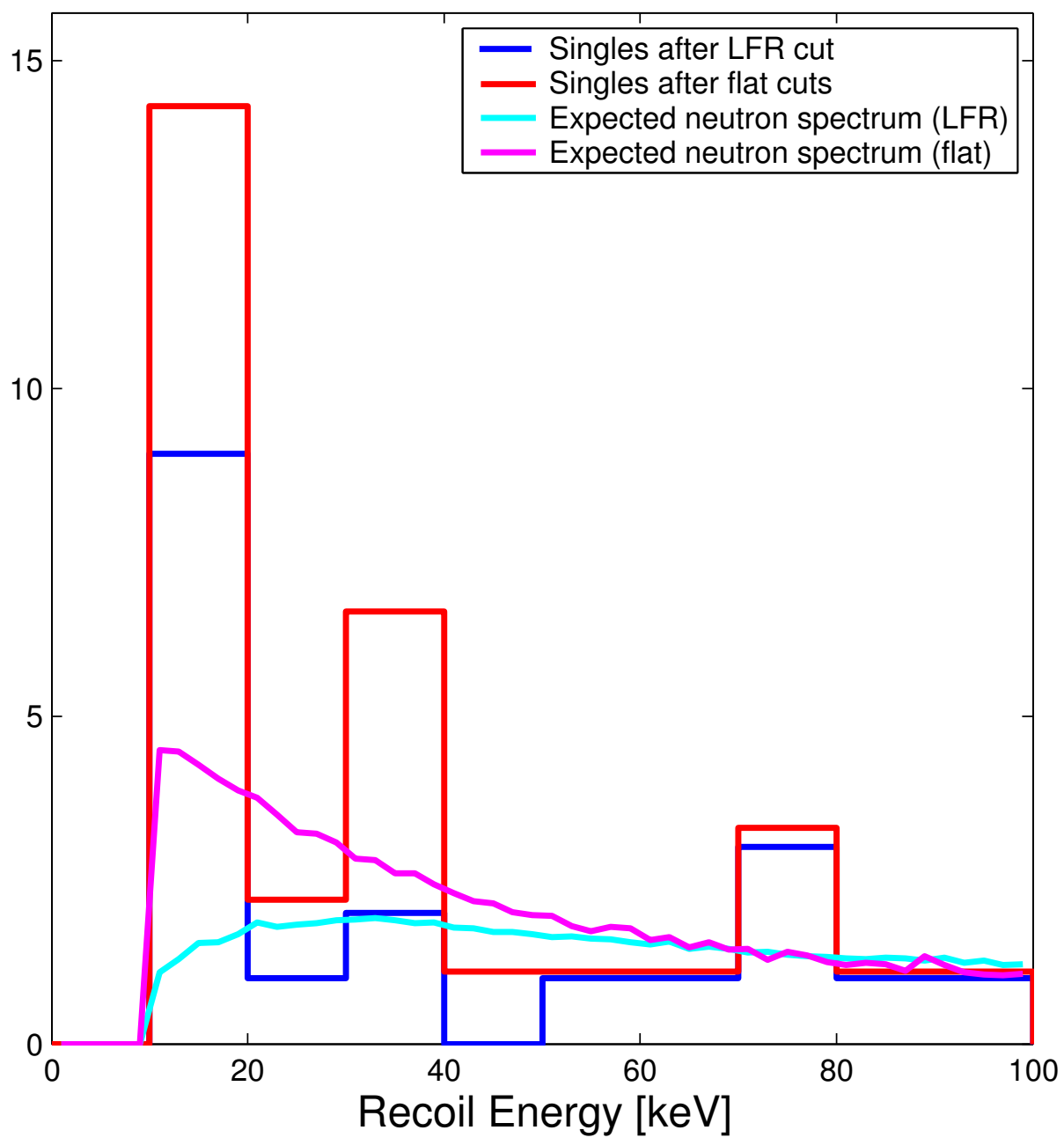

Figure 8.5: Comparison of WIMP Candidates using the Old and New Cuts. The blue (LFR) and red (Flat) histogram show the events which remain after the cuts and the cyan (LFR) and magenta (Flat) curves represent the expected spectrum based on the external neutron Monte Carlo. Both of these curves have been normalized to match the number of events with recoil energies greater than $50 \mathrm{keV}$ (there are 7 using either set of cuts). These events should not be betas since they are above the range where most of the leakage into the nuclear recoil band occurs (as discussed in Figure 8.4). 
detectors. These nearest-neighbor doubles had a significant contamination by events leaking down from the beta-beta events. We can use our beta-rejection cut to reduce this contamination to negligible levels. The cut levels must be very severe since any beta contamination that remains would lead to an overestimate of our neutron background.

Single-scatter events in the silicon detectors could also be used as a measure of the neutron background because silicon has a higher cross-section for neutrons than germanium as well as a lower cross-section for WIMPs. We do not use the silicon singles, however, since we do not have a sufficient characterization of the betas to eliminate all of them from the NNDs using our analysis while keeping an appreciable fraction of the neutrons (see Table 7.5).

Of the 126 NND events in the low-background set, 4 events pass our betaeliminating cut while only $\mathbf{1}$ event would pass the nuclear recoil band plus risetime cut. This ratio of $4 / 1$ is consistent with the estimate of $45 \% / 12.5 \%$ made in the previous chapter. A yield-vs.-yield plot of these events are shown in Figure 8.6.

There are $\mathbf{4 2 4}$ events in the low-background dataset which have low yield and are either a non-nearest neighbor double or a higher-order multiple scatter. Since the probability of a beta penetrating a detector is negligible, we do not need to make the complex beta-eliminating cut as in the single-scatters and the NND events. Using the usual nuclear recoil band and flat risetime cuts, 1 non-nearest neighbor double and 4 triple-scatters remain, which brings the total of multiple scatters to $\mathbf{9}$. The neutron Monte Carlo [3] predicts a ratio of single scatters to multiple scatters of 4.5 , which gives us $9 \times 4.5=40.5$ expected singles. A $\sqrt{N}$ (Poisson) statistical uncertainty on the measured number of multiple scatters (9) gives us an uncertainty of $\sqrt{9} \times 4.5=13.5$ in the expected number of neutron singles. This indicates that all of our 19 WIMP candidate germanium single-scatters are consistent with being neutrons. We are therefore background-limited and conservatively set an upper limit on the cross-section/mass parameter space allowed for WIMPs.

\subsection{Calculation of Cross-section/Mass Upper Limit}

Based on the spectrum of single-scatters and multiple-scatters (taken hit-per-hit) as shown in Figure 8.7 and the efficiencies to WIMPs and neutrons shown in Figure 8.2, we compute an upper limit in the cross-section/mass parameter space.

We can calculate a limit without statistical subtraction ("no subtraction" limit), that is, without making any assumptions about the neutron background. This limit is based on the "optimum interval" method developed by Steve Yellin [5], which effectively selects the interval of the energy spectrum which has the fewest observed events compared to the expected WIMP spectrum. This procedure is not appropriate 


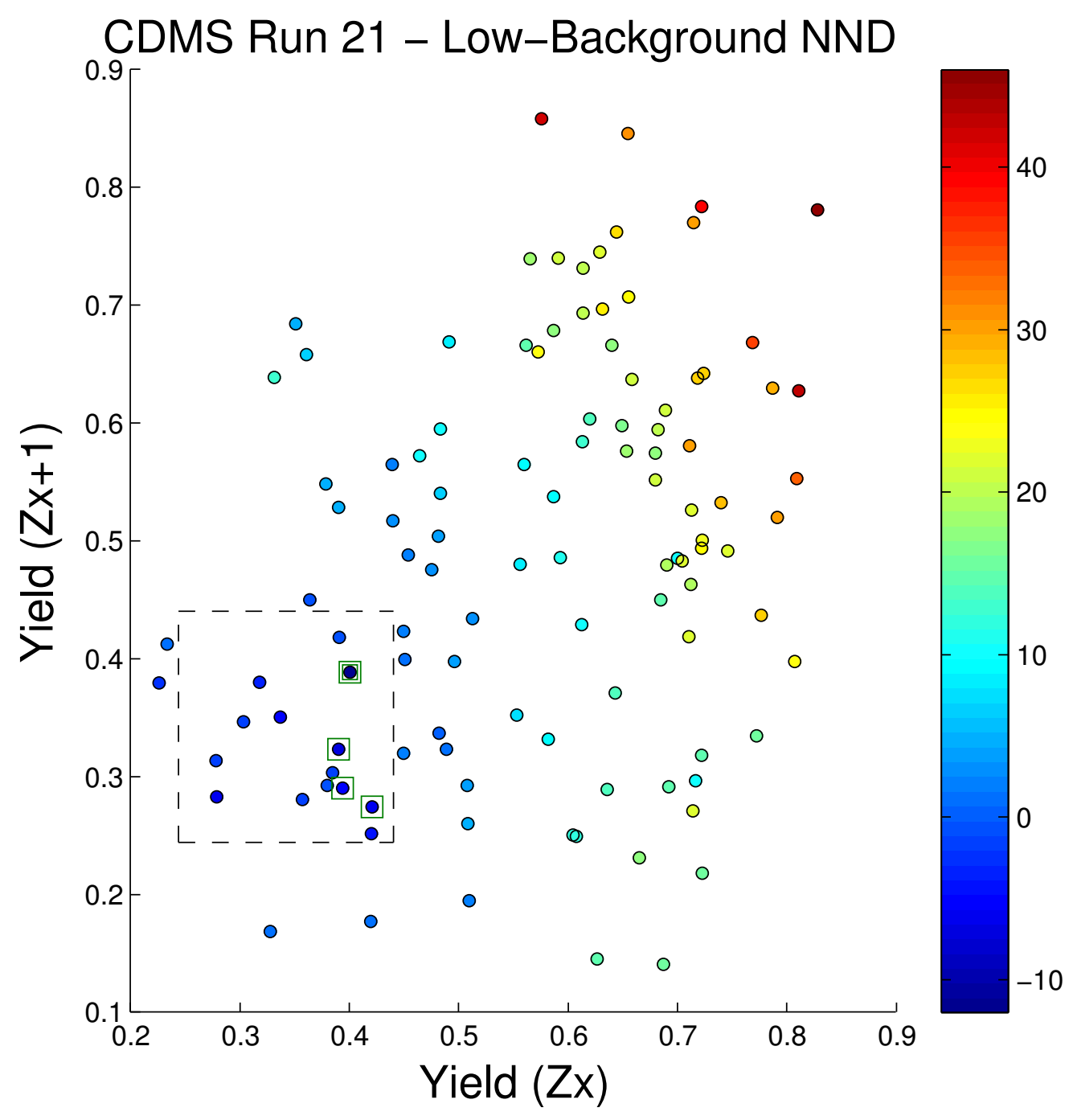

Figure 8.6: A Yield-vs.-Yield Plot for Nearest-Neighbor Double Scatters in the LowBackground Data. The color of the dot represents the value of $\mathcal{R}$. The dashed box represents an approximate nuclear recoil band and the selected events are highlighted by a green square (the heavier box represents an event which passes the flat cuts). Note the gradient in $\mathcal{R}$ from highest in the upper right to lowest inside the box (lower left). Based on the rough estimate of the efficiency for the nearest-neighbor cut developed in the previous chapter $(\sim 40 \%)$, we would expect that about 10 of the 15 events inside the box are neutrons. 

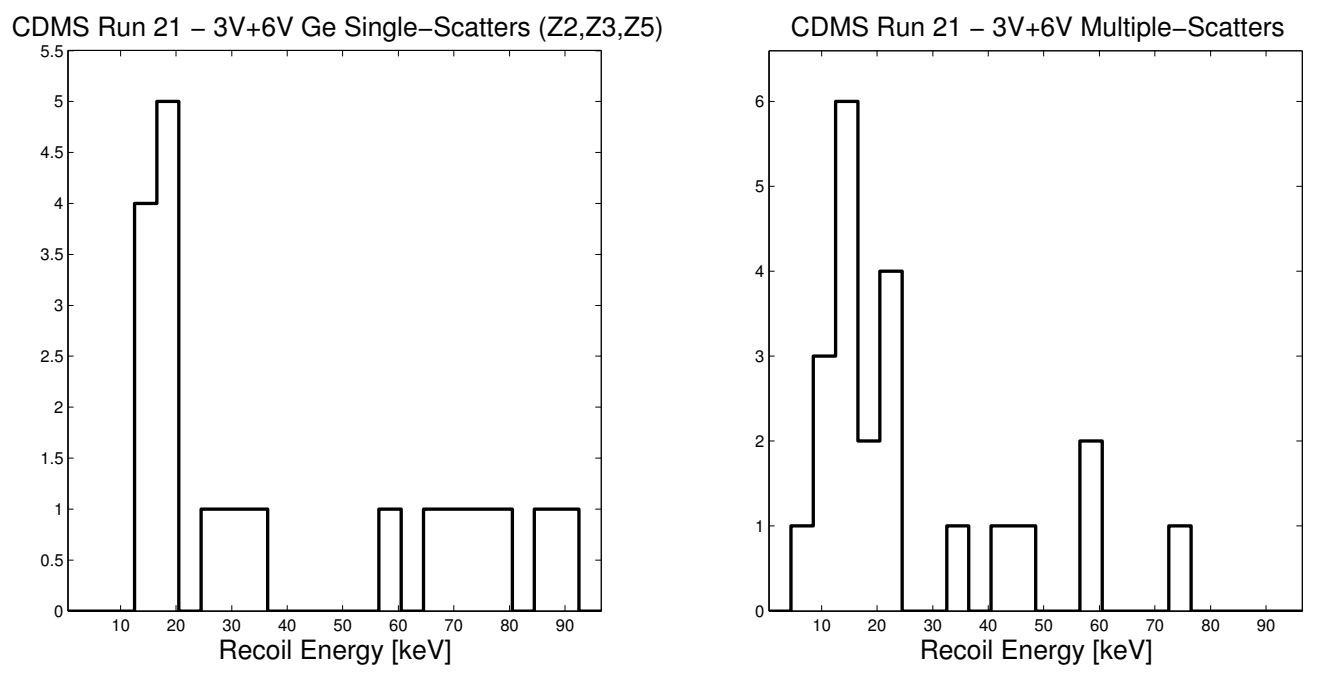

Figure 8.7: Energy Spectrum of Single- and Multiple-Scatters in the Low-Background Data. The energy spectrum of single-scatters in the germanium detectors Z2, Z3, and Z5 are shown at the left, while the energy spectrum of multiple-scatters in all detectors is shown on the right, where each hit has been included separately.

for the positive measurement of a signal, but rather assumes the presence of an unknown background and discards the portion of the spectrum where that background is the greatest. The method then produces a $90 \%$ confidence level upper limit based on the remaining portion of the spectrum with the appropriate statistical penalty for allowing this degree of freedom.

A second method uses an extension to the Feldman-Cousins method [6] [7] in order to use our measurement of the neutron background via the multiple-scatter events to make a statistical subtraction of that background. Based on the observed spectrum of germanium single scatters and all multiple-scatters (after cuts), we simulate a large number of possible outcomes to produce a distribution of the ratio:

$$
R(\sigma, M)=\frac{L\left(E_{i}, N_{m} \mid \sigma, M, \tilde{n}\right)}{L\left(E_{i}, N_{m} \mid \hat{\sigma}, \hat{M}, \hat{n}\right)}
$$

where $\hat{\sigma}, \hat{M}, \hat{n}$ are the physically allowed values of WIMP-nucleon cross-section, WIMP mass, and number of expected neutron events which maximize the likelihood $L$ of having an experiment with the simulated energy spectrum of single scatters $\left(E_{i}\right)$ and number of multiple scatters $\left(N_{m}\right)$. The value for $\tilde{n}$ is the maximally likely number of neutron events given $E_{i}$ and $N_{m}$ at the particular values of $\sigma$ and $M$. Based on this distribution, a critical value $R_{90}$ is found such that $90 \%$ of the simulations have 
a larger likelihood ratio. We then compute $R_{\text {data }}(\sigma, M)$ for the observed energy spectrum of single-scatters $\left(E_{i}\right)$ and the number of multiples $\left(N_{m}\right)$, where a point $(\sigma, M)$ is excluded from our parameter space if $R_{\text {data }}$ is less than $R_{90}$. We conservatively project out the value of $n$, the expected neutron background, by setting our $90 \%$ confidence level upper limit by only excluding values of $(\sigma, M)$ which are rejected for all possible values of $n$.

The limit for this analysis is shown in Figure 8.9. Also shown is the limit from the previous analysis of the $3 \mathrm{~V}$ data alone [8], based on 20 events in $28.3 \mathrm{~kg}$ days of data (after cuts). The new limit is worse at low masses due to a higher energy threshold (10 keV vs. $5 \mathrm{keV}$ ) combined with a lower efficiency at low energies (see Figure 8.3), but better at high masses due to the improved statistics on the measurement of the neutron background via the multiple-scatters. A future analysis will use two different levels for the beta-eliminating cut, one for calculating the limits for low-mass WIMPs and one for high-mass WIMPs as discussed in Section 7.5. This will improve our efficiency at low energies, which is the limiting factor for our low-mass WIMP limit.

\subsection{Comparison with Other WIMP Searches}

There are several other WIMP detection programs currently underway, some of which make a measurement of the thermal energy deposited by an interaction. Several novel approaches are used to increase the signal-to-noise ratio for a WIMP signal by rejecting background particles based on a simultaneous measurement of another quantity of the interaction in a manner similar to CDMS. A comparison plot of some of these experiments is shown in Figure 8.9. The solid lines represent $90 \%$ confidence level upper limits on the cross-section for a given WIMP mass. Also included is the $3 \sigma$ allowed region claimed by the DAMA experiment (see below) and an area representing a region allowed by some formulations of the Minimal Supersymmetric Standard Model (MSSM) [9].

The DAMA experiment (DArk MAtter search [12]) uses $100 \mathrm{~kg}$ of NaI scintillators located in the Gran Sasso National Laboratory in Italy. Their main backgroundrejection tool is to look for a modulation of the flux of WIMP candidate events due to the the varying velocity of the earth through the galactic halo as the earth revolves around the sun. Although they use a significantly different detector technology and detection methodology than CDMS, the DAMA experiment merits mention here since they claim to have made a detection of this annual modulation based on about 60,000 kg-days of exposure. Their analysis favors a WIMP mass of $M_{W}=44 \mathrm{GeV} / \mathrm{c}^{2}$ and cross-section of $\sigma=5.4 \times 10^{-42} \mathrm{~cm}^{2}$ [13] for a spin-independent (SI) interaction. Since the sodium and the iodine nuclei used as targets in this detector both have non-zero spin, DAMA is also sensitive to a spin-dependent (SD) interaction. This allows for a 


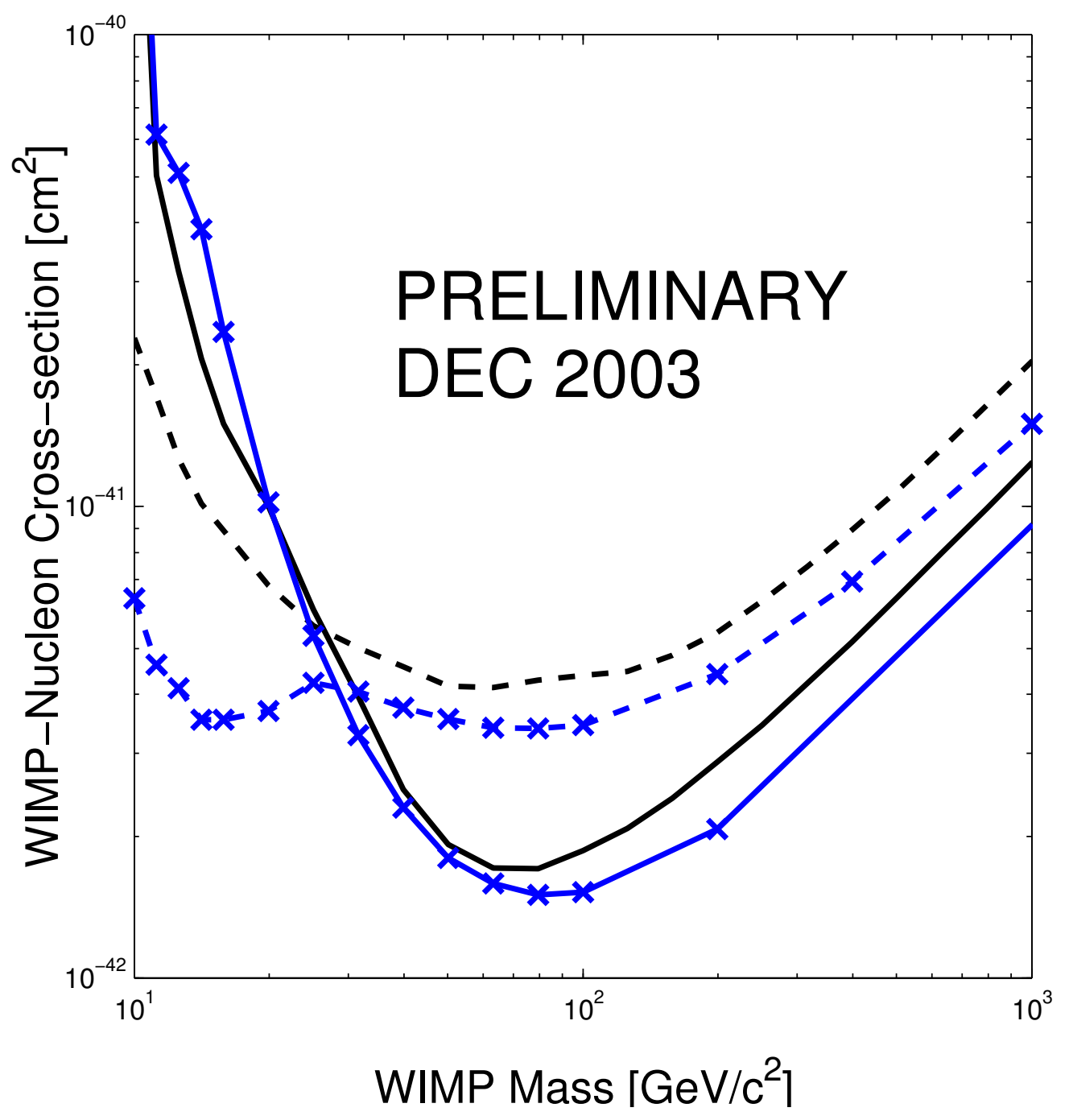

Figure 8.8: Spin-Independent WIMP-Nucleon Cross-section vs. WIMP Mass 90\% Confidence Level Upper Limits. The previous (3V only [8]) CDMS limits are shown as dashed lines while the new limits from this analysis are shown as solid lines. The black curves represent the "no-subtraction" limits, that is, a limit found using Steve Yellin's Optimum Interval method [5] which assumes no knowledge of the neutron background. The blue curves with "X" markers represent the limits using a FeldmanCousins subtraction of a background of neutrons based on Monte Carlo simulations as well as the observed background of multiple-scatter events. 


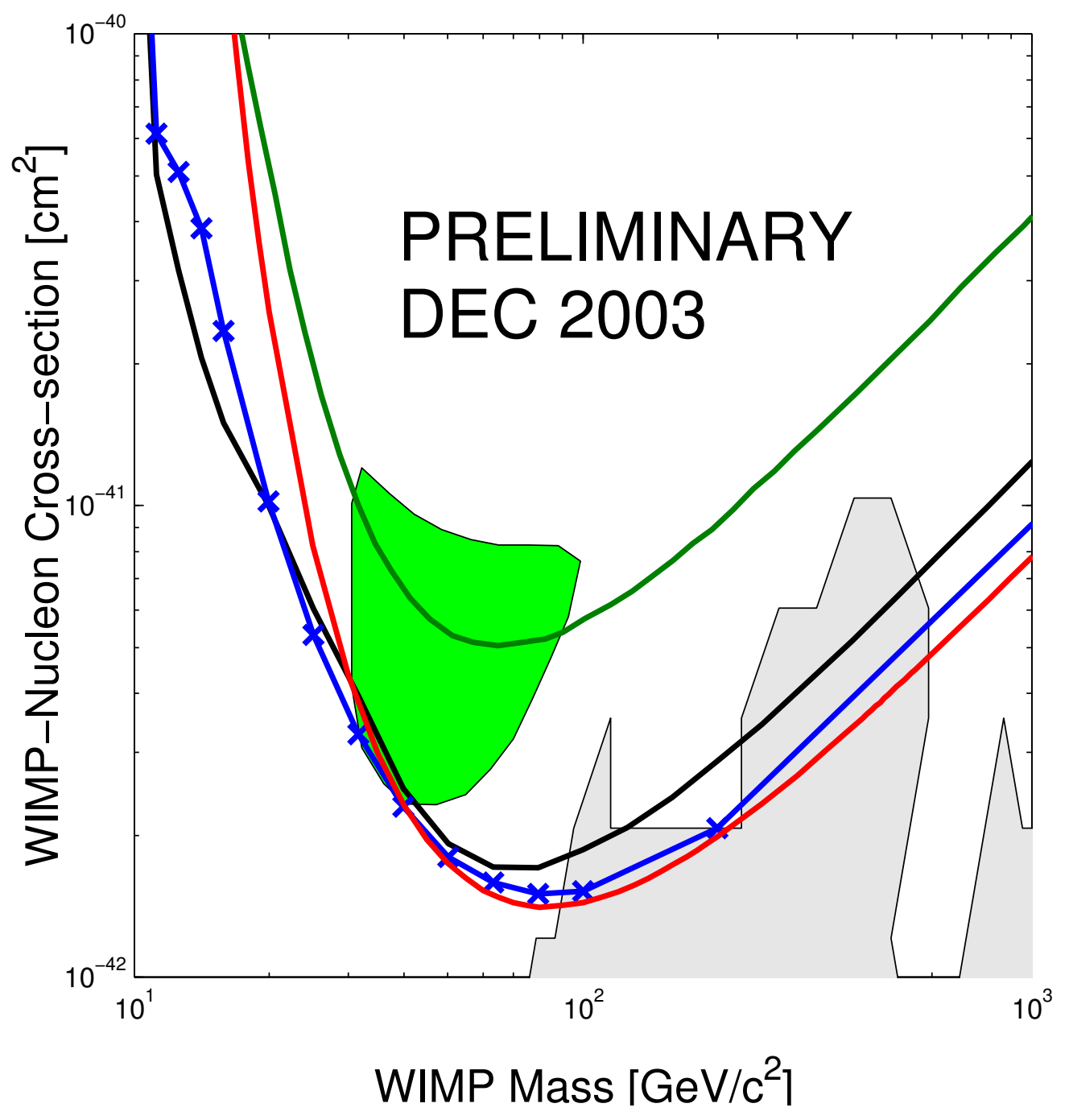

Figure 8.9: Spin-Independent WIMP-Nucleon Cross-section vs. WIMP Mass 90\% Confidence Level Upper Limits. The new "no-subtraction" (optimal interval) CDMS limit is shown as a solid black curve and the background-subtracted (FeldmanCousins) limit is shown as a blue curve. Also included as a comparison are the DAMA 1996 upper limit [10] (dark green curve), the claimed DAMA $20003 \sigma$ detection region (green filled region), and the EDELWEISS 2002 upper limit [11] (red curve). The grey shaded region represents a region of parameter space allowed by some formulations of MSSM [9]. 
larger region of allowed values for mass and cross-section which is some function of the two types of interactions, $\sigma=F\left(\sigma_{S I}, \sigma_{S D}\right)$. There are two plots of DAMA results in Figure 8.9. The filled area shows the $3 \sigma$ detection region reported in [13] and the line represents a $90 \%$ confidence level upper limit reported in [10].

The EDELWEISS experiment (Expérience pour DEtecter Les WIMPs En Site Souterrain [14]) uses 3-320g, cryogenically-cooled germanium detectors. They measure the heat signal of the interaction by measuring the change in temperature of the detector with Neutron-Transmutation-Doped (NTD) thermistors. Like CDMS, the EDELWEISS detectors make a simultaneous measurement of the ionization signal of the interaction. The current EDELWEISS limits are based on 8.6 kilogram-days of data in which no nuclear recoil events were seen in the range of 20-200 keV.

\subsection{Outlook}

The CDMS limits shown in Figure 8.8 are currently limited by our neutron background. This background is dominated by muon-induced neutrons since we have been running at a shallow site ( $\sim 10$ meters depth). Future results will be based on data taken at our deep site in the Soudan Underground Laboratory in Minnesota, which should provide about two orders of magnitude improvement in our sensitivity based on the reduction of this background by the $\sim 700$ meter overburden there.

At this depth, we expect that the limiting background will most likely be betas rather than neutrons. In order to potentially confirm a WIMP signal under these conditions, we must have an understanding of this background. The beta-elimination method developed in the previous chapter will be critical in characterizing such a background. Specifically, we will need to be able to use the spectrum of betas outside our signal region combined with the shape of the beta distribution in the risetimeyield plane to estimate the spectrum of events which leak into our signal region. In order to make this estimate with confidence, we must have a better understanding of the distribution of betas. There are three efforts currently underway in this respect. First, we have data taken with one of our next batch of detectors (G31 from "Tower 2") which was exposed to a collimated beta calibration source. There is a lot still to be understood with this data, but it should provide valuable insight into the physics of surface events in our detectors. Second, our test facility here at CWRU has upgraded its equipment to allow for the simultaneous readout of two detectors. This will allow us to study NND (nearest-neighbor double) events like those we have used in defining our beta likelihood function in Chapter 7. The subset of these events that we are interested in have recently been dubbed "ejectrons" since they occur when a gamma interacts near the surface and ejects and electron which travels to the neighboring detector. Finally, we are also exploring the possibility of performing 
a separate ejectron calibration run at Soudan by tailoring the trigger for nearestneighbor double scatters, which should provide a larger statistical sample to base our fits on compared to using serendipitous events in the gamma calibration. The combination of a better understanding of the shape of the beta distribution with an increased sample for performing the fits will allow us to perform a statistical subtraction of a beta background with the same confidence that we now do for a neutron background. 


\section{Works Cited}

[1] Angela Reisetter. A new veto cut using charge times and reprocessed data. CDMS Run 21 ebook \#296, June 2003.

[2] Sharmila Kamat. Estimating the limiting neutron background seen in run 21. CDMS Internal Note, January 2003.

[3] Sharmila Kamat. Comparison of the external neutron Monte Carlo simulations to the $6 \mathrm{~V}$ data. CDMS Internal Note, June 2003.

[4] T.A. Perera. The Limiting Background in a Dark Matter Search at Shallow Depth. PhD thesis, Case Western Reserve University, January 2002.

[5] S. Yellin. Physical Review D, 57:3873, 1998.

[6] G.J. Feldman and R. Cousins. Phys. Rev. D, (57):3873, 1998.

[7] Richard Schnee. Memo on calculating CDMS limits following Feldman and Cousins. CDMSnote 0105006, May 2001.

[8] T. Saab. Search for Weakly Interacting Massive Particles with the Cryogenic Dark Matter Search Experiment. PhD thesis, Stanford University, August 2002.

[9] E.A. Baltz and P. Gondolo. Implications of muon anomalous magnetic moment for supersymmetric dark matter. 2001. hep-ph/0102147.

[10] R. Bernabei et al. New limits on WIMP search with large-mass low-radioactivity Na-I(Tl) set-up at gran sasso. Physical Review B, 389:757-766, December 1996.

[11] A. Benoit et al. Improved exclusion limits from the EDELWEISS WIMP search. June 2002. astro-ph/0206271.

[12] P. Belli et al. DAMA Dark Matter Search. 1998. http://www.lngs.infn.it/lngs/report98/dama.pdf.

[13] R. Bernabei et al. Search for WIMP annual modulation signature: results from DAMA/NaI-3 and DAMA/NaI-4 and the global combined analysis. Physical Review B, 480:23-31, March 2000.

[14] V. Sanglard et al. The EDELWEISS experiment and dark matter direct detection. June 2003. astro-ph/0306233. 


\section{Appendix A}

\section{Calibration Data}

\section{A.1 Search Thresholds}

Figures A.1, A.2, A.3, and A.4 contain detector-by-detector plots of the search thresholds as described in Section 6.2. The levels are based on a Gaussian fit to randomlytriggered data.

\begin{tabular}{|r|r|r|r|r|r|}
\hline 3V & $2 \sigma$ & $3 \sigma$ & $4 \sigma$ & $5 \sigma$ & $6 \sigma$ \\
\hline Z1 & 34700.8544 & 2059.0041 & 48.3083 & 0.4372 & 0.0015 \\
\hline Z2 & 32536.7565 & 1930.5956 & 45.2955 & 0.4100 & 0.0014 \\
\hline Z3 & 40329.5083 & 2392.9850 & 56.1441 & 0.5082 & 0.0017 \\
\hline Z4 & 34875.1060 & 2069.3435 & 48.5508 & 0.4394 & 0.0015 \\
\hline Z5 & 37692.6869 & 2236.5270 & 52.4733 & 0.4749 & 0.0016 \\
\hline Z6 & 55223.5267 & 3276.7340 & 76.8786 & 0.6958 & 0.0024 \\
\hline
\end{tabular}

Table A.1: Estimated number of noise events misidentified as a signal given various search threshold levels (3V). The values quoted are for 100 livedays, the approximate livetime of the experiment. 
CDMS Run 21 - 3V Low-Background Data - Z1

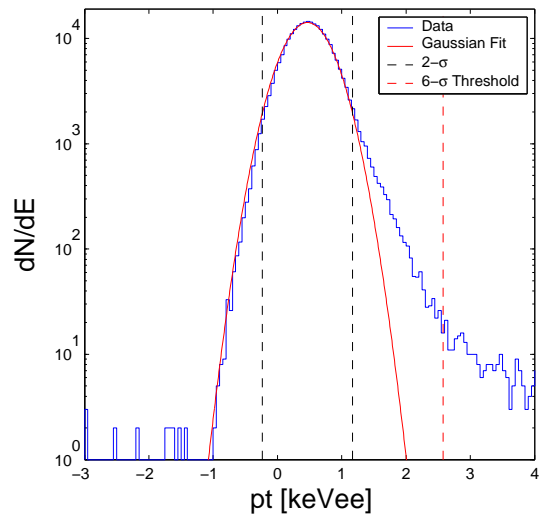

CDMS Run 21 - 3V Low-Background Data - Z3

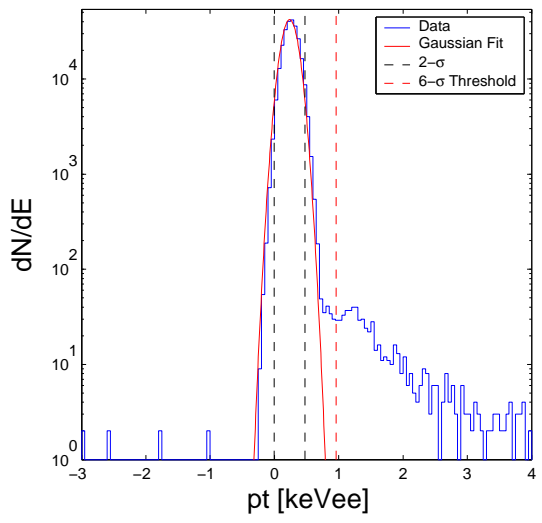

CDMS Run 21 - 3V Low-Background Data - Z5

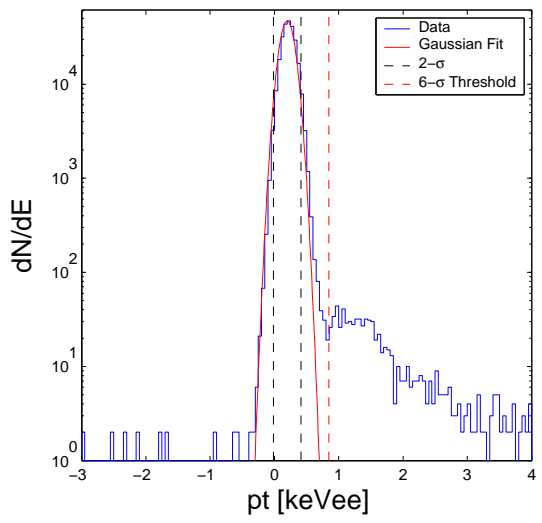

CDMS Run 21 - 3V Low-Background Data - Z2

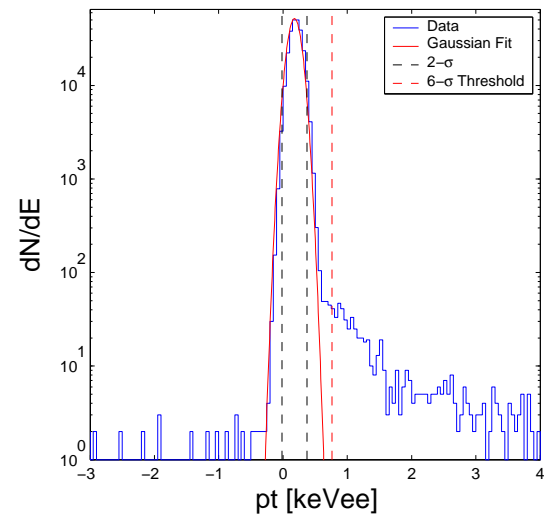

CDMS Run 21 - 3V Low-Background Data - Z4

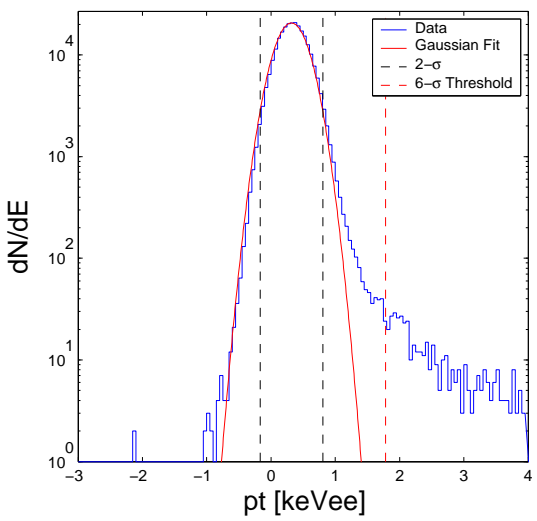

CDMS Run 21 - 3V Low-Background Data - Z6

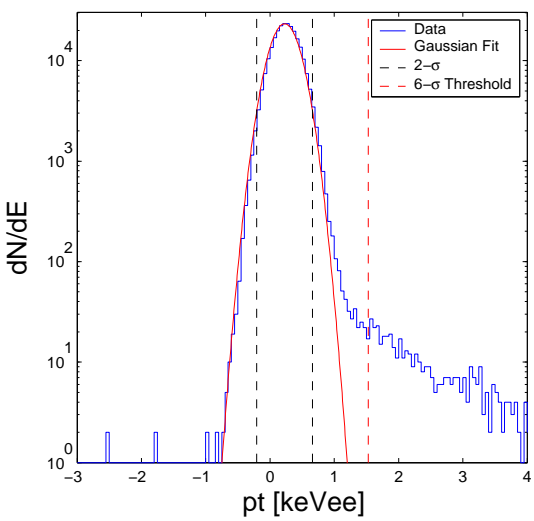

Figure A.1: Gaussian Fit of Phonon Noise Levels Based on Randomly-Triggered Data $(3 \mathrm{~V})$. The blue line is the data and the red line is the Gaussian fit. The black dashed lines represent the bounds of the $2 \sigma$ level of the fit and the red dashed line is the $6 \sigma$ cut level. 
CDMS Run 21 - 6V Low-Background Data - Z1

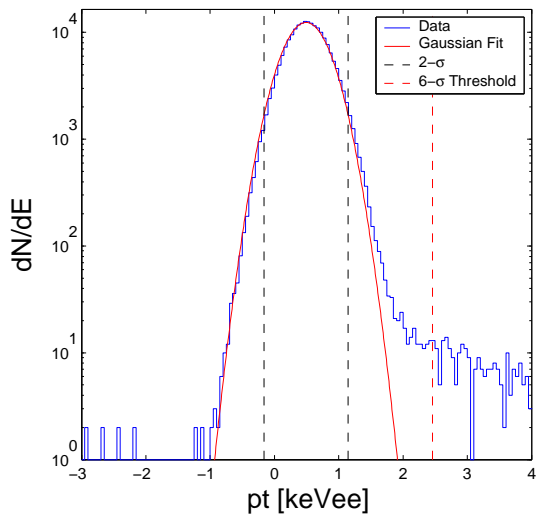

CDMS Run 21 - 6V Low-Background Data - Z3

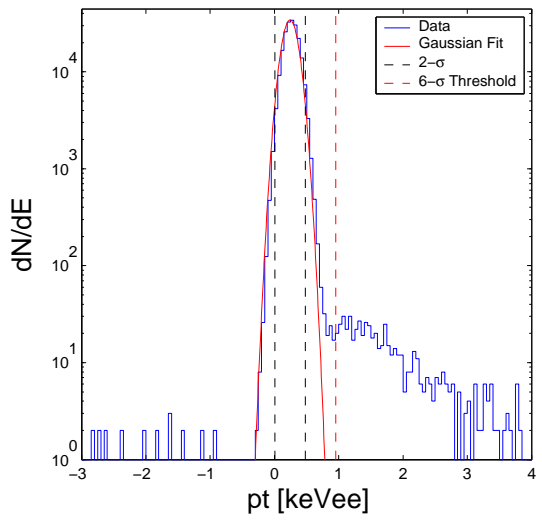

CDMS Run 21 - 6V Low-Background Data - Z5

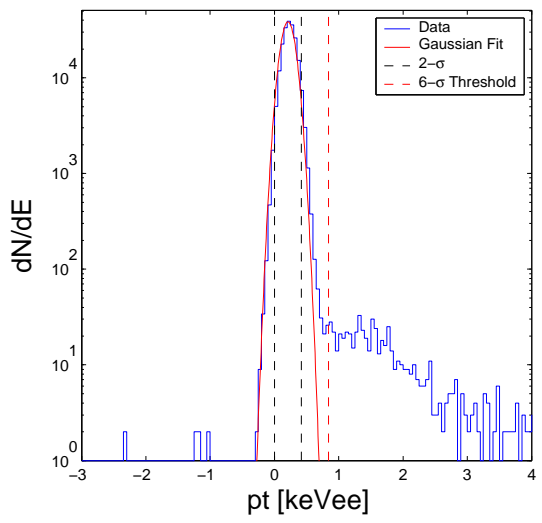

CDMS Run 21 - 6V Low-Background Data - Z2

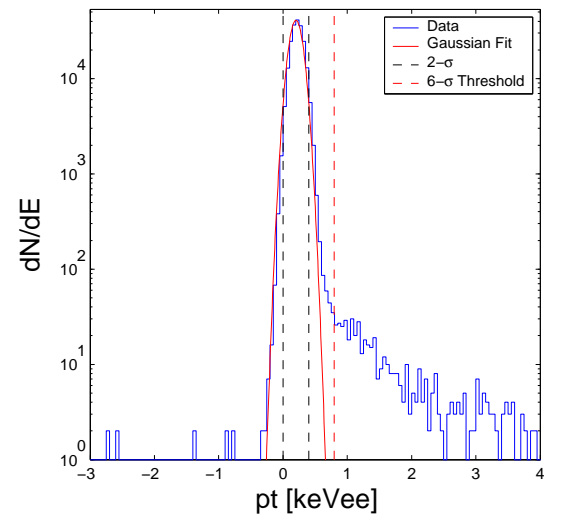

CDMS Run 21 - 6V Low-Background Data - Z4

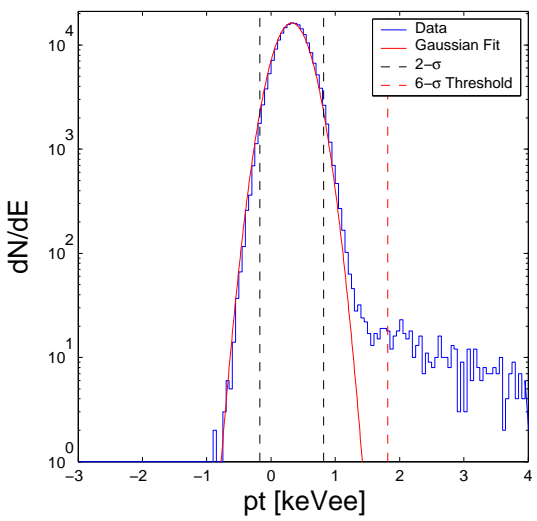

CDMS Run 21 - 6V Low-Background Data - Z6

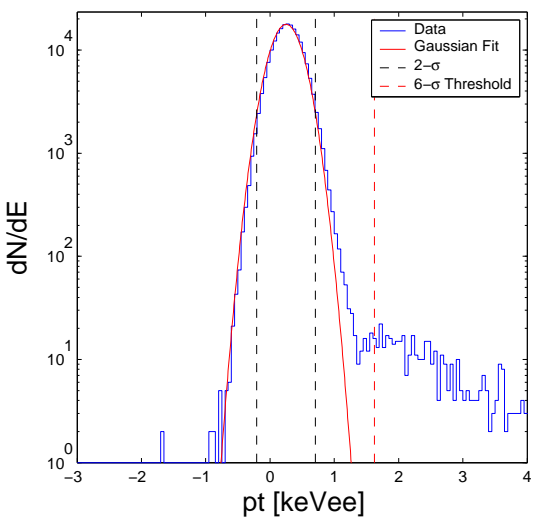

Figure A.2: Gaussian Fit of Phonon Noise Levels Based on Randomly-Triggered Data $(6 \mathrm{~V})$. The blue line is the data and the red line is the Gaussian fit. The black dashed lines represent the bounds of the $2 \sigma$ level of the fit and the red dashed line is the $6 \sigma$ cut level. 
CDMS Run 21 - 3V Low-Background Data - Z1

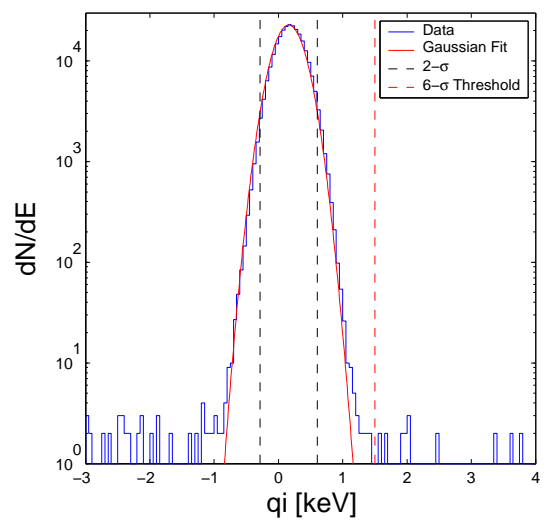

CDMS Run 21 - 3V Low-Background Data - Z3

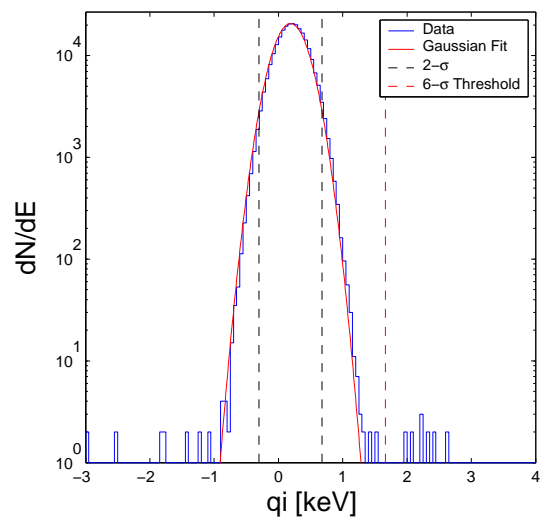

CDMS Run 21 - 3V Low-Background Data - Z5

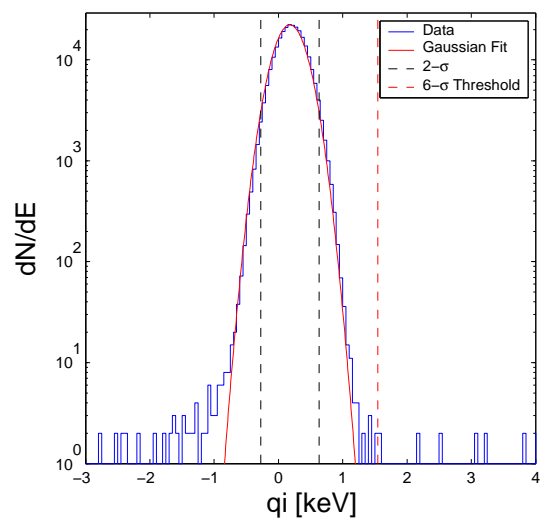

CDMS Run 21 - 3V Low-Background Data - Z2

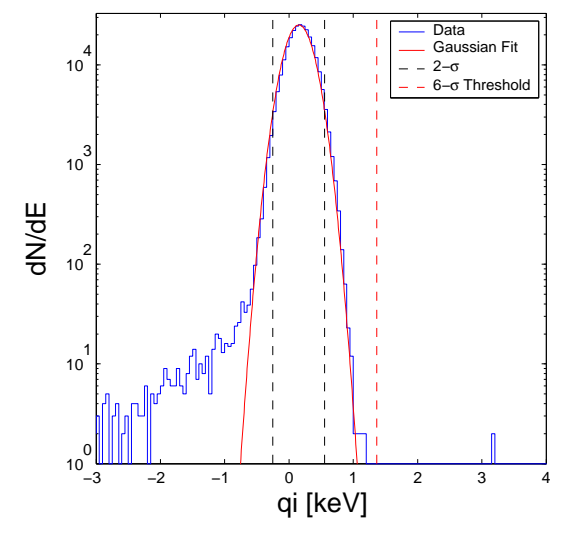

CDMS Run 21 - 3V Low-Background Data - Z4

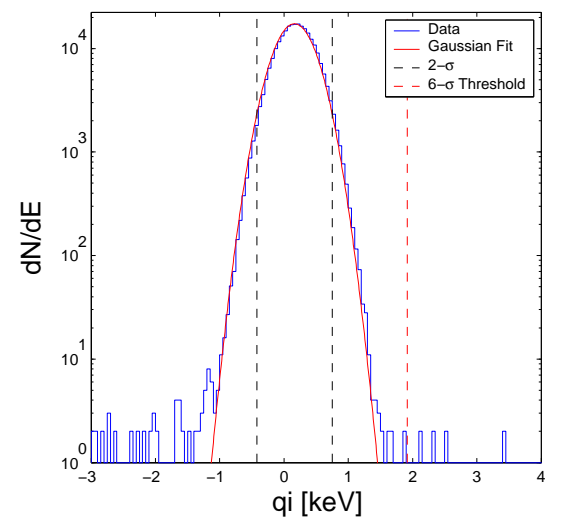

CDMS Run 21 - 3V Low-Background Data - Z6

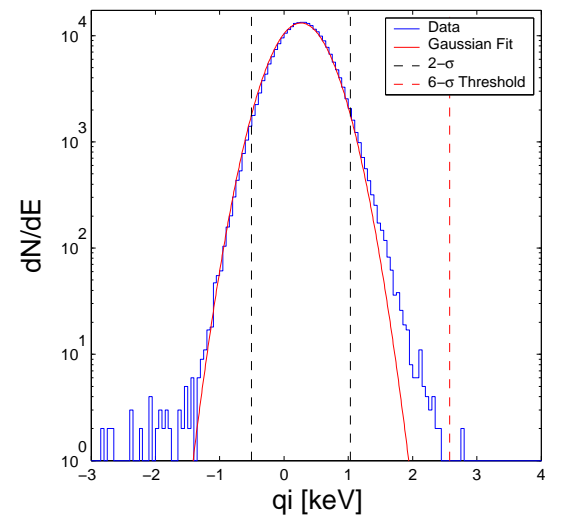

Figure A.3: Gaussian Fit of Charge Noise Levels Based on Randomly-Triggered Data $(3 \mathrm{~V})$. The blue line is the data and the red line is the Gaussian fit. The black dashed lines represent the bounds of the $2 \sigma$ level of the fit and the red dashed line is the $6 \sigma$ cut level. 
CDMS Run 21 - 6V Low-Background Data - Z1

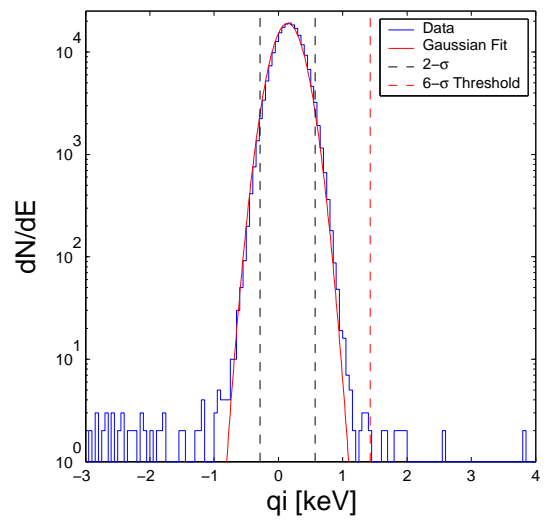

CDMS Run 21 - 6V Low-Background Data - Z3
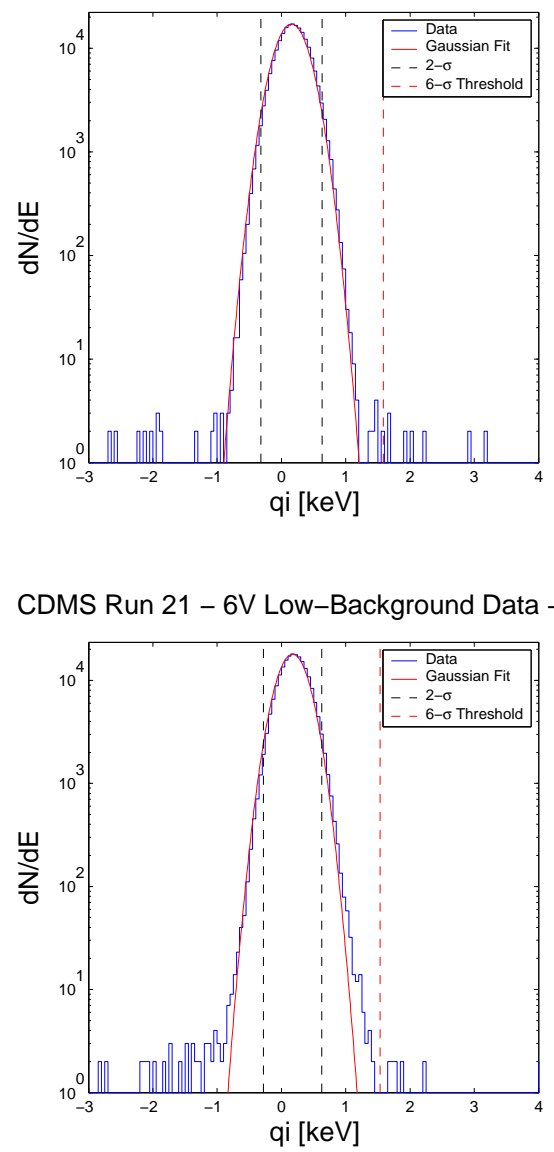

CDMS Run 21 - 6V Low-Background Data - Z2

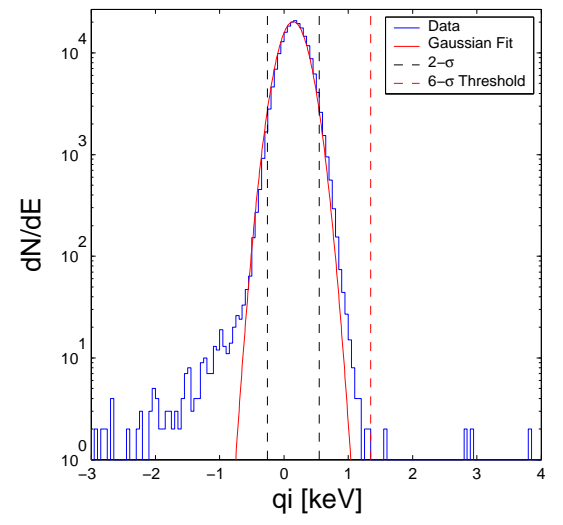

CDMS Run 21 - 6V Low-Background Data - Z4

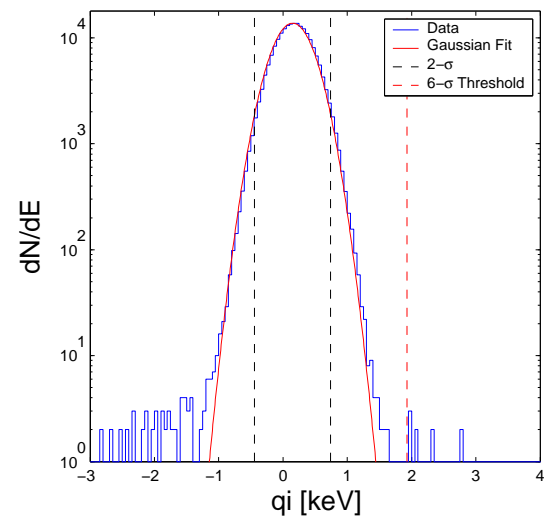

CDMS Run 21 - 6V Low-Background Data - Z6

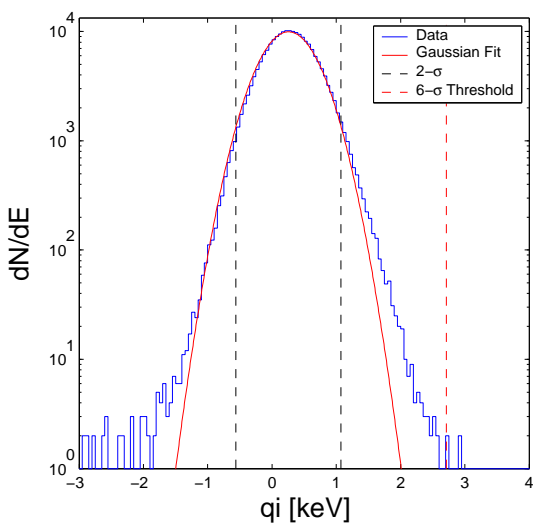

Figure A.4: Gaussian Fit of Charge Noise Levels Based on Randomly-Triggered Data $(3 \mathrm{~V})$. The blue line is the data and the red line is the Gaussian fit. The black dashed lines represent the bounds of the $2 \sigma$ level of the fit and the red dashed line is the $6 \sigma$ cut level. 


\begin{tabular}{|l|r|r|r|r|r|}
\hline 6V & $2 \sigma$ & $3 \sigma$ & $4 \sigma$ & $5 \sigma$ & $6 \sigma$ \\
\hline Z1 & 32669.5441 & 1938.4746 & 45.4804 & 0.4116 & 0.0014 \\
\hline Z2 & 31710.1236 & 1881.5466 & 44.1448 & 0.3995 & 0.0014 \\
\hline Z3 & 34756.4230 & 2062.3013 & 48.3856 & 0.4379 & 0.0015 \\
\hline Z4 & 33003.9061 & 1958.3143 & 45.9459 & 0.4158 & 0.0014 \\
\hline Z5 & 37935.1039 & 2250.9110 & 52.8108 & 0.4780 & 0.0016 \\
\hline Z6 & 54679.5850 & 3244.4587 & 76.1213 & 0.6890 & 0.0024 \\
\hline
\end{tabular}

Table A.2: Estimated number of noise events misidentified as a signal given various search threshold levels $(6 \mathrm{~V})$. The values quoted are for 100 livedays, the approximate livetime of the experiment. 


\section{A.2 Fiducial Volume Cut}

The following plots detail the definition of the q-inner noise thresholds and the q-outer noise bands described in Section 6.3. 

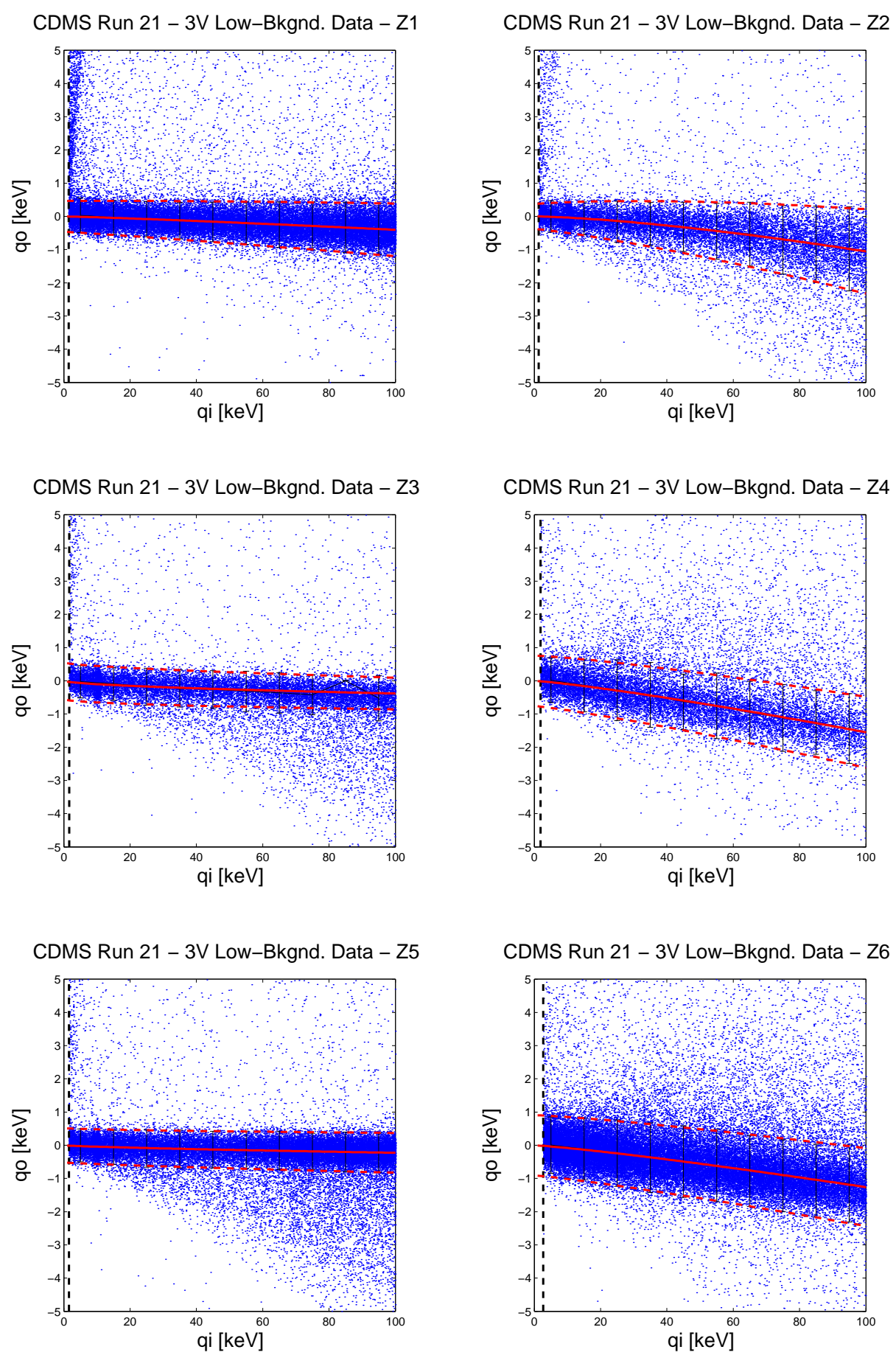

Figure A.5: Definition of the $6 \sigma$ q-inner noise threshold and the $2 \sigma$ q-outer noise bands $(3 \mathrm{~V})$. 

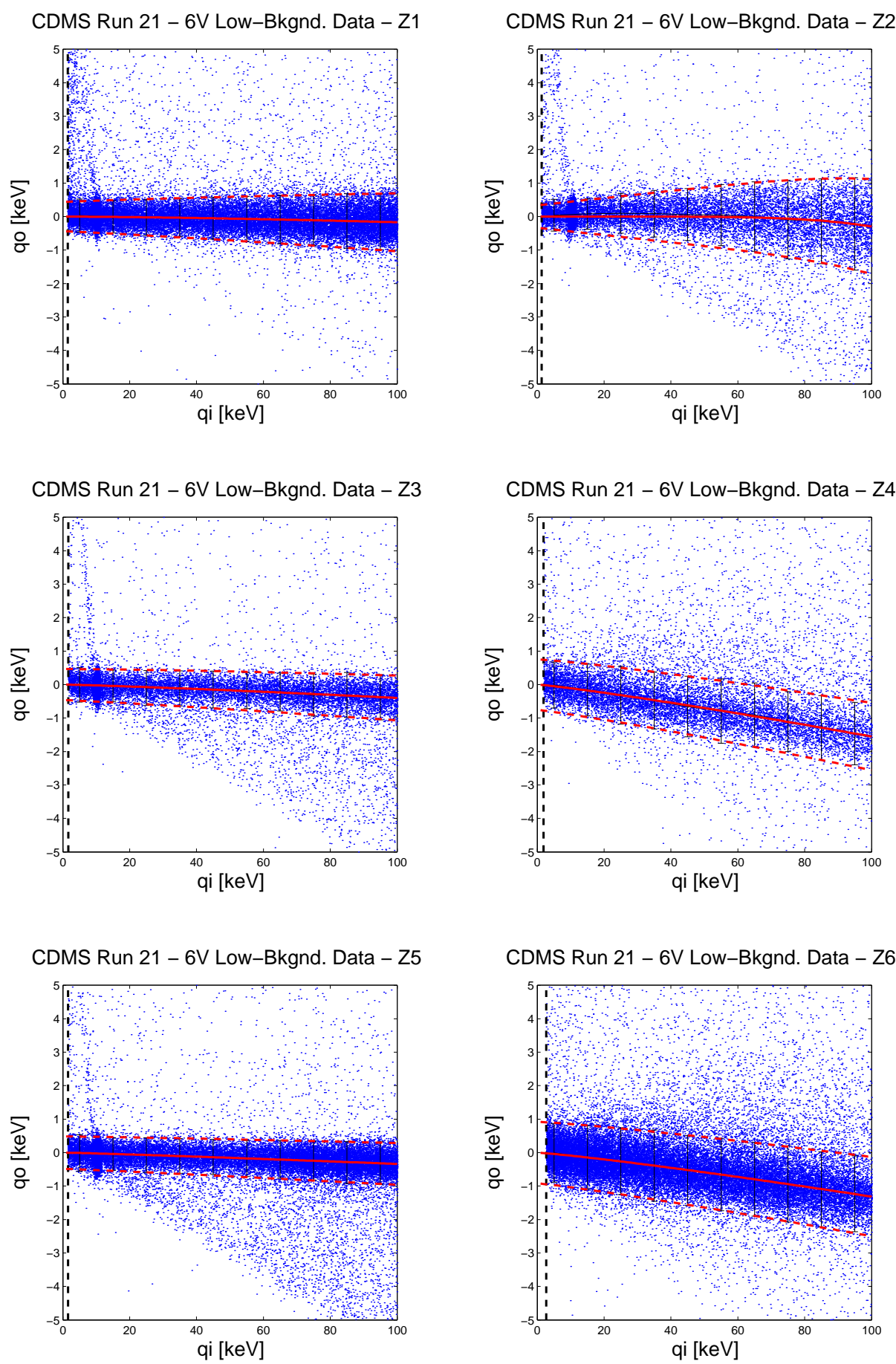

Figure A.6: Definition of the $6 \sigma$ q-inner noise threshold and the $2 \sigma$ q-outer noise bands $(3 \mathrm{~V})$. 


\section{A.3 Gamma Calibration}

The following section contains detailed plots of the electron-recoil band fitting routine described in Section 6.4. The graphs below show the bands and the Gaussian fits centered on the energy bins. The lines are the fits and the crosses are the fit points, where black $=$ mean and $\mathbf{r e d}=\operatorname{mean} \pm 3 \sigma(99.7 \% \mathrm{CL})$. At the top are listed "rnm" and "rnw", the residual norm of the fit for the mean and width fits respectively. 


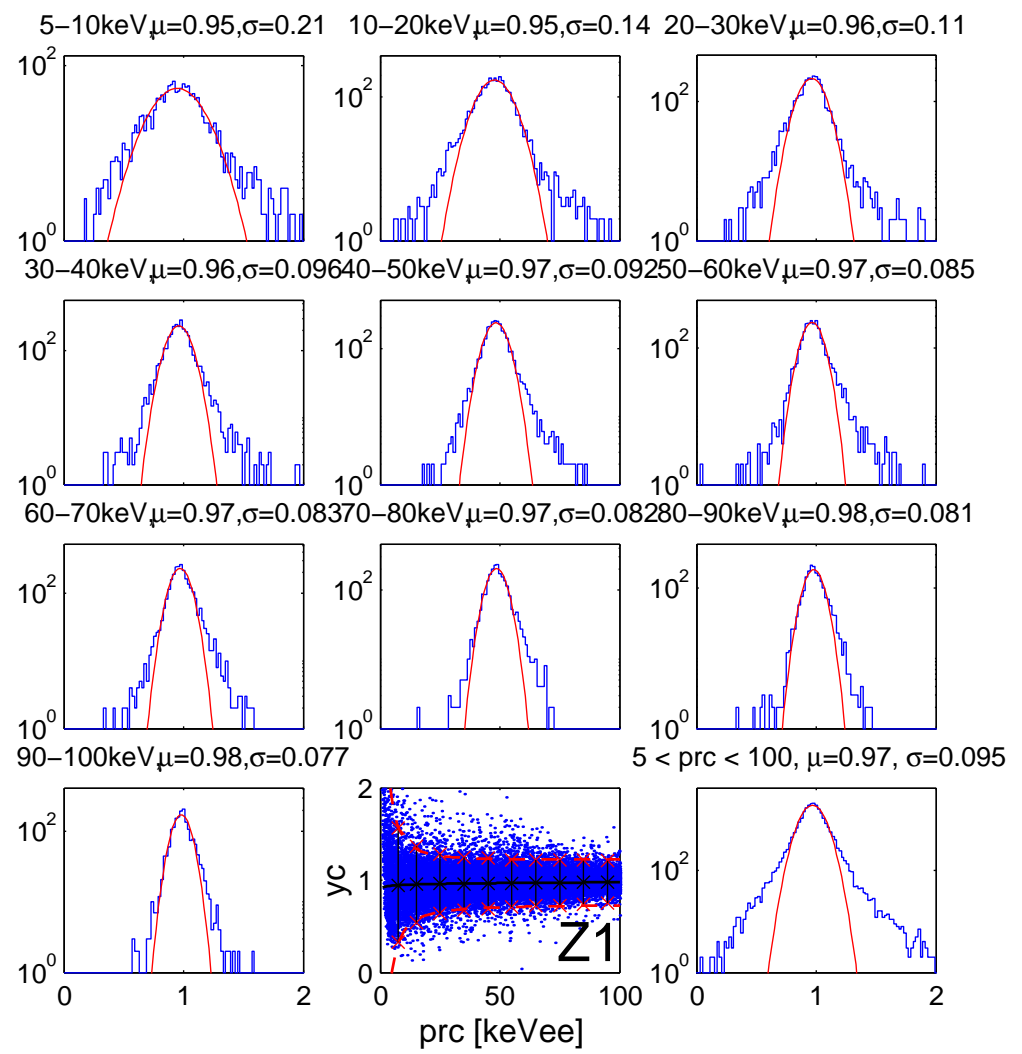

Figure A.7: Histograms of Yield in Slices of Energy in the Gamma Calibration Data, Detector Z1. In the histograms, the blue histogram is the data and the red line is the Gaussian fit to the peak. The title of each subplot lists the energy range, the mean, $\mu$ and standard deviation, $\sigma$, for the Gaussian fit. The bottom right plot shows a fit to the entire energy range, which is unused but shown for illustrative purposes. The bottom middle plot has vertical lines which represent the $\pm 3 \sigma$ levels from the individual bands overlaid on the datapoints from the calibration (blue). The results of the fit with respect to energy are also overlaid with the black line as the centroid, and the red lines as the $\pm 3 \sigma$ bands. 


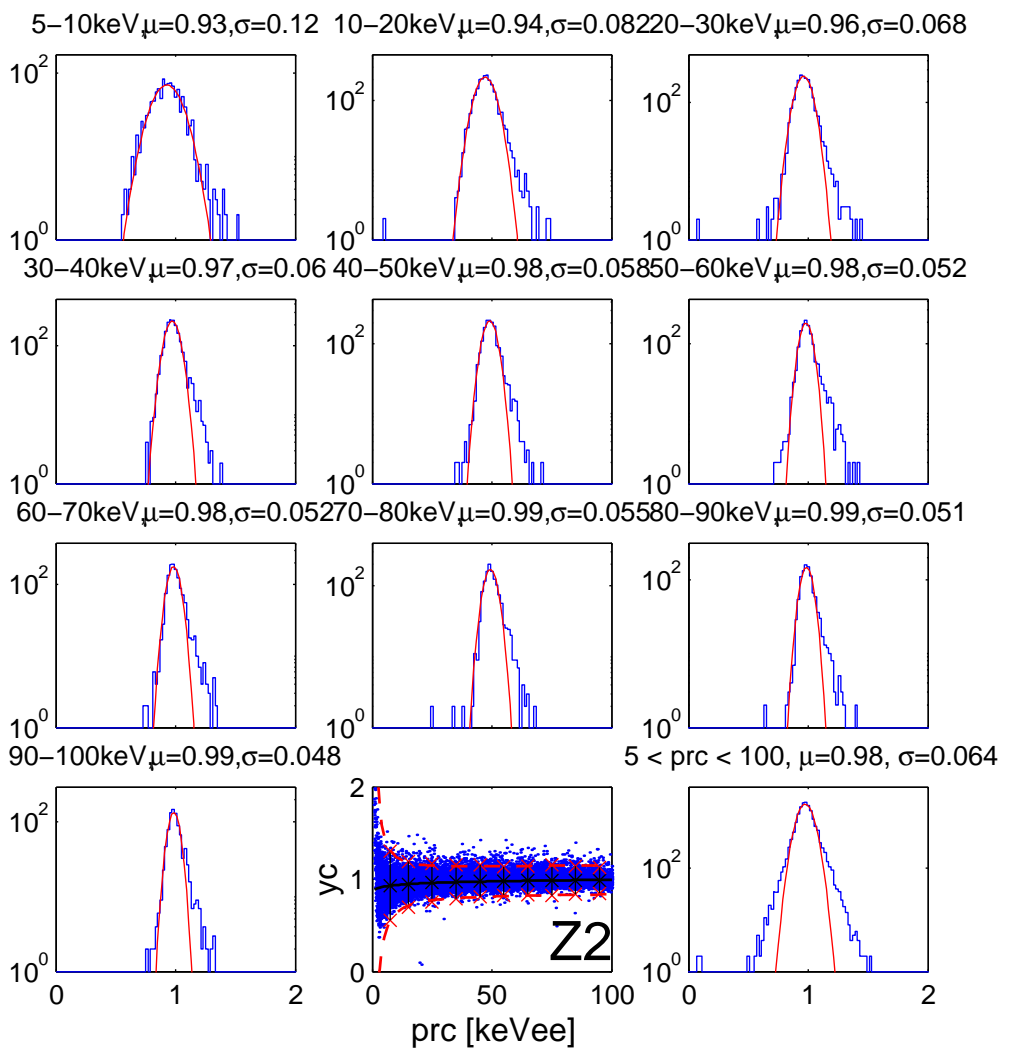

Figure A.8: Histograms of Yield in Slices of Energy in the Gamma Calibration Data, Detector Z2. In the histograms, the blue histogram is the data and the red line is the Gaussian fit to the peak. The title of each subplot lists the energy range, the mean, $\mu$ and standard deviation, $\sigma$, for the Gaussian fit. The bottom right plot shows a fit to the entire energy range, which is unused but shown for illustrative purposes. The bottom middle plot has vertical lines which represent the $\pm 3 \sigma$ levels from the individual bands overlaid on the datapoints from the calibration (blue). The results of the fit with respect to energy are also overlaid with the black line as the centroid, and the red lines as the $\pm 3 \sigma$ bands. 

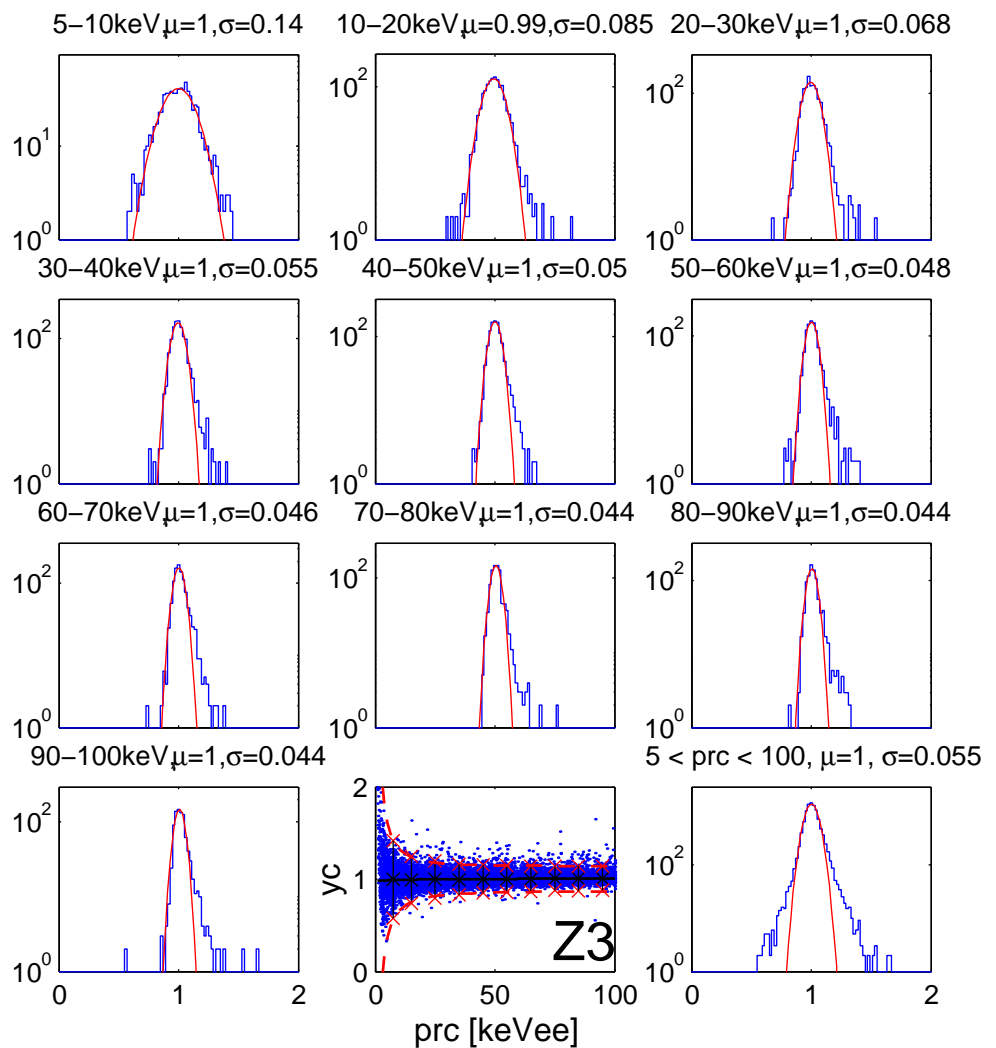

$\operatorname{prc}<100, \mu=1, \sigma=0.055$

Figure A.9: Histograms of Yield in Slices of Energy in the Gamma Calibration Data, Detector Z3. In the histograms, the blue histogram is the data and the red line is the Gaussian fit to the peak. The title of each subplot lists the energy range, the mean, $\mu$ and standard deviation, $\sigma$, for the Gaussian fit. The bottom right plot shows a fit to the entire energy range, which is unused but shown for illustrative purposes. The bottom middle plot has vertical lines which represent the $\pm 3 \sigma$ levels from the individual bands overlaid on the datapoints from the calibration (blue). The results of the fit with respect to energy are also overlaid with the black line as the centroid, and the red lines as the $\pm 3 \sigma$ bands. 

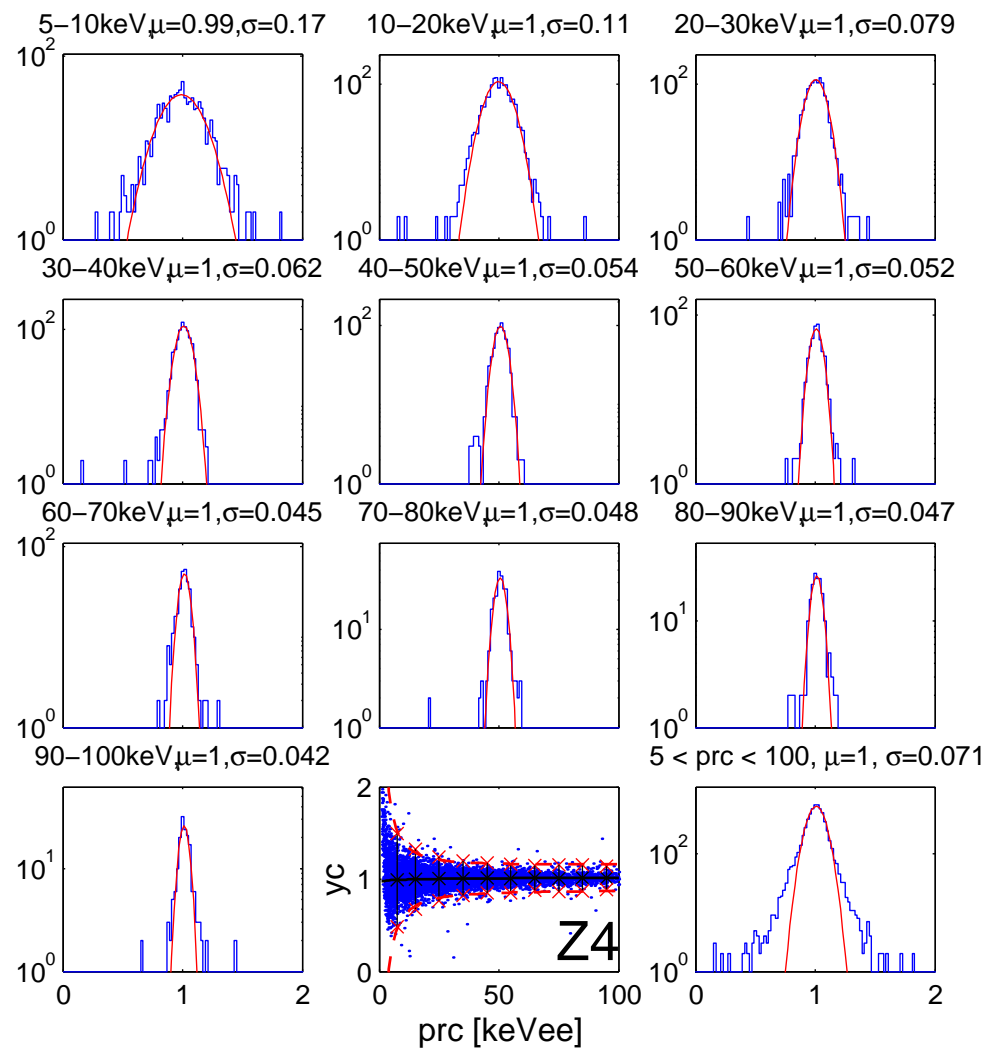

Figure A.10: Histograms of Yield in Slices of Energy in the Gamma Calibration Data, Detector Z4. In the histograms, the blue histogram is the data and the red line is the Gaussian fit to the peak. The title of each subplot lists the energy range, the mean, $\mu$ and standard deviation, $\sigma$, for the Gaussian fit. The bottom right plot shows a fit to the entire energy range, which is unused but shown for illustrative purposes. The bottom middle plot has vertical lines which represent the $\pm 3 \sigma$ levels from the individual bands overlaid on the datapoints from the calibration (blue). The results of the fit with respect to energy are also overlaid with the black line as the centroid, and the red lines as the $\pm 3 \sigma$ bands. 

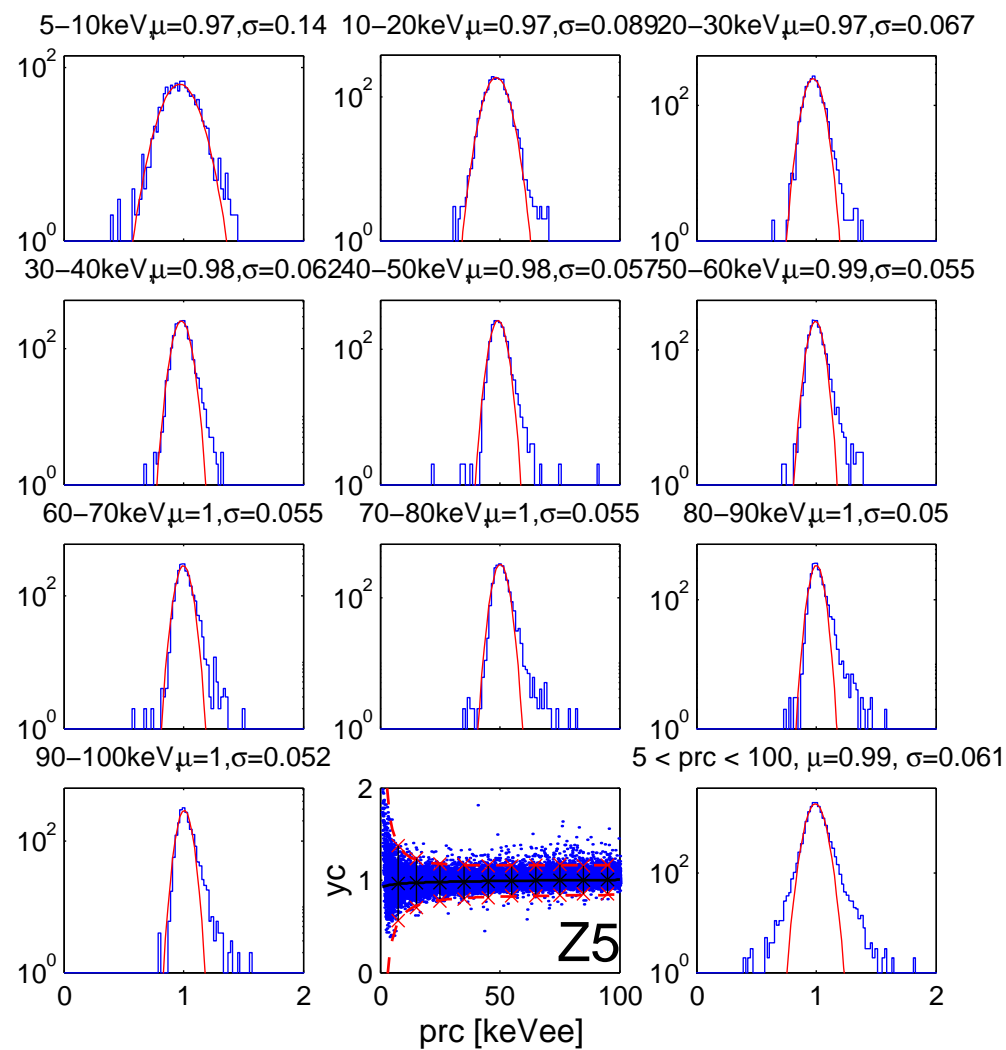

Figure A.11: Histograms of Yield in Slices of Energy in the Gamma Calibration Data, Detector Z5. In the histograms, the blue histogram is the data and the red line is the Gaussian fit to the peak. The title of each subplot lists the energy range, the mean, $\mu$ and standard deviation, $\sigma$, for the Gaussian fit. The bottom right plot shows a fit to the entire energy range, which is unused but shown for illustrative purposes. The bottom middle plot has vertical lines which represent the $\pm 3 \sigma$ levels from the individual bands overlaid on the datapoints from the calibration (blue). The results of the fit with respect to energy are also overlaid with the black line as the centroid, and the red lines as the $\pm 3 \sigma$ bands. 


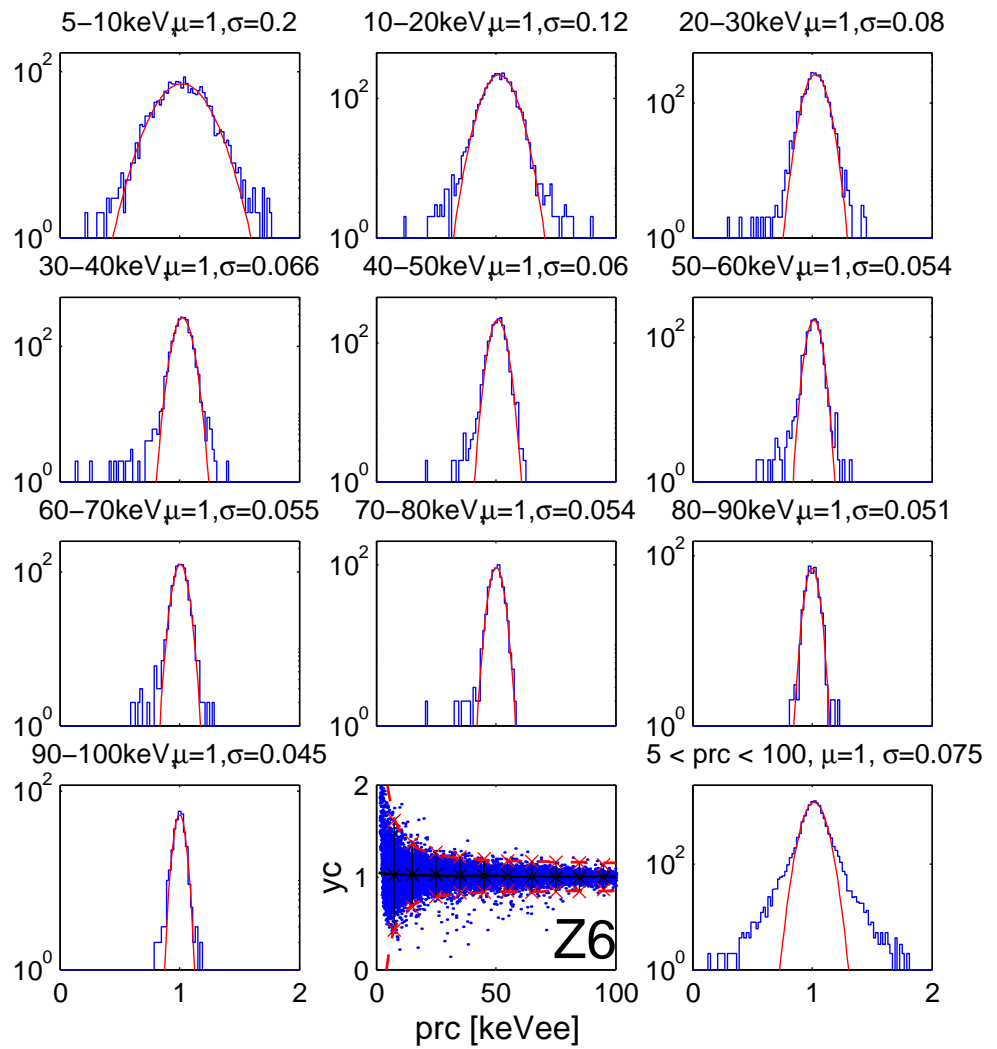

Figure A.12: Histograms of Yield in Slices of Energy in the Gamma Calibration Data, Detector Z6. In the histograms, the blue histogram is the data and the red line is the Gaussian fit to the peak. The title of each subplot lists the energy range, the mean, $\mu$ and standard deviation, $\sigma$, for the Gaussian fit. The bottom right plot shows a fit to the entire energy range, which is unused but shown for illustrative purposes. The bottom middle plot has vertical lines which represent the $\pm 3 \sigma$ levels from the individual bands overlaid on the datapoints from the calibration (blue). The results of the fit with respect to energy are also overlaid with the black line as the centroid, and the red lines as the $\pm 3 \sigma$ bands. 


\section{A.4 Neutron Calibration}

The following section contains detailed plots of the nuclear-recoil band fitting routine described in Section 6.5. 

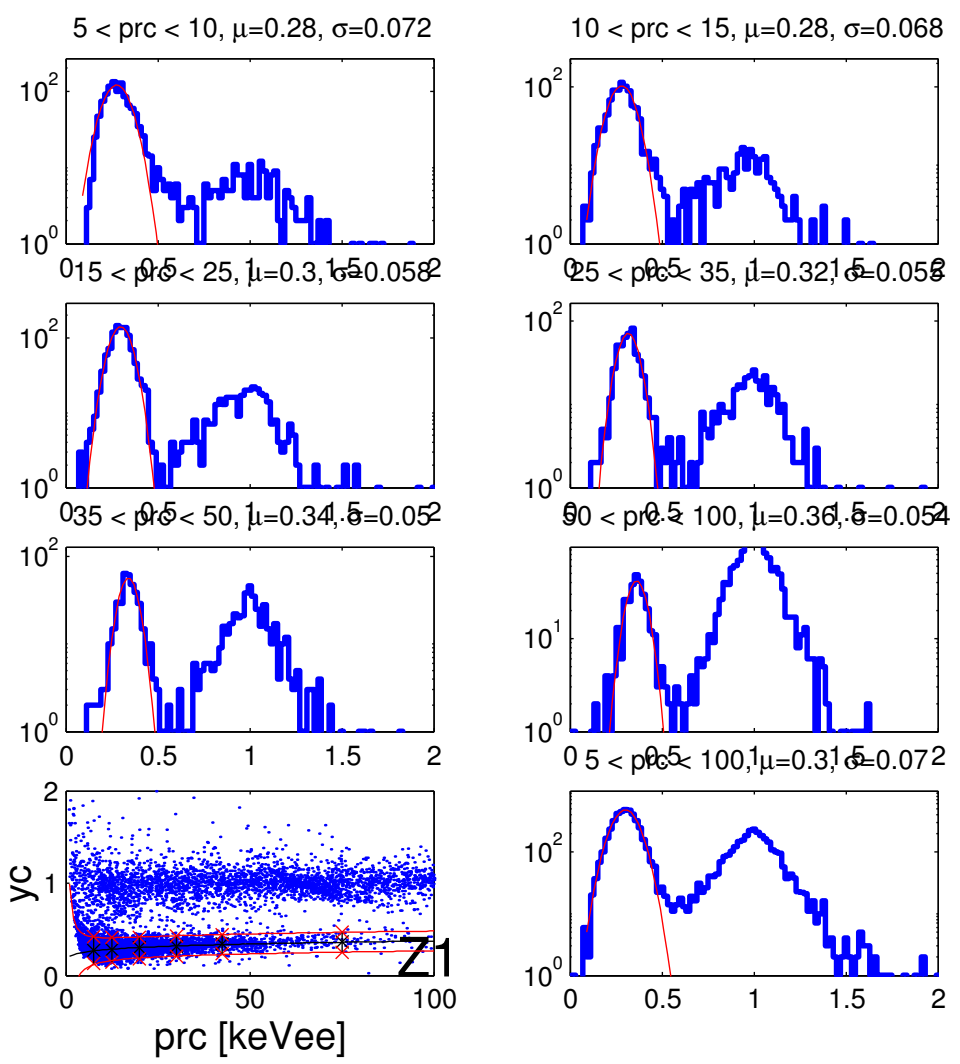

Figure A.13: Histograms of Yield in Slices of Energy in the Neutron Calibration Data, Detector Z1. In the histograms, the blue histogram is the data and the red line is the Gaussian fit to the peak. The title of each subplot lists the energy range, the mean, $\mu$ and standard deviation, $\sigma$, for the Gaussian fit. The bottom right plot shows a fit to the entire energy range, which is unused but shown for illustrative purposes. The bottom middle plot has vertical lines which represent the $\pm 3 \sigma$ levels from the individual bands overlaid on the datapoints from the calibration (blue). The results of the fit with respect to energy are also overlaid with the black line as the centroid, and the red lines as the $\pm 3 \sigma$ bands. 

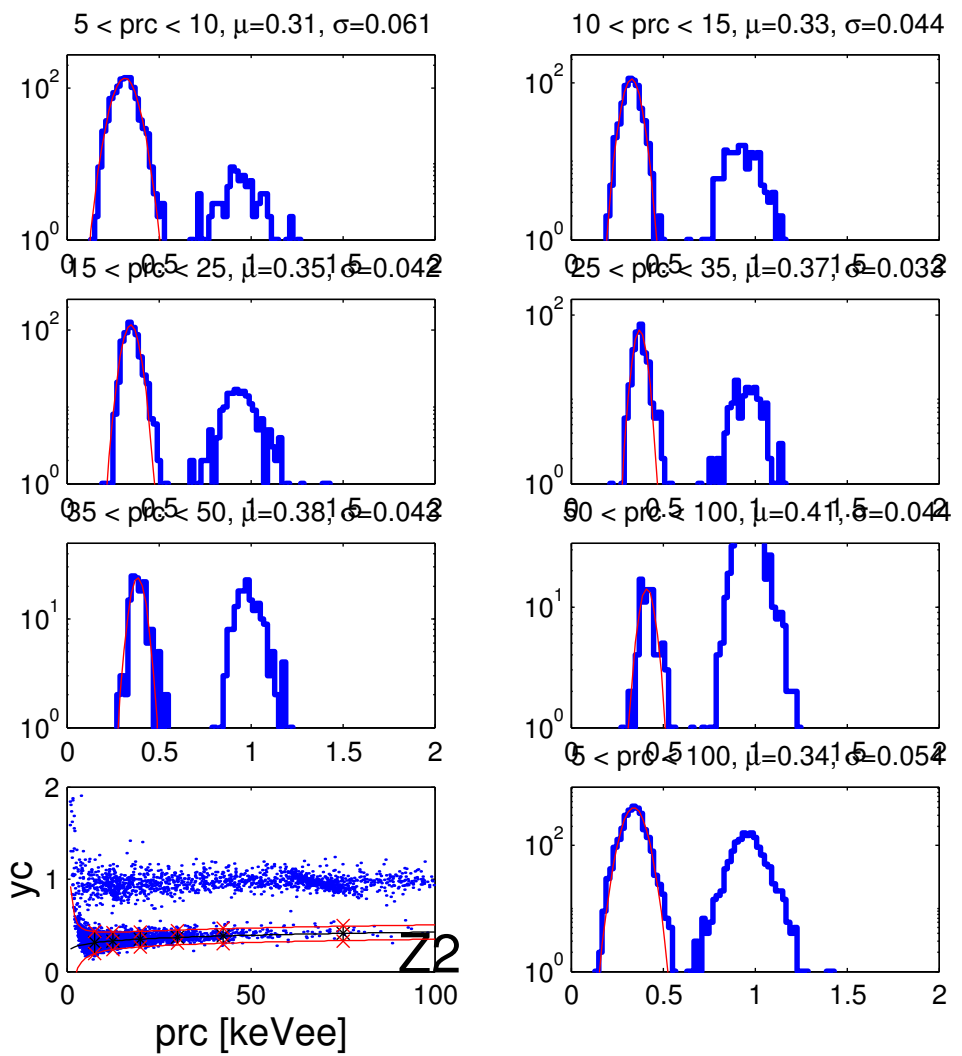

Figure A.14: Histograms of Yield in Slices of Energy in the Neutron Calibration Data, Detector Z2. In the histograms, the blue histogram is the data and the red line is the Gaussian fit to the peak. The title of each subplot lists the energy range, the mean, $\mu$ and standard deviation, $\sigma$, for the Gaussian fit. The bottom right plot shows a fit to the entire energy range, which is unused but shown for illustrative purposes. The bottom middle plot has vertical lines which represent the $\pm 3 \sigma$ levels from the individual bands overlaid on the datapoints from the calibration (blue). The results of the fit with respect to energy are also overlaid with the black line as the centroid, and the red lines as the $\pm 3 \sigma$ bands. 

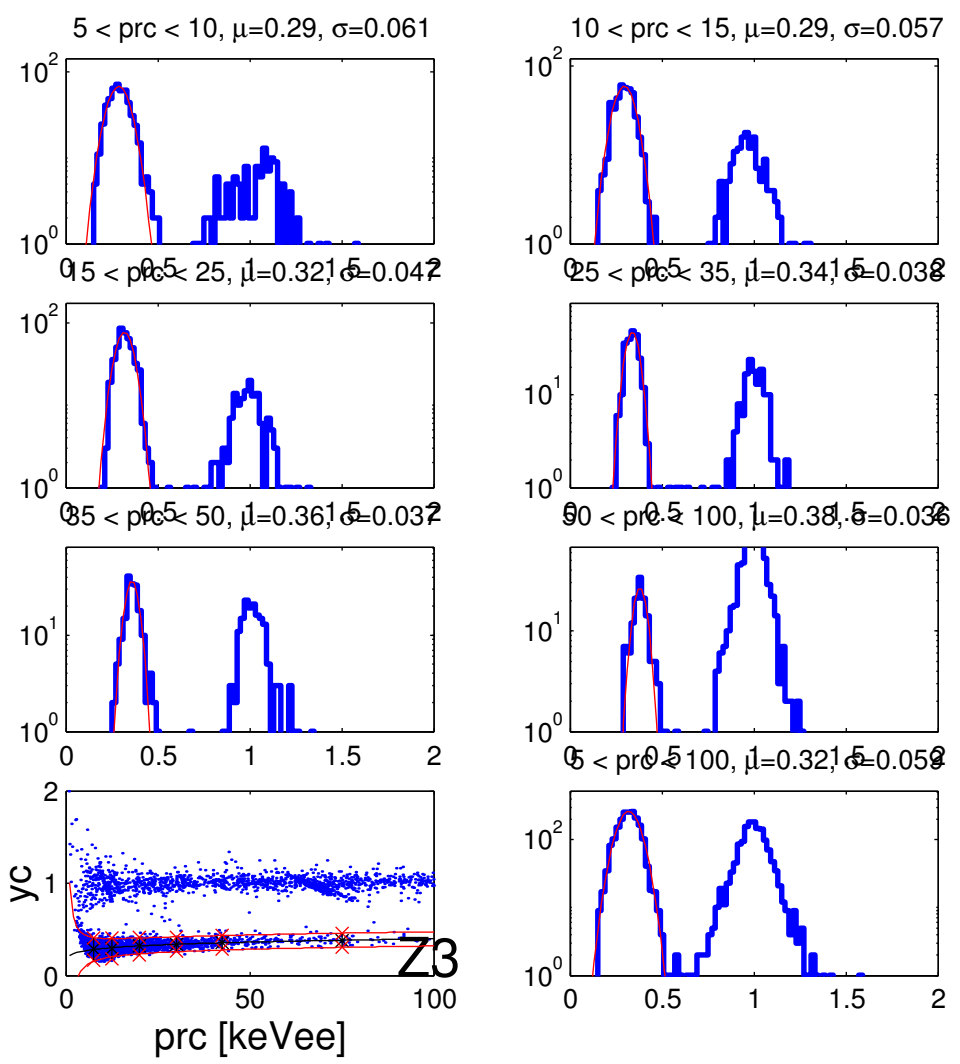

Figure A.15: Histograms of Yield in Slices of Energy in the Neutron Calibration Data, Detector Z3. In the histograms, the blue histogram is the data and the red line is the Gaussian fit to the peak. The title of each subplot lists the energy range, the mean, $\mu$ and standard deviation, $\sigma$, for the Gaussian fit. The bottom right plot shows a fit to the entire energy range, which is unused but shown for illustrative purposes. The bottom middle plot has vertical lines which represent the $\pm 3 \sigma$ levels from the individual bands overlaid on the datapoints from the calibration (blue). The results of the fit with respect to energy are also overlaid with the black line as the centroid, and the red lines as the $\pm 3 \sigma$ bands. 

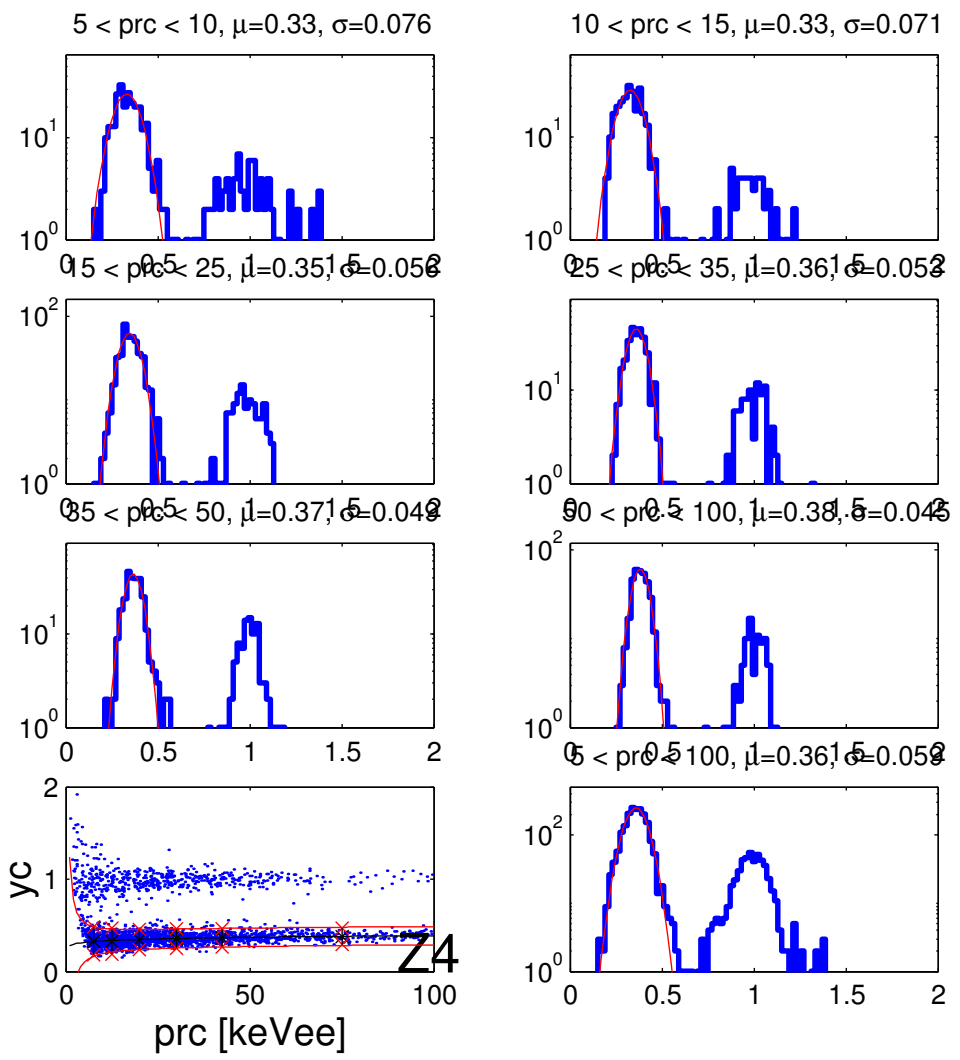

Figure A.16: Histograms of Yield in Slices of Energy in the Neutron Calibration Data, Detector Z4. In the histograms, the blue histogram is the data and the red line is the Gaussian fit to the peak. The title of each subplot lists the energy range, the mean, $\mu$ and standard deviation, $\sigma$, for the Gaussian fit. The bottom right plot shows a fit to the entire energy range, which is unused but shown for illustrative purposes. The bottom middle plot has vertical lines which represent the $\pm 3 \sigma$ levels from the individual bands overlaid on the datapoints from the calibration (blue). The results of the fit with respect to energy are also overlaid with the black line as the centroid, and the red lines as the $\pm 3 \sigma$ bands. 

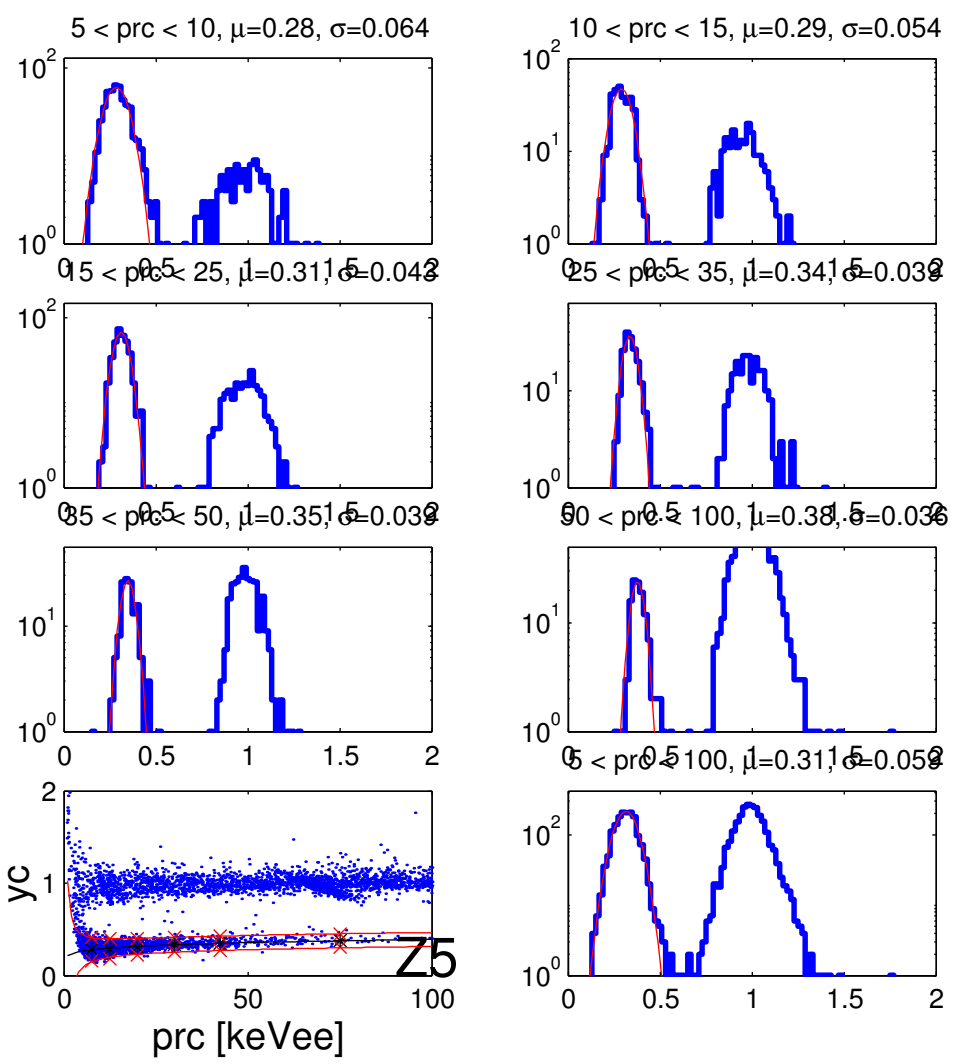

Figure A.17: Histograms of Yield in Slices of Energy in the Neutron Calibration Data, Detector Z5. In the histograms, the blue histogram is the data and the red line is the Gaussian fit to the peak. The title of each subplot lists the energy range, the mean, $\mu$ and standard deviation, $\sigma$, for the Gaussian fit. The bottom right plot shows a fit to the entire energy range, which is unused but shown for illustrative purposes. The bottom middle plot has vertical lines which represent the $\pm 3 \sigma$ levels from the individual bands overlaid on the datapoints from the calibration (blue). The results of the fit with respect to energy are also overlaid with the black line as the centroid, and the red lines as the $\pm 3 \sigma$ bands. 

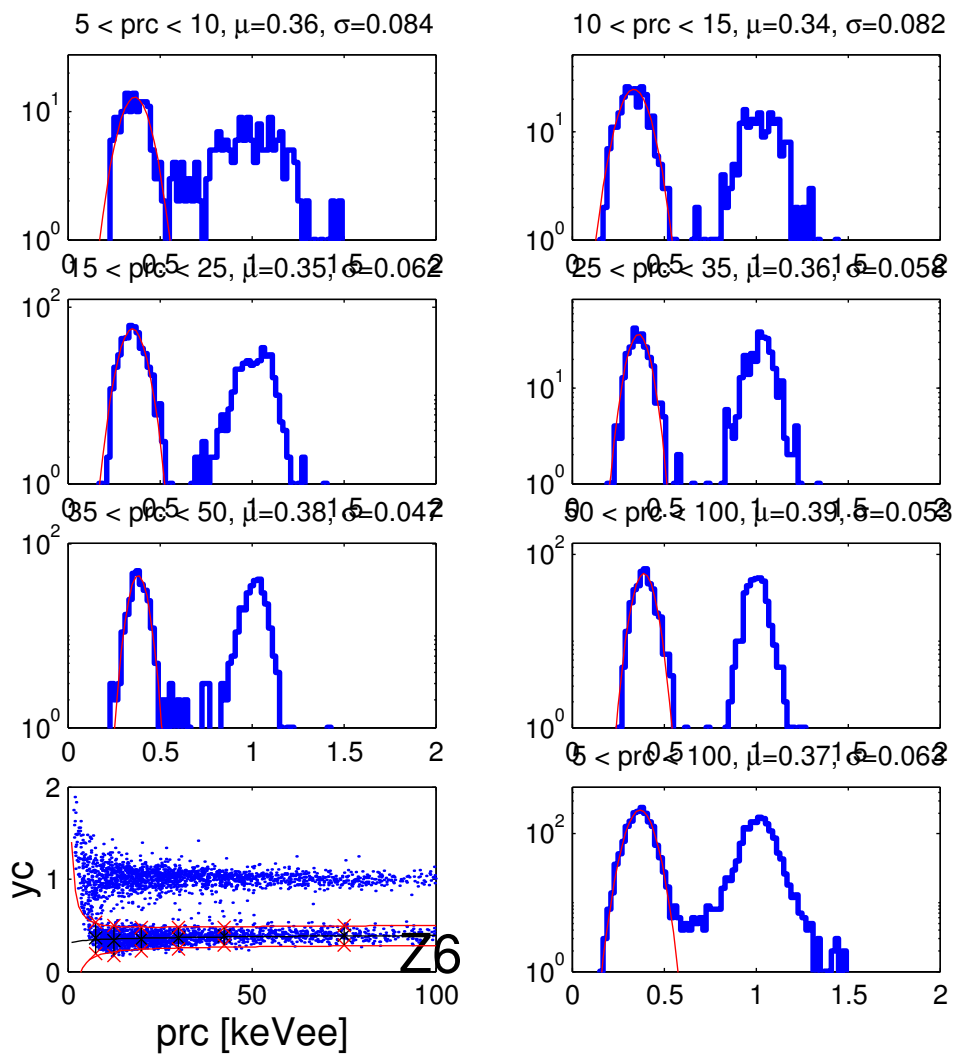

Figure A.18: Histograms of Yield in Slices of Energy in the Neutron Calibration Data, Detector Z6. In the histograms, the blue histogram is the data and the red line is the Gaussian fit to the peak. The title of each subplot lists the energy range, the mean, $\mu$ and standard deviation, $\sigma$, for the Gaussian fit. The bottom right plot shows a fit to the entire energy range, which is unused but shown for illustrative purposes. The bottom middle plot has vertical lines which represent the $\pm 3 \sigma$ levels from the individual bands overlaid on the datapoints from the calibration (blue). The results of the fit with respect to energy are also overlaid with the black line as the centroid, and the red lines as the $\pm 3 \sigma$ bands. 


\section{Bibliography}

D. Abrams et al. Exclusion limits on the wimp-nucleon cross section from the cryogenic dark matter search. Physical Review D, 66, 2002.

D.S. Akerib et al. New results from the cryogenic dark matter search experiment. Physical Review D, 68, October 2002.

M. Altmann et al. Results and plans of the CRESST dark matter search. June 2001. astro-ph/0106314.

E.A. Baltz and P. Gondolo. Implications of muon anomalous magnetic moment for supersymmetric dark matter. 2001. hep-ph/0102147.

P. Belli et al. DAMA Dark Matter Search. 1998. http://www.lngs.infn.it/lngs/report98/dama.pdf.

C.L. Bennett et al. First year Wilkinson Microwave Anisotropy Probe (WMAP) observations: Preliminary maps and basic results. 2003.

David Bennett. Magellanic cloud gravitational microlensing results: What do they mean? August 1998. astro-ph/9808121.

A. Benoit et al. Improved exclusion limits from the EDELWEISS WIMP search. June 2002. astro-ph/0206271.

R. Bernabei et al. New limits on WIMP search with large-mass low-radioactivity Na-I(Tl) set-up at gran sasso. Physical Review B, 389:757-766, December 1996.

R. Bernabei et al. Search for WIMP annual modulation signature: results from DAMA/NaI-3 and DAMA/NaI-4 and the global combined analysis. Physical Review B, 480:23-31, March 2000.

Paul Brink et al. Detector technical review. Internal note, August 2001. 
Paul Brink et al. G9 w tc distribution, before ion-implantation. Internal note, May 2001.

P.L. Brink et al. Determination of the tc distribution for 1000 transition edge sensors. In LTD-9 [36], pages 529-532.

B. Cabrera. Calculation of the phonon energy \& risetime corrections based on a 3d non-matrix approach. CDMS Run 21 ebook \#109, January 2002.

B. Cabrera et al. Prompt phonon signals from particle interactions in si crystals. In LTD-5 [35, pages 365-375.

R.M. Clarke. An Athermal Phonon Mediated Dark Matter Detector with Surface Event Discrimination. PhD thesis, Stanford University, May 1999.

R.M. Clarke et al. Enhanced ballistic phonon production for surface events in cryogenic silicon detector. Applied Physics Letters, 76(20):2958-2960, May 2000.

A.J. da Silva. Development of a Low Background Environment for the Cryogenic Dark Matter Search. PhD thesis, The University of British Columbia, April 1997.

D.D. Driscoll, Jr. Strange behaviour in q-outer. CDMS Run 21 ebook \#283, February 2003.

D.D. Driscoll, Jr. et al. A testing strategy for the mass production of CDMS II detectors. In LTD-9 [36], pages 533-536.

J. Ellis. Dark 2002 and beyond. astro-ph/0204059.

S.E. Esipov et al. Ballistic phonon emission from electron-hole droplets: Application to the nuclear recoil problem. In LTD-5 [35], pages 377-385.

G.J. Feldman and R. Cousins. Phys. Rev. D, (57):3873, 1998.

Sunil Ramanlal Golwala. Exclusion Limits on the WIMP-Nucleon Elastic-Scattering Cross Section from the Cryogenic Dark Matter Search. PhD thesis, The University of California at Berkeley, Fall 2000.

M.W. Goodman and E. Witten. Detectability of certain dark-matter candidates. Physical Review D, 31(12):3059-3063, June 1985.

N.W. Halverson et al. DASI first results: A measurement of the cosmic microwave background angular power spectrum. ASTROPHYSICAL JOURNAL, 568(38), May 2002. astro-ph/0104489. 
K.D. Irwin. Phonon-Mediated Particle Detection Using Superconducting Tungsten Transition-Edge Sensors. PhD thesis, Stanford University, February 1995.

Sharmila Kamat. Comparison of the external neutron Monte Carlo simulations to the 6V data. CDMS Internal Note, June 2003.

Sharmila Kamat. Estimating the limiting neutron background seen in run 21. CDMS Internal Note, January 2003.

Sharmila Kamat. Run 21 expected internal/external neutrons background for the calibrations. CDMS Internal Note, January 2003.

G.F. Knoll. Radiation Detection and Measurement. John Wiley and Sons, Inc., 1999.

A.T. Lee et al. A high spatial resolution analysis of the MAXIMA-1 cosmic microwave background anisotropy data. April 2001. astro-ph/0104459.

B.W. Lee and S. Weinberg. Cosmological lower bound on heavy-neutrino masses. Physical Review Letters, 39(4):165-168, July 1977.

J.D. Lewin and P.F. Smith. Review of mathematics, numerical factors, and corrections for dark matter experiments based on elastic nuclear recoil. Astroparticle Physics, 6:87-112, 1996.

Fifth International Workshop on Low Temperature Detectors, volume 93. Plenum Press, November 1993.

Ninth International Workshop on Low Temperature Detectors, volume 605. American Institute of Physics, July 2001.

P.N. Luke. Journal of Applied Physics, 64:6858, 1988.

Vuk Mandic. Fitting betas and neutrons. CDMS Run 21 ebook \#228, August 2002.

Vuk Mandic. Position dependence of double scatters. CDMS Run 21 ebook \#228, August 2002.

H.J. Maris and S. Tamura. Anharmonic decay and the propagation of phonons in an isotopically pure crystal at low temperatures: Application to dark-matter detection. Physical Review B, 47(2):727-739, January 1993.

Sae Woo Nam. Development of Phonon-Mediated Cryogenic Particle Detectors with Electron and Nuclear Recoil Discrimination. PhD thesis, Stanford University, December 1998. 
B. Neganov and V. Trofimov. USSR patent no. 1037771. 1985.

C.B. Netterfield et al. A measurement by BOOMERANG of multiple peaks in the angular power spectrum of the cosmic microwave background. Feb 2002. astro$\mathrm{ph} / 0104460$.

R.W. Ogburn. Parameterizing the box plot. CDMS Run 21 ebook \#291, April 2003.

K.A. Olive. Introduction to supersymmetry: Astrophysical and phenomenological constraints. November 1999. hep-ph/9911307, based on lectures delivered at the Les Houches Summer School, July 1999.

Particle Data Group. Review of Particle Physics, volume 3. Springer, 1998.

T.A. Perera. The Limiting Background in a Dark Matter Search at Shallow Depth. PhD thesis, Case Western Reserve University, January 2002.

S. Perlmutter et al. Measurements of $\omega$ and $\lambda$ from 42 high-redshift supernovae. The Astrophysical Journal, 517:565-586, June 1999.

C.P. Poole, Jr., editor. Handbook of Superconductivity. Academic Press, 2000.

W.H. Press, S.A. Teukolsky, W.T. Vetterling, and B.P. Flannery. Numerical Recipes in C: The Art of Scientific Computing. Cambridge University Press, 1992.

Angela Reisetter. A new veto cut using charge times and reprocessed data. CDMS Run 21 ebook \#296, June 2003.

V.C. Rubin and W.K. Ford, Jr. Rotation of the andromeda nebula from a spectroscopic survey of emission regions. The Astrophysical Journal, 159:379-403, February 1970.

T. Saab. Ibis curves as a useful sensor diagnostic. CDMSnote \#9911001, November 1999.

T. Saab. Measuring the abundance \& decay rate of the $10 \mathrm{kev}$ triplet. CDMS Run 21 ebook \#170, May 2002.

T. Saab. Search for Weakly Interacting Massive Particles with the Cryogenic Dark Matter Search Experiment. PhD thesis, Stanford University, August 2002.

V. Sanglard et al. The EDELWEISS experiment and dark matter direct detection. June 2003. astro-ph/0306233. 
Richard Schnee. Memo on calculating CDMS limits following Feldman and Cousins. CDMSnote 0105006, May 2001.

Richard Schnee. Choice of cuts for nearest-neighbor multiples. CDMS Run 21 ebook \#275, January 2003.

Table of Nuclides. http://www2.bnl.gov/ton/.

S. van den Bergh. The early history of dark matter. Publications of the Astronomical Society of the Pacific, June 1999. astro-ph/9904251.

R.P. Welty and J.M. Martinis. Two-stage integrated squid amplifier with series array output. IEEE Transactions on Applied Superconductivity, 3(1):2605-2608, March 1993.

S. Yellin. Physical Review D, 57:3873, 1998.

B.A. Young et al. Effect of implanted metal impurities on superconducting tungsten films. Journal of Applied Physics, 91(10), May 2002.

M.V. Zombeck. Handbook of Astronomy and Astrophysics, Second Edition. Cambridge University Press, 1990.

F. Zwicky. Helv. Phys. Acta, 6:110, 1933. 